\title{
Visible-Light-Mediated C-I Difluoroallylation with an $\alpha$-Aminoalkyl Radical as a Mediator
}

Fuyang Yue, Jianyang Dong, Yuxiu Liu and Qingmin Wang*

State Key Laboratory of Elemento-Organic Chemistry, Research Institute of Elemento-Organic

Chemistry, College of Chemistry, Frontiers Science Center for New Organic Matter, Nankai

University, Tianjin 300071, People's Republic of China

* Corresponding author E-mail: wangqm@nankai.edu.cn

\section{Supporting Information}

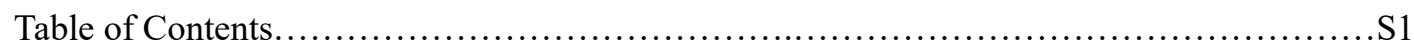

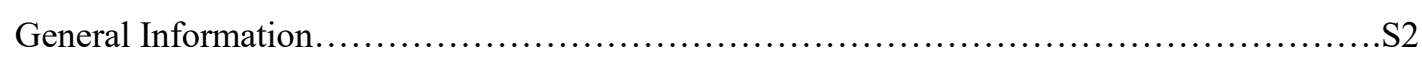

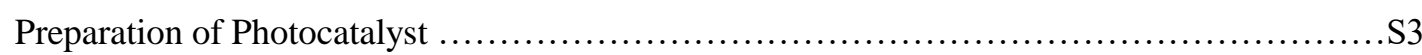

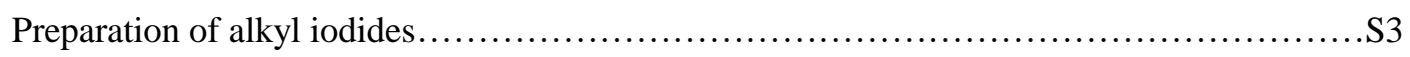

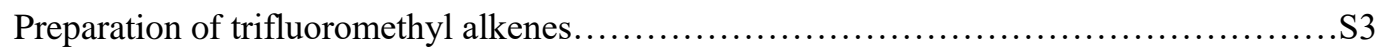

Investigation of the Key Reaction Parameters...........................................S5

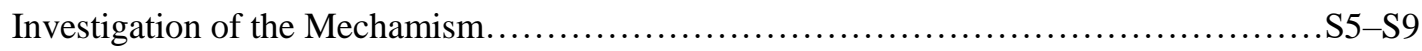

Experimental Procedures and Product Characterization...............................S9-S25

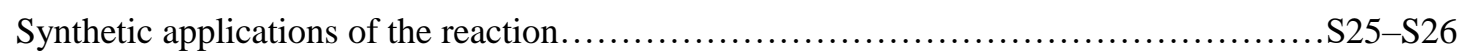

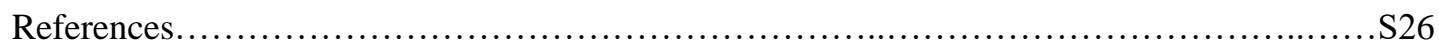

Copies of ${ }^{1} \mathrm{H}$ NMR and ${ }^{13} \mathrm{C}$ NMR spectra for new compounds........................S26-S79 


\section{General Information}

Reagents were purchased from commercial sources and were used as received. ${ }^{1} \mathrm{H}$ and ${ }^{13} \mathrm{C}$ Nuclear Magnetic Resonance (NMR) spectra were recorded on Bruker Avance 400 Ultrashield NMR spectrometers. Chemical shifts $(\delta)$ were given in parts per million (ppm) and were measured downfield from internal tetramethylsilane. High-resolution mass spectrometry (HRMS) data were obtained on an FTICR-MS instrument (Ionspec 7.0 T). The melting points were determined on an $\mathrm{X}-4$ microscope melting point apparatus and are uncorrected. Conversion was monitored by thin layer chromatography (TLC). Flash column chromatography was performed over silica gel $(100-200 \mathrm{mesh})$. Blue LED ( $36 \mathrm{~W}, \lambda_{\max }=470 \mathrm{~nm}$, UVA irradiance $=9.46 \mathrm{~mW} / \mathrm{m}^{2}$ ) purchased from JIADENG (LS) was used for blue light irradiation. A fan attached to the apparatus was used to maintain the reaction temperature at room temperature (about $30^{\circ} \mathrm{C}$ ).

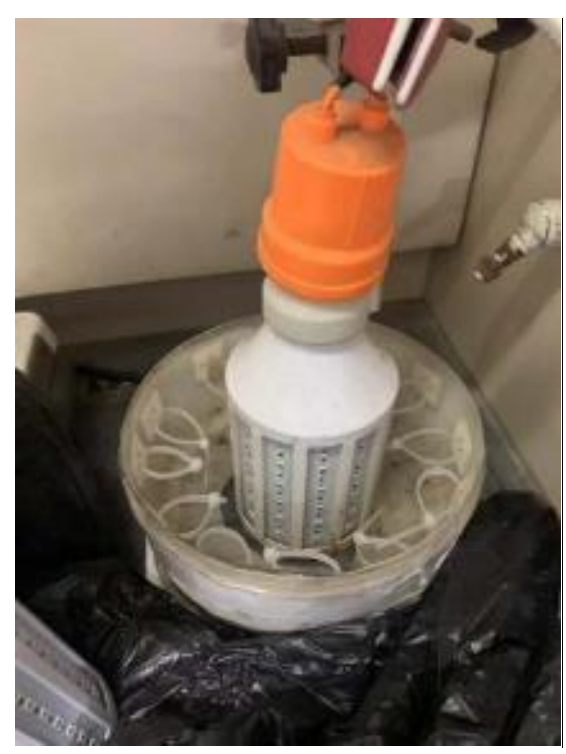

Figure S1 Photograph of the Photocatalytic reactor used for reactions conducted under blue LED irradiation. 


\section{Preparation of Photocatalyst}

The photocatalyst was synthesized according to literature report. ${ }^{[1]}$ The spectral data of the photocatalyst is consistent with the literature data. The other photocatalysts (Eosin Y, 4CzIPN, $\left.\left[\mathrm{Ru}(\mathrm{bpy})_{3}\right] \mathrm{Cl}_{2} \cdot 6 \mathrm{H}_{2} \mathrm{O}, \mathrm{Ru}(\mathrm{bpy})_{3}\left(\mathrm{PF}_{6}\right)_{2}, \operatorname{Ir}(\mathrm{ppy})_{3}\right)$ are commercially available.

\section{Conversion of alcohols to alkyl iodides.}

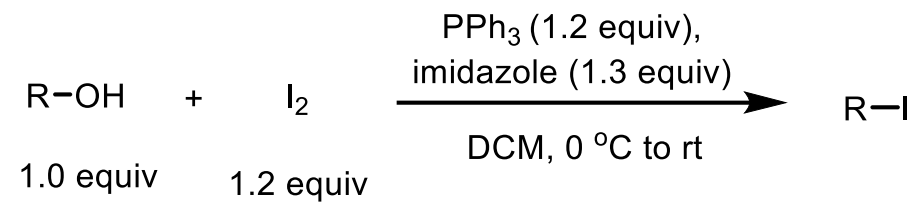

The alkyl iodides were synthesized according to literature report. ${ }^{2}$ A $200 \mathrm{~mL}$ oven-dried round-bottom flask equipped with a stirbar and rubber septum is cooled under a stream of nitrogen. The flask was opened to air, and triphenylphosphine (1.2 equiv), imidazole (1.3 equiv), and DCM (non-anhydrous) were added under air. The rubber septum was replaced, and the flask was purged with nitrogen for ca. $5 \mathrm{~min}$. The flask was then placed in an ice-bath. After $5 \mathrm{~min}$ of stirring, the rubber septum was partly removed and iodine (1.2 equiv) was added under a stream of nitrogen. The rubber septum was replaced and the reaction was stirred for another $15 \mathrm{~min}$. The alcohol was then added. For solid alcohols, the rubber septum was partly removed, the alcohol was added under a stream of nitrogen, and the rubber septum was returned. For liquid alcohols, the alcohol was added via syringe through the rubber septum. The spectral data of the alkyl iodides are consistent with the literature data. ${ }^{2}$

\section{General procedure for the preparation of trifluoromethyl alkenes}

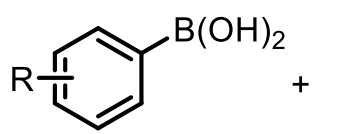

1.0 equiv<smiles>C=C(Br)C(F)(F)F</smiles>

1.5 equiv

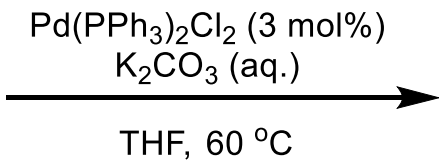<smiles></smiles>

According to literature reports. ${ }^{3}$ A Schlenk tube equipped with stir bar, arylboronic acid (1.0 equiv, $3 \mathrm{mmol})$ and $\mathrm{Pd}\left(\mathrm{PPh}_{3}\right)_{2} \mathrm{Cl}_{2}(3 \mathrm{~mol} \%, 0.09 \mathrm{mmol}, 63.2 \mathrm{mg})$ were added. The vessel was evacuated and filled with argon (three times), and then aqueous $\mathrm{K}_{2} \mathrm{CO}_{3}(2.0 \mathrm{M}, 6 \mathrm{~mL})$ and $\mathrm{THF}(9 \mathrm{~mL})$ were added. After addition of 2-bromo-3,3,3-trifluoro-1-propene (1.5 equiv, $4.5 \mathrm{mmol}, 0.47 \mathrm{~mL}$ ), the solution was stirred at $60{ }^{\circ} \mathrm{C}$ with heating mantle for 12 hours (TLC tracking detection). The solvent was removed under reduced pressure and the residue was purified by column chromatography to afford the corresponding trifluoromethyl alkene (PE/EA). The spectral data is consistent with the literature data. ${ }^{3}$ 


\section{Investigation of the Key Reaction Parameters}

\section{Table S1: Screening of different solvents ${ }^{\mathrm{a}}$}<smiles>C=C(c1ccc(OCc2ccccc2)cc1)C(F)(F)F</smiles>

$1,1.0$ equiv<smiles>IC1CCCCC1</smiles>

2, 2.0 equiv
$1 \mathrm{~mol} \% \operatorname{lr}\left[\mathrm{dF}\left(\mathrm{CF}_{3}\right) \mathrm{ppy}_{2}\right]_{2}(\mathrm{dtbbpy}) \mathrm{PF}_{6}$

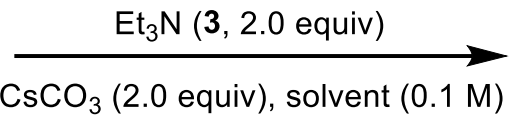

r.t. Ar, $24 \mathrm{~h}$

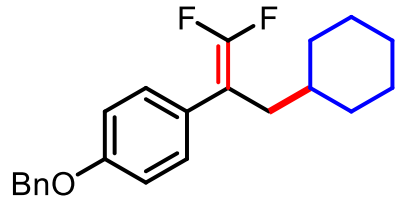

4

\begin{tabular}{lll}
\hline entry & solvent & yield $(\%)^{\mathrm{b}}$ \\
\hline 1 & $\mathrm{CH}_{3} \mathrm{CN}$ & 50 \\
2 & DMSO & 20 \\
3 & acetone & trace \\
4 & $\mathrm{DCM}$ & 50 \\
5 & $\mathrm{DMF}$ & 11 \\
6 & $\mathrm{DMA}$ & 17 \\
7 & $\mathrm{CH}_{3} \mathrm{OH}$ & trace \\
8 & $\mathrm{EtOH}$ & 22 \\
9 & $\mathrm{EA}$ & trace \\
10 & $\mathrm{HCCl}$ & $\mathrm{NR}$ \\
11 & $\mathrm{DCE}$ & 87 \\
12 & $\mathrm{THF}$ & $\mathrm{NR}$ \\
13 & $1,4-$ dioxane & $\mathrm{NR}$ \\
\hline
\end{tabular}

${ }^{\mathrm{a}}$ General conditions: $1(0.2 \mathrm{mmol}), 2(0.4 \mathrm{mmol}), \operatorname{Ir}\left[\mathrm{dF}_{(}\left(\mathrm{CF}_{3}\right) \text { ppy }\right]_{2}(\mathrm{dtbbpy}) \mathrm{PF}_{6}(0.002 \mathrm{mmol})$, base $(0.4 \mathrm{mmol})$, triethylamine $(\mathbf{3}, 0.4 \mathrm{mmol})$, solvent $(2 \mathrm{~mL}), \mathrm{rt}$, Ar atmosphere, $24 \mathrm{~h}$. ${ }^{\text {bYields were }}$ determined by ${ }^{19} \mathrm{~F}$ NMR spectroscopy using fluorobenzene as an internal standard. $\mathrm{NR}=$ no reaction.

Table S2: Screening of different base ${ }^{a}$<smiles>C=C(c1ccc(OCc2ccccc2)cc1)C(F)(F)F</smiles>

$1,1.0$ equiv<smiles>IC1CCCCC1</smiles>

$1 \mathrm{~mol} \% \operatorname{Ir}\left[\mathrm{dF}\left(\mathrm{CF}_{3}\right) \mathrm{ppy}\right]_{2}(\mathrm{dtbbpy}) \mathrm{PF}_{6}$

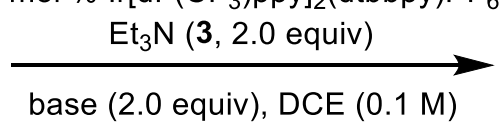

r.t. Ar, $24 \mathrm{~h}$

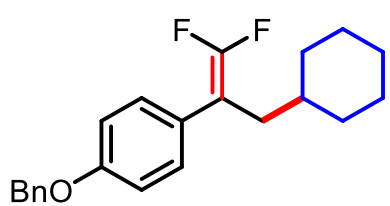

4

\begin{tabular}{lll}
\hline entry & base & yield $(\%)^{\mathrm{b}}$ \\
\hline 1 & $\mathrm{Na}_{2} \mathrm{CO}_{3}$ & 20 \\
2 & $\mathrm{NaHCO}_{3}$ & 13 \\
3 & $\mathrm{~K}_{2} \mathrm{CO}_{3}$ & 35 \\
4 & $\mathrm{~K}_{3} \mathrm{PO}_{4}$ & $96\left(90^{\mathrm{c}}\right)$ \\
5 & $\mathrm{~K}_{2} \mathrm{HPO}_{4}$ & 70 \\
6 & $\mathrm{Na}_{2} \mathrm{OAc}$ & 36 \\
7 & $\mathrm{CsOAc}$ & 50 \\
8 & $\mathrm{DIPEA}$ & 64 \\
9 & $\mathrm{Et}_{3} \mathrm{~N}$ & 81 \\
\hline
\end{tabular}

${ }^{\mathrm{a}}$ General conditions: $1(0.2 \mathrm{mmol}), \mathbf{2}(0.4 \mathrm{mmol}), \operatorname{Ir}\left[\mathrm{dF}_{(}\left(\mathrm{CF}_{3}\right) \mathrm{ppy}\right]_{2}(\mathrm{dtbbpy}) \mathrm{PF}_{6}(0.002 \mathrm{mmol})$, base 
$(0.4 \mathrm{mmol})$, triethylamine $(\mathbf{3}, 0.4 \mathrm{mmol})$, solvent $(2 \mathrm{~mL}), \mathrm{rt}$, Ar atmosphere, $24 \mathrm{~h} .{ }^{\text {bY }}$ ields were determined by ${ }^{19} \mathrm{~F}$ NMR spectroscopy using fluorobenzene as an internal standard. $\mathrm{NR}=$ no reaction. 'Isolated yield.

Table S3: Screening of photocatalysts ${ }^{\mathrm{a}}$<smiles>C=C(c1ccc(OC(C)(F)F)cc1)C(F)(F)F</smiles>

$1,1.0$ equiv<smiles>IC1CCCCC1</smiles>

2, 2.0 equiv

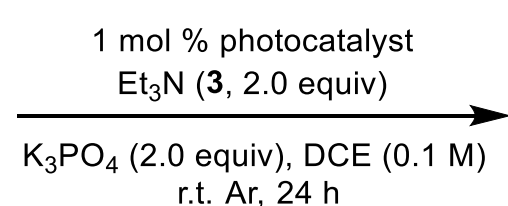

r.t. Ar, $24 \mathrm{~h}$<smiles>FC(F)=C(CC1CCCCC1)c1ccc(OCc2ccccc2)cc1</smiles>

4

\begin{tabular}{lll}
\hline entry & photocatalyst & yield $(\%)^{\mathrm{b}}$ \\
\hline 1 & {$\left[\mathrm{Ir}(\mathrm{dtbbpy})(\mathrm{ppy})_{2}\right]\left[\mathrm{PF}_{6}\right]$} & trace \\
2 & {$\left[\mathrm{Ru}(\mathrm{bpy})_{3}\right]\left(\mathrm{PF}_{6}\right)_{2}$} & $\mathrm{NR}$ \\
3 & {$\left[\mathrm{Ru}(\mathrm{bpy})_{3}\right] \mathrm{Cl}_{2} 6 \mathrm{H}_{2} \mathrm{O}$} & $\mathrm{NR}$ \\
$4^{\mathrm{c}}$ & $4 \mathrm{CzIPN}$ & 75 \\
$5^{\mathrm{c}}$ & Eosin-Y & $\mathrm{NR}$ \\
6 & $\operatorname{Ir}\left[\mathrm{dF}\left(\mathrm{CF}_{3}\right) \mathrm{ppy}_{2}(\mathrm{dtbbpy}) \mathrm{PF}_{6}\right.$ & 96
\end{tabular}

${ }^{\mathrm{a}}$ General conditions: $1(0.2 \mathrm{mmol}), 2(0.4 \mathrm{mmol}), \operatorname{Ir}\left[\mathrm{dF}_{(}\left(\mathrm{CF}_{3}\right) \text { ppy }\right]_{2}(\mathrm{dtbbpy}) \mathrm{PF}_{6}(0.002 \mathrm{mmol})$, base $(0.4 \mathrm{mmol})$, triethylamine $(\mathbf{3}, 0.4 \mathrm{mmol})$, solvent $(2 \mathrm{~mL}), \mathrm{rt}$, Ar atmosphere, $24 \mathrm{~h}$. ${ }^{\text {bYields were }}$ determined by ${ }^{19} \mathrm{~F}$ NMR spectroscopy using fluorobenzene as an internal standard. $\mathrm{NR}=$ no

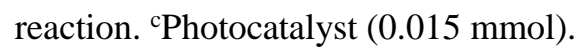

\section{Investigation of the Mechamism}

\subsection{Control experiments}

\section{Table S4}

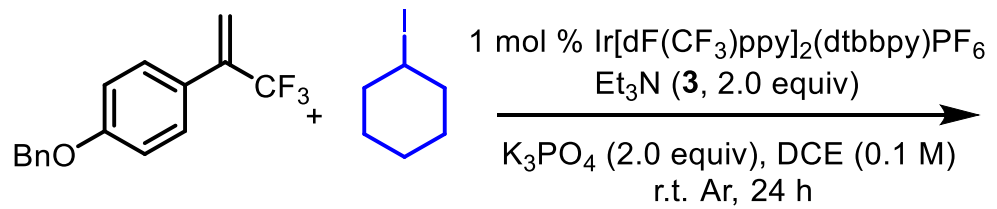

$1,1.0$ equiv

2, 2.0 equiv<smiles>FC(F)=C(CC1CCCCC1)c1ccc(OCc2ccccc2)cc1</smiles>

\begin{tabular}{lll}
\hline entry & control conditions & yield $(\%)^{\mathrm{b}}$ \\
\hline 1 & w/o photocatalyst & NR \\
2 & w/o light & NR \\
3 & w/o 3 & NR \\
4 & w/o base & 30 \\
5 & Under air & 56 \\
6 & standard conditions, w/all & 96 \\
\hline
\end{tabular}


${ }^{\mathrm{a}}$ General conditions: $1(0.2 \mathrm{mmol}), \mathbf{2}(0.4 \mathrm{mmol}), \operatorname{Ir}\left[\mathrm{dF}_{(}\left(\mathrm{CF}_{3}\right) \mathrm{ppy}_{2}(\mathrm{dtbbpy}) \mathrm{PF}_{6}(0.002 \mathrm{mmol})\right.$, base $(0.4 \mathrm{mmol})$, triethylamine $(\mathbf{3}, 0.4 \mathrm{mmol})$, solvent $(2 \mathrm{~mL})$, rt, Ar atmosphere, $24 \mathrm{~h}$. ${ }^{\text {bY }}$ ields were determined by ${ }^{19} \mathrm{~F}$ NMR spectroscopy using fluorobenzene as an internal standard. $\mathrm{NR}=$ no

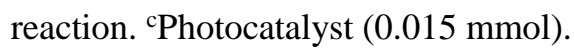

\subsection{TEMPO, BHT and 1,1-diphenylethylene were used as radical scavengers}<smiles>C=C(c1ccc(Br)cc1)C(F)(F)F</smiles>

$1,1.0$ equiv<smiles>IC1CCCCC1</smiles>

2, 2.0 equiv

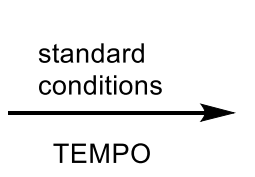

TEMPO<smiles>FC(F)=C(CC1CCCCC1)c1ccc(OCc2ccccc2)cc1</smiles>

4, $0 \%$

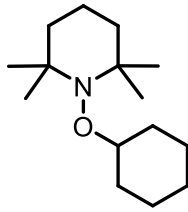

42, Detected by HRMS $[\mathrm{M}+\mathrm{H}]^{+}$Calc. 240.2322 Found: 240.2318<smiles>C=C(c1ccc(OCc2ccccc2)cc1)C(F)(F)F</smiles>

1, 1.0 equiv<smiles>IC1CCCCC1</smiles>

2, 2.0 equiv

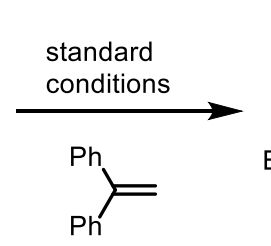<smiles>FC(F)=C(CC1CCCCC1)c1ccc(OCc2ccccc2)cc1</smiles>

4, $20 \%$<smiles>C(=C(c1ccccc1)C1CCCCC1)c1ccccc1</smiles>

43, Detected by HRMS $[\mathrm{M}+\mathrm{H}]^{+}$Calc. 263.1794 Found: 263.1791

\section{Scheme S1}

To a $10 \mathrm{~mL}$ glass vial was added $\operatorname{Ir}\left[\mathrm{dF}\left(\mathrm{CF}_{3}\right) \text { ppy) }\right]_{2}(\mathrm{dtbbpy}) \mathrm{PF}_{6}(2.24 \mathrm{mg}, 0.002 \mathrm{mmol}, 1 \mathrm{~mol} \%), \mathbf{1}$ (0.2 mmol, 1.0 equiv), 2 (0.4 mmol, 2.0 equiv), TEMPO (0.4 mmol, 2.0 equiv) or 1,1-diphenylethylene ( $0.4 \mathrm{mmol}, 2.0$ equiv), base $(0.4 \mathrm{mmol})$, triethylamine $(\mathbf{3}, 0.4 \mathrm{mmol})$, solvent $(2 \mathrm{~mL}), \mathrm{rt}, \mathrm{Ar}$ atmosphere. The mixture was then stirred rapidly and irradiated with a $36 \mathrm{~W}$ Blue LED (approximately $2 \mathrm{~cm}$ away from the light source) at room temperature for $24 \mathrm{~h}$. The corresponding alkylated product 4 was observed based on ${ }^{19} \mathrm{~F}$ NMR analysis, and instead the corresponding product of radical trapping, 1-isopropoxy-2,2,6,6-tetramethylpiperidine (42), (2-cyclohexylethene-1,1-diyl)dibenzene (43), was detected by HR-MS (positive mode ESI). 

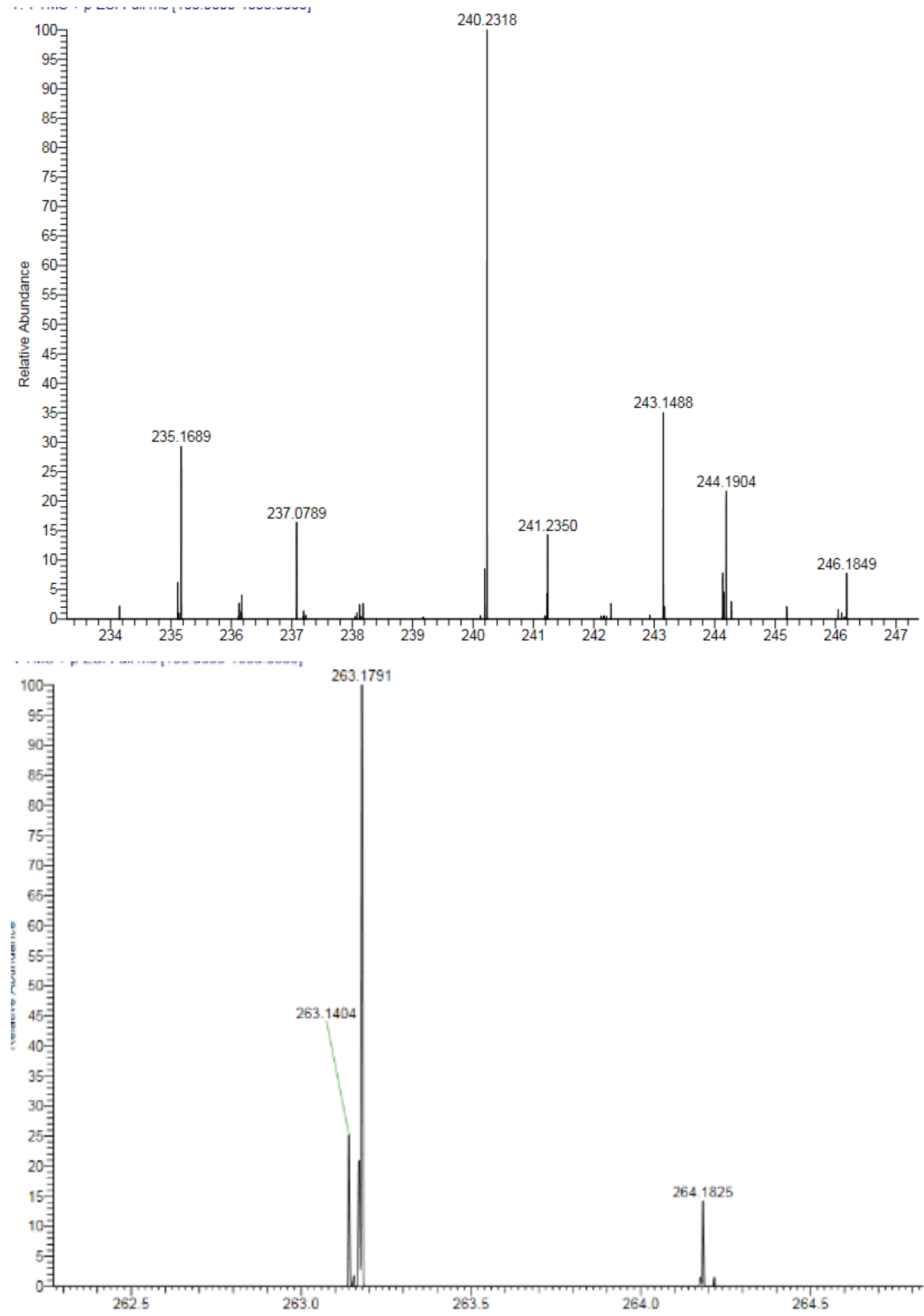

Figure S3 HR-ESI mass spectra of 1-isopropoxy-2,2,6,6-tetramethylpiperidine (36)

\subsection{Light/dark experiment}


<smiles>C=C(c1ccc(OC(F)(F)Br)cc1)C(F)(F)F</smiles>

$1,1.0$ equiv<smiles>IC1CCCCC1</smiles>

2, 2.0 equiv

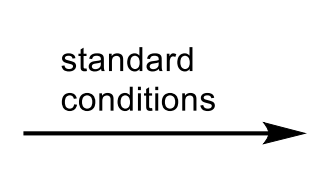<smiles>FC(F)=C(CC1CCCCC1)c1ccc(OCc2ccccc2)cc1</smiles>

4,

To a $10 \mathrm{~mL}$ glass vials were charged with $\operatorname{Ir}\left[\mathrm{dF}\left(\mathrm{CF}_{3}\right) \text { ppy) }\right]_{2}(\mathrm{dtbbpy}) \mathrm{PF}_{6}(2.24 \mathrm{mg}, 0.002 \mathrm{mmol}, 1$ mol \%), 1 ( $0.2 \mathrm{mmol}, 1.0$ equiv), 2 ( $0.40 \mathrm{mmol}, 2.0$ equiv), base $(0.4 \mathrm{mmol})$, triethylamine (3, 0.4 $\mathrm{mmol})$, solvent $(2 \mathrm{~mL}), \mathrm{rt}$, Ar atmosphere. The mixtures were then stirred rapidly and irradiated with a $36 \mathrm{~W}$ Blue LED (approximately $2 \mathrm{~cm}$ away from the light source) at room temperature. After $2 \mathrm{~h}$, the Blue LED was turned off, and one vial was removed from the irradiation setup for analysis. The remaining seven vials were stirred in the absence of light for an additional $2 \mathrm{~h}$. Then, one vial was removed for analysis, and the Blue LED was turned back on to irradiate the remaining six reaction mixtures. After an additional $2 \mathrm{~h}$ of irradiation, the Blue LED was turned off, and one vial was removed for analysis. The remaining five vials were stirred in the absence of light for an additional $2 \mathrm{~h}$. Then, a vial was removed for analysis, and the Blue LED was turned back on to irradiate the remaining four reaction mixtures. After $2 \mathrm{~h}$, the Blue LED was turned off, and one vial was removed for analysis. The remaining three vials were stirred in the absence of light for an additional $2 \mathrm{~h}$, then, a vial was removed for analysis and the Blue LED was turned back on to irradiate the remaining two reaction mixtures. After $2 \mathrm{~h}$, the Blue LED was turned off, and one vial was removed for analysis. The last vial was stirred in the absence of light for an additional $2 \mathrm{~h}$, and then it was analyzed. The yield was determined by ${ }^{19} \mathrm{~F}$ NMR spectroscopy using fluorobenzene as an internal standard.

B) light/dark experiment

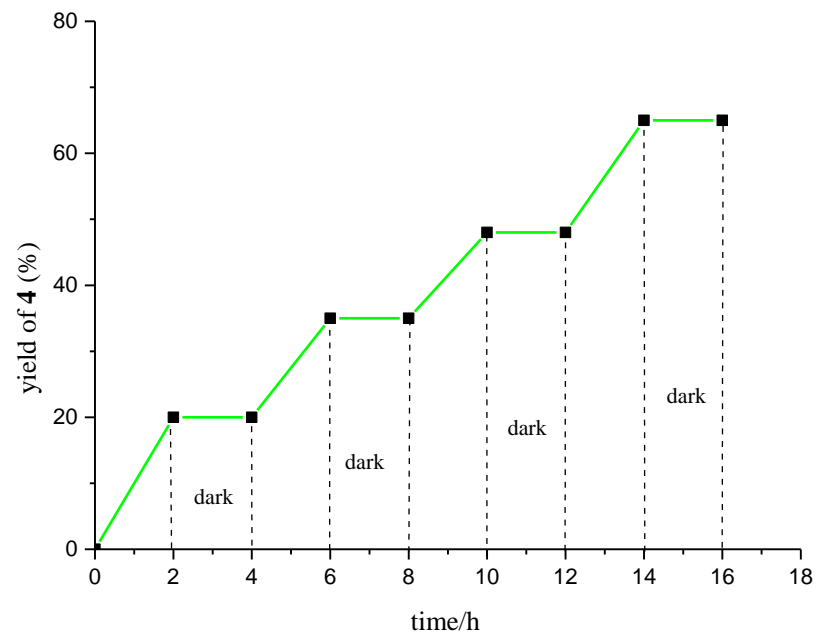

Figure S4 Light/dark experiment.

\subsection{Emission Quenching Experiments (Stern-Volmer Studies)}

Emission intensities were recorded using a CARY VARIAN luminescence spectrophotometer. All

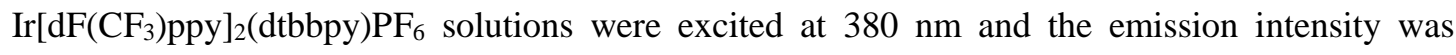
collected at $470 \mathrm{~nm}$. In a typical experiment, to a $3 \times 10^{-6} \mathrm{M}$ solution of $\operatorname{Ir}\left[\mathrm{dF}_{(}\left(\mathrm{CF}_{3}\right) \mathrm{ppy}\right]_{2}(\mathrm{dtbbpy}) \mathrm{PF}_{6}$ in DCE was added the appropriate amount of a quencher in a 
screw-top quartz cuvette. After degassing the sample with a stream of argon for 10 minutes, the emission of the sample was collected.

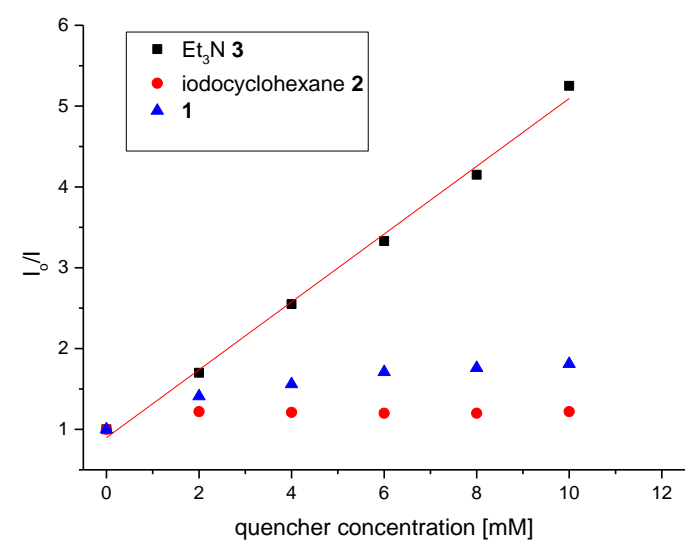

\subsection{Electron donor Experiments}

To a $10 \mathrm{~mL}$ glass vial was added $\operatorname{Ir}\left[\mathrm{dF}\left(\mathrm{CF}_{3}\right) \text { ppy) }\right]_{2}(\mathrm{dtbbpy}) \mathrm{PF}_{6}(2.24 \mathrm{mg}, 0.002 \mathrm{mmol}, 1 \mathrm{~mol} \%), 1$ (0.2 mmol, 1.0 equiv), 2 ( $0.4 \mathrm{mmol}, 2.0$ equiv), base $(0.4 \mathrm{mmol})$, electron donor $(0.4 \mathrm{mmol})$, solvent $(2 \mathrm{~mL}), \mathrm{rt}, \mathrm{Ar}$ atmosphere. The mixture was then stirred rapidly and irradiated with a $36 \mathrm{~W}$ Blue LED (approximately $2 \mathrm{~cm}$ away from the light source) at room temperature for $24 \mathrm{~h}$. The corresponding alkylated product 4 was observed based on ${ }^{19} \mathrm{~F}$ NMR analysis.

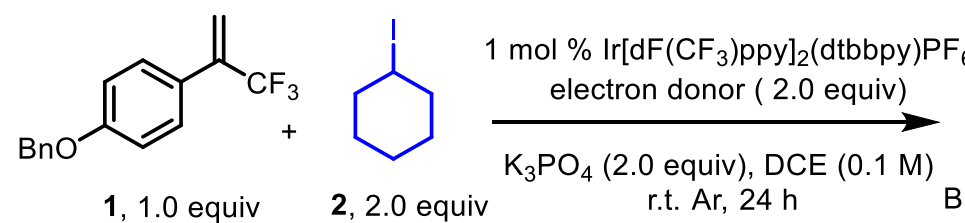

$1,1.0$ equiv
2, 2.0 equiv r.t. Ar, $24 \mathrm{~h}$<smiles>Oc1ccc(C(CC2CCCCC2)=C(F)F)cc1</smiles>

4, $0 \%$<smiles>CCOC(=O)C1=C(C)NC(C)=C(C(=O)OCC)C1</smiles>

trace<smiles>CC1(C)CCCC(C)(C)N1</smiles>

NR

\section{Experimental Procedures and Product Characterization}

\subsection{General Procedure for the difluoroallylation.}

To a $10 \mathrm{~mL}$ glass vial was added $\operatorname{Ir}\left[\mathrm{dF}\left(\mathrm{CF}_{3}\right)\right.$ ppy $\left.)\right]_{2}(\mathrm{dtbbpy}) \mathrm{PF}_{6}(2.24 \mathrm{mg}, 0.002 \mathrm{mmol}, 1 \mathrm{~mol} \%)$, $\alpha$-trifluoromethyl arylalkene ( $0.2 \mathrm{mmol}, 1.0$ equiv), alkyl iodide ( $0.40 \mathrm{mmol}, 2.0$ equiv), base ( 0.4 mmol), triethylamine $(3,0.4 \mathrm{mmol})$, solvent $(2 \mathrm{~mL}), \mathrm{rt}$, Ar atmosphere. The mixture was then stirred rapidly and irradiated with a $36 \mathrm{~W}$ Blue LED (approximately $2 \mathrm{~cm}$ away from the light source) at room temperature for $24 \mathrm{~h}$. The reaction mixture was diluted with $10 \mathrm{~mL}$ of aqueous 1 $\mathrm{M} \mathrm{NaHCO}_{3}$ solution, and extracted with DCM $(3 \times 20 \mathrm{~mL})$. The combined organic extracts were washed with brine $(40 \mathrm{~mL})$, dried over $\mathrm{Na}_{2} \mathrm{SO}_{4}$, and concentrated in vacuo. Purification of the crude product by flash chromatography on silica gel using the indicated solvent system afforded 
the desired product.

\subsection{Product Characterization}

\section{1-(benzyloxy)-4-(3-cyclohexyl-1,1-difluoroprop-1-en-2-yl)benzene (4)}<smiles>FC(F)=C(CC1CCCCC1)c1ccc(OCc2ccccc2)cc1</smiles>

According to the general procedure.

Yellow oil (61.5 mg, 90\%).

$R_{\mathrm{f}} 0.80$ (Petroleum ether).

${ }^{1} \mathbf{H}$ NMR $\left(400 \mathrm{MHz}, \mathrm{CDCl}_{3}\right) \delta 7.73-7.56(\mathrm{~m}, 5 \mathrm{H}), 7.52-7.47(\mathrm{~m}, 2 \mathrm{H}), 7.23(\mathrm{~d}, J=8.7 \mathrm{~Hz}, 2 \mathrm{H})$, $5.33(\mathrm{~s}, 2 \mathrm{H}), 2.56-2.46(\mathrm{~m}, 2 \mathrm{H}), 1.92(\mathrm{dd}, J=21.5,13.3 \mathrm{~Hz}, 5 \mathrm{H}), 1.58-1.47(\mathrm{~m}, 1 \mathrm{H}), 1.39-1.17$ $(\mathrm{m}, 5 \mathrm{H}) .{ }^{13} \mathrm{C}$ NMR $\left(100 \mathrm{MHz}, \mathrm{CDCl}_{3}\right) \delta 157.8,136.9,129.4\left(\mathrm{t}, J_{\mathrm{C}-\mathrm{F}}=3.2 \mathrm{~Hz}\right), 129.3,128.6,128.0$, 127.5, 127.5, $90.4\left(\mathrm{dd}, J_{\mathrm{C}-\mathrm{F}}=22.0,12.9 \mathrm{~Hz}\right), 70.0,35.6,35.3,32.8,26.4,26.1$.

${ }^{19}$ F NMR $\left(376 \mathrm{MHz}, \mathrm{CDCl}_{3}\right) \delta-92.17(\mathrm{~d}, J=46.7 \mathrm{~Hz}, 1 \mathrm{~F}),-92.59$ (d, $\left.J=46.4 \mathrm{~Hz}, 1 \mathrm{~F}\right)$.

HRMS (EI) m/z: [M] $]^{+}$Calcd for $\mathrm{C}_{22} \mathrm{H}_{24} \mathrm{~F}_{2} \mathrm{O}$ 342.1795; found 342.1790.

\section{1-(benzyloxy)-4-(1,1-difluoropent-1-en-2-yl)benzene (5).}<smiles>CCCC(=C(F)F)c1ccc(OCc2ccccc2)cc1</smiles>

According to the general procedure.

Yellow oil (30.0 mg, 52\%).

$R_{\mathrm{f}} 0.50$ (Petroleum ether).

${ }^{1} \mathbf{H}$ NMR $\left(400 \mathrm{MHz}, \mathrm{CDCl}_{3}\right) \delta 7.49-7.37(\mathrm{~m}, 5 \mathrm{H}), 7.26(\mathrm{~d}, J=8.6 \mathrm{~Hz}, 2 \mathrm{H}), 7.02-6.96(\mathrm{~m}, 2 \mathrm{H})$, $5.09(\mathrm{~s}, 2 \mathrm{H}), 2.44-2.30(\mathrm{~m}, 2 \mathrm{H}), 1.41(\mathrm{dq}, J=14.8,7.4 \mathrm{~Hz}, 2 \mathrm{H}), 0.92(\mathrm{t}, J=7.4 \mathrm{~Hz}, 3 \mathrm{H}) .{ }^{13} \mathrm{C}$ NMR $\left(100 \mathrm{MHz}, \mathrm{CDCl}_{3}\right) \delta 157.8,136.9,129.4\left(\mathrm{t}, J_{\mathrm{C}-\mathrm{F}}=3.4 \mathrm{~Hz}\right), 128.6,128.0,127.5,114.7,91.6$ $\left(\mathrm{dd}, J_{\mathrm{C}-\mathrm{F}}=20.2,14.5 \mathrm{~Hz}\right), 70.0,29.6,20.8,13.4$.

${ }^{19}$ F NMR $\left(376 \mathrm{MHz}, \mathrm{CDCl}_{3}\right) \delta-92.72(\mathrm{~d}, J=47.0 \mathrm{~Hz}),-92.87(\mathrm{~d}, J=47.0 \mathrm{~Hz})$.

HRMS (ESI) m/z: [M + H] ${ }^{+}$Calcd for $\mathrm{C}_{18} \mathrm{H}_{19} \mathrm{~F}_{2} \mathrm{O}$ 289.1398; found 289.1396 .

1-(benzyloxy)-4-(1,1-difluorohept-1-en-2-yl)benzene (6).<smiles>CCCCCC(=C(F)F)c1ccc(OCc2ccccc2)cc1</smiles>

According to the general procedure.

White oil (29.7 mg, 47\%).

$R_{\mathrm{f}} 0.50$ (Petroleum ether). 
${ }^{1} \mathbf{H}$ NMR $\left(400 \mathrm{MHz}, \mathrm{CDCl}_{3}\right) \delta 7.44-7.32(\mathrm{~m}, 5 \mathrm{H}), 7.23(\mathrm{~d}, J=8.6 \mathrm{~Hz}, 2 \mathrm{H}), 6.96(\mathrm{~d}, J=8.5 \mathrm{~Hz}$, $2 \mathrm{H}), 5.06(\mathrm{~s}, 2 \mathrm{H}), 2.34(\mathrm{dd}, J=9.8,4.7 \mathrm{~Hz}, 2 \mathrm{H}), 1.34(\mathrm{dd}, J=13.3,6.3 \mathrm{~Hz}, 2 \mathrm{H}), 1.26(\mathrm{dd}, J=8.5$, $5.3 \mathrm{~Hz}, 4 \mathrm{H}), 0.85(\mathrm{t}, J=6.6 \mathrm{~Hz}, 3 \mathrm{H}) .{ }^{13} \mathbf{C} \mathbf{N M R}\left(100 \mathrm{MHz}, \mathrm{CDCl}_{3}\right) \delta 157.8,136.9,129.4\left(\mathrm{t}, J_{\mathrm{C}-\mathrm{F}}=\right.$ $3.7 \mathrm{~Hz}), 128.6,128.0,127.5,126.2,114.7,91.8\left(\mathrm{dd}, J_{\mathrm{C}-\mathrm{F}}=25.3,17.0 \mathrm{~Hz}\right), 70.0,31.2,27.6,27.4$, 22.3, 14.0. ${ }^{19} \mathbf{F}$ NMR $\left(376 \mathrm{MHz}, \mathrm{CDCl}_{3}\right) \delta-92.69(\mathrm{~d}, J=43.9 \mathrm{~Hz}),-92.86(\mathrm{~d}, J=44.0 \mathrm{~Hz})$.

HRMS (ESI) m/z: [M + H] ${ }^{+}$Calcd for $\mathrm{C}_{20} \mathrm{H}_{23} \mathrm{~F}_{2} \mathrm{O}$ 317.1711; found 317.1711.

1-(benzyloxy)-4-(1,1-difluoro-5-methylhex-1-en-2-yl)benzene (7).<smiles>CC(C)CCC(=C(F)F)c1ccc(OBr)cc1</smiles>

According to the general procedure.

Yellow oil (35.4 mg, 56\%).

$R_{\mathrm{f}} 0.70$ (Petroleum ether).

${ }^{1} \mathbf{H}$ NMR $\left(400 \mathrm{MHz}, \mathrm{CDCl}_{3}\right) \delta 7.38(\mathrm{ddd}, J=17.0,11.0,4.8 \mathrm{~Hz}, 5 \mathrm{H}), 7.22(\mathrm{~d}, J=8.7 \mathrm{~Hz}, 2 \mathrm{H})$, $6.96(\mathrm{~d}, J=8.7 \mathrm{~Hz}, 2 \mathrm{H}), 5.08-5.02(\mathrm{~m}, 2 \mathrm{H}), 2.42-2.25(\mathrm{~m}, 2 \mathrm{H}), 1.57-1.52(\mathrm{~m}, 1 \mathrm{H}), 1.26-$ $1.20(\mathrm{~m}, 2 \mathrm{H}), 0.87(\mathrm{~d}, J=6.6 \mathrm{~Hz}, 6 \mathrm{H}) .{ }^{13} \mathbf{C} \mathbf{~ N M R}\left(100 \mathrm{MHz}, \mathrm{CDCl}_{3}\right) \delta 157.8,136.9,129.3\left(\mathrm{t}, J_{\mathrm{C}-\mathrm{F}}\right.$ $=3.7 \mathrm{~Hz}), 128.6,128.6,128.0,127.6,127.5,127.5,126.2,114.7,114.6,92.0\left(\mathrm{dd}, J_{\mathrm{C}-\mathrm{F}}=18.1\right.$, $15.3 \mathrm{~Hz}), 70.0,36.8,29.7,27.6,25.6,22.4 .{ }^{19} \mathbf{F} \mathbf{~ N M R}\left(376 \mathrm{MHz}, \mathrm{CDCl}_{3}\right) \delta-92.91$ (s, 2F).

HRMS (ESI-) m/z: $[\mathrm{M}+\mathrm{H}]^{+}$Calcd for $\mathrm{C}_{20} \mathrm{H}_{23} \mathrm{~F}_{2} \mathrm{O} 317.1711$; found 317.1710.

1-(benzyloxy)-4-(4-chloro-1,1-difluorobut-1-en-2-yl)benzene (8).<smiles>O=C(Oc1ccc(C(CCCl)=C(F)F)cc1)c1ccccc1</smiles>

According to the general procedure.

Yellow oil (32.6 mg, 53\%).

$R_{\mathrm{f}} 0.80$ (Petroleum ether).

${ }^{1}$ H NMR $\left(400 \mathrm{MHz}, \mathrm{CDCl}_{3}\right) \delta 7.74-7.55(\mathrm{~m}, 5 \mathrm{H}), 7.48(\mathrm{~d}, J=8.2 \mathrm{~Hz}, 2 \mathrm{H}), 7.25-7.20(\mathrm{~m}, 2 \mathrm{H})$, $5.32(\mathrm{~s}, 2 \mathrm{H}), 3.71(\mathrm{t}, J=7.1 \mathrm{~Hz}, 2 \mathrm{H}), 3.07(\mathrm{tt}, J=7.1,2.1 \mathrm{~Hz}, 2 \mathrm{H}) .{ }^{13} \mathbf{C ~ N M R}\left(100 \mathrm{MHz}, \mathrm{CDCl}_{3}\right) \delta$ $158.2,154.3\left(\mathrm{dd}, J_{\mathrm{C}-\mathrm{F}}=294.5,287.3 \mathrm{~Hz}\right), 136.7,129.5\left(\mathrm{t}, J_{\mathrm{C}-\mathrm{F}}=2.9 \mathrm{~Hz}\right), 128.6,128.0,127.5$, 124.6, 115.0, $89.0\left(\mathrm{dd}, J_{\mathrm{C}-\mathrm{F}}=22.1,15.8 \mathrm{~Hz}\right), 70.0,41.7,31.4 .{ }^{19} \mathbf{F}$ NMR $\left(376 \mathrm{MHz}, \mathrm{CDCl}_{3}\right) \delta$ $-90.11(\mathrm{~d}, J=40.7 \mathrm{~Hz}, 1 \mathrm{~F}),-90.58(\mathrm{~d}, J=40.9 \mathrm{~Hz}, 1 \mathrm{~F})$.

HRMS (ESI) m/z: [M+ H] $]^{+}$Calcd for $\mathrm{C}_{17} \mathrm{H}_{16} \mathrm{ClF}_{2} \mathrm{O}$ 309.0852; found 309.0851.

1-(benzyloxy)-4-(1,1-difluoro-4-methylpent-1-en-2-yl)benzene (9). 
<smiles>CC(C)CC(=C(F)F)c1ccc(OCc2ccccc2)cc1</smiles>

According to the general procedure.

Colorless oil (47.1 mg, 78\%).

$R_{\mathrm{f}} 0.80$ (Petroleum ether).

${ }^{1} \mathbf{H}$ NMR $\left(400 \mathrm{MHz}, \mathrm{CDCl}_{3}\right) \delta 7.42-7.33(\mathrm{~m}, 5 \mathrm{H}), 7.22(\mathrm{~d}, J=8.1 \mathrm{~Hz}, 2 \mathrm{H}), 6.95(\mathrm{~d}, J=8.7 \mathrm{~Hz}$, $2 \mathrm{H}), 5.05(\mathrm{~s}, 2 \mathrm{H}), 2.30-2.16(\mathrm{~m}, 2 \mathrm{H}), 1.60-1.53(\mathrm{~m}, 1 \mathrm{H}), 0.86(\mathrm{~d}, J=6.6 \mathrm{~Hz}, 6 \mathrm{H})$.

.${ }^{13} \mathrm{C}$ NMR $\left(100 \mathrm{MHz}, \mathrm{CDCl}_{3}\right) \delta 157.8,136.9,128.9\left(\mathrm{t}, J_{\mathrm{C}-\mathrm{F}}=3.2 \mathrm{~Hz}\right), 128.6,127.5,114.7$, 91.1(dd, $\left.J_{\mathrm{C}-\mathrm{F}}=20 \backslash 1.1,13.2 \mathrm{~Hz}\right), 70.0,36.7,26.3,22.0$.

${ }^{19} \mathbf{F}$ NMR $\left(376 \mathrm{MHz}, \mathrm{CDCl}_{3}\right) \delta-92.46(\mathrm{~d}, J=46.2 \mathrm{~Hz}, 1 \mathrm{~F}),-92.85(\mathrm{~d}, J=46.3 \mathrm{~Hz}, 1 \mathrm{~F})$.

HRMS (ESI) $\mathrm{m} / \mathrm{z}$ : $[\mathrm{M}+\mathrm{H}]^{+}$Calcd for $\mathrm{C}_{19} \mathrm{H}_{20} \mathrm{~F}_{2} \mathrm{O}$ 303.1555; found 303.1554 .

(S)-1-(benzyloxy)-4-(1,1-difluoro-4-methylhex-1-en-2-yl)benzene (10).<smiles>CCC(C)CC(=C(F)F)c1ccc(OBr)cc1</smiles>

According to the general procedure.

Colorless oil (50.6 mg, 80\%).

$R_{\mathrm{f}} 0.80$ (Petroleum ether).

${ }^{1} \mathbf{H}$ NMR $\left(400 \mathrm{MHz}, \mathrm{CDCl}_{3}\right) \delta 7.48-7.37(\mathrm{~m}, 5 \mathrm{H}), 7.27(\mathrm{~d}, J=8.6 \mathrm{~Hz}, 2 \mathrm{H}), 7.00(\mathrm{~d}, J=8.7 \mathrm{~Hz}$, $2 \mathrm{H}), 5.10(\mathrm{~s}, 2 \mathrm{H}), 2.44-2.15(\mathrm{~m}, 2 \mathrm{H}), 1.40(\mathrm{dt}, J=16.5,6.0 \mathrm{~Hz}, 2 \mathrm{H}), 1.21-1.14(\mathrm{~m}, 1 \mathrm{H}), 0.90$ $0.85(\mathrm{~m}, 6 \mathrm{H}) .{ }^{13} \mathrm{C}$ NMR $\left(100 \mathrm{MHz}, \mathrm{CDCl}_{3}\right) \delta 157.8,153.8\left(\mathrm{dd}, J_{\mathrm{C}-\mathrm{F}}=286.3,284.2 \mathrm{~Hz}\right), 136.9$, $129.4\left(\mathrm{t}, J_{\mathrm{C}-\mathrm{F}}=3.1 \mathrm{~Hz}\right), 128.6,128.0,127.5,114.7,91.0\left(\mathrm{dd}, J_{\mathrm{C}-\mathrm{F}}=21.5,12.7 \mathrm{~Hz}\right), 70.0,34.7$, 32.6, 29.0, 18.6, 11.2. ${ }^{19} \mathbf{F}$ NMR $\left(376 \mathrm{MHz}, \mathrm{CDCl}_{3}\right) \delta-92.47$ (d, $\left.J=46.7 \mathrm{~Hz}, 1 \mathrm{~F}\right),-92.70(\mathrm{~d}, J=$ $46.7 \mathrm{~Hz}, 1 \mathrm{~F})$.

HRMS (ESI) m/z: $[\mathrm{M}+\mathrm{H}]^{+}$Calcd for $\mathrm{C}_{20} \mathrm{H}_{23} \mathrm{~F}_{2} \mathrm{O} 317.1711$; found 317.1713 .

1-isopropyl-6,7-dimethoxyisoquinoline (11).<smiles>CCCC(CC)CC(=C(F)F)c1ccc(OCc2ccccc2)cc1</smiles>

According to the general procedure.

Yellow oil (57.8 mg, 84\%).

$R_{\mathrm{f}} 0.80$ (Petroleum ether).

${ }^{1} \mathbf{H}$ NMR $\left(400 \mathrm{MHz}, \mathrm{CDCl}_{3}\right) \delta 7.37(\mathrm{ddd}, J=23.5,14.9,7.0 \mathrm{~Hz}, 5 \mathrm{H}), 7.25-7.18(\mathrm{~m}, 2 \mathrm{H}), 6.95(\mathrm{~d}$, $J=8.8 \mathrm{~Hz}, 2 \mathrm{H}), 5.05(\mathrm{~s}, 2 \mathrm{H}), 2.28-2.23(\mathrm{~m}, 2 \mathrm{H}), 1.33-1.15(\mathrm{~m}, 7 \mathrm{H}), 0.87-0.74(\mathrm{~m}, 6 \mathrm{H}) .{ }^{13} \mathrm{C}$ NMR $\left(100 \mathrm{MHz}, \mathrm{CDCl}_{3}\right) \delta 157.8,136.9,129.4\left(\mathrm{t}, J_{\mathrm{C}-\mathrm{F}}=3.2 \mathrm{~Hz}\right), 128.6,128.0,127.5,114.7,91.2$, 
91.1(dd, $\left.J_{\mathrm{C}-\mathrm{F}}=20.8,12.6 \mathrm{~Hz}\right), 91.0,90.9,70.0,36.5,34.8,31.7,25.3,19.5,14.4,10.4 .{ }^{19} \mathbf{F}$ NMR $\left(376 \mathrm{MHz}, \mathrm{CDCl}_{3}\right) \delta-92.50(\mathrm{~d}, J=47.0 \mathrm{~Hz}, 1 \mathrm{~F}),-92.68(\mathrm{~d}, J=47.1 \mathrm{~Hz}, 1 \mathrm{~F})$.

HRMS (ESI) m/z: [M + H] ${ }^{+}$Calcd for $\mathrm{C}_{22} \mathrm{H}_{27} \mathrm{~F}_{2} \mathrm{O}$ 345.2024; found 345.2023.

(S)-1-(benzyloxy)-4-(4-ethyl-1,1-difluoronon-1-en-2-yl)benzene (12).<smiles>CCCCCC(CC)CC(=C(F)F)c1ccc(OCc2ccccc2)cc1</smiles>

According to the general procedure.

Colorless oil (61.8 mg, 83\%).

$R_{\mathrm{f}} 0.70$ (Petroleum ether).

${ }^{1} \mathbf{H}$ NMR $\left(400 \mathrm{MHz}, \mathrm{CDCl}_{3}\right) \delta 7.46-7.30(\mathrm{~m}, 5 \mathrm{H}), 7.22(\mathrm{~d}, J=7.1 \mathrm{~Hz}, 2 \mathrm{H}), 6.96(\mathrm{dd}, J=8.7,2.2$ $\mathrm{Hz}, 2 \mathrm{H}), 5.05$ (s, 2H), $2.28(\mathrm{~s}, 2 \mathrm{H}), 1.32-1.16(\mathrm{~m}, 11 \mathrm{H}), 0.89-0.77(\mathrm{~m}, 6 \mathrm{H}) .{ }^{13} \mathrm{C}$ NMR $(100$ $\left.\mathrm{MHz}, \mathrm{CDCl}_{3}\right) \delta{ }^{13} \mathrm{C} \mathrm{NMR}\left(100 \mathrm{MHz}, \mathrm{CDCl}_{3}\right) \delta 157.8,136.9,129.4\left(\mathrm{t}, J_{\mathrm{C}-\mathrm{F}}=3.0 \mathrm{~Hz}\right), 128.6,128.0$, 127.5, 126.3, 114.7, $91.1\left(\mathrm{dd}, J_{\mathrm{C}-\mathrm{F}}=20.3,14.0 \mathrm{~Hz}\right), 77.3,77.0,76.7,70.0,36.7,32.3,32.2,31.7$, 25.9, 25.3, 22.6, 14.1, 10.4. ${ }^{19} \mathbf{F}$ NMR $\left(376 \mathrm{MHz}, \mathrm{CDCl}_{3}\right) \delta-92.51(\mathrm{~d}, J=47.0 \mathrm{~Hz}, 1 \mathrm{~F}),-92.66(\mathrm{~d}$, $J=47.1 \mathrm{~Hz}, 1 \mathrm{~F})$.

HRMS (ESI) m/z: [M + H] $]^{+}$Calcd for $\mathrm{C}_{24} \mathrm{H}_{31} \mathrm{~F}_{2} \mathrm{O}$ 373.2337; found 373.2334.

\section{1-(benzyloxy)-4-(4-butyl-1,1-difluorooct-1-en-2-yl)benzene (13).}<smiles>CCCCC(CCCC)CC(=C(F)F)c1ccc(OCc2ccccc2)cc1</smiles>

According to the general procedure.

Colorless oil (65.6 mg, 85\%).

$R_{\mathrm{f}} 0.50$ (Petroleum ether).

${ }^{1} \mathbf{H}$ NMR $\left(400 \mathrm{MHz}, \mathrm{CDCl}_{3}\right) \delta 7.44-7.32(\mathrm{~m}, 5 \mathrm{H}), 7.24-7.19(\mathrm{~m}, 2 \mathrm{H}), 6.95(\mathrm{~d}, J=7.2 \mathrm{~Hz}, 2 \mathrm{H})$, $5.05(\mathrm{~s}, 2 \mathrm{H}), 2.32-2.24(\mathrm{~m}, 2 \mathrm{H}), 1.24-1.14(\mathrm{~m}, 13 \mathrm{H}), 0.87-0.83(\mathrm{~m}, 6 \mathrm{H}) .{ }^{13} \mathbf{C} \mathbf{N M R}(100 \mathrm{MHz}$, $\left.\mathrm{CDCl}_{3}\right) \delta 157.8,136.9,129.4\left(\mathrm{t}, J_{\mathrm{C}-\mathrm{F}}=3.4 \mathrm{~Hz}\right), 128.6,128.0,127.5,126.3,114.7,91.1\left(\mathrm{dd}, J_{\mathrm{C}-\mathrm{F}}=\right.$ 19.0, $15.0 \mathrm{~Hz}), 70.0,35.3,32.6,32.1,28.4,23.0,14.1 .{ }^{19} \mathbf{F} \mathbf{N M R}\left(376 \mathrm{MHz}, \mathrm{CDCl}_{3}\right) \boldsymbol{\delta}-92.49-$ $-92.80(\mathrm{~m}, 2 \mathrm{~F})$.

HRMS (EI-TOF) calcd for $\mathrm{C}_{25} \mathrm{H}_{32} \mathrm{~F}_{2} \mathrm{O}[\mathrm{M}]^{+} 386.2421$, found 386.2415 .

3-(2-(4-(benzyloxy)phenyl)-3,3-difluoroallyl)oxetane (14).<smiles>FC(F)=C(CC1COC1)c1ccc(OCc2ccccc2)cc1</smiles>

According to the general procedure. 
Colorless oil (44.2 mg, 70\%).

$R_{\mathrm{f}} 0.60$ (Petroleum ether).

${ }^{1}$ H NMR $\left(400 \mathrm{MHz}, \mathrm{CDCl}_{3}\right) \delta 7.46-7.31(\mathrm{~m}, 5 \mathrm{H}), 7.15(\mathrm{~d}, J=8.1 \mathrm{~Hz}, 2 \mathrm{H}), 6.99-6.92(\mathrm{~m}, 2 \mathrm{H})$, $5.06(\mathrm{~s}, 2 \mathrm{H}), 4.70-4.61(\mathrm{~m}, 2 \mathrm{H}), 4.33(\mathrm{dd}, J=8.6,3.6 \mathrm{~Hz}, 2 \mathrm{H}), 3.00(\mathrm{dt}, J=14.2,7.1 \mathrm{~Hz}, 1 \mathrm{H})$, $2.75(\mathrm{dd}, J=7.6,1.6 \mathrm{~Hz}, 2 \mathrm{H}) .{ }^{13} \mathbf{C}$ NMR $\left(100 \mathrm{MHz}, \mathrm{CDCl}_{3}\right) \delta 158.1,136.7,129.4\left(\mathrm{t}, J_{\mathrm{C}-\mathrm{F}}=3.4\right.$ $\mathrm{Hz}), 128.6,128.1,127.5,114.9,76.8,70.0,33.7,31.8 .{ }^{19} \mathbf{F} \mathbf{~ N M R}\left(376 \mathrm{MHz}, \mathrm{CDCl}_{3}\right) \delta-91.43(\mathrm{~d}, J$ $=44.8 \mathrm{~Hz}, 1 \mathrm{~F}),-91.94(\mathrm{~d}, J=44.8 \mathrm{~Hz}, 1 \mathrm{~F})$.

HRMS (ESI) m/z: [M + H] $]^{+}$Calcd for $\mathrm{C}_{19} \mathrm{H}_{19} \mathrm{~F}_{2} \mathrm{O}_{2}$ 317.1348; found 317.1343.

tert-butyl 3-(2-(4-(benzyloxy)phenyl)-3,3-difluoroallyl)azetidine-1-carboxylate (15).<smiles>CC(=O)N1CC(CC(=C(F)F)c2ccc(OCc3ccccc3)cc2)C1</smiles>

According to the general procedure.

Colorless oil (49.8 mg, 60\%).

$R_{\mathrm{f}} 0.70$ (Petroleum ether/EtOAc, 7/1).

${ }^{1}$ H NMR $\left(400 \mathrm{MHz}, \mathrm{CDCl}_{3}\right) \delta 7.42-7.32(\mathrm{~m}, 6 \mathrm{H}), 7.13(\mathrm{~d}, J=8.4 \mathrm{~Hz}, 1 \mathrm{H}), 6.97-6.90(\mathrm{~m}, 2 \mathrm{H})$, $5.04(\mathrm{~s}, 2 \mathrm{H}), 3.86(\mathrm{t}, J=8.4 \mathrm{~Hz}, 2 \mathrm{H}), 3.51(\mathrm{dd}, J=8.7,5.5 \mathrm{~Hz}, 2 \mathrm{H}), 2.61(\mathrm{~d}, J=7.7 \mathrm{~Hz}, 2 \mathrm{H}), 1.41$ - 1.37 (m, 9H). ${ }^{13} \mathbf{C}$ NMR $\left(100 \mathrm{MHz}, \mathrm{CDCl}_{3}\right) \delta 158.2,156.4,136.7,129.4,128.6,128.0,127.5$, 126.0, 114.9, 114.8, 79.8, 79.6, 70.0, 53.9, 32.5, 28.4, 27.3. ${ }^{19} \mathbf{F}$ NMR $\left(376 \mathrm{MHz}, \mathrm{CDCl}_{3}\right) \delta-91.20$ $(\mathrm{d}, J=44.5 \mathrm{~Hz}, 1 \mathrm{~F}),-91.81(\mathrm{~d}, J=44.5 \mathrm{~Hz}, 1 \mathrm{~F})$.

HRMS (ESI) m/z: [M + Na ${ }^{+}$Calcd for $\mathrm{C}_{24} \mathrm{H}_{27} \mathrm{~F}_{2} \mathrm{NO}_{3} \mathrm{Na} 438.1851$; found 438.1847.

1-(benzyloxy)-4-(3-cyclopentyl-1,1-difluoroprop-1-en-2-yl)benzene (16).<smiles>FC(F)=C(CC1CCCC1)c1ccc(OCc2ccccc2)cc1</smiles>

According to the general procedure.

Colorless oil (49.2 mg, 75\%).

$R_{\mathrm{f}} 0.65$ (Petroleum ether).

${ }^{1} \mathbf{H}$ NMR (400 MHz, $\left.\mathrm{CDCl}_{3}\right) \delta 7.47-7.32(\mathrm{~m}, 5 \mathrm{H}), 7.26-7.18(\mathrm{~m}, 2 \mathrm{H}), 6.99-6.90(\mathrm{~m}, 2 \mathrm{H}), 5.05$ (s, 2H), $2.41-2.22(\mathrm{~m}, 2 \mathrm{H}), 1.80-1.65(\mathrm{~m}, 1 \mathrm{H}), 1.61-1.09(\mathrm{~m}, 8 \mathrm{H}) .{ }^{13} \mathbf{C}$ NMR (100 MHz, $\left.\mathrm{CDCl}_{3}\right) \delta 157.8,136.9,129.5\left(\mathrm{t}, J_{\mathrm{C}-\mathrm{F}}=3.1 \mathrm{~Hz}\right), 128.6,128.0,127.5,91.7\left(\mathrm{dd}, J_{\mathrm{C}-\mathrm{F}}=22.0,13.0\right.$ $\mathrm{Hz}), 70.0,38.2,33.6,32.1,25.0 .{ }^{19} \mathbf{F}$ NMR $\left(376 \mathrm{MHz}, \mathrm{CDCl}_{3}\right) \delta-92.94(\mathrm{~d}, J=47.5 \mathrm{~Hz}, 1 \mathrm{~F})$, $-93.26(\mathrm{~d}, J=47.4 \mathrm{~Hz}, 1 \mathrm{~F})$.

HRMS (ESI) m/z: [M + H] ${ }^{+}$Calcd for $\mathrm{C}_{21} \mathrm{H}_{23} \mathrm{~F}_{2} \mathrm{O}$ 329.1711; found 329.1710.

4-(2-(4-(benzyloxy)phenyl)-3,3-difluoroallyl)tetrahydro-2H-pyran (17). 
<smiles>FC(F)=C(CC1CCOCC1)c1ccc(Br)cc1</smiles>

According to the general procedure.

Colorless oil (56.4 mg, 82\%).

$R_{\mathrm{f}} 0.65$ (Petroleum ether/EtOAc, 10/1).

${ }^{1} \mathbf{H}$ NMR $\left(400 \mathrm{MHz}, \mathrm{CDCl}_{3}\right) \delta 7.42(\mathrm{ddd}, J=23.4,13.9,7.0 \mathrm{~Hz}, 5 \mathrm{H}), 7.26(\mathrm{~d}, J=8.3 \mathrm{~Hz}, 2 \mathrm{H})$, $7.01(\mathrm{~d}, J=8.7 \mathrm{~Hz}, 2 \mathrm{H}), 5.10(\mathrm{~s}, 2 \mathrm{H}), 3.96-3.93(\mathrm{~m}, 2 \mathrm{H}), 3.30(\mathrm{t}, J=11.1 \mathrm{~Hz}, 2 \mathrm{H}), 2.35(\mathrm{~d}, J=$ $6.5 \mathrm{~Hz}, 2 \mathrm{H}), 1.65-1.52(\mathrm{~m}, 3 \mathrm{H}), 1.41-1.31(\mathrm{~m}, 2 \mathrm{H}) .{ }^{13} \mathbf{C} \mathbf{~ N M R}\left(100 \mathrm{MHz}, \mathrm{CDCl}_{3}\right) \delta 157.9$, $153.9\left(\mathrm{dd}, J_{\mathrm{C}-\mathrm{F}}=288.2,284.1 \mathrm{~Hz}\right), 136.8,129.3\left(\mathrm{t}, J_{\mathrm{C}-\mathrm{F}}=3.1 \mathrm{~Hz}\right), 128.6,128.0,127.5,114.8$, $89.8\left(\mathrm{dd}, J_{\mathrm{C}-\mathrm{F}}=21.5,14.0 \mathrm{~Hz}\right), 70.0,67.8,34.8,33.1,32.6 .{ }^{19} \mathbf{F} \mathbf{~ N M R}\left(376 \mathrm{MHz}, \mathrm{CDCl}_{3}\right) \delta$ $-91.63(\mathrm{~d}, J=45.0 \mathrm{~Hz}, 1 \mathrm{~F}),-91.94(\mathrm{~d}, J=45.2 \mathrm{~Hz}, 1 \mathrm{~F})$.

HRMS (ESI) m/z: [M + H] $]^{+}$Calcd for $\mathrm{C}_{21} \mathrm{H}_{23} \mathrm{~F}_{2} \mathrm{O}_{2}$ 345.1661; found 345.1658.

tert-butyl 4-(2-(4-(benzyloxy)phenyl)-3,3-difluoroallyl)piperidine-1-carboxylate (18).<smiles>CC(C)(C)OC(=O)N1CCC(CC(=C(F)F)c2ccc(OCc3ccccc3)cc2)CC1</smiles>

According to the general procedure.

Colorless oil (54.0 mg, 61\%).

$R_{\mathrm{f}} 0.65$ (Petroleum ether/EtOAc, 7/1).

${ }^{1} \mathbf{H}$ NMR $\left(400 \mathrm{MHz}, \mathrm{CDCl}_{3}\right) \delta 7.41(\mathrm{ddd}, J=23.1,13.6,6.8 \mathrm{~Hz}, 5 \mathrm{H}), 7.32-7.23(\mathrm{~m}, 2 \mathrm{H}), 7.00(\mathrm{~d}$, $J=8.3 \mathrm{~Hz}, 2 \mathrm{H}), 5.09(\mathrm{~s}, 2 \mathrm{H}), 4.07-4.06(\mathrm{~m}, 2 \mathrm{H}), 2.63-2.57(\mathrm{~m}, 2 \mathrm{H}), 2.33-2.32(\mathrm{~m}, 2 \mathrm{H}), 1.76$ - $1.62(\mathrm{~m}, 2 \mathrm{H}), 1.47(\mathrm{~s}, 9 \mathrm{H}), 1.31-1.28(\mathrm{~m}, 1 \mathrm{H}), 1.15-1.13(\mathrm{~m}, 2 \mathrm{H}) .{ }^{13} \mathbf{C}$ NMR (100 MHz, $\left.\mathrm{CDCl}_{3}\right) \delta 157.9,154.8,153.9\left(\mathrm{dd}, J_{\mathrm{C}-\mathrm{F}}=284.2,281.1 \mathrm{~Hz}\right), 136.8,129.3\left(\mathrm{t}, J_{\mathrm{C}-\mathrm{F}}=3.0 \mathrm{~Hz}\right), 128.6$, 128.0, 127.5, 125.9, 115.0, 114.8, 89.9(dd, $\left.J_{\mathrm{C}-\mathrm{F}}=21.2,13.2 \mathrm{~Hz}\right), 79.3,70.0,43.6,34.4,34.1$, 31.7, 28.5, 28.4. ${ }^{19}$ F NMR $\left(376 \mathrm{MHz}, \mathrm{CDCl}_{3}\right) \delta-91.6(\mathrm{~d}, J=44.7 \mathrm{~Hz}, 1 \mathrm{~F}),-91.9(\mathrm{~d}, J=45.3 \mathrm{~Hz}$, $1 \mathrm{~F})$.

HRMS (ESI) m/z: [M + H] $]^{+}$Calcd for $\mathrm{C}_{26} \mathrm{H}_{31} \mathrm{~F}_{2} \mathrm{NNaO}_{3}$ 466.2164; found 466.2159.

benzyl 4-(2-(4-(benzyloxy)phenyl)-3,3-difluoroallyl)piperidine-1-carboxylate (19).<smiles>O=C(O)N1CCC(CC(=C(F)F)c2ccc(OCc3ccccc3)cc2)CC1</smiles>

According to the general procedure.

Colorless oil (53.4 mg, 56\%).

$R_{\mathrm{f}} 0.60$ (Petroleum ether/EtOAc, 10/1). 
${ }^{1}$ H NMR $\left(400 \mathrm{MHz}, \mathrm{CDCl}_{3}\right) \delta 7.51-7.34(\mathrm{~m}, 10 \mathrm{H}), 7.25(\mathrm{~d}, J=8.4 \mathrm{~Hz}, 2 \mathrm{H}), 7.01(\mathrm{~d}, J=8.5 \mathrm{~Hz}$, $2 \mathrm{H}), 5.14(\mathrm{~s}, 2 \mathrm{H}), 5.10(\mathrm{~s}, 2 \mathrm{H}), 4.15-4.15(\mathrm{~m}, 2 \mathrm{H}), 2.68-2.67(\mathrm{~m}, 2 \mathrm{H}), 2.35-2.33(\mathrm{~m}, 2 \mathrm{H})$, $1.67-1.65(\mathrm{~m}, 2 \mathrm{H}), 1.47-1.46(\mathrm{~m}, 1 \mathrm{H}), 1.18-1.17(\mathrm{~m}, 2 \mathrm{H}) .{ }^{13} \mathbf{C} \mathbf{~ N M R}\left(100 \mathrm{MHz}, \mathrm{CDCl}_{3}\right) \delta$ 158.0, 155.2, 136.9, 136.8, $129.3\left(\mathrm{t}, J_{\mathrm{C}-\mathrm{F}}=2.9 \mathrm{~Hz}\right), 128.6,128.4,128.1,127.9,127.8,127.5$, $114.8,89.9\left(\mathrm{dd}, J_{\mathrm{C}-\mathrm{F}}=19.5,13.0 \mathrm{~Hz}\right), 70.0,67.0,44.0,34.4,34.1,31.6 .{ }^{19} \mathbf{F}$ NMR $(376 \mathrm{MHz}$, $\left.\mathrm{CDCl}_{3}\right) \delta-91.48(\mathrm{~d}, J=44.7 \mathrm{~Hz}, 1 \mathrm{~F}),-91.81(\mathrm{~d}, J=44.8 \mathrm{~Hz}, 1 \mathrm{~F})$.

HRMS (ESI) m/z: $[\mathrm{M}+\mathrm{H}]^{+}$Calcd for $\mathrm{C}_{29} \mathrm{H}_{30} \mathrm{~F}_{2} \mathrm{NO}_{3}$ 478.2188; found 478.2184.

1-(benzyloxy)-4-(1,1-difluoro-4,4-dimethylpent-1-en-2-yl)benzene (20).<smiles>CC(C)(C)CC(=C(F)F)c1ccc(OCc2ccccc2)cc1</smiles>

According to the general procedure.

Colorless oil (52.4 mg, 83\%).

$R_{\mathrm{f}} 0.75$ (Petroleum ether).

${ }^{1} \mathbf{H}$ NMR $\left(400 \mathrm{MHz}, \mathrm{CDCl}_{3}\right) \delta 7.50-7.38(\mathrm{~m}, 5 \mathrm{H}), 7.29(\mathrm{dd}, J=5.9,2.5 \mathrm{~Hz}, 2 \mathrm{H}), 7.00(\mathrm{~d}, J=8.7$ $\mathrm{Hz}, 2 \mathrm{H}), 5.10$ (s, 2H), $2.36(\mathrm{~s}, 2 \mathrm{H}), 0.86(\mathrm{~s}, 9 \mathrm{H}) .{ }^{13} \mathbf{C} \mathbf{N M R}\left(100 \mathrm{MHz}, \mathrm{CDCl}_{3}\right) \delta 157.7,141.9$, $136.9,129.5\left(\mathrm{t}, J_{\mathrm{C}-\mathrm{F}}=3.1 \mathrm{~Hz}\right), 128.6,128.0,127.6,114.6,90.6\left(\mathrm{dd}, J_{\mathrm{C}-\mathrm{F}}=21.8,13.6 \mathrm{~Hz}\right), 70.0$, 41.2, 32.7, 29.8. ${ }^{19} \mathbf{F}$ NMR $\left(376 \mathrm{MHz}, \mathrm{CDCl}_{3}\right) \delta-90.61(\mathrm{~d}, J=43.2 \mathrm{~Hz}, 1 \mathrm{~F}),-93.17(\mathrm{~d}, J=43.2 \mathrm{~Hz}$, $1 \mathrm{~F})$.

HRMS (ESI) m/z: $[\mathrm{M}+\mathrm{H}]^{+}$Calcd for $\mathrm{C}_{20} \mathrm{H}_{23} \mathrm{~F}_{2} \mathrm{O} 317.1711$; found 317.1711 .

1-(2-(4-(benzyloxy)phenyl)-3,3-difluoroallyl)adamantane (21).<smiles>FC(F)=C(CC12CC3CC(CC(C3)C1)C2)c1ccc(OCc2ccccc2)cc1</smiles>

According to the general procedure.

Colorless oil (69.3mg, $88 \%)$.

$R_{\mathrm{f}} 0.80$ (Petroleum ether).

${ }^{1} \mathbf{H}$ NMR (400 MHz, $\left.\mathrm{CDCl}_{3}\right) \delta 7.38(\mathrm{dt}, J=11.0,4.5 \mathrm{~Hz}, 5 \mathrm{H}), 7.23(\mathrm{dd}, J=6.7,5.4 \mathrm{~Hz}, 2 \mathrm{H}), 6.93$ $(\mathrm{dd}, J=7.4,5.8 \mathrm{~Hz}, 2 \mathrm{H}), 5.03(\mathrm{~d}, J=5.9 \mathrm{~Hz}, 2 \mathrm{H}), 2.15(\mathrm{~s}, 2 \mathrm{H}), 1.85(\mathrm{~s}, 3 \mathrm{H}), 1.62(\mathrm{~d}, J=11.6 \mathrm{~Hz}$, 3H), $1.54(\mathrm{~s}, 4 \mathrm{H}), 1.37(\mathrm{~s}, 6 \mathrm{H}) .{ }^{13} \mathrm{C}$ NMR $\left(100 \mathrm{MHz}, \mathrm{CDCl}_{3}\right) \delta 157.6,136.9,129.4\left(\mathrm{t}, J_{\mathrm{C}-\mathrm{F}}=3.0\right.$ $\mathrm{Hz}), 128.6,128.0,127.6,114.5,89.2\left(\mathrm{dd}, J_{\mathrm{C}-\mathrm{F}}=21.6,12.5 \mathrm{~Hz}\right), 70.0,42.7,41.9,36.9,34.6,28.6$. ${ }^{19} \mathrm{~F} \mathrm{NMR}\left(376 \mathrm{MHz}, \mathrm{CDCl}_{3}\right) \delta-89.88(\mathrm{~d}, J=43.1 \mathrm{~Hz}, 1 \mathrm{~F}),-92.90(\mathrm{~d}, J=43.2 \mathrm{~Hz}, 1 \mathrm{~F})$.

HRMS (EI) m/z: [M] ${ }^{+}$Calcd for $\mathrm{C}_{26} \mathrm{H}_{28} \mathrm{~F}_{2} \mathrm{O}$ 394.2108; found 394.2101.

(3-(4-(benzyloxy)phenyl)-4,4-difluorobut-3-en-1-yl)trimethylsilane (22). 
<smiles>C[Si](C)(C)CCC(=C(F)F)c1ccc(Br)cc1</smiles>

According to the general procedure.

Colorless oil (36.6 mg, 53\%).

$R_{\mathrm{f}} 0.50$ (Petroleum ether).

${ }^{1} \mathbf{H}$ NMR $\left(400 \mathrm{MHz}, \mathrm{CDCl}_{3}\right) \delta{ }^{1} \mathrm{H}$ NMR $\left(400 \mathrm{MHz}, \mathrm{CDCl}_{3}\right) \delta 7.64$ (ddd, $J=17.1,11.4,4.7 \mathrm{~Hz}$, $5 \mathrm{H}), 7.51-7.45(\mathrm{~m}, 2 \mathrm{H}), 7.23(\mathrm{~d}, J=8.8 \mathrm{~Hz}, 2 \mathrm{H}), 5.32(\mathrm{~s}, 2 \mathrm{H}), 2.72-2.48(\mathrm{~m}, 2 \mathrm{H}), 0.93-0.72$ (m, 2H), 0.25 (s, 9H).. ${ }^{13} \mathrm{C}$ NMR $\left(100 \mathrm{MHz}, \mathrm{CDCl}_{3}\right) \delta{ }^{13} \mathrm{C} \mathrm{NMR}\left(100 \mathrm{MHz}, \mathrm{CDCl}_{3}\right) \delta$ 159.7, $138.8,131.3\left(\mathrm{t}, J_{\mathrm{C}-\mathrm{F}}=3.5 \mathrm{~Hz}\right), 130.4,129.8,129.3,116.6,96.1\left(\mathrm{dd}, J_{\mathrm{C}-\mathrm{F}}=20.1,11.3 \mathrm{~Hz}\right), 71.8$, 24.0, 17.1, -0.0. ${ }^{19} \mathbf{F}$ NMR $\left(376 \mathrm{MHz}, \mathrm{CDCl}_{3}\right) \delta-93.19(\mathrm{~d}, J=48.2 \mathrm{~Hz}, 1 \mathrm{~F}),-93.59(\mathrm{~d}, J=48.1 \mathrm{~Hz}$, $1 \mathrm{~F})$.

HRMS (ESI) m/z: [M + Na $]^{+}$Calcd for $\mathrm{C}_{20} \mathrm{H}_{24} \mathrm{~F}_{2} \mathrm{OSiNa}$ 369.1457; found 369.1452 .

(3S,8R,9S,10S,13R,14S,17R)-3-(2-(4-(benzyloxy)phenyl)-3,3-difluoroallyl)-10,13-dimethyl-17 -((R)-6-methylheptan-2-yl)hexadecahydro-1H-cyclopenta[a]phenanthrene (23).

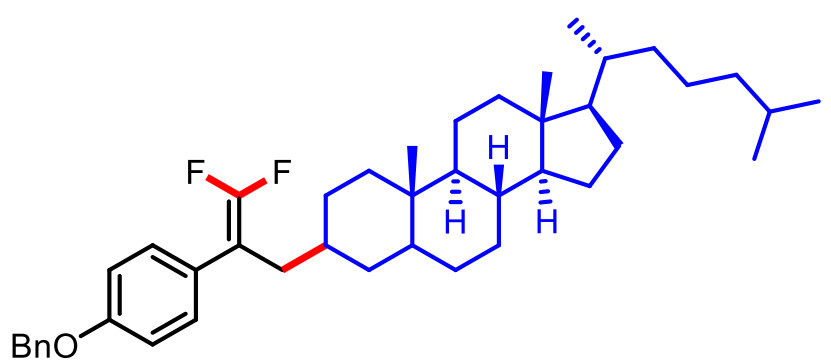

According to the general procedure.

Yellow solid (103.0 mg, 79\%).

$R_{\mathrm{f}} 0.50$ (Petroleum ether).

${ }^{1}$ H NMR $\left(400 \mathrm{MHz}, \mathrm{CDCl}_{3}\right) \delta 7.50-7.30(\mathrm{~m}, 5 \mathrm{H}), 7.20(\mathrm{dd}, J=9.7,7.5 \mathrm{~Hz}, 2 \mathrm{H}), 7.07-6.87(\mathrm{~m}$, 2H), $5.04(\mathrm{~s}, 2 \mathrm{H}), 2.45(\mathrm{qd}, J=14.3,8.0 \mathrm{~Hz}, 1 \mathrm{H}), 2.23(\mathrm{~d}, J=6.6 \mathrm{~Hz}, 1 \mathrm{H}), 2.00-1.91(\mathrm{~m}, 1 \mathrm{H})$, $1.84-1.74(\mathrm{~m}, 1 \mathrm{H}), 1.68-1.59(\mathrm{~m}, 2 \mathrm{H}), 1.56-1.48(\mathrm{~m}, 3 \mathrm{H}), 1.24$ (dddd, $J=37.4,34.7,15.6$, $9.6 \mathrm{~Hz}, 19 \mathrm{H}), 1.07-1.01(\mathrm{~m}, 3 \mathrm{H}), 0.93-0.84(\mathrm{~m}, 11 \mathrm{H}), 0.74(\mathrm{~s}, 3 \mathrm{H}), 0.63(\mathrm{~d}, J=4.4 \mathrm{~Hz}, 3 \mathrm{H})$. ${ }^{13} \mathrm{C}$ NMR $\left(100 \mathrm{MHz}, \mathrm{CDCl}_{3}\right) \delta 157.8,136.9,129.5\left(\mathrm{t}, J_{\mathrm{C}-\mathrm{F}}=3.0 \mathrm{~Hz}\right), 129.4\left(\mathrm{t}, J_{\mathrm{C}-\mathrm{F}}=3.1 \mathrm{~Hz}\right)$, 128.6, 128.0, 127.5, 127.5, 126.2, $91.3\left(\mathrm{dd}, J_{\mathrm{C}-\mathrm{F}}=19.7,14.1 \mathrm{~Hz}\right), 90.4\left(\mathrm{dd}, J_{\mathrm{C}-\mathrm{F}}=21.1,13.1 \mathrm{~Hz}\right)$, 70.0, 56.6, 56.6, 56.3, 56.3, 54.7, 54.6, 46.4, 42.6, 42.6, 40.4, 40.1, 39.5, 38.4, 36.4, 36.2, 36.0, $35.9,35.8,35.5,35.2,33.2,32.3,32.1,30.6,30.2,29.0,28.5,28.3,28.0,24.8,24.2,23.9,23.8$, $22.9,22.6,21.0,20.8,18.7,12.3,12.1,12.1,11.7 .{ }^{19} \mathbf{F} \mathbf{~ N M R}\left(376 \mathrm{MHz}, \mathrm{CDCl}_{3}\right) \delta-91.99--92.81$ (m, 2F).

HRMS (EI) m/z: $[\mathrm{M}+\mathrm{H}]^{+}$Calcd for $\mathrm{C}_{43} \mathrm{H}_{61} \mathrm{~F}_{2} \mathrm{O}$ 631.4690; found 631.4683.

(5S,8R,9S,10S,13S,14S)-3-(2-(4-(benzyloxy)phenyl)-3,3-difluoroallyl)-10,13-dimethylhexadec ahydro-17H-cyclopenta[a]phenanthren-17-one (24) 
<smiles>CC12CC[C@H]3[C@H](CC[C@@H]4CC(CC(=C(F)F)c5ccc(OCc6ccccc6)cc5)CC[C@]34C)[C@@H]1CCC2=O</smiles>

According to the general procedure.

Yellow oil, (86.2 mg).

$\mathrm{R}_{\mathrm{f}} 0.50$ (Petroleum ether/EtOAc, 20/1).

${ }^{1}$ H NMR $\left(400 \mathrm{MHz}, \mathrm{CDCl}_{3}\right) \delta 7.44-7.31(\mathrm{~m}, 5 \mathrm{H}), 7.22(\mathrm{~d}, J=8.6 \mathrm{~Hz}, 2 \mathrm{H}), 6.96(\mathrm{~d}, J=8.8 \mathrm{~Hz}$, 2H), $5.06(\mathrm{~s}, 2 \mathrm{H}), 2.47-2.40(\mathrm{~m}, 2 \mathrm{H}), 2.12-2.03(\mathrm{~m}, 1 \mathrm{H}), 1.97-1.90(\mathrm{~m}, 1 \mathrm{H}), 1.83-1.75(\mathrm{~m}$, 2H), $1.72-1.64(\mathrm{~m}, 2 \mathrm{H}), 1.59-1.37(\mathrm{~m}, 6 \mathrm{H}), 1.33-0.99(\mathrm{~m}, 11 \mathrm{H}), 0.86(\mathrm{~s}, 3 \mathrm{H}), 0.78(\mathrm{~s}, 3 \mathrm{H})$. ${ }^{13} \mathrm{C}$ NMR $\left(100 \mathrm{MHz}, \mathrm{CDCl}_{3}\right) \delta 221.6,157.9,154.0,136.9,129.5\left(\mathrm{t}, J_{\mathrm{C}-\mathrm{F}}=3.0 \mathrm{~Hz}\right), 128.6,128.0$, 127.5, 126.2, 114.7, 91.2, 70.0, 54.8, 51.6, 47.9, 40.5, 36.6, 35.9, 35.1, 33.2, 32.1, 31.6, 30.9, 30.5, 30.1, 28.6, 24.8, 21.8, 20.1, 13.9, 11.7. ${ }^{19} \mathbf{F}$ NMR $\left(376 \mathrm{MHz}, \mathrm{CDCl}_{3}\right) \delta-92.08(\mathrm{~d}, J=46.4 \mathrm{~Hz}, 0.3 \mathrm{~F})$, -92.45 (d, $J=46.4 \mathrm{~Hz}, 0.3 \mathrm{~F}),-92.63$ (s, 1.3F).

HRMS (ESI) m/z: [M + H] $]^{+}$Calcd for $\mathrm{C}_{35} \mathrm{H}_{43} \mathrm{~F}_{2} \mathrm{O}_{2}$ 533.3231; found 533.3224 .

1-((8S,9S,10R,13S,14S,17S)-3-(2-(4-(benzyloxy)phenyl)-3,3-difluoroallyl)-10,13-dimethyl

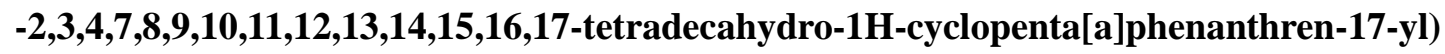
ethan-1-one (25)

(c)

According to the general procedure.

Yellow oil, (91.5 mg, 82\%).

$\mathrm{R}_{\mathrm{f}} 0.5$ (Petroleum ether/EtOAc, 20/1).

${ }^{1} \mathbf{H}$ NMR $\left(400 \mathrm{MHz}, \mathrm{CDCl}_{3}\right) \delta 7.45-7.32(\mathrm{~m}, 5 \mathrm{H}), 7.23(\mathrm{dd}, J=8.6,3.0 \mathrm{~Hz}, 2 \mathrm{H}), 6.96(\mathrm{~d}, J$ $=8.7 \mathrm{~Hz}, 2 \mathrm{H}), 5.24(\mathrm{~d}, J=4.3 \mathrm{~Hz}, 1 \mathrm{H}), 5.06(\mathrm{~s}, 2 \mathrm{H}), 2.58-2.49(\mathrm{~m}, 1 \mathrm{H}), 2.42-2.38(\mathrm{~m}$, $0.7 \mathrm{H}), 2.32-2.27(\mathrm{~m}, 2 \mathrm{H}), 2.24-2.12(\mathrm{~m}, 4 \mathrm{H}), 2.08-1.92(\mathrm{~m}, 3 \mathrm{H}), 1.80-1.58(\mathrm{~m}, 7 \mathrm{H})$, $1.49-1.38(\mathrm{~m}, 4 \mathrm{H}), 1.31-1.18(\mathrm{~m}, 3 \mathrm{H}), 1.14-1.06(\mathrm{~m}, 1 \mathrm{H}), 0.98(\mathrm{~s}, 3 \mathrm{H}), 0.63(\mathrm{~s}, 3 \mathrm{H}) .{ }^{13} \mathrm{C}$ $\operatorname{NMR}\left(100 \mathrm{MHz}, \mathrm{CDCl}_{3}\right) \delta 209.71,209.66,158.6,157.9,153.9\left(\mathrm{dd}, J_{\mathrm{C}-\mathrm{F}}=287.6,284.2 \mathrm{~Hz}\right)$, $153.7\left(\mathrm{dd}, J_{\mathrm{C}-\mathrm{F}}=287.0,284.5 \mathrm{~Hz}\right), 142.7,139.8,136.9,129.5\left(\mathrm{t}, J_{\mathrm{C}-\mathrm{F}}=3.0 \mathrm{~Hz}\right), 129.4\left(\mathrm{t}, J_{\mathrm{C}-\mathrm{F}}\right.$ $=3.1 \mathrm{~Hz}), 128.6,128.0,127.53,127.51,126.4,126.2,121.5,119.4,114.8,114.7,91.0(\mathrm{dd}$, $\left.J_{\mathrm{C}-\mathrm{F}}=22.1,13.1 \mathrm{~Hz}\right), 90.3\left(\mathrm{dd}, J_{\mathrm{C}-\mathrm{F}}=21.6,13.2 \mathrm{~Hz}\right), 70.0,63.8,63.7,57.0,50.4,50.2,44.05$, 44.02, 39.3, 39.1, 38.91, 38.89, 37.4, 37.1, 36.4, 35.1, 34.1, 32.0, 31.88, 31.85, 31.7, 31.60, $31.58,29.1,28.6,25.4,24.5,22.84,22.81,20.9,20.8,19.5,19.4,13.3 .{ }^{19} \mathbf{F}$ NMR $(376 \mathrm{MHz}$, $\left.\mathrm{CDCl}_{3}\right) \delta-91.86--92.68(\mathrm{~m}, 2 \mathrm{~F})$. 
HRMS (ESI) m/z: $[\mathrm{M}+\mathrm{H}]^{+}$Calcd for $\mathrm{C}_{37} \mathrm{H}_{45} \mathrm{~F}_{2} \mathrm{O}_{2}$ 559.3388; found 559.3381.

tert-butyl 4-(2-([1,1'-biphenyl]-4-yl)-3,3-difluoroallyl)piperidine-1-carboxylate (26).<smiles>CC(C)(C)OC(=O)N1CCC(CC(=C(F)F)c2ccc(-c3ccccc3)cc2)CC1</smiles>

According to the general procedure.

White solid (39.6 mg, 48\%). M.p. $=141-142{ }^{\circ} \mathrm{C}$.

$R_{\mathrm{f}} 0.50$ (Petroleum ether/EtOAc, 5/1).

${ }^{1} \mathbf{H}$ NMR $\left(400 \mathrm{MHz}, \mathrm{CDCl}_{3}\right) \delta 7.66-7.58(\mathrm{~m}, 4 \mathrm{H}), 7.51-7.44(\mathrm{~m}, 2 \mathrm{H}), 7.44-7.36(\mathrm{~m}, 3 \mathrm{H}), 4.08$ (s, 2H), $2.62(\mathrm{~s}, 2 \mathrm{H}), 2.41(\mathrm{~d}, J=4.9 \mathrm{~Hz}, 2 \mathrm{H}), 1.68(\mathrm{~d}, J=12.7 \mathrm{~Hz}, 2 \mathrm{H}), 1.47(\mathrm{~d}, J=3.1 \mathrm{~Hz}, 9 \mathrm{H})$, $1.30(\mathrm{~d}, J=9.1 \mathrm{~Hz}, 1 \mathrm{H}), 1.17(\mathrm{~d}, J=11.7 \mathrm{~Hz}, 2 \mathrm{H}) .{ }^{13} \mathbf{C}$ NMR $\left(100 \mathrm{MHz}, \mathrm{CDCl}_{3}\right) \delta{ }^{13} \mathrm{C}$ NMR $(100$ $\left.\mathrm{MHz}, \mathrm{CDCl}_{3}\right) \delta 154.8,140.4,140.1,132.5,128.8,128.5\left(\mathrm{t}, J_{\mathrm{C}-\mathrm{F}}=3.2 \mathrm{~Hz}\right), 127.4,127.2,127.0$, $90.1\left(\mathrm{dd}, J_{\mathrm{C}-\mathrm{F}}=22.0,13.0 \mathrm{~Hz}\right), 79.3,44.1,34.2,31.7,28.4 .{ }^{19} \mathbf{F} \mathbf{~ N M R}\left(376 \mathrm{MHz}, \mathrm{CDCl}_{3}\right) \delta$ $-90.05(\mathrm{~d}, J=41.7 \mathrm{~Hz}, 1 \mathrm{~F}),-90.53(\mathrm{~d}, J=41.9 \mathrm{~Hz}, 1 \mathrm{~F})$.

HRMS (ESI) m/z: [M + Na] $]^{+}$Calcd for $\mathrm{C}_{25} \mathrm{H}_{29} \mathrm{~F}_{2} \mathrm{NNaO}_{2} 436.2059$; found 436.2055.

tert-butyl 4-(2-(3-(benzyloxy)phenyl)-3,3-difluoroallyl)piperidine-1-carboxylate (27).<smiles>CC(C)(C)OC(=O)N1CCC(CC(=C(F)F)c2cccc(OCc3ccccc3)c2)CC1</smiles>

According to the general procedure.

Pale oil (39.9 mg, $45 \%)$.

$R_{\mathrm{f}} 0.50$ (Petroleum ether/EtOAc, 5/1).

${ }^{1} \mathbf{H}$ NMR $\left(400 \mathrm{MHz}, \mathrm{CDCl}_{3}\right) \delta 11.90(\mathrm{~s}, 1 \mathrm{H}), 8.33(\mathrm{~d}, J=7.6 \mathrm{~Hz}, 1 \mathrm{H}), 7.78(\mathrm{ddd}, J=12.4,9.6,4.4$ $\mathrm{Hz}, 2 \mathrm{H}), 7.59-7.45(\mathrm{~m}, 1 \mathrm{H}), 7.22(\mathrm{~d}, J=8.0 \mathrm{~Hz}, 2 \mathrm{H}), 7.11(\mathrm{~d}, J=8.0 \mathrm{~Hz}, 2 \mathrm{H}), 3.25(\mathrm{dd}, J=13.2$, $6.4 \mathrm{~Hz}, 1 \mathrm{H}), 3.17(\mathrm{dd}, J=14.8,6.8 \mathrm{~Hz}, 1 \mathrm{H}), 2.87(\mathrm{ddd}, J=20.8,13.6,7.6 \mathrm{~Hz}, 2 \mathrm{H}), 1.41(\mathrm{~d}, J=$ $6.8 \mathrm{~Hz}, 3 \mathrm{H}), 1.20(\mathrm{dd}, J=6.8,2.0 \mathrm{~Hz}, 6 \mathrm{H}) .{ }^{13} \mathbf{C} \mathbf{~ N M R}\left(100 \mathrm{MHz}, \mathrm{CDCl}_{3}\right) \delta 158.7,154.7,136.7$, 135.0, 129.5, 128.6, 128.1, 127.5, $120.9\left(\mathrm{t}, J_{\mathrm{C}-\mathrm{F}}=3.1 \mathrm{~Hz}\right), 115.2,113.4,90.4\left(\mathrm{dd}, J_{\mathrm{C}-\mathrm{F}}=19.4\right.$, $15.8 \mathrm{~Hz}), 79.3,70.0,43.6,34.3,34.1,31.6,28.4 .{ }^{19} \mathbf{F ~ N M R}\left(376 \mathrm{MHz}, \mathrm{CDCl}_{3}\right) \delta-90.36(\mathrm{~s}, 2 \mathrm{~F})$.

HRMS (ESI) m/z: [M + Na] $]^{+}$Calcd for $\mathrm{C}_{26} \mathrm{H}_{31} \mathrm{~F}_{2} \mathrm{NNaO}_{3} 466.2164$; found 466.2159.

tert-butyl 4-(2-(3-chlorophenyl)-3,3-difluoroallyl)piperidine-1-carboxylate (28). 
<smiles>CC(C)(C)OC(=O)N1CCC(CC(=C(F)F)c2cccc(Cl)c2)CC1</smiles>

According to the general procedure.

White solid (48.2 mg, 65\%). M.p. $=148-149{ }^{\circ} \mathrm{C}$.

$R_{\mathrm{f}} 0.50$ (Petroleum ether/EtOAc, 5/1).

${ }^{1} \mathbf{H}$ NMR $\left(400 \mathrm{MHz}, \mathrm{CDCl}_{3}\right) \delta 7.32(\mathrm{dd}, J=12.0,9.4 \mathrm{~Hz}, 4 \mathrm{H}), 4.07(\mathrm{~s}, 2 \mathrm{H}), 2.62-2.60(\mathrm{~m}, 2 \mathrm{H})$, $2.35-2.34(\mathrm{~m}, 2 \mathrm{H}), 1.63-1.62(\mathrm{~m}, 2 \mathrm{H}), 1.47-1.43(\mathrm{~m}, 10 \mathrm{H}), 1.20-1.06(\mathrm{~m}, 2 \mathrm{H}) .{ }^{13} \mathbf{C}$ NMR $\left(100 \mathrm{MHz}, \mathrm{CDCl}_{3}\right) \delta 154.7,154.6154 .2(\mathrm{dd}, J=290.1,284.2 \mathrm{~Hz}), 135.5,134.4,129.8,128.2(\mathrm{t}, J=$ $3.4 \mathrm{~Hz}), 127.5,126.4\left(\mathrm{t}, J_{\mathrm{C}-\mathrm{F}}=3.2 \mathrm{~Hz}\right), 89.8(\mathrm{dd}, J=22.8,12.9 \mathrm{~Hz}), 79.8,79.3,43.3,37.3,34.2$, 31.6, 28.4, 28.4, 27.7. ${ }^{19} \mathbf{F}$ NMR $\left(376 \mathrm{MHz}, \mathrm{CDCl}_{3}\right) \delta-89.31(\mathrm{~d}, J=39.5 \mathrm{~Hz}, 1 \mathrm{~F}),-89.71(\mathrm{~d}, J=$ $39.5 \mathrm{~Hz}, 1 \mathrm{~F})$.

HRMS (ESI) m/z: [M + Na $]^{+}$Calcd for $\mathrm{C}_{19} \mathrm{H}_{24} \mathrm{ClF}_{2} \mathrm{NNaO}_{2}$ 394.1356; found 394.1352.

tert-butyl 4-(2-(3,4-dichlorophenyl)-3,3-difluoroallyl)piperidine-1-carboxylate (29).<smiles>CC(C)(C)OC(=O)N1CCC(CC(=C(F)F)c2ccc(Cl)c(Cl)c2)CC1</smiles>

According to the general procedure.

White solid (57.5 mg, 71\%). M.p. $=149-150{ }^{\circ} \mathrm{C}$.

$R_{\mathrm{f}} 0.40$ (Petroleum ether/EtOAc, 5/1).

${ }^{1}$ H NMR $\left(400 \mathrm{MHz}, \mathrm{CDCl}_{3}\right) \delta 7.50-7.34(\mathrm{~m}, 2 \mathrm{H}), 7.12(\mathrm{~d}, J=8.2 \mathrm{~Hz}, 1 \mathrm{H}), 4.03(\mathrm{~s}, 2 \mathrm{H}), 2.56(\mathrm{~s}$, 2H), $2.28(\mathrm{~d}, J=6.2 \mathrm{~Hz}, 2 \mathrm{H}), 1.59(\mathrm{~s}, 3 \mathrm{H}), 1.42(\mathrm{~s}, 9 \mathrm{H}), 1.09(\mathrm{~d}, J=9.9 \mathrm{~Hz}, 2 \mathrm{H}) .{ }^{13} \mathbf{C}$ NMR $(100$ $\left.\mathrm{MHz}, \mathrm{CDCl}_{3}\right) \delta 154.7,133.6,132.7,131.4,130.5,130.0(\mathrm{t}, J=3.2 \mathrm{~Hz}), 127.5(\mathrm{t}, J=3.4 \mathrm{~Hz})$, 89.2(dd, $J=22.8,12.6 \mathrm{~Hz}), 79.4,43.9,37.3,34.1,31.6,28.4,27.7 .{ }^{19} \mathbf{F} \mathbf{N M R}\left(376 \mathrm{MHz}, \mathrm{CDCl}_{3}\right) \delta$ $-88.50(\mathrm{~d}, J=38.3 \mathrm{~Hz}, 1 \mathrm{~F}),-88.98(\mathrm{~d}, J=38.2 \mathrm{~Hz}, 1 \mathrm{~F})$.

HRMS (ESI) m/z: [M + Na] ${ }^{+}$Calcd for $\mathrm{C}_{19} \mathrm{H}_{23} \mathrm{Cl}_{2} \mathrm{~F}_{2} \mathrm{NNaO}_{2}$ 428.0966; found 428.0965 .

1-(benzyloxy)-3-(3-cyclohexyl-1,1-difluoroprop-1-en-2-yl)benzene (30).<smiles>FC(F)=C(CC1CCCCC1)c1cccc(Cc2ccccc2)c1</smiles>

According to the general procedure.

Yellow oil (50.0 mg, 73\%).

$R_{\mathrm{f}} 0.80$ (Petroleum ether).

${ }^{1} \mathbf{H}$ NMR $\left(400 \mathrm{MHz}, \mathrm{CDCl}_{3}\right) \delta 7.53-7.35(\mathrm{~m}, 5 \mathrm{H}), 7.34-7.29(\mathrm{~m}, 1 \mathrm{H}), 7.01-6.89(\mathrm{~m}, 3 \mathrm{H}), 5.11$ $(\mathrm{d}, J=2.0 \mathrm{~Hz}, 2 \mathrm{H}), 2.46-2.19(\mathrm{~m}, 2 \mathrm{H}), 1.66(\mathrm{dd}, J=33.4,15.0 \mathrm{~Hz}, 5 \mathrm{H}), 1.28(\mathrm{dd}, J=10.4,7.8$ $\mathrm{Hz}, 1 \mathrm{H}), 1.16$ (s, 3H), $1.02-0.85(\mathrm{~m}, 2 \mathrm{H}) .{ }^{13} \mathrm{C}$ NMR $\left(100 \mathrm{MHz}, \mathrm{CDCl}_{3}\right) \delta{ }^{13} \mathrm{C} \mathrm{NMR}(100 \mathrm{MHz}$, 
$\left.\mathrm{CDCl}_{3}\right) \delta 158.7,136.9,129.3(\mathrm{t}, J=3.2 \mathrm{~Hz}), 128.6,128.0,127.6,121.0,115.2,113.2,91.0(\mathrm{dd}, J=$ 20.2, 15.0 Hz), 70.0, 35.6, 35.2, 32.8, 26.4, 26.0. ${ }^{19} \mathbf{F}$ NMR (376 MHz, $\left.\mathrm{CDCl}_{3}\right) \delta-90.92(\mathrm{~d}, 1 \mathrm{~F})$, $-91.16(\mathrm{~d}, 1 \mathrm{~F})$.

HRMS (ESI) m/z: [M + H] $]^{+}$Calcd for $\mathrm{C}_{22} \mathrm{H}_{25} \mathrm{~F}_{2} \mathrm{O}$ 343.1868; found 343.1865.

5-(3-cyclohexyl-1,1-difluoroprop-1-en-2-yl)-1,2,3-trimethoxybenzene (31).<smiles>COc1cc(C(CC2CCCCC2)=C(F)F)cc(OC)c1OC</smiles>

According to the general procedure.

Yellow solid (56.1 mg, 86\%); M.p. $=70-71{ }^{\circ} \mathrm{C}$.

$R_{\mathrm{f}} 0.6$ (Petroleum ether/EtOAc, 3/1).

${ }^{1} \mathbf{H}$ NMR (400 MHz, $\left.\mathrm{CDCl}_{3}\right) \delta 6.48(\mathrm{~s}, 2 \mathrm{H}), 3.84(\mathrm{~s}, 3 \mathrm{H}), 3.83(\mathrm{~s}, 6 \mathrm{H}), 2.27-2.19(\mathrm{~m}, 2 \mathrm{H}), 1.66$ $(\mathrm{d}, J=10.2 \mathrm{~Hz}, 5 \mathrm{H}), 1.31-1.22(\mathrm{~m}, 1 \mathrm{H}), 1.11(\mathrm{~d}, J=8.1 \mathrm{~Hz}, 3 \mathrm{H}), 0.92(\mathrm{t}, J=10.9 \mathrm{~Hz}, 2 \mathrm{H}) .{ }^{13} \mathbf{C}$ NMR $\left(100 \mathrm{MHz}, \mathrm{CDCl}_{3}\right) \delta{ }^{13} \mathrm{C} \mathrm{NMR}\left(100 \mathrm{MHz}, \mathrm{CDCl}_{3}\right) \delta 153.8,153.0,137.2,129.6,105.7,91.1$ $(\mathrm{dd}, J=22.0,12.4 \mathrm{~Hz}), 60.8,56.1,35.7,35.5,32.9,26.4,26.0 .{ }^{19} \mathbf{F} \mathbf{N M R}\left(376 \mathrm{MHz}, \mathrm{CDCl}_{3}\right) \delta$ $-91.13(\mathrm{~d}, J=44.6 \mathrm{~Hz}, 1 \mathrm{~F}),-91.50(\mathrm{~d}, J=44.7 \mathrm{~Hz}, 1 \mathrm{~F})$.

HRMS (ESI) m/z: [M + H] $]^{+}$Calcd for $\mathrm{C}_{18} \mathrm{H}_{25} \mathrm{~F}_{2} \mathrm{O}_{3}$ 327.1766; found 327.1760.

\section{2-(3-cyclohexyl-1,1-difluoroprop-1-en-2-yl)naphthalene (32).}<smiles>FC(F)=C(CC1CCCCC1)c1ccc2ccccc2c1</smiles>

According to the general procedure.

Yellow oil (49.0 mg, 85\%).

$R_{\mathrm{f}} 0.80$ (Petroleum ether).

${ }^{1} \mathbf{H}$ NMR $\left(400 \mathrm{MHz}, \mathrm{CDCl}_{3}\right) \delta 8.00-7.77(\mathrm{~m}, 4 \mathrm{H}), 7.57-7.45(\mathrm{~m}, 3 \mathrm{H}), 2.43(\mathrm{dd}, J=5.0,2.1 \mathrm{~Hz}$, $2 \mathrm{H}), 1.83-1.55(\mathrm{~m}, 5 \mathrm{H}), 1.40-1.26(\mathrm{~m}, 1 \mathrm{H}), 1.23-1.09(\mathrm{~m}, 3 \mathrm{H}), 1.00(\mathrm{dd}, J=22.7,11.3 \mathrm{~Hz}$, 2H). ${ }^{13} \mathrm{C}$ NMR $\left(100 \mathrm{MHz}, \mathrm{CDCl}_{3}\right) \delta{ }^{13} \mathrm{C} \mathrm{NMR}\left(100 \mathrm{MHz}, \mathrm{CDCl}_{3}\right) \delta 154.2\left(\mathrm{dd}, J_{\mathrm{C}-\mathrm{F}}=288.6\right.$, $284.6 \mathrm{~Hz}), 133.3,132.4,131.6\left(\mathrm{t}, J_{\mathrm{C}-\mathrm{F}}=3.8 \mathrm{~Hz}\right), 128.0,127.9,127.6,127.3,126.3,126.2$, 126.0, $91.2\left(\mathrm{dd}, J_{\mathrm{C}-\mathrm{F}}=22.3,12.5 \mathrm{~Hz}\right), 35.7,35.3,32.9,26.4,26.0 .{ }^{19} \mathbf{F} \mathbf{~ N M R}\left(376 \mathrm{MHz}, \mathrm{CDCl}_{3}\right)$ $\delta-90.86(\mathrm{~d}, J=43.2 \mathrm{~Hz}, 1 \mathrm{~F}),-91.56(\mathrm{~d}, J=43.3 \mathrm{~Hz}, 1 \mathrm{~F})$.

HRMS (EI) m/z: [M] $]^{+}$Calcd for $\mathrm{C}_{19} \mathrm{H}_{20} \mathrm{~F}_{2}$ 286.1533; found 286.1527 .

\section{4-(3-cyclohexyl-1,1-difluoroprop-1-en-2-yl)-1,1'-biphenyl (33).}


<smiles>FC(F)=C(CC1CCCCC1)c1ccc(-c2ccccc2)cc1</smiles>

According to the general procedure.

White solid (51.8 mg, 83\%). M.p. $=105-106{ }^{\circ} \mathrm{C}$.

$R_{\mathrm{f}} 0.60$ (Petroleum ether).

${ }^{1} \mathbf{H}$ NMR $\left(400 \mathrm{MHz}, \mathrm{CDCl}_{3}\right) \delta 7.60(\mathrm{dd}, J=13.4,5.0 \mathrm{~Hz}, 4 \mathrm{H}), 7.47-7.31(\mathrm{~m}, 5 \mathrm{H}), 2.34-2.26(\mathrm{~m}$, 2H), $1.79-1.57(\mathrm{~m}, 5 \mathrm{H}), 1.37-1.23(\mathrm{~m}, 1 \mathrm{H}), 1.12(\mathrm{~d}, J=5.3 \mathrm{~Hz}, 3 \mathrm{H}), 0.93(\mathrm{dd}, J=21.6,10.4$ $\mathrm{Hz}, 2 \mathrm{H}) .{ }^{13} \mathbf{C}$ NMR $\left(100 \mathrm{MHz}, \mathrm{CDCl}_{3}\right) \delta 154.1\left(\mathrm{dd}, J_{\mathrm{C}-\mathrm{F}}=288.6,284.6 \mathrm{~Hz}\right), 140.6,139.8,133.1$, 133.0, 133.0, $128.8\left(\mathrm{t}, J_{\mathrm{C}-\mathrm{F}}=3.8 \mathrm{~Hz}\right), 128.6,128.6,128.6,127.3,127.0,127.0,126.9,90.9(\mathrm{dd}$, $\left.J_{\mathrm{C}-\mathrm{F}}=22.3,12.5 \mathrm{~Hz}\right), 35.7,35.1,32.9,26.4,26.1 .{ }^{19} \mathbf{F} \mathbf{~ N M R}\left(376 \mathrm{MHz}, \mathrm{CDCl}_{3}\right) \delta-90.65(\mathrm{~d}, J=$ $43.2 \mathrm{~Hz}, 1 \mathrm{~F}),-91.22(\mathrm{~d}, J=43.4 \mathrm{~Hz}, 1 \mathrm{~F})$.

HRMS (EI) m/z: [M] ${ }^{+}$Calcd for $\mathrm{C}_{21} \mathrm{H}_{22} \mathrm{~F}_{2}$ 312.1690; found 312.1683.

4-bromo-4'-(3-cyclohexyl-1,1-difluoroprop-1-en-2-yl)-1,1'-biphenyl (34).<smiles>FC(F)=C(CC1CCCCC1)c1ccc(-c2ccc(Br)cc2)cc1</smiles>

According to the general procedure.

Yellow oil (64.0 mg, 82\%).

$R_{\mathrm{f}} 0.70$ (Petroleum ether).

${ }^{1}$ H NMR $\left(400 \mathrm{MHz}, \mathrm{CDCl}_{3}\right) \delta 7.54(\mathrm{dd}, J=12.3,5.4 \mathrm{~Hz}, 4 \mathrm{H}), 7.48-7.42(\mathrm{~m}, 2 \mathrm{H}), 7.37(\mathrm{~d}, J=$ $7.4 \mathrm{~Hz}, 2 \mathrm{H}), 2.33-2.25(\mathrm{~m}, 2 \mathrm{H}), 1.65(\mathrm{dd}, J=27.1,15.9 \mathrm{~Hz}, 5 \mathrm{H}), 1.33-1.25(\mathrm{~m}, 1 \mathrm{H}), 1.12(\mathrm{~s}$, 2H), $0.92(\mathrm{dd}, J=21.2,10.7 \mathrm{~Hz}, 2 \mathrm{H}) .{ }^{13} \mathrm{C}$ NMR $\left(100 \mathrm{MHz}, \mathrm{CDCl}_{3}\right) \delta{ }^{13} \mathrm{C} \mathrm{NMR}(100 \mathrm{MHz}$, $\left.\mathrm{CDCl}_{3}\right) \delta 154.0\left(\mathrm{dd}, J_{\mathrm{C}-\mathrm{F}}=287.9,285.6 \mathrm{~Hz}\right), 139.5,138.6,131.9,128.7\left(\mathrm{t}, J_{\mathrm{C}-\mathrm{F}}=3.8 \mathrm{~Hz}\right), 128.5$, $126.8,121.6,90.7\left(\mathrm{dd}, J_{\mathrm{C}-\mathrm{F}}=22.3,12.5 \mathrm{~Hz}\right), 35.7,35.0,32.8,26.4,26.0 .{ }^{19} \mathbf{F}$ NMR $(376 \mathrm{MHz}$, $\left.\mathrm{CDCl}_{3}\right) \delta-90.65(\mathrm{~d}, J=43.2 \mathrm{~Hz}, 1 \mathrm{~F}),-91.22(\mathrm{~d}, J=43.4 \mathrm{~Hz}, 1 \mathrm{~F})$.

HRMS (ESI) m/z: [M + H] $]^{+}$Calcd for $\mathrm{C}_{21} \mathrm{H}_{22} \mathrm{BrF}_{2}$ 391.0867; found 391.0862 .

methyl 4-(3-cyclohexyl-1,1-difluoroprop-1-en-2-yl)benzoate (35).<smiles>COC(=O)c1ccc(C(CC2CCCCC2)=C(F)F)cc1</smiles>

According to the general procedure.

Yellow oil (31.1 mg, 53\%).

$R_{\mathrm{f}} 0.65$ (Petroleum ether/EtOAc, 20/1). 
${ }^{1}$ H NMR $\left(400 \mathrm{MHz}, \mathrm{CDCl}_{3}\right) \delta 8.05(\mathrm{t}, J=6.9 \mathrm{~Hz}, 2 \mathrm{H}), 7.41(\mathrm{t}, J=8.4 \mathrm{~Hz}, 2 \mathrm{H}), 3.95(\mathrm{~s}, 3 \mathrm{H}), 2.53$ $-2.19(\mathrm{~m}, 2 \mathrm{H}), 1.66(\mathrm{t}, J=13.7 \mathrm{~Hz}, 5 \mathrm{H}), 1.26(\mathrm{dd}, J=14.0,6.5 \mathrm{~Hz}, 1 \mathrm{H}), 1.13(\mathrm{~s}, 2 \mathrm{H}), 0.98-0.84$ $(\mathrm{m}, 2 \mathrm{H}) .{ }^{13} \mathrm{C}$ NMR $\left(100 \mathrm{MHz}, \mathrm{CDCl}_{3}\right) \delta 166.8,154.1\left(\mathrm{dd}, J_{\mathrm{C}-\mathrm{F}}=288.9,286.6 \mathrm{~Hz}\right), 129.8\left(\mathrm{t}, J_{\mathrm{C}-\mathrm{F}}=\right.$ $3.8 \mathrm{~Hz}), 129.6,129.1,128.7,128.2,90.9\left(\mathrm{dd}, J_{\mathrm{C}-\mathrm{F}}=22.3,12.5 \mathrm{~Hz}\right), 52.1,35.8,34.8,34.1,32.8$, 26.3, 26.0. ${ }^{19} \mathbf{F}$ NMR (376 MHz, $\left.\mathrm{CDCl}_{3}\right) \delta-89.01(\mathrm{~d}, J=39.0 \mathrm{~Hz}, 1 \mathrm{~F}),-89.69(\mathrm{~d}, J=39.1 \mathrm{~Hz}, 1 \mathrm{~F})$. HRMS (ESI) m/z: [M + H] $]^{+}$Calcd for $\mathrm{C}_{17} \mathrm{H}_{21} \mathrm{~F}_{2} \mathrm{O}_{2}$ 295.1510; found 295.1503.

(E)-(2-cyclohexylvinyl)benzene (36).<smiles>S=CC1CCCCC1/C=C/c1ccccc1</smiles>

According to the general procedure.

Yellow oil (22.3 mg, 60\%).

$R_{\mathrm{f}} 0.70$ (Petroleum ether).

${ }^{1} \mathbf{H}$ NMR $\left(400 \mathrm{MHz}, \mathrm{CDCl}_{3}\right) \delta 7.25(\mathrm{ddt}, J=30.1,23.8,7.4 \mathrm{~Hz}, 5 \mathrm{H}), 6.32(\mathrm{t}, J=13.1 \mathrm{~Hz}, 1 \mathrm{H})$, $6.17(\mathrm{dd}, J=16.0,6.9 \mathrm{~Hz}, 0.62 \mathrm{H}), 5.53-5.43(\mathrm{~m}, 0.39 \mathrm{H}), 2.58(\mathrm{q}, J=10.5 \mathrm{~Hz}, 0.40 \mathrm{H}), 2.25-$ $2.03(\mathrm{~m}, 0.66 \mathrm{H}), 1.89-1.62(\mathrm{~m}, 5 \mathrm{H}), 1.36-1.13(\mathrm{~m}, 5 \mathrm{H}) .{ }^{13} \mathbf{C} \mathbf{~ N M R}\left(100 \mathrm{MHz}, \mathrm{CDCl}_{3}\right) \delta 139.0$, 138.0, 136.8, 128.6, 128.5, 128.2, 127.2, 126.8, 126.7, 126.4, 125.9, 41.2, 36.9, 33.3, 33.0, 26.2, 26.1, 25.7 .

HRMS (EI) m/z: [M] ${ }^{+}$Calcd for $\mathrm{C}_{14} \mathrm{H}_{18}[\mathrm{M}]^{+}$186.1409, found 186.1402 .

methyl (E)-4-(2-cyclohexylvinyl)benzoate (37).<smiles>COC(=O)c1ccc(/C=C/C2CCCCC2)cc1</smiles>

According to the general procedure.

Yellow oil (25.4 mg, 52\%).

$R_{\mathrm{f}} 0.50$ (Petroleum ether/EtOAc, 20/1).

${ }^{1} \mathbf{H}$ NMR $\left(400 \mathrm{MHz}, \mathrm{CDCl}_{3}\right) \delta 7.97(\mathrm{dd}, J=17.5,8.2 \mathrm{~Hz}, 2 \mathrm{H}), 7.35(\mathrm{dd}, J=32.2,8.2 \mathrm{~Hz}, 2 \mathrm{H})$, $6.52-6.19(\mathrm{~m}, 1.50 \mathrm{H}), 5.69-5.49(\mathrm{~m}, 0.51 \mathrm{H}), 3.91(\mathrm{~d}, J=5.4 \mathrm{~Hz}, 3 \mathrm{H}), 2.54(\mathrm{~d}, J=10.5 \mathrm{~Hz}$, $0.52 \mathrm{H}), 2.20-2.11(\mathrm{~m}, 0.54 \mathrm{H}), 1.85-1.65(\mathrm{~m}, 5 \mathrm{H}), 1.24(\mathrm{ddd}, J=25.7,13.1,8.7 \mathrm{~Hz}, 5 \mathrm{H}) .{ }^{13} \mathrm{C}$ NMR $\left(100 \mathrm{MHz}, \mathrm{CDCl}_{3}\right) \delta{ }^{13} \mathrm{C}$ NMR $\left(100 \mathrm{MHz}, \mathrm{CDCl}_{3}\right) \delta 167.0,142.7,142.6,141.0,139.7$, $129.8,129.5,128.5,128.1,128.0,126.5,126.1,125.8,52.0,52.0,41.2,37.1,33.1,32.7,26.1,25.9$, 25.9, 25.6 .

HRMS (ESI) $\mathrm{m} / \mathrm{z}$ : $[\mathrm{M}+\mathrm{H}]^{+}$Calcd for $\mathrm{C}_{16} \mathrm{H}_{21} \mathrm{O}_{2}$ 245.1536; found 245.1538 .

(E)-4-(2-cyclohexylvinyl)phenyl acetate (38). 
<smiles>CC(=O)Oc1ccc(/C=C/C2CCCCC2)cc1</smiles>

$\mathrm{Z} / \mathrm{E}=9: 1$

According to the general procedure.

Yellow oil (23.0 mg, 47\%).

$R_{\mathrm{f}} 0.60$ (Petroleum ether/EtOAc, 20/1).

${ }^{1} \mathbf{H}$ NMR $\left(400 \mathrm{MHz}, \mathrm{CDCl}_{3}\right) \delta 7.37(\mathrm{~d}, J=8.5 \mathrm{~Hz}, 2 \mathrm{H}), 7.05(\mathrm{t}, J=7.6 \mathrm{~Hz}, 2 \mathrm{H}), 6.33$ (t, $J=16.0$ $\mathrm{Hz}, 1 \mathrm{H}), 6.16(\mathrm{dd}, J=15.9,6.9 \mathrm{~Hz}, 0.91 \mathrm{H}), 5.52(\mathrm{t}, J=10.9 \mathrm{~Hz}, 0.09 \mathrm{H}), 2.33(\mathrm{~d}, J=7.5 \mathrm{~Hz}, 3 \mathrm{H})$, $2.15(\mathrm{~d}, J=6.9 \mathrm{~Hz}, 1 \mathrm{H}), 1.89-1.68(\mathrm{~m}, 5 \mathrm{H}), 1.39-1.17(\mathrm{~m}, 5 \mathrm{H}) .{ }^{13} \mathbf{C} \mathbf{~ N M R}\left(100 \mathrm{MHz}, \mathrm{CDCl}_{3}\right) \delta$ 169.6, 149.4, 137.1, 135.9, 126.8, 126.2, 121.5, 41.1, 32.9, 26.1, 26.0, 21.1.

HRMS (ESI) m/z: [M + H] ${ }^{+}$Calcd for $\mathrm{C}_{16} \mathrm{H}_{21} \mathrm{O}_{2}$ 245.1536; found 245.1537.

(E)-1-bromo-4-(2-cyclohexylvinyl)benzene (39).<smiles>Brc1ccc(/C=C/C2CCCCC2)cc1</smiles>

According to the general procedure.

Colorless oil (32.2 mg, 61\%).

$\mathrm{R}_{\mathrm{f}} 0.80$ (Petroleum ether).

${ }^{1}$ H NMR $\left(400 \mathrm{MHz}, \mathrm{CDCl}_{3}\right) \delta 7.45(\mathrm{dd}, J=19.1,7.0 \mathrm{~Hz}, 2 \mathrm{H}), 7.31-7.11(\mathrm{~m}, 2 \mathrm{H}), 6.41-6.13$ $(\mathrm{m}, 1.40 \mathrm{H}), 5.55(\mathrm{t}, J=10.9 \mathrm{~Hz}, 0.63 \mathrm{H}), 2.53(\mathrm{~d}, J=10.3 \mathrm{~Hz}, 0.66 \mathrm{H}), 2.15(\mathrm{~s}, 0.42 \mathrm{H}), 1.79(\mathrm{dd}, J$ $=32.9,7.8 \mathrm{~Hz}, 5 \mathrm{H}), 1.36-1.17(\mathrm{~m}, 5 \mathrm{H}) .{ }^{13} \mathbf{C} \mathbf{~ N M R}\left(100 \mathrm{MHz}, \mathrm{CDCl}_{3}\right) \delta 139.7,137.7,136.8$, 131.5, 131.2, 130.2, 127.5, 126.1, 125.7, 120.2, 41.1, 36.9, 33.1, 32.8, 26.1, 26.0, 25.9, 25.6.

HRMS (ESI) m/z: [M] ${ }^{+}$Calcd for $\mathrm{C}_{14} \mathrm{H}_{17} \mathrm{Br} 264.0514$; found 264.0507 .

3-(4'-(3-cyclohexyl-1,1-difluoroprop-1-en-2-yl)-[1,1'-biphenyl]-4-yl)pyridine (41).<smiles>FC(F)=C(CCCl)c1ccc(-c2ccc(-c3cccnc3)cc2)cc1</smiles>

According to the general procedure.

Yellow oil (58.4 mg, 75\%).

$R_{\mathrm{f}} 0.50$ (Petroleum ether/EtOAc, 2/1).

${ }^{1} \mathbf{H}$ NMR $\left(400 \mathrm{MHz}, \mathrm{CDCl}_{3}\right) \delta 8.93(\mathrm{~s}, 1 \mathrm{H}), 8.63(\mathrm{~d}, J=4.7 \mathrm{~Hz}, 1 \mathrm{H}), 7.96(\mathrm{~d}, J=7.9 \mathrm{~Hz}, 1 \mathrm{H})$, $7.79-7.61(\mathrm{~m}, 6 \mathrm{H}), 7.49-7.38(\mathrm{~m}, 3 \mathrm{H}), 2.35(\mathrm{~d}, J=7.1 \mathrm{~Hz}, 2 \mathrm{H}), 1.76(\mathrm{dd}, J=35.9,22.4 \mathrm{~Hz}$, $5 \mathrm{H}), 1.31(\mathrm{dd}, J=14.4,10.1 \mathrm{~Hz}, 2 \mathrm{H}), 1.16-1.14(\mathrm{~m}, 1 \mathrm{H}), 1.05-0.91(\mathrm{~m}, 2 \mathrm{H}) .{ }^{13} \mathbf{C}$ NMR $(100$ $\left.\mathrm{MHz}, \mathrm{CDCl}_{3}\right) \delta{ }^{13} \mathrm{C} \mathrm{NMR}\left(100 \mathrm{MHz}, \mathrm{CDCl}_{3}\right) \delta 148.4,148.1,140.4,138.9,136.7,136.1,134.2$, 
128.7, 127.6, 127.5, 126.9, 123.6, $90.8\left(\mathrm{dd}, J_{\mathrm{C}-\mathrm{F}}=22.3,12.5 \mathrm{~Hz}\right), 35.7,35.1,32.9,26.4,26.0 .{ }^{19} \mathbf{F}$

NMR $\left(376 \mathrm{MHz}, \mathrm{CDCl}_{3}\right) \delta-90.42(\mathrm{~d}, J=42.9 \mathrm{~Hz}, 1 \mathrm{~F}),-91.05(\mathrm{~d}, J=42.8 \mathrm{~Hz}, 1 \mathrm{~F})$.

HRMS (ESI) m/z: $[\mathrm{M}+\mathrm{H}]^{+}$Calcd for $\mathrm{C}_{26} \mathrm{H}_{26} \mathrm{~F}_{2} \mathrm{~N}$ 390.2028; found 390.2024.

\section{Synthetic applications of the reaction}

A)<smiles>C=C(c1ccc(-c2ccc(Br)cc2)cc1)C(F)(F)F</smiles>

$40,3.7 \mathrm{mmol}, 1.2 \mathrm{~g}$

2, $5.4 \mathrm{mmol}$ (2.0 equiv)<smiles>FC(F)=C(CC1CCCCC1)c1ccc(-c2ccc(Br)cc2)cc1</smiles>

$34,71 \%(1.0 \mathrm{~g})$

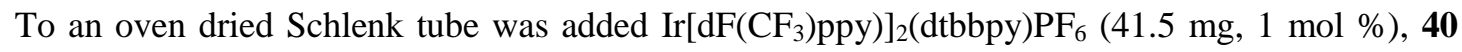
(1.2 g, 3.7 mmol, 1.0 equiv), 2 ( $0.70 \mathrm{~mL}, 2.0$ equiv), $\mathrm{K}_{3} \mathrm{PO}_{4}(1.13 \mathrm{~g}, 2.0$ equiv), triethylamine (3, $0.75 \mathrm{Ml}, 2.0$ equiv) and DCE $(37 \mathrm{~mL}, 0.1 \mathrm{M})$. The mixture was then stirred rapidly and irradiated with two $36 \mathrm{~W}$ Blue LEDs (approximately $2 \mathrm{~cm}$ away from the light source) at room temperature for $24 \mathrm{~h}$. The reaction mixture was diluted with $60 \mathrm{~mL}$ of aqueous $1 \mathrm{M} \mathrm{NaHCO}_{3}$ solution, and extracted with DCM $(3 \times 100 \mathrm{~mL})$. The combined organic extracts were washed with brine (200 $\mathrm{mL}$ ), dried over $\mathrm{Na}_{2} \mathrm{SO}_{4}$, and concentrated in vacuo. After purification by flash column chromatography on silica gel, the product was obtained in $71 \%$ yield $(1.0 \mathrm{~g})$.

B)<smiles>FC(F)=C(CCCl)c1ccc(-c2ccc(Br)cc2)cc1</smiles>

34, 1.0 equiv

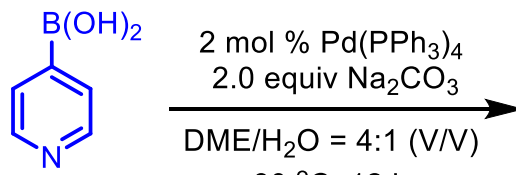

$80^{\circ} \mathrm{C}, 12 \mathrm{~h}$

1.1 equiv<smiles>FC(F)=C(CCl)c1ccc(-c2ccc(-c3cccnc3)cc2)cc1</smiles>

$41,75 \%$

According to literature reports, ${ }^{4}$ to a Schlenk tube equipped with stir bar, $\mathbf{3 3}$ (1 equiv., $0.3 \mathrm{mmol}$ ), pyridin-3-ylboronic acid (1.1 equiv., $0.39 \mathrm{mmol}), \mathrm{Na}_{2} \mathrm{CO}_{3}$ (2 equiv., $\left.0.6 \mathrm{mmol}\right)$ and $\mathrm{Pd}\left(\mathrm{PPh}_{3}\right)_{4}(2$ $\mathrm{mol} \%, 0.006 \mathrm{mmol}$ ) were added. The vessel was evacuated and filled with argon (three times), and then $\mathrm{DME} / \mathrm{H}_{2} \mathrm{O}=4: 1(5 \mathrm{~mL}, \mathrm{~V} / \mathrm{V})$ were added. Then the solution was stirred at $80{ }^{\circ} \mathrm{C}$ with heating mantle for 12 hours (TLC tracking detection). Brine $(10 \mathrm{~mL})$ was added and the aqueous layer 
was extracted with EtOAc $(3 \times 10 \mathrm{~mL})$. The combined organic layers were dried $\left(\mathrm{Na}_{2} \mathrm{SO}_{4}\right)$ and the solvent was removed under reduced pressure. The residue was purified by column chromatography $(\mathrm{PE} / \mathrm{EA}=1: 1)$ to afford yellow oil $41(58.4 \mathrm{mg}, 75 \%)$.

\section{References}

[1] Lowry M, Goldsmith J, Slinker J, Rohl R, Pascal R, Malliaras G, Bernhard S. Chem. Mater. 2005, 17: 5712.

[2] Rezazadeh S, Devannah V, Watson D. J. Am. Chem. Soc. 2017, 139: 8110; Wang X, Zhu B, Dong J, Tian H, Liu Y, Song H and Wang Q. Chem. Commun., 2021,57, 5028; Nugent J, Arroniz C, Shire B. R., Sterling A. J., Pickford H. D., Wong M. L. J., Mansfield S. J., Caputo D. F. J., Owen B., Mousseau J. J., Duarte F. and Anderson E. A. ACS Catal. 2019, 9, 9568.

[3] Xia P, Ye Z, Hu Y, Song D, Xiang H, Chen, Yang H. Org. Lett. 2019, 21: 2658.

[4] Voets M, Antes I, Scherer C, Muller-Vieira U, Biemel K, Barassin C, Marchais-Oberwinkler S, Hartmann R. J. Med. Chem. 2005, 48: 6632-6642.

\section{NMR Spectra}

${ }^{1} \mathrm{H}$ NMR $\left(400 \mathrm{MHz}, \mathrm{CDCl}_{3}\right)$ spectrum of compound 4
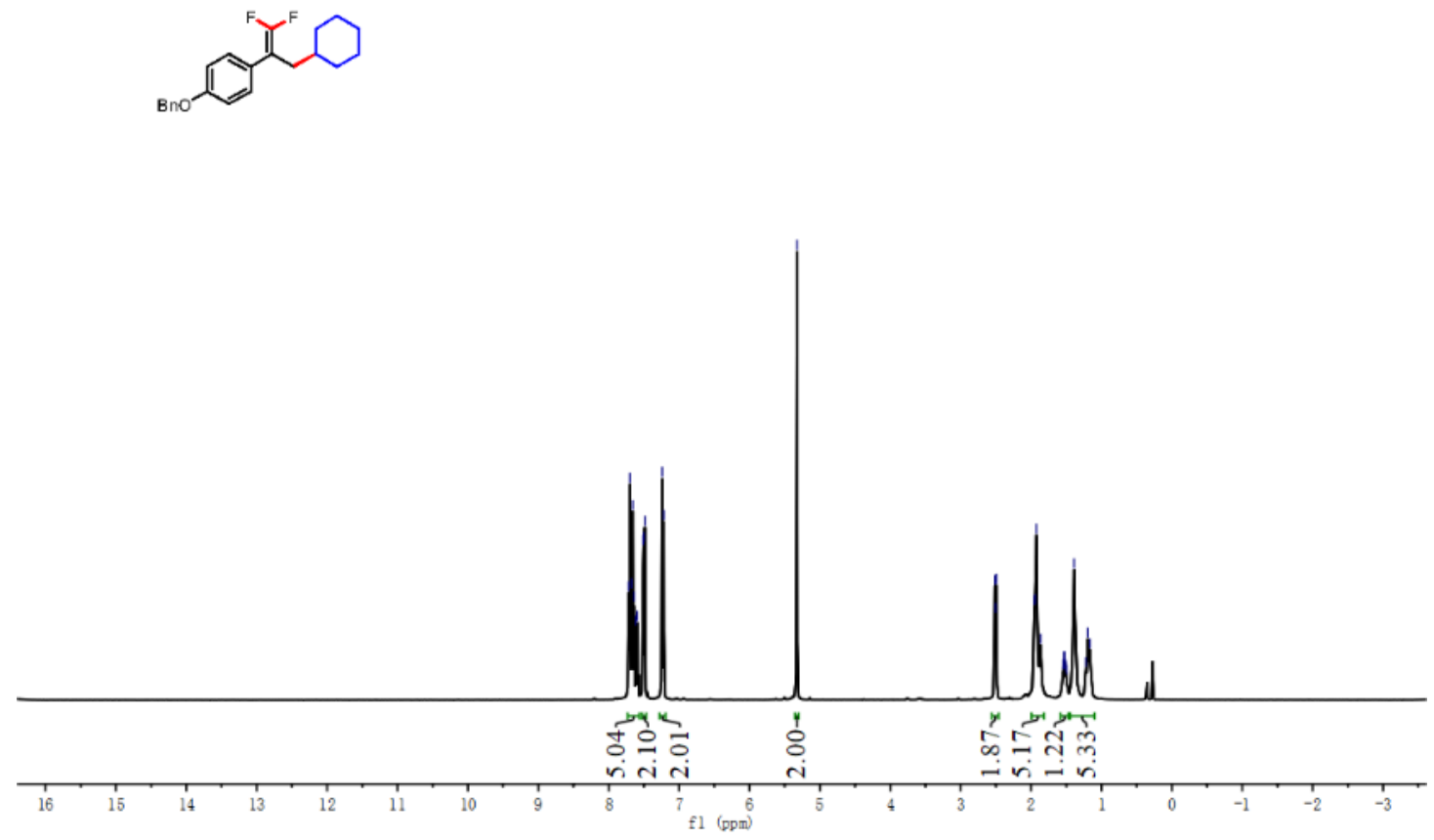

${ }^{13} \mathrm{C}$ NMR (100 MHz, $\left.\mathrm{CDCl}_{3}\right)$ spectrum of compound 4 
YFY-3-119
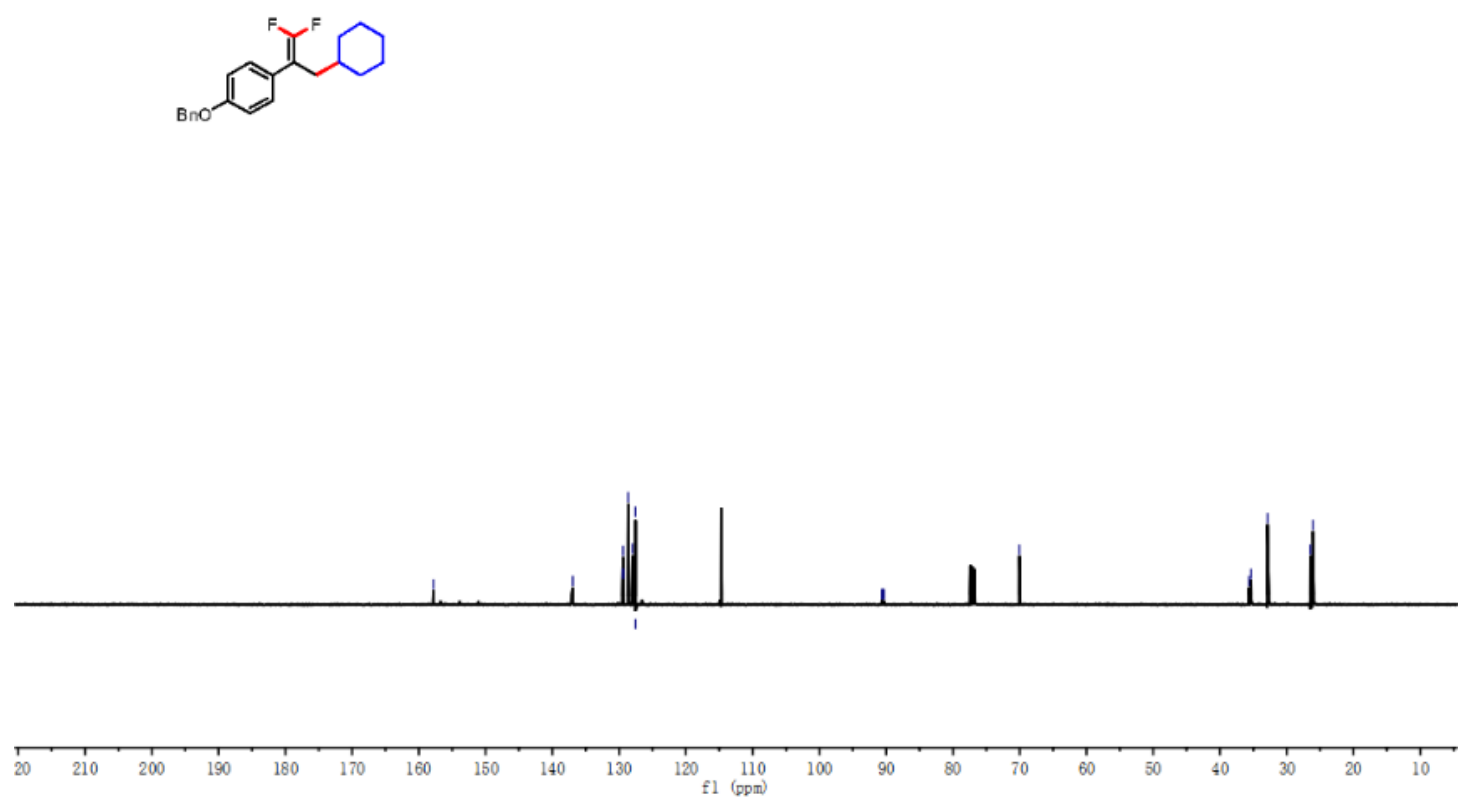

${ }^{19} \mathrm{~F}$ NMR (376 MHz, $\mathrm{CDCl}_{3}$ ) spectrum of compound 4
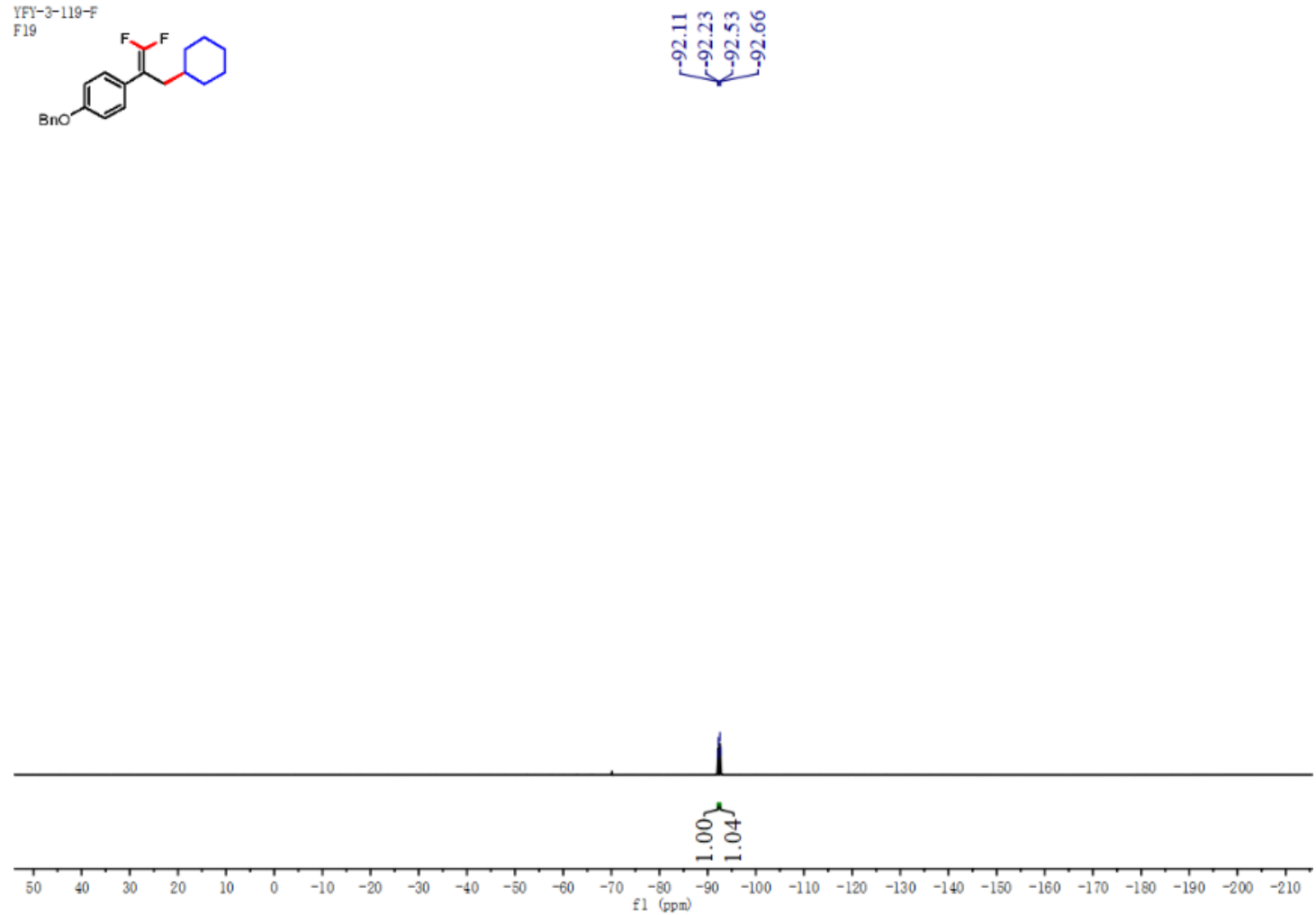

${ }^{1} \mathrm{H}$ NMR (400 MHz, $\mathrm{CDCl}_{3}$ ) spectrum of compound $\mathbf{5}$ 

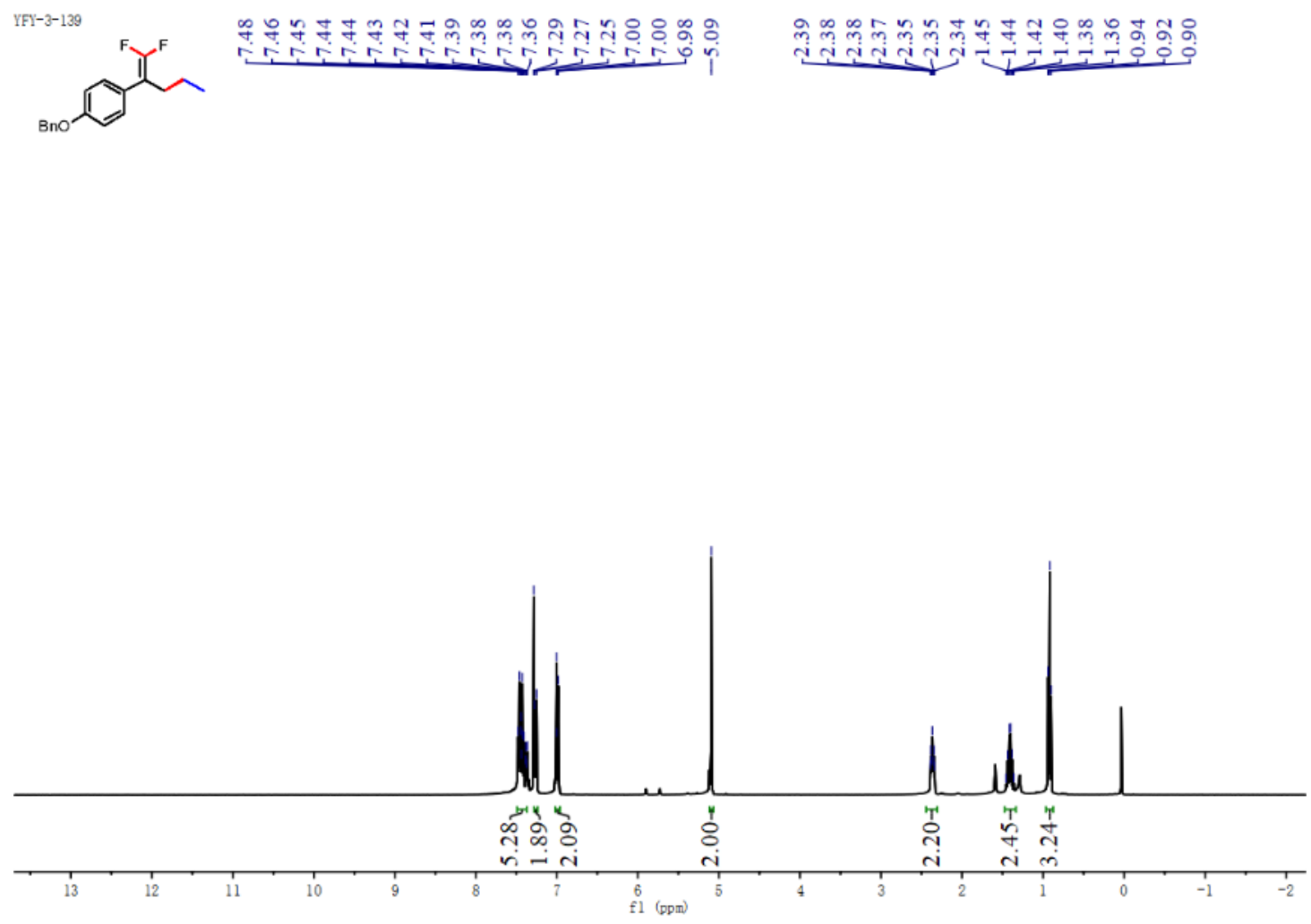

${ }^{13} \mathrm{C} \mathrm{NMR}\left(100 \mathrm{MHz}, \mathrm{CDCl}_{3}\right)$ spectrum of compound 5

Bno

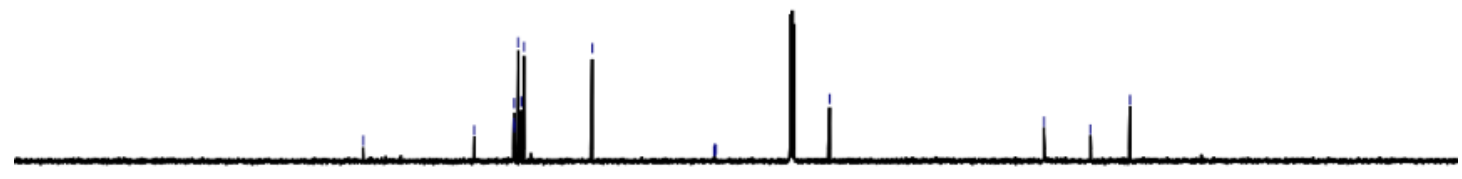

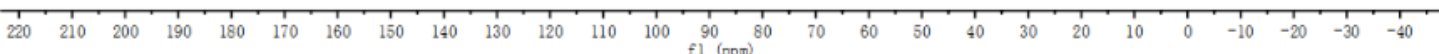

${ }^{19} \mathrm{~F}$ NMR (376 MHz, $\mathrm{CDCl}_{3}$ ) spectrum of compound 5 


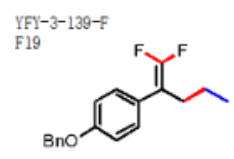

-

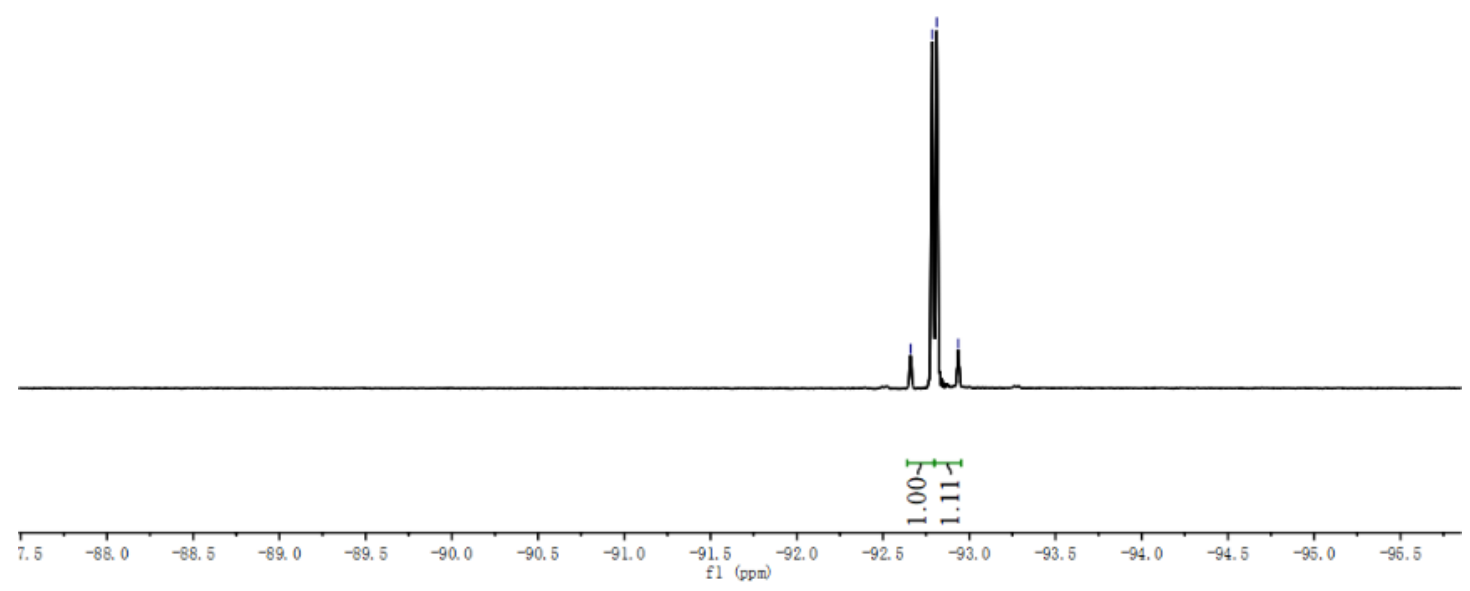

${ }^{1} \mathrm{H}$ NMR (400 MHz, $\mathrm{CDCl}_{3}$ ) spectrum of compound 6
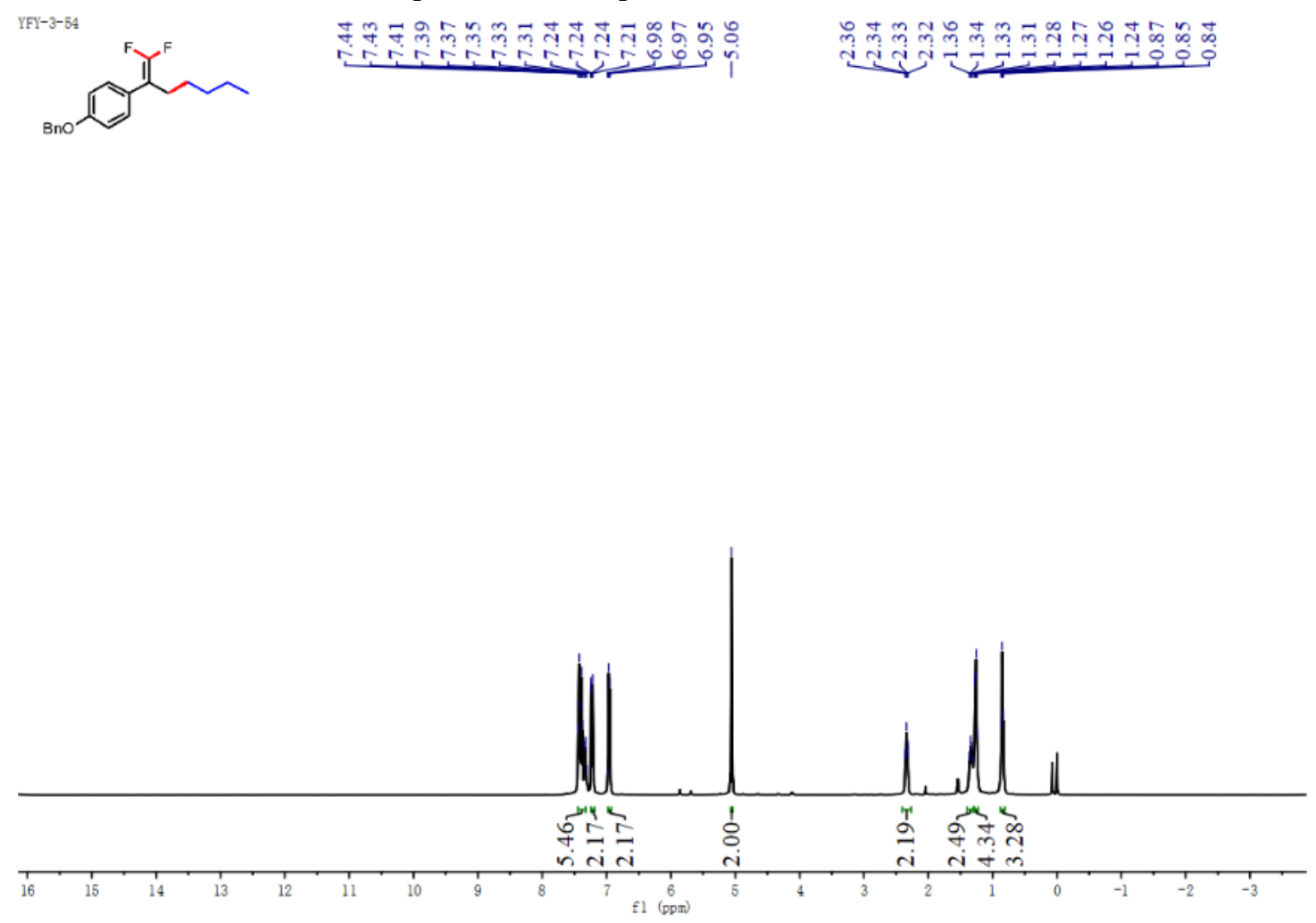

${ }^{13} \mathrm{C}$ NMR (100 MHz, $\mathrm{CDCl}_{3}$ ) spectrum of compound 6 

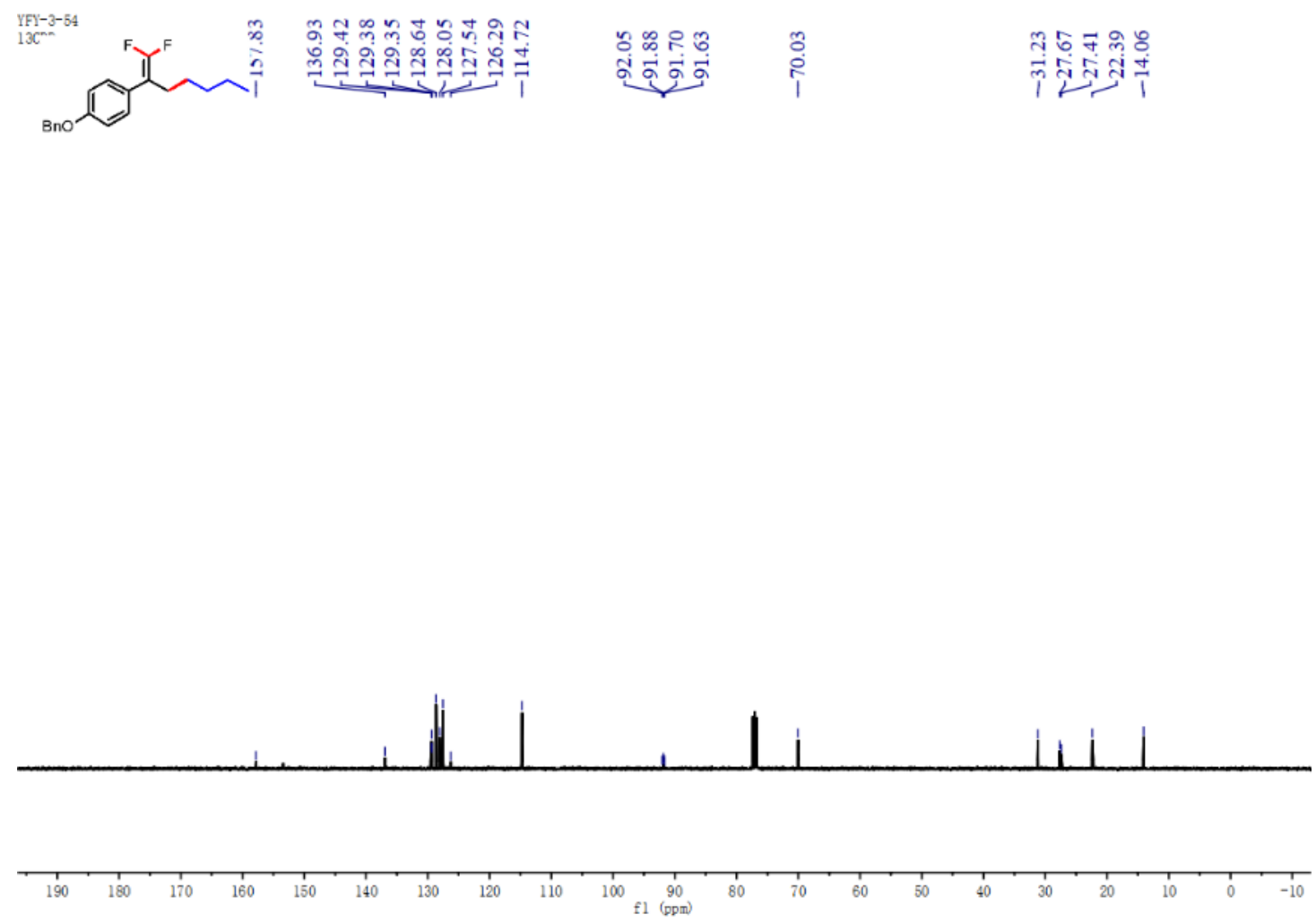

${ }^{19} \mathrm{~F}$ NMR (376 MHz, $\left.\mathrm{CDCl}_{3}\right)$ spectrum of compound 6
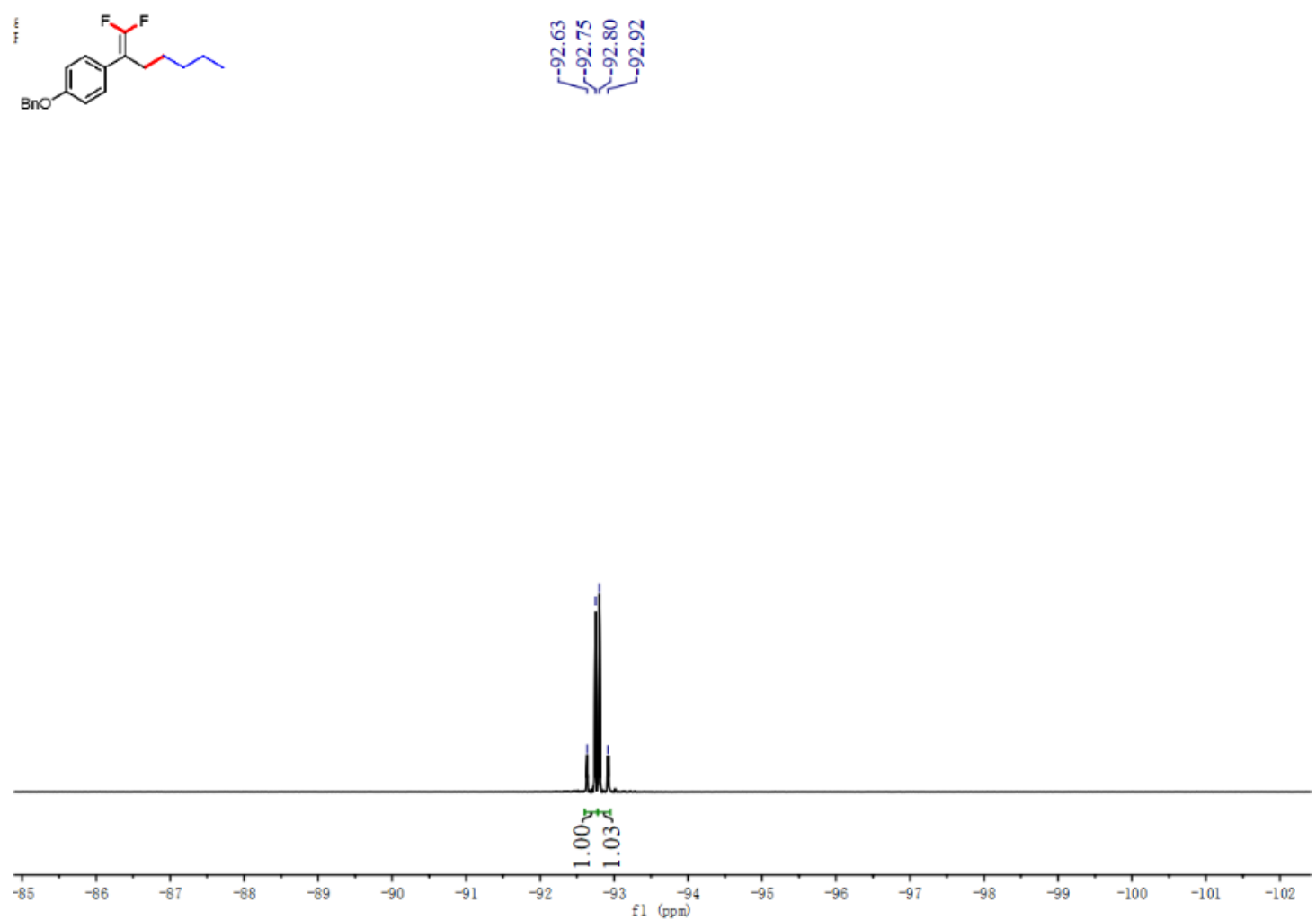

${ }^{1} \mathrm{H}$ NMR (400 MHz, $\mathrm{CDCl}_{3}$ ) spectrum of compound 7 
<smiles>CC(C)CC(=C1C=CC=C1)C(C)(C)C</smiles>

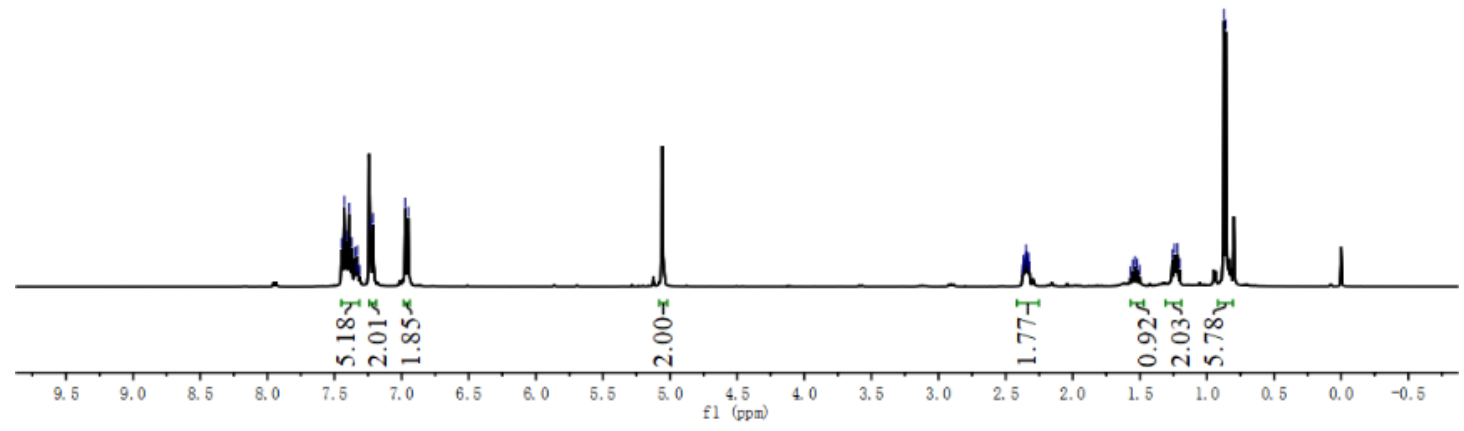

${ }^{13} \mathrm{C}$ NMR (100 MHz, $\left.\mathrm{CDCl}_{3}\right)$ spectrum of compound 7

\begin{tabular}{|c|c|c|c|c|c|}
\hline $\begin{array}{l}\text { YFFr-3-53 } \\
13 C^{-3}\end{array}$ & $\begin{array}{l}\infty \\
\stackrel{\infty}{5} \\
i \\
1\end{array}$ & 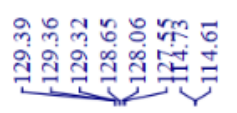 & 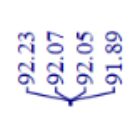 & $\stackrel{\text { ôे }}{i}$ & 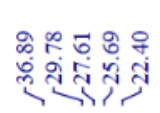 \\
\hline
\end{tabular}
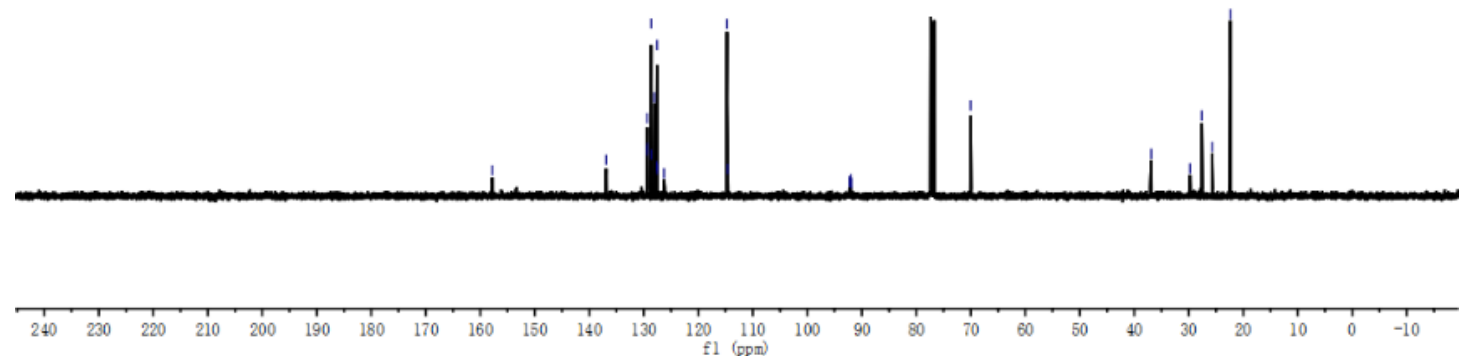

${ }^{19} \mathrm{~F} \mathrm{NMR}\left(376 \mathrm{MHz}, \mathrm{CDCl}_{3}\right.$ ) spectrum of compound 7 

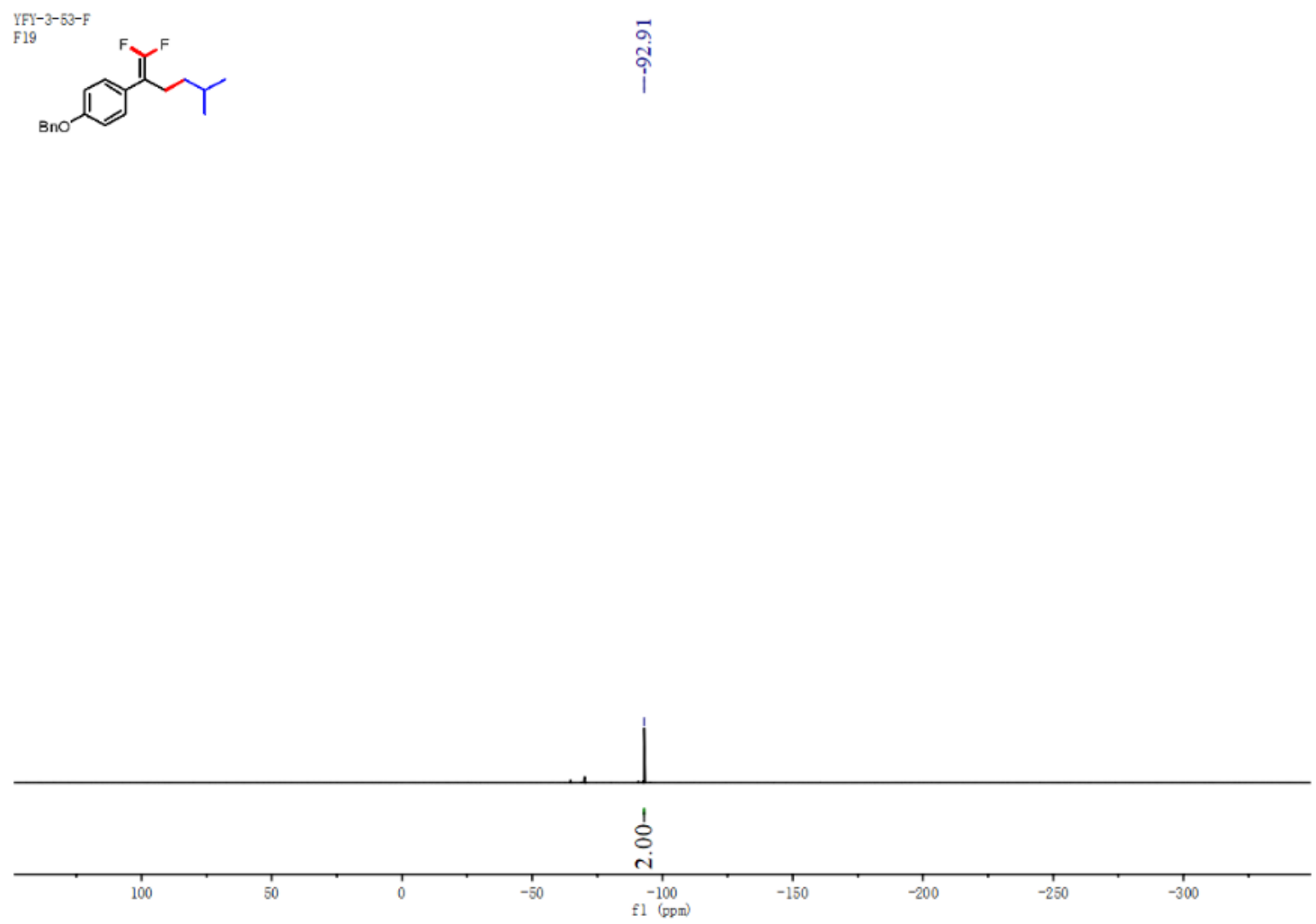

${ }^{1} \mathrm{H}$ NMR (400 MHz, $\mathrm{CDCl}_{3}$ ) spectrum of compound 8
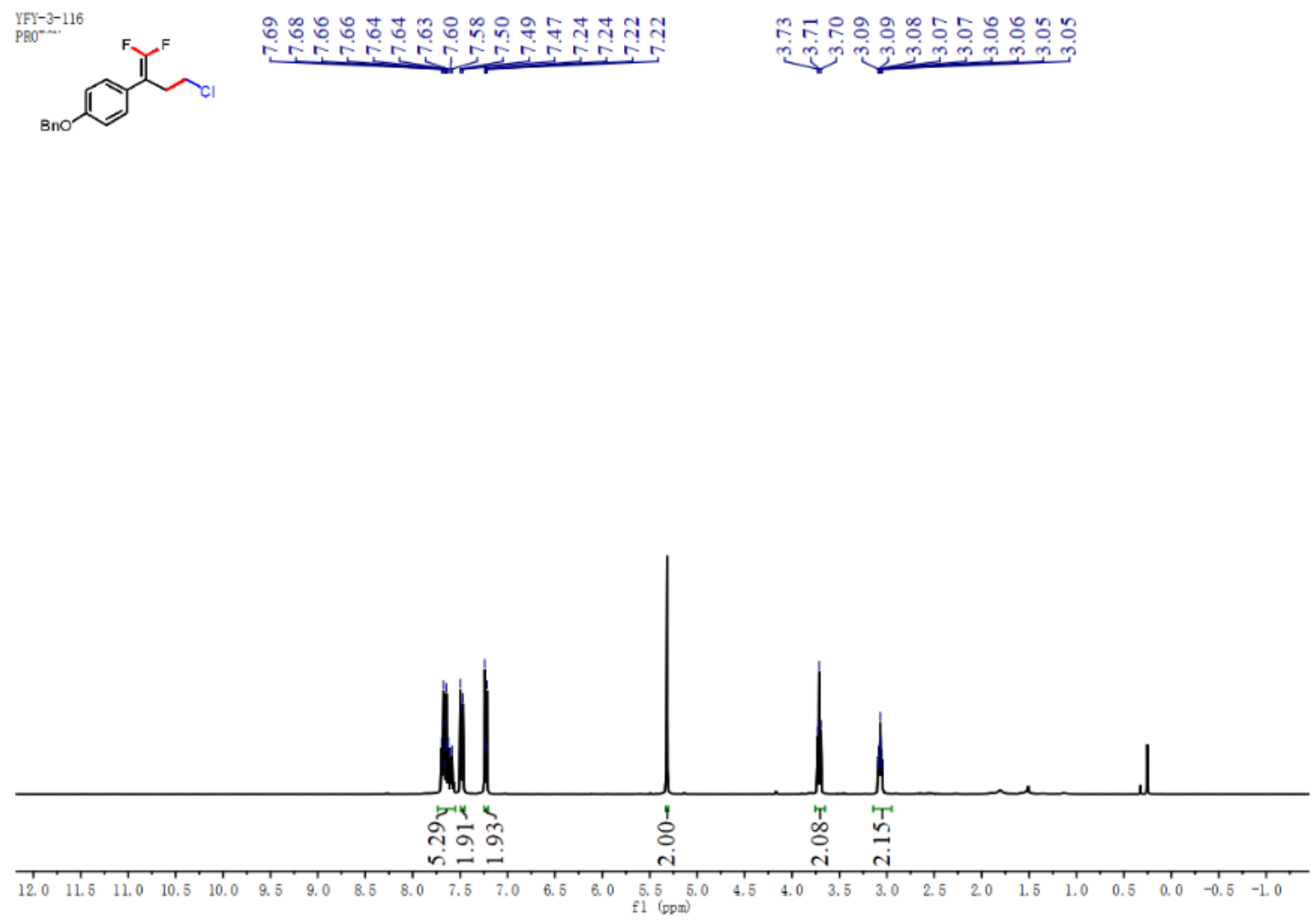

${ }^{13} \mathrm{C}$ NMR $\left(100 \mathrm{MHz}, \mathrm{CDCl}_{3}\right.$ ) spectrum of compound 8 

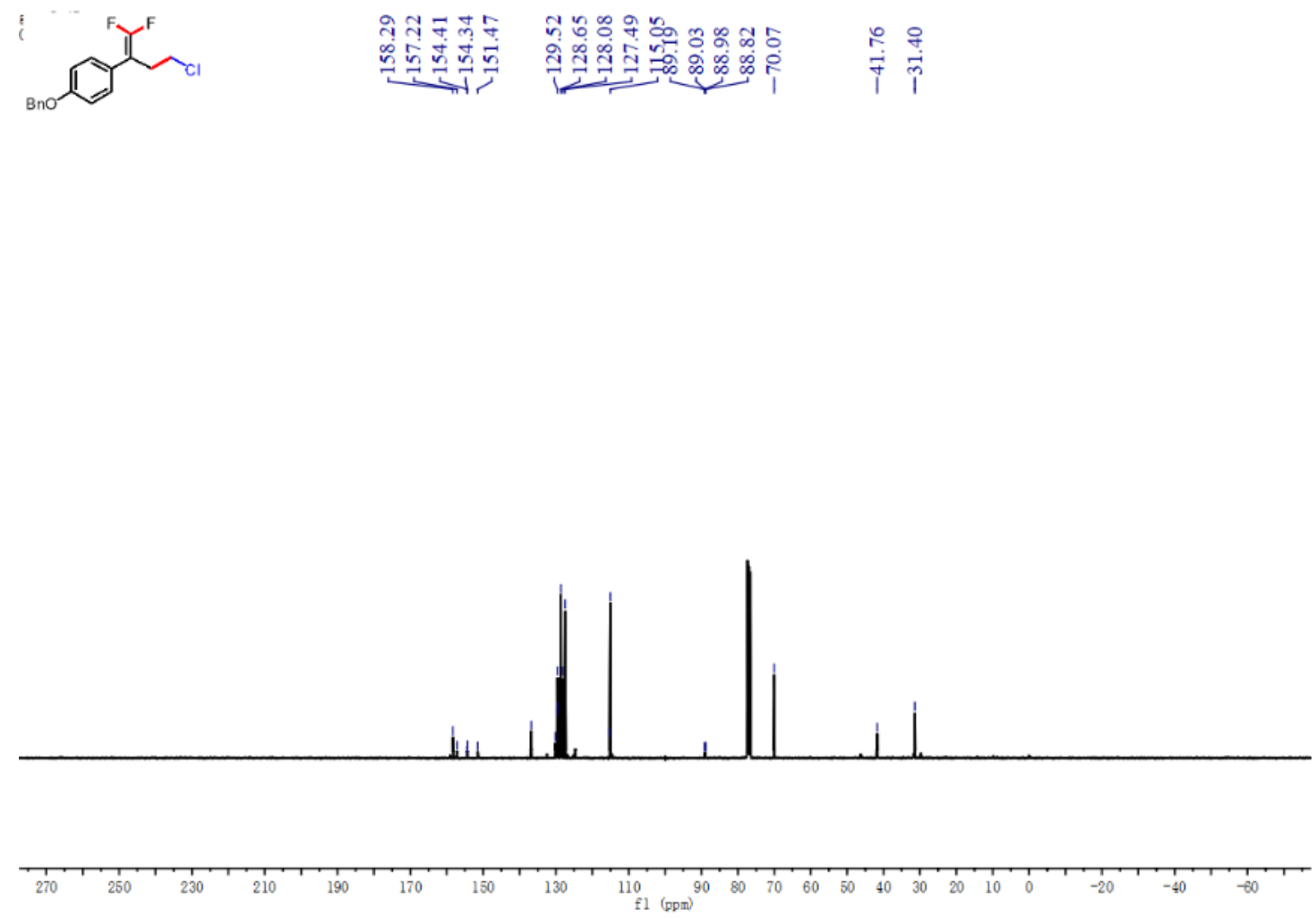

${ }^{19} \mathrm{~F}$ NMR $\left(376 \mathrm{MHz}, \mathrm{CDCl}_{3}\right.$ ) spectrum of compound 8

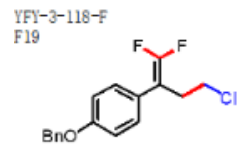

$8=\pi 0^{2}$

연요

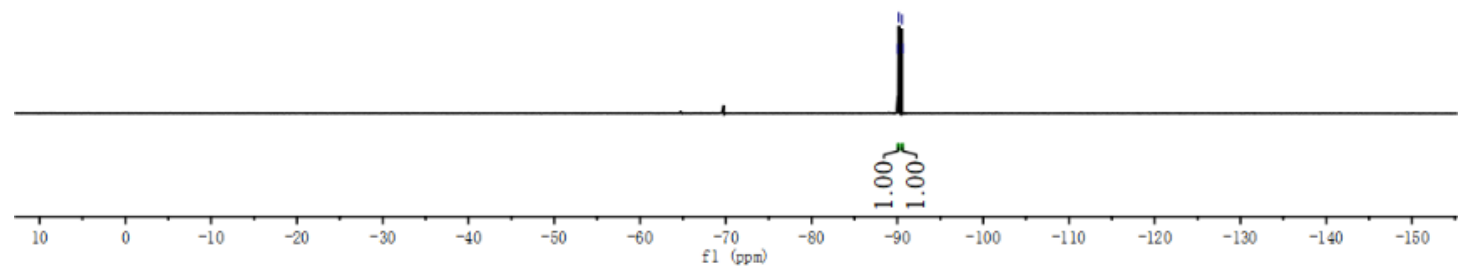

${ }^{1} \mathrm{H}$ NMR (400 MHz, $\mathrm{CDCl}_{3}$ ) spectrum of compound 9 

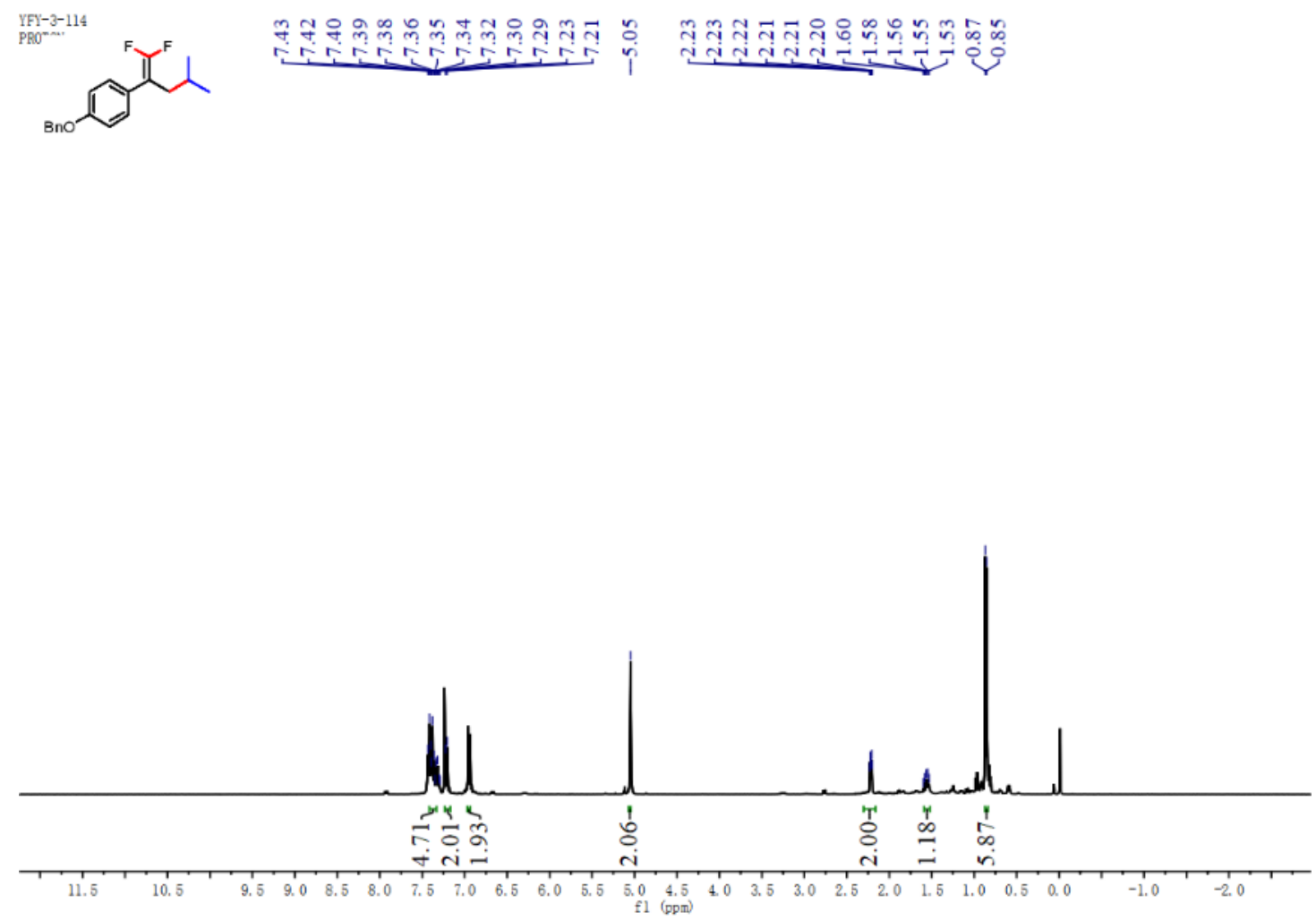

${ }^{13} \mathrm{C} \mathrm{NMR}\left(100 \mathrm{MHz}, \mathrm{CDCl}_{3}\right)$ spectrum of compound 9
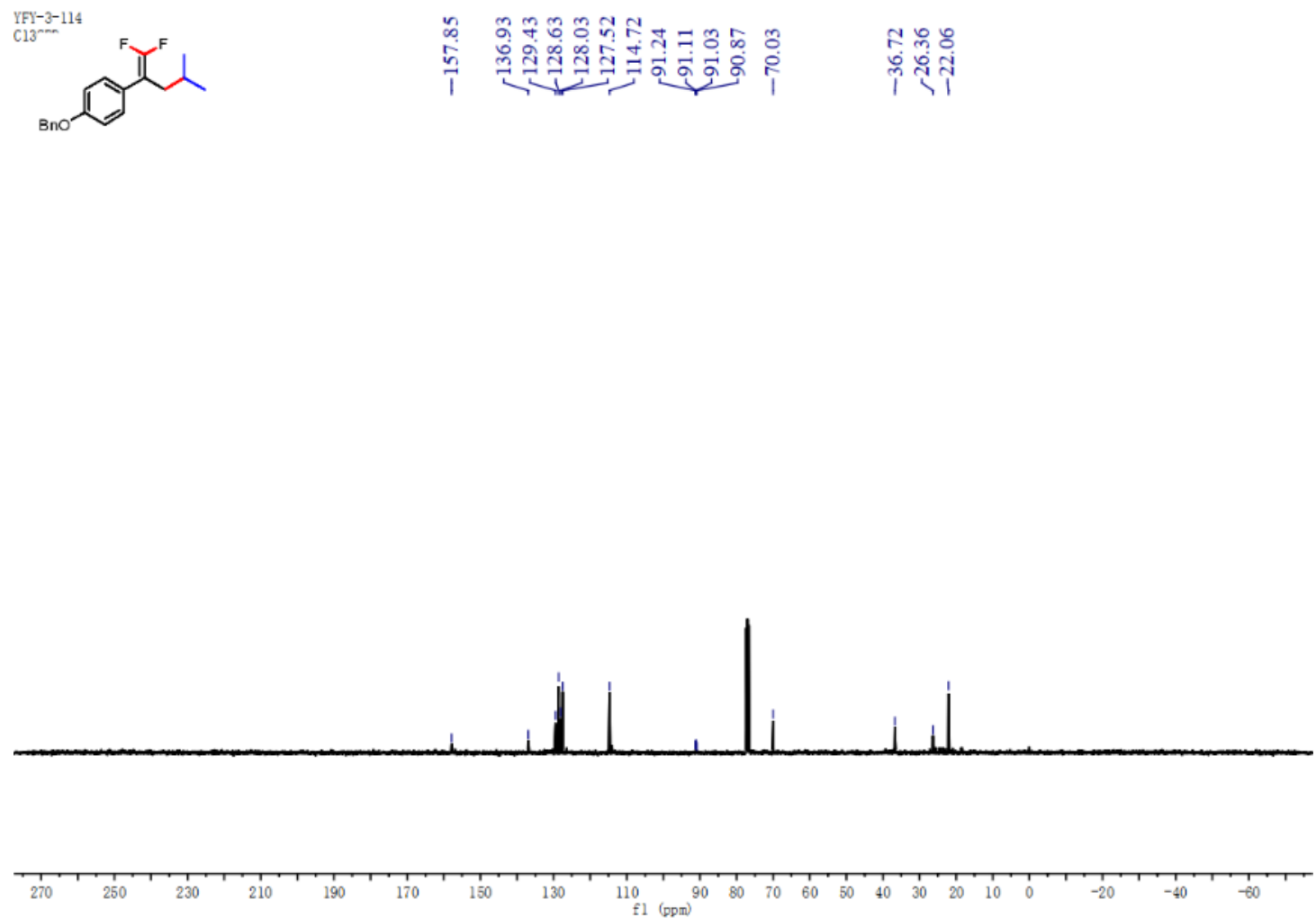

${ }^{19} \mathrm{~F}$ NMR $\left(376 \mathrm{MHz}, \mathrm{CDCl}_{3}\right.$ ) spectrum of compound 9 

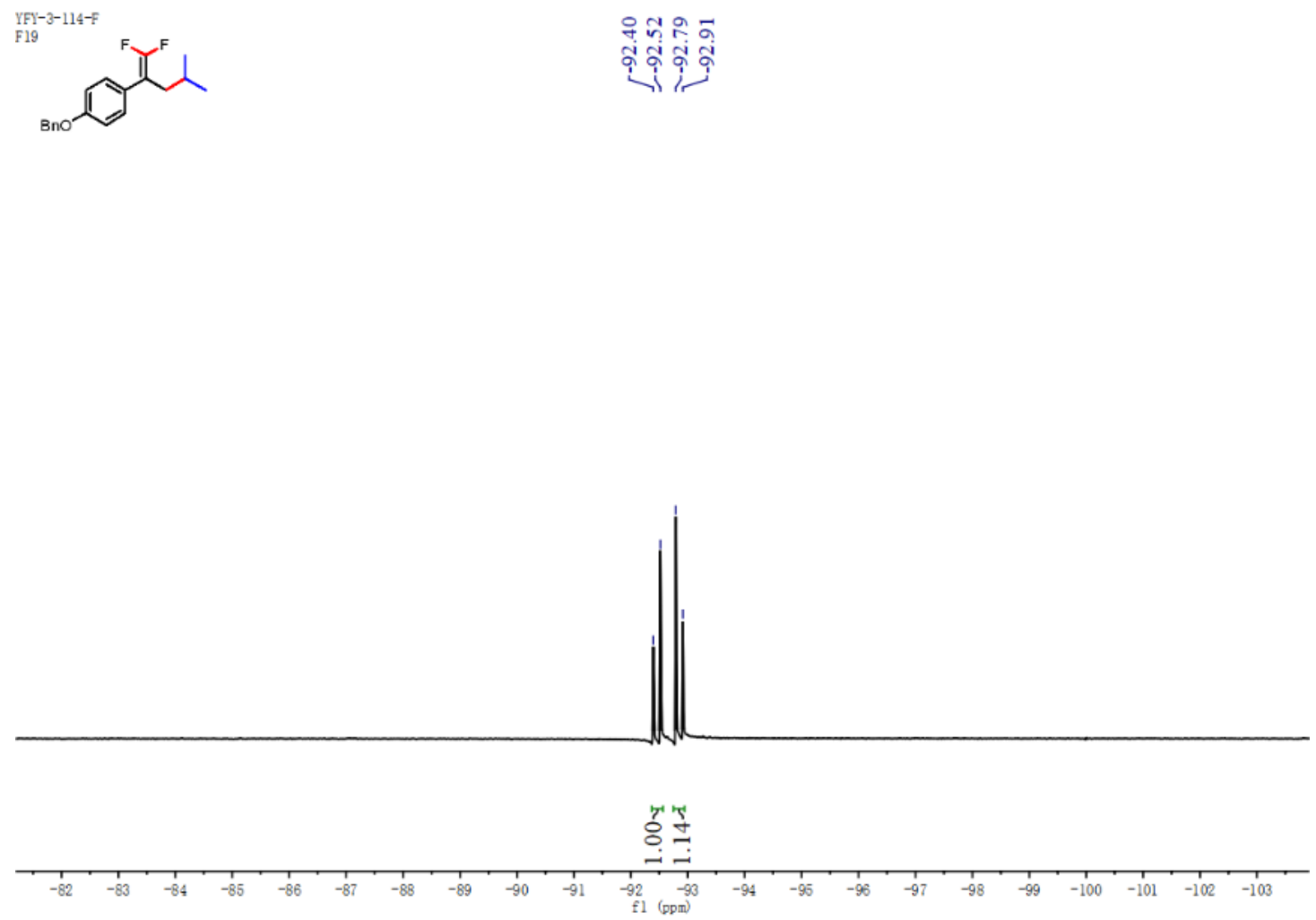

${ }^{1} \mathrm{H}$ NMR (400 MHz, $\mathrm{CDCl}_{3}$ ) spectrum of compound $\mathbf{1 0}$

YFF-3-116
Proron
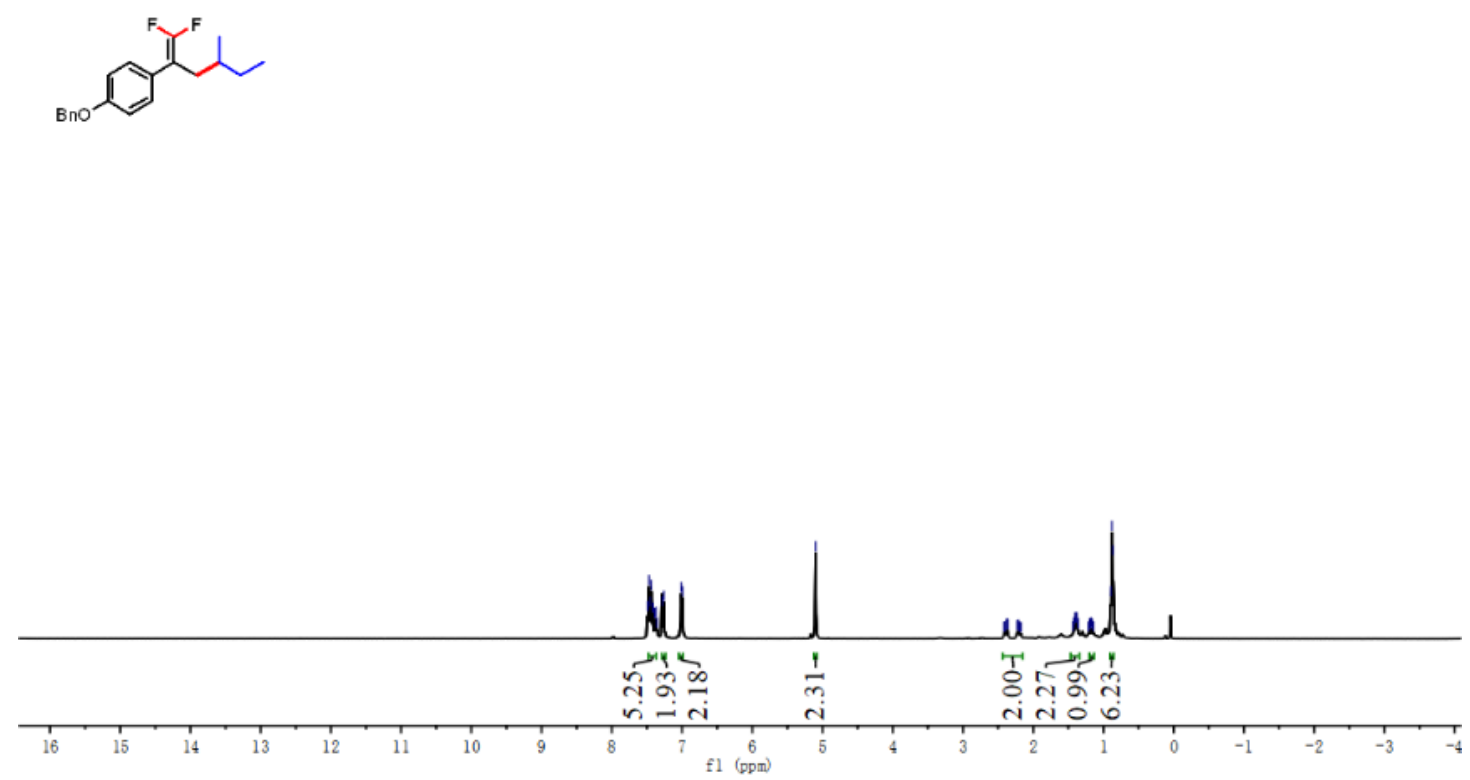

${ }^{13} \mathrm{C}$ NMR $\left(100 \mathrm{MHz}, \mathrm{CDCl}_{3}\right)$ spectrum of compound $\mathbf{1 0}$ 
年人

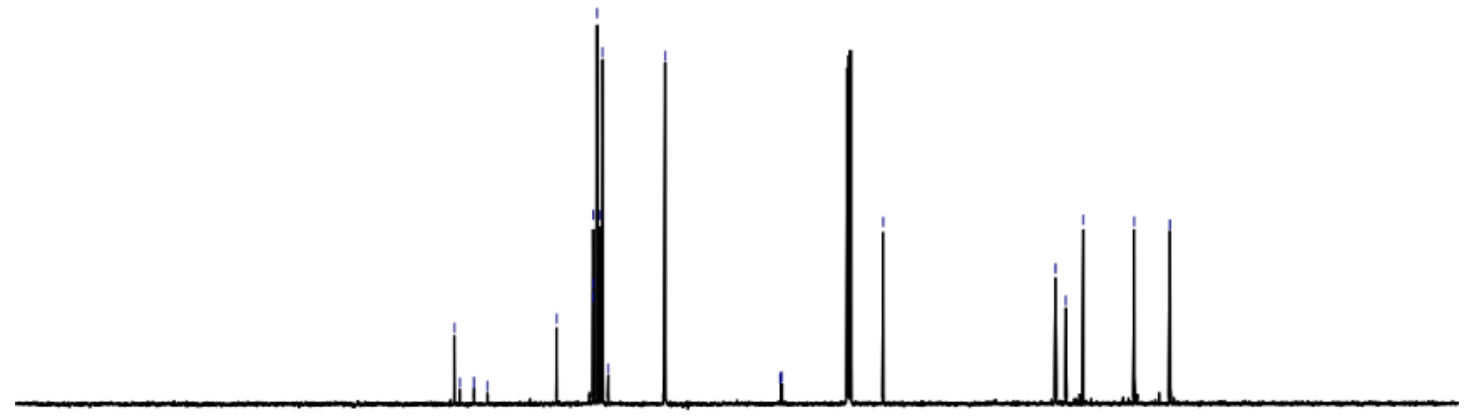

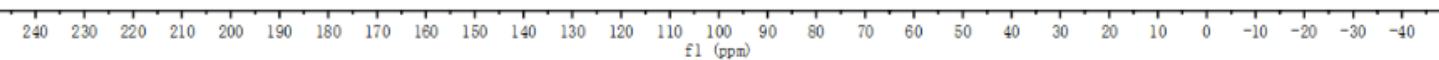

${ }^{19} \mathrm{~F}$ NMR $\left(376 \mathrm{MHz}, \mathrm{CDCl}_{3}\right)$ spectrum of compound $\mathbf{1 0}$

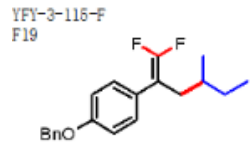

F. 药

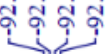

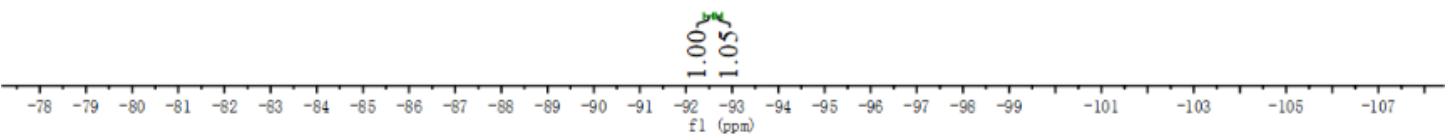

${ }^{1} \mathrm{H}$ NMR (400 MHz, $\mathrm{CDCl}_{3}$ ) spectrum of compound 11 

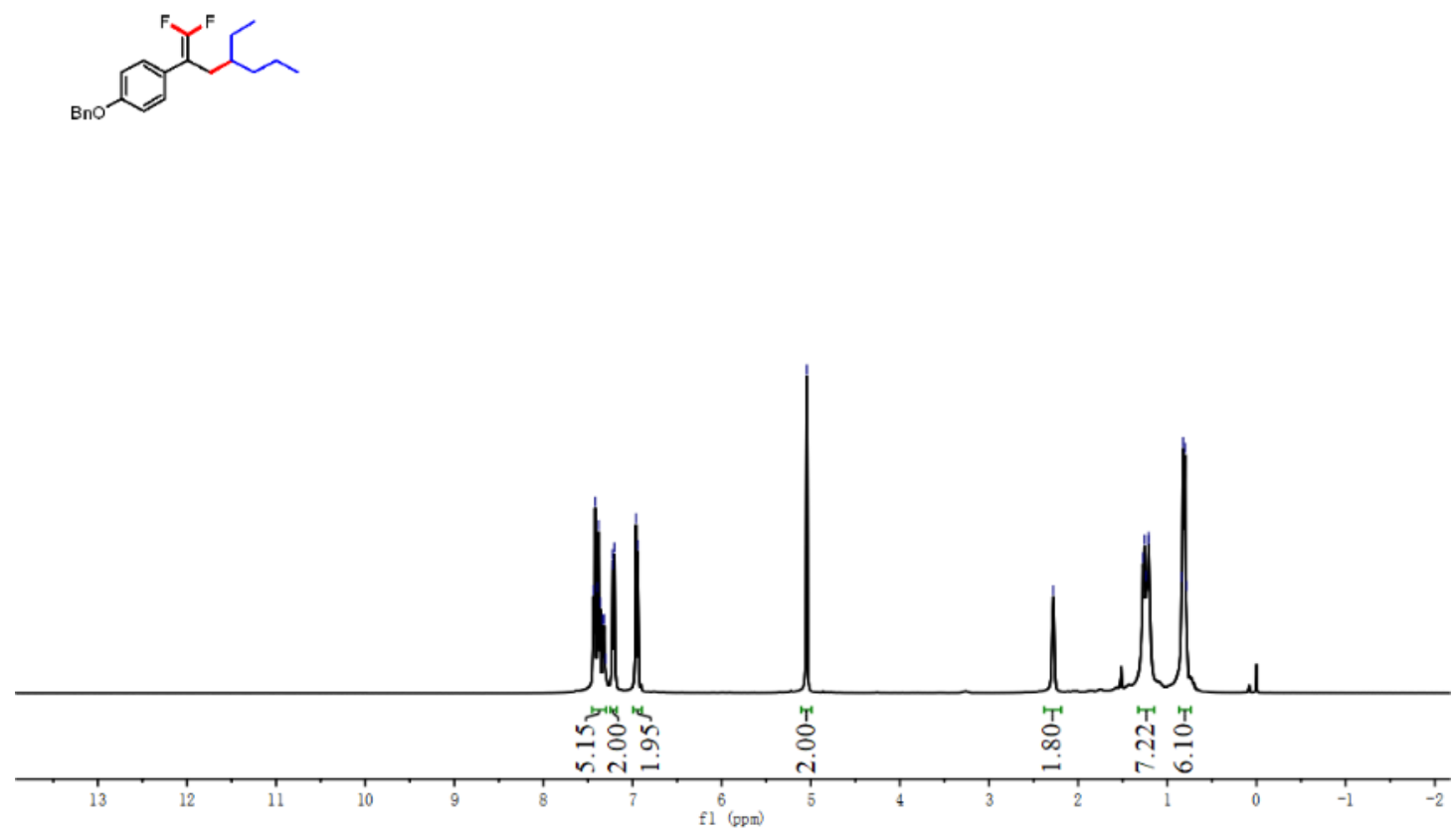

${ }^{13} \mathrm{C}$ NMR (100 MHz, $\mathrm{CDCl}_{3}$ ) spectrum of compound 11 ${ }_{13 \mathrm{CPD}}^{\mathrm{YPF}-3-45}$

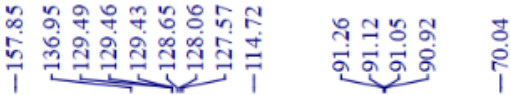

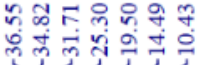<smiles>CCCCC(CCC)C(=C(C)F)c1ccc(Br)cc1</smiles>

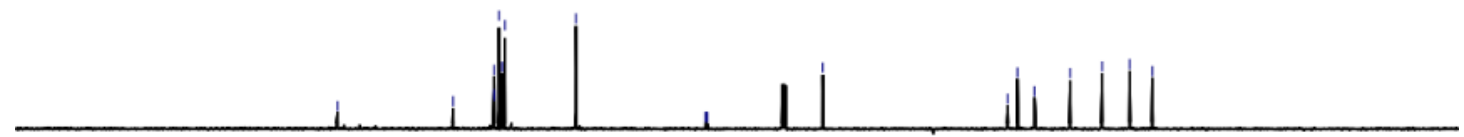

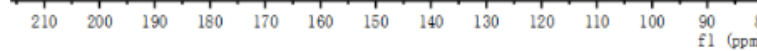

${ }^{19} \mathrm{~F}$ NMR $\left(376 \mathrm{MHz}, \mathrm{CDCl}_{3}\right)$ spectrum of compound 11 

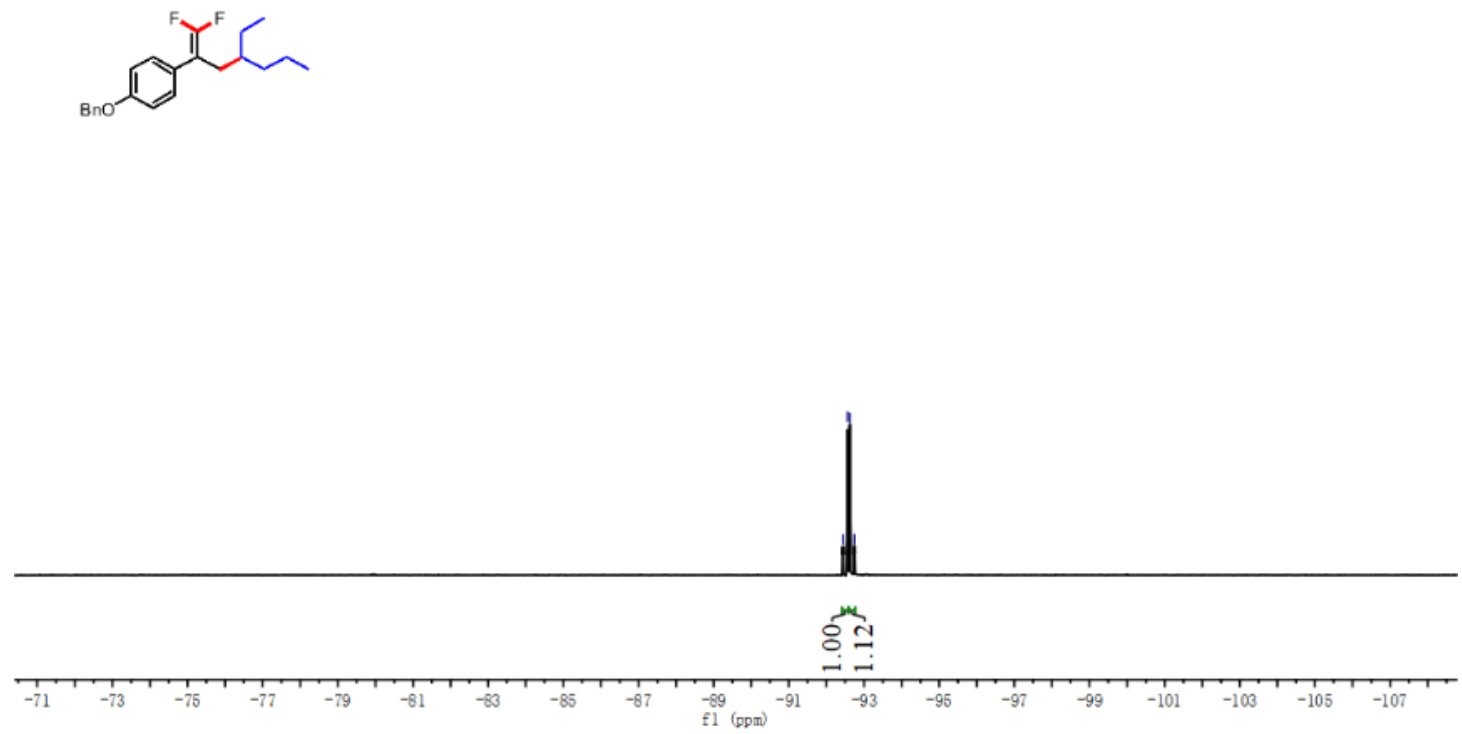

${ }^{1} \mathrm{H}$ NMR (400 MHz, $\mathrm{CDCl}_{3}$ ) spectrum of compound 12

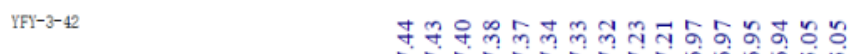

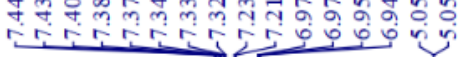
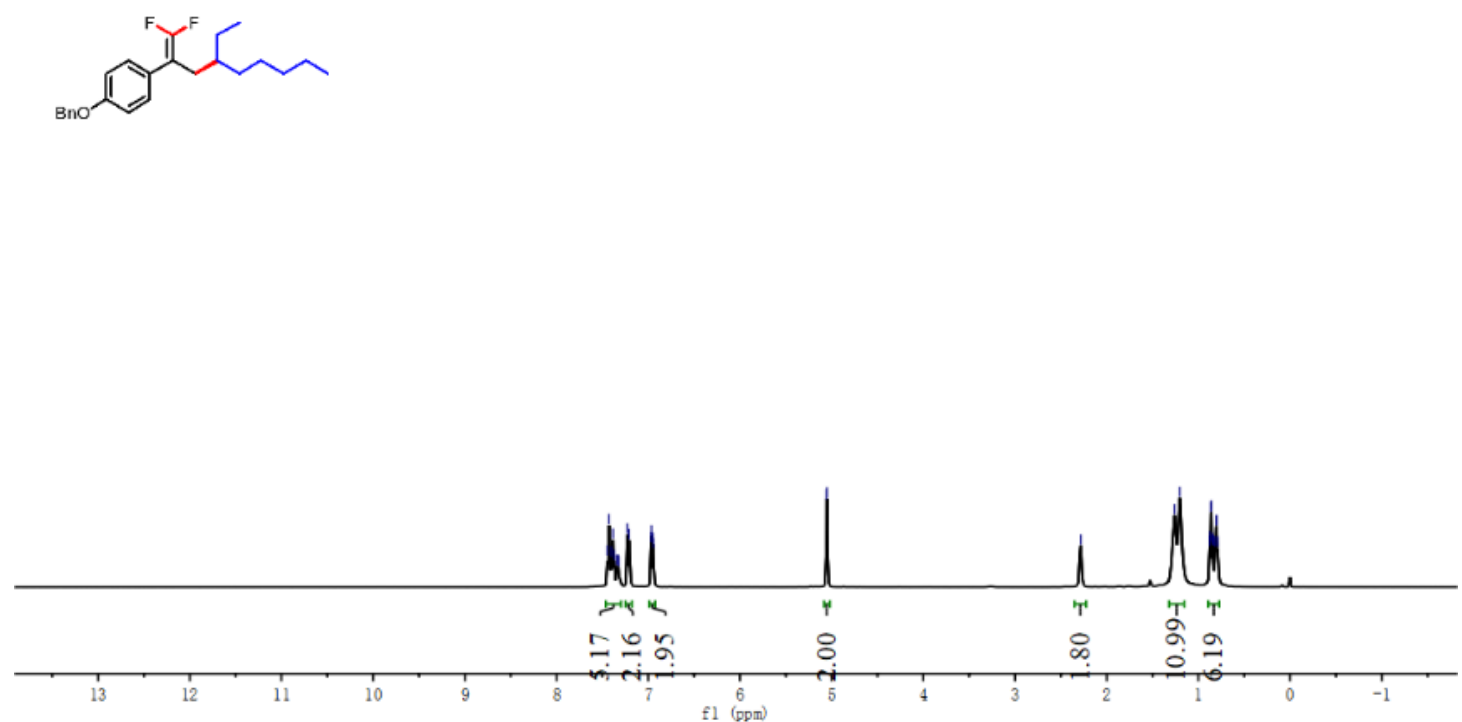

${ }^{13} \mathrm{C}$ NMR (100 MHz, $\mathrm{CDCl}_{3}$ ) spectrum of compound $\mathbf{1 2}$ 

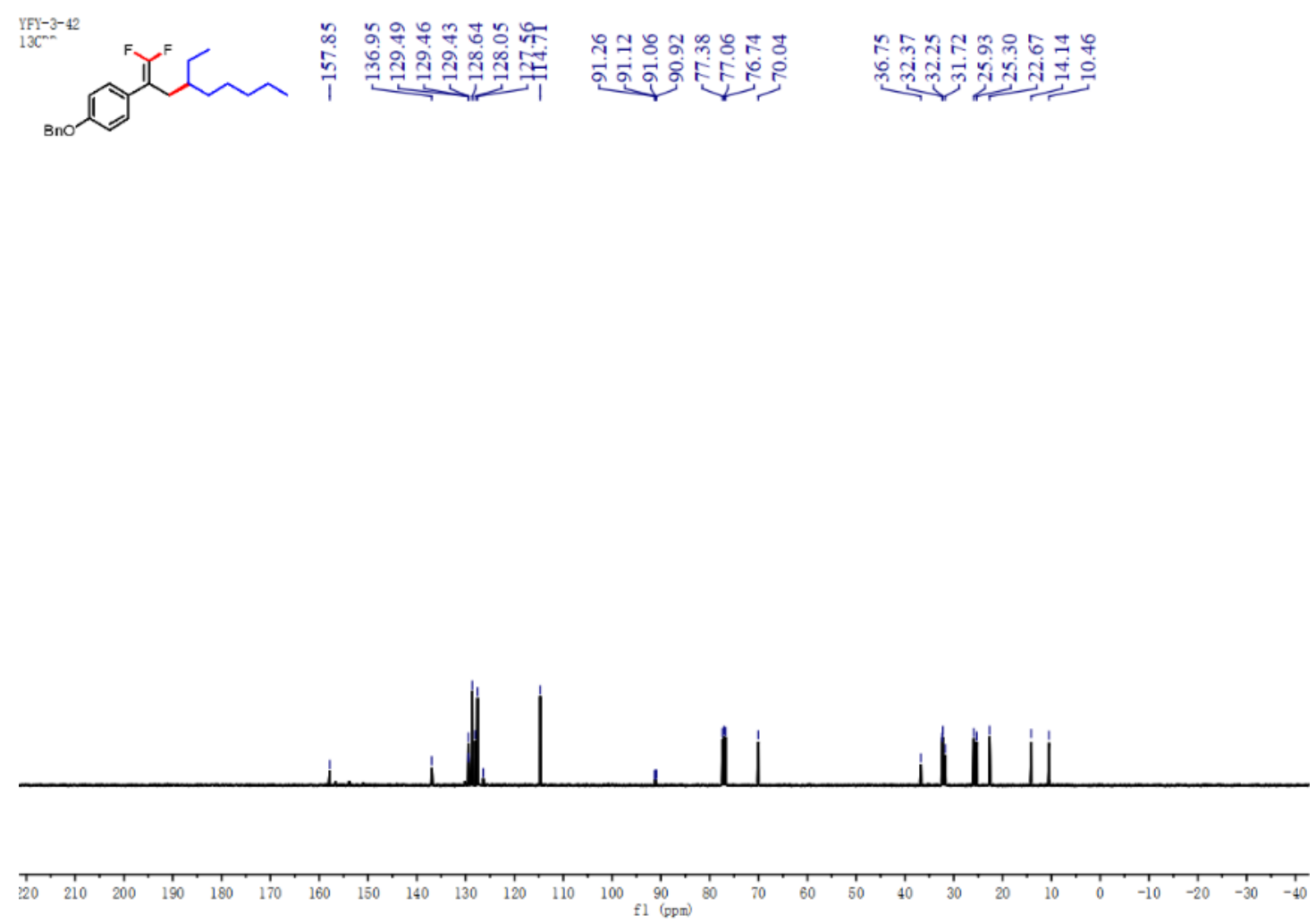

${ }^{19} \mathrm{~F} \mathrm{NMR}\left(376 \mathrm{MHz}, \mathrm{CDCl}_{3}\right.$ ) spectrum of compound 12
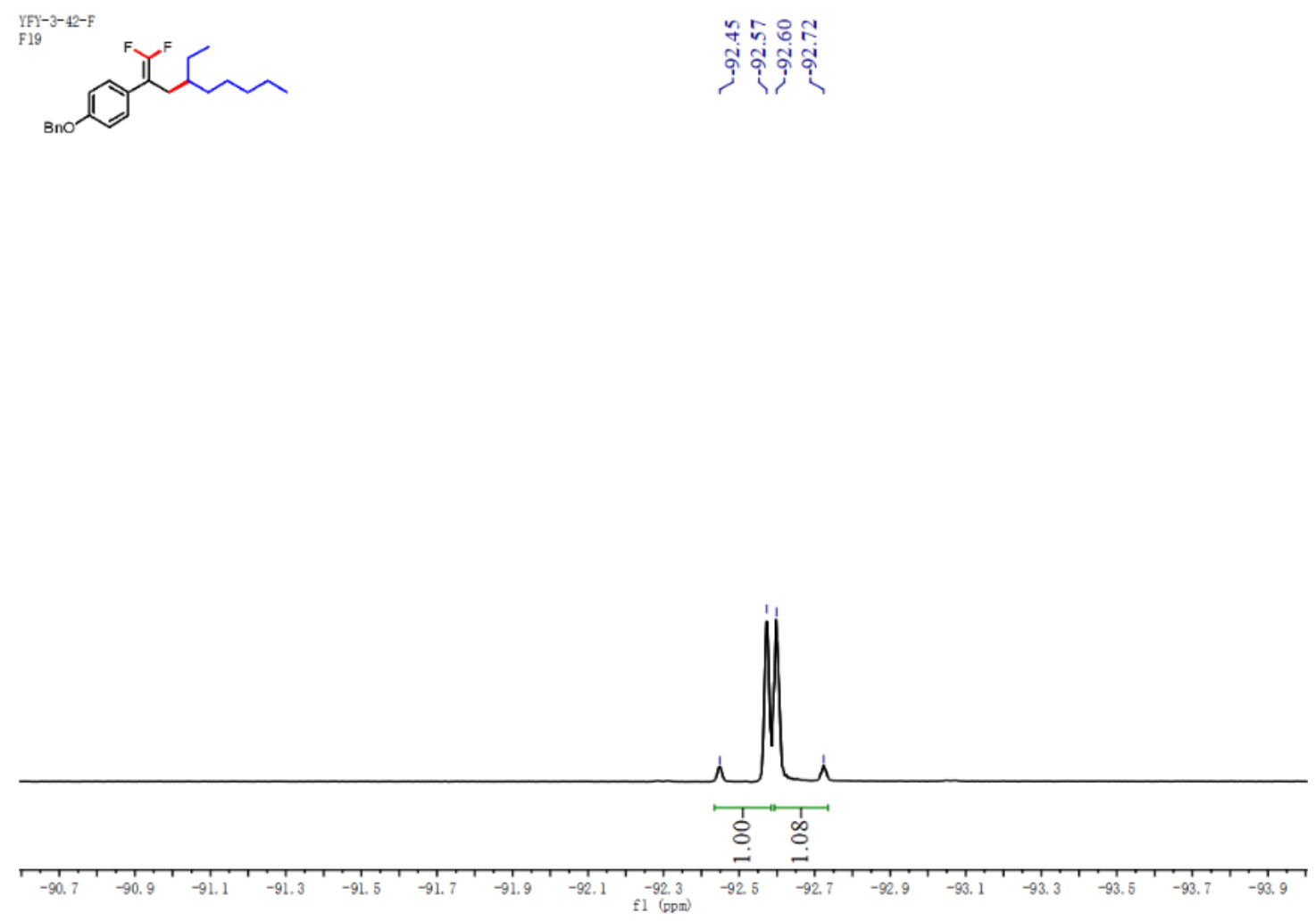

${ }^{1} \mathrm{H}$ NMR (400 MHz, $\mathrm{CDCl}_{3}$ ) spectrum of compound $\mathbf{1 3}$ 

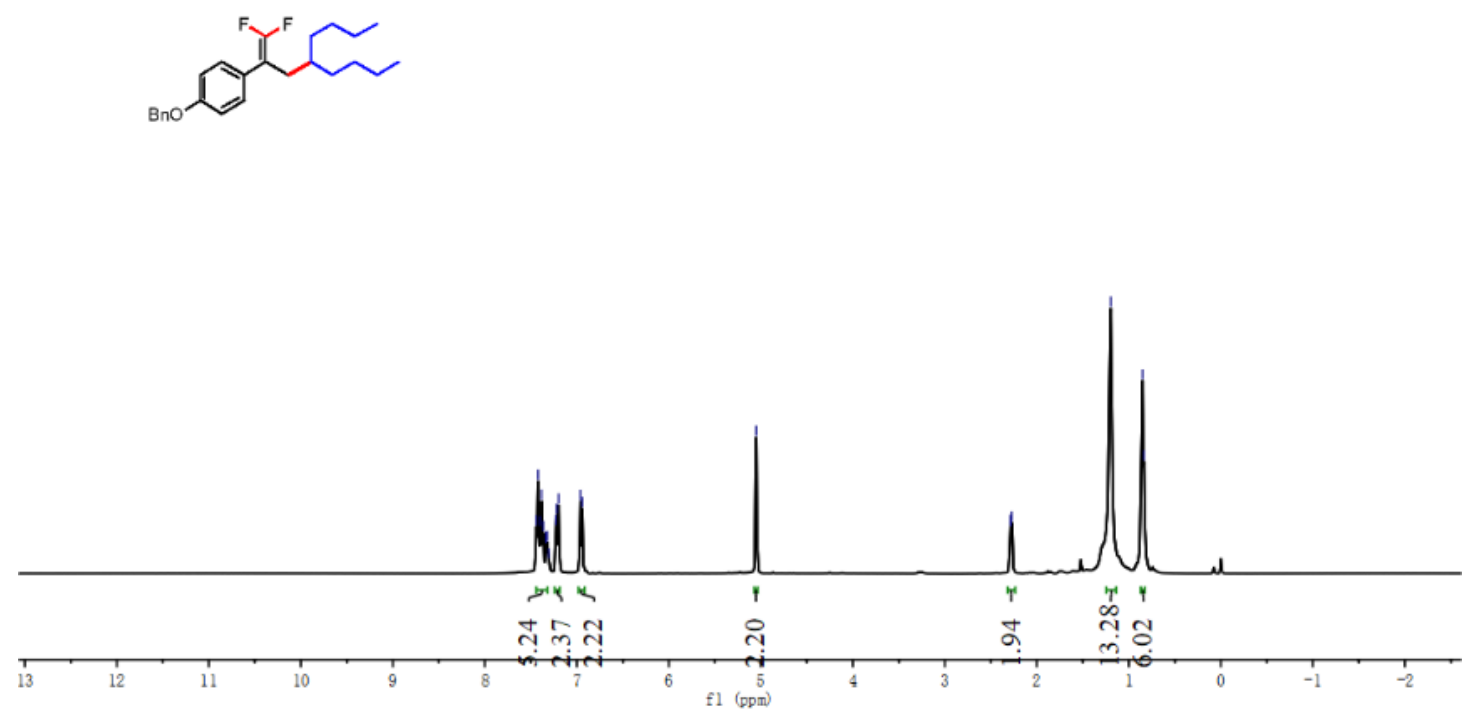

${ }^{13} \mathrm{C}$ NMR (100 MHz, $\mathrm{CDCl}_{3}$ ) spectrum of compound $\mathbf{1 3}$

\section{$\mathrm{YFF}-3-36$
$13 \mathrm{CPO}$}

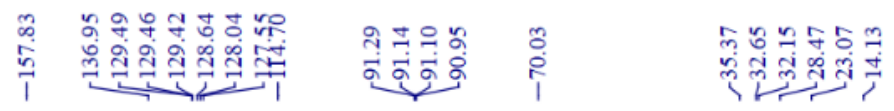
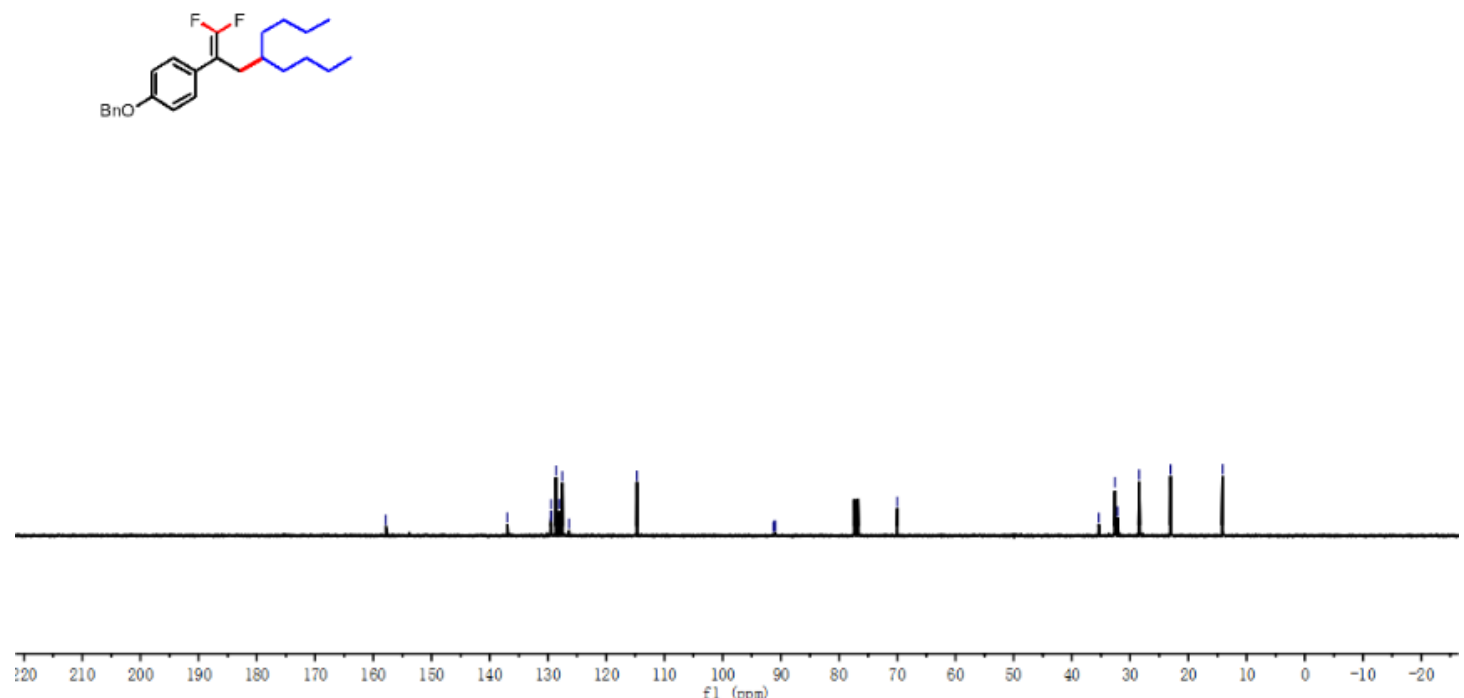

${ }^{19} \mathrm{~F}$ NMR (376 MHz, $\mathrm{CDCl}_{3}$ ) spectrum of compound $\mathbf{1 3}$ 

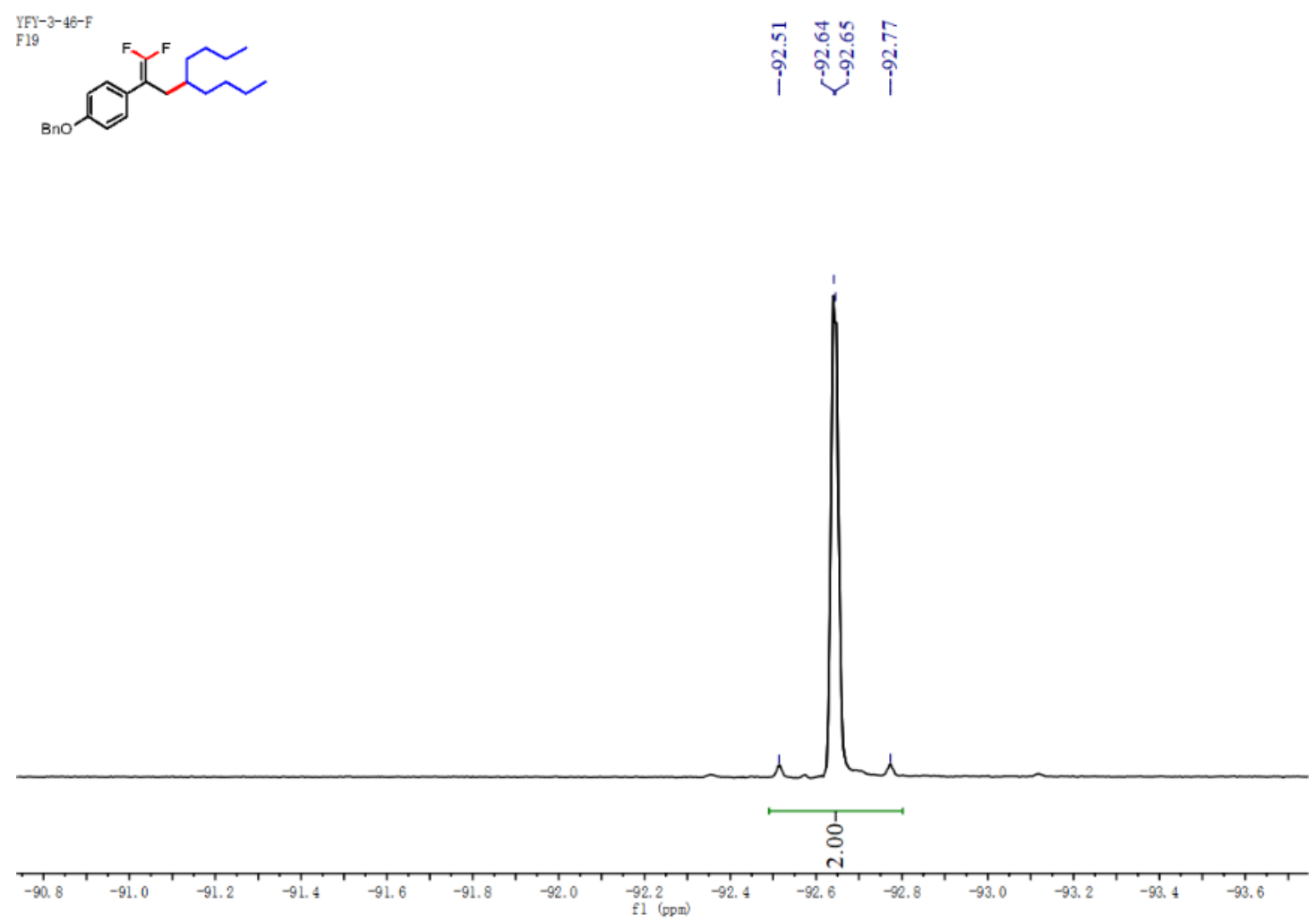

${ }^{1} \mathrm{H}$ NMR (400 MHz, $\mathrm{CDCl}_{3}$ ) spectrum of compound 14 YFY-3-57

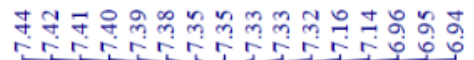<smiles>CCC(CCO)(c1ccccc1)C(C)(C)C</smiles>

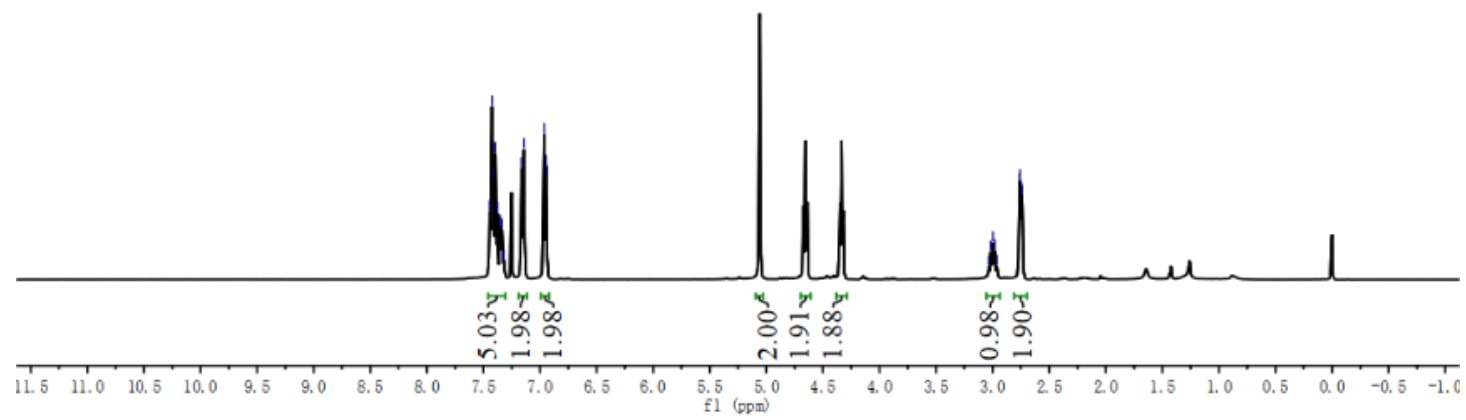

${ }^{13} \mathrm{C}$ NMR (100 MHz, $\mathrm{CDCl}_{3}$ ) spectrum of compound 14 

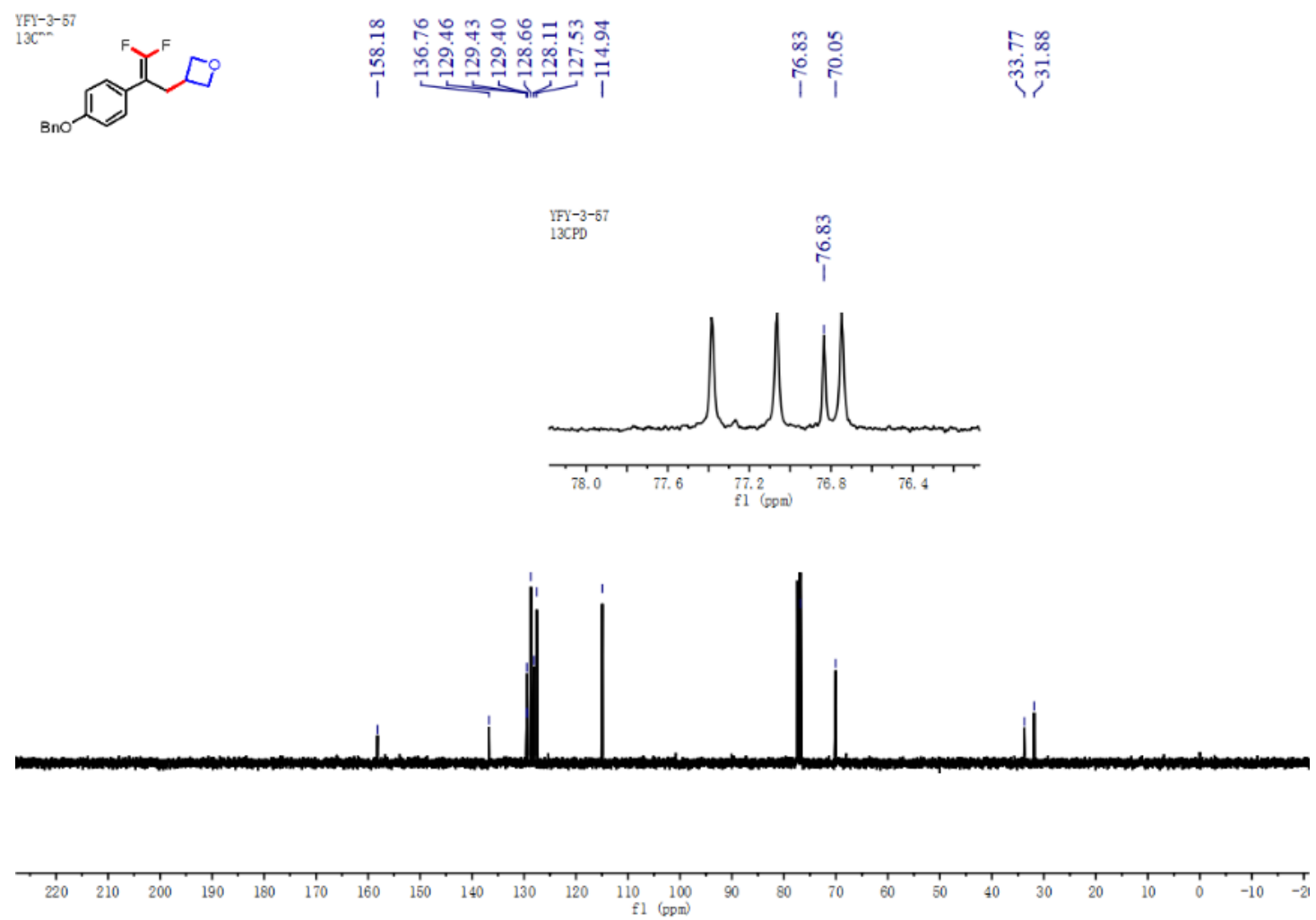

${ }^{19} \mathrm{~F}$ NMR (376 MHz, $\mathrm{CDCl}_{3}$ ) spectrum of compound 14
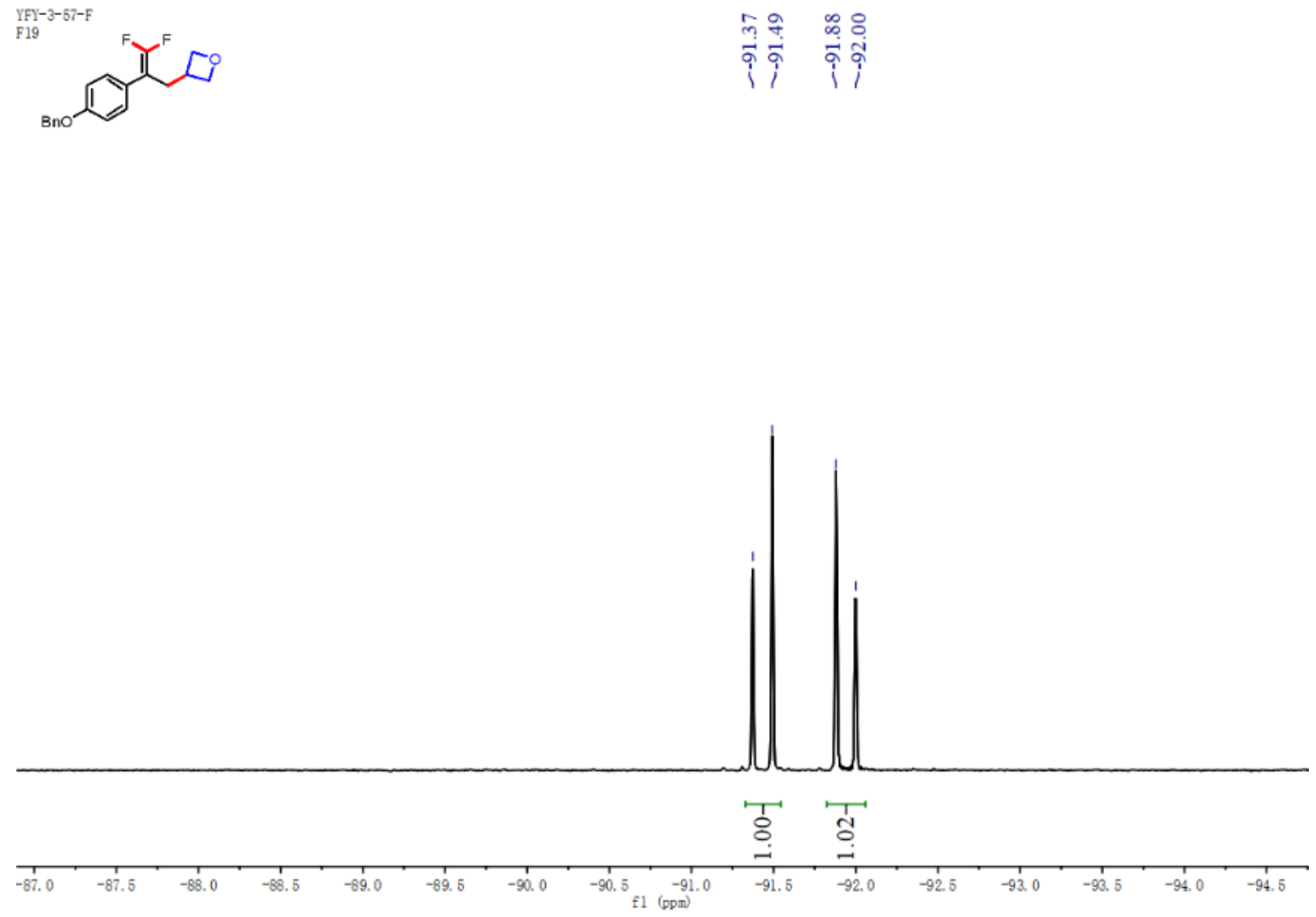

${ }^{1} \mathrm{H}$ NMR (400 MHz, $\mathrm{CDCl}_{3}$ ) spectrum of compound 15 
<smiles>C=[Ge]C</smiles>

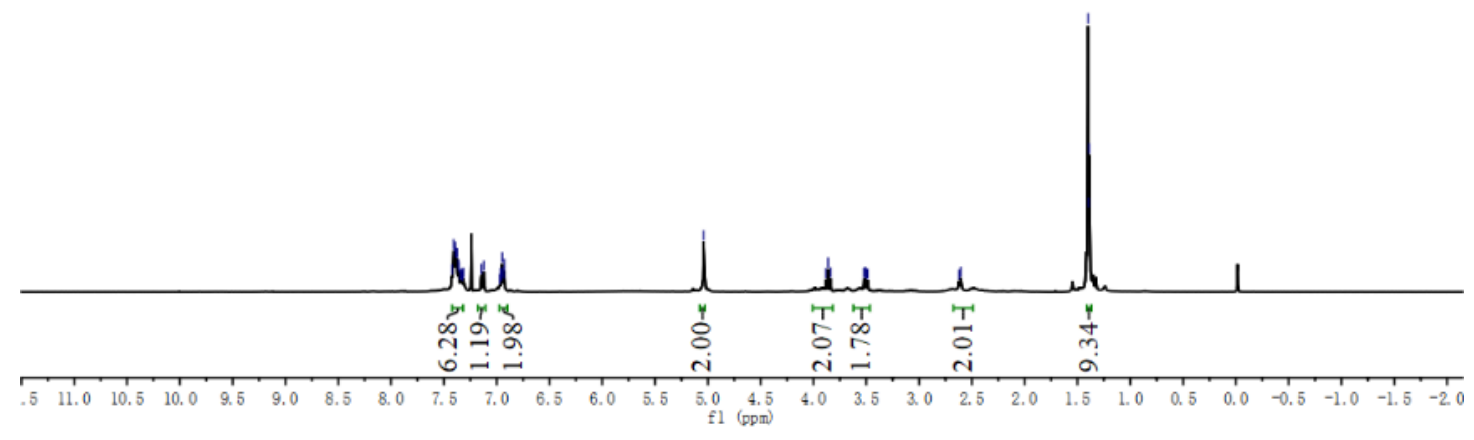

${ }^{13} \mathrm{C}$ NMR (100 MHz, $\mathrm{CDCl}_{3}$ ) spectrum of compound 15
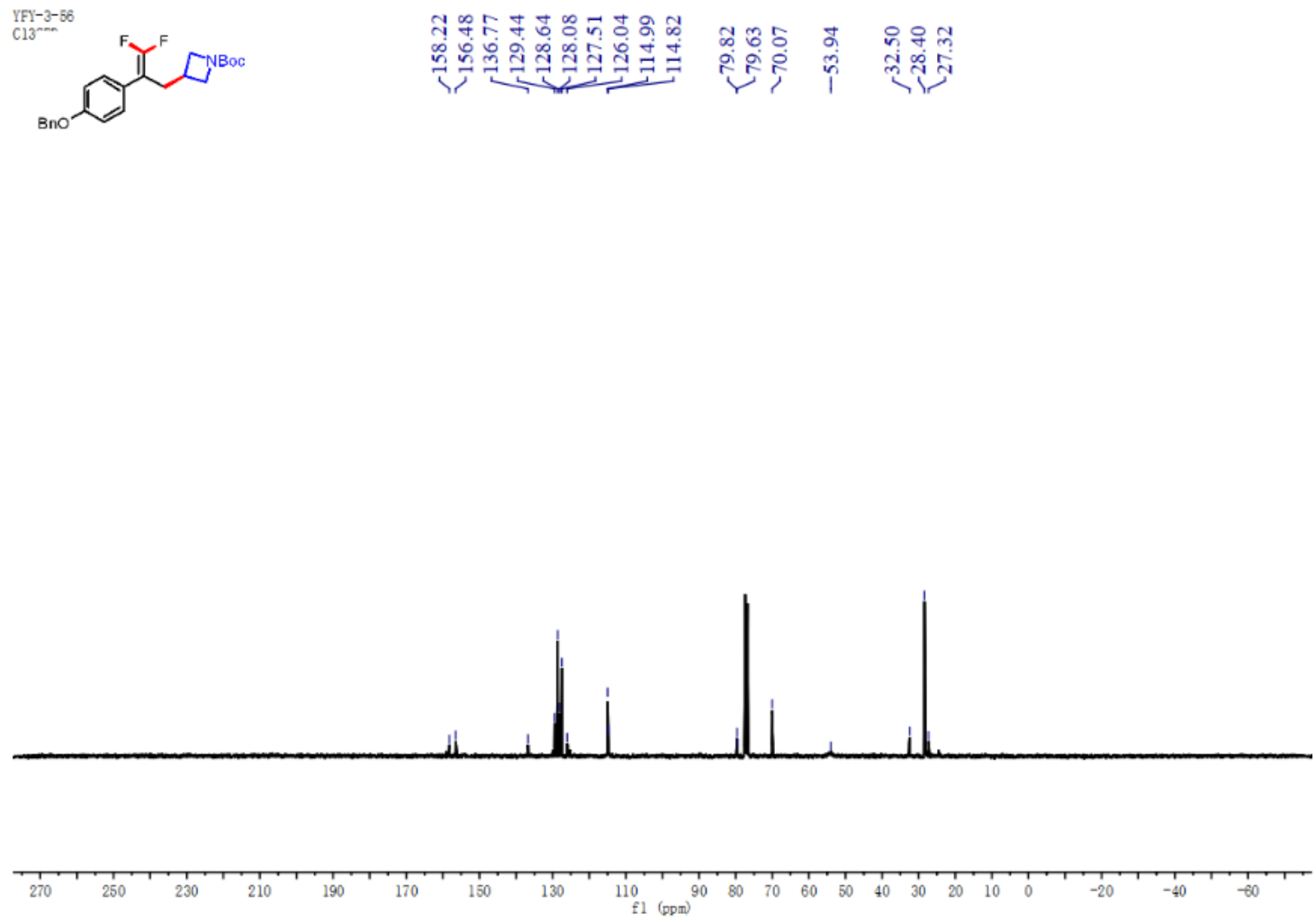

${ }^{19} \mathrm{~F}$ NMR (376 MHz, $\mathrm{CDCl}_{3}$ ) spectrum of compound 15 


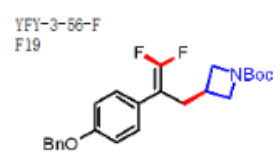

3

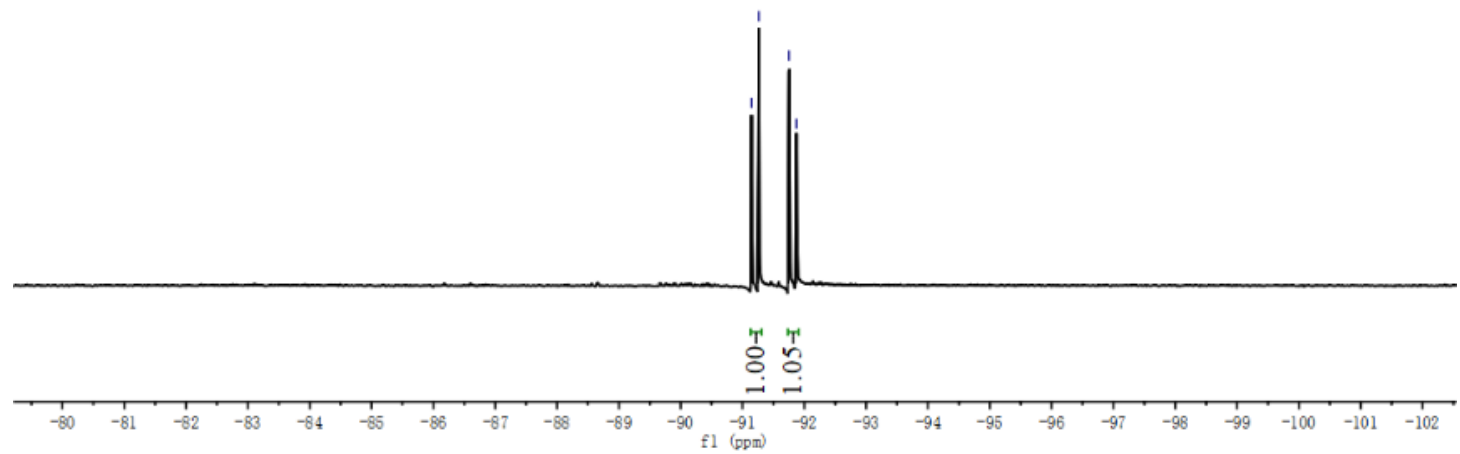

${ }^{1} \mathrm{H}$ NMR (400 MHz, $\mathrm{CDCl}_{3}$ ) spectrum of compound 16

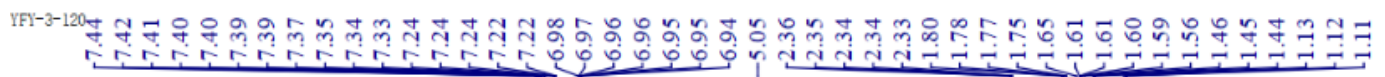
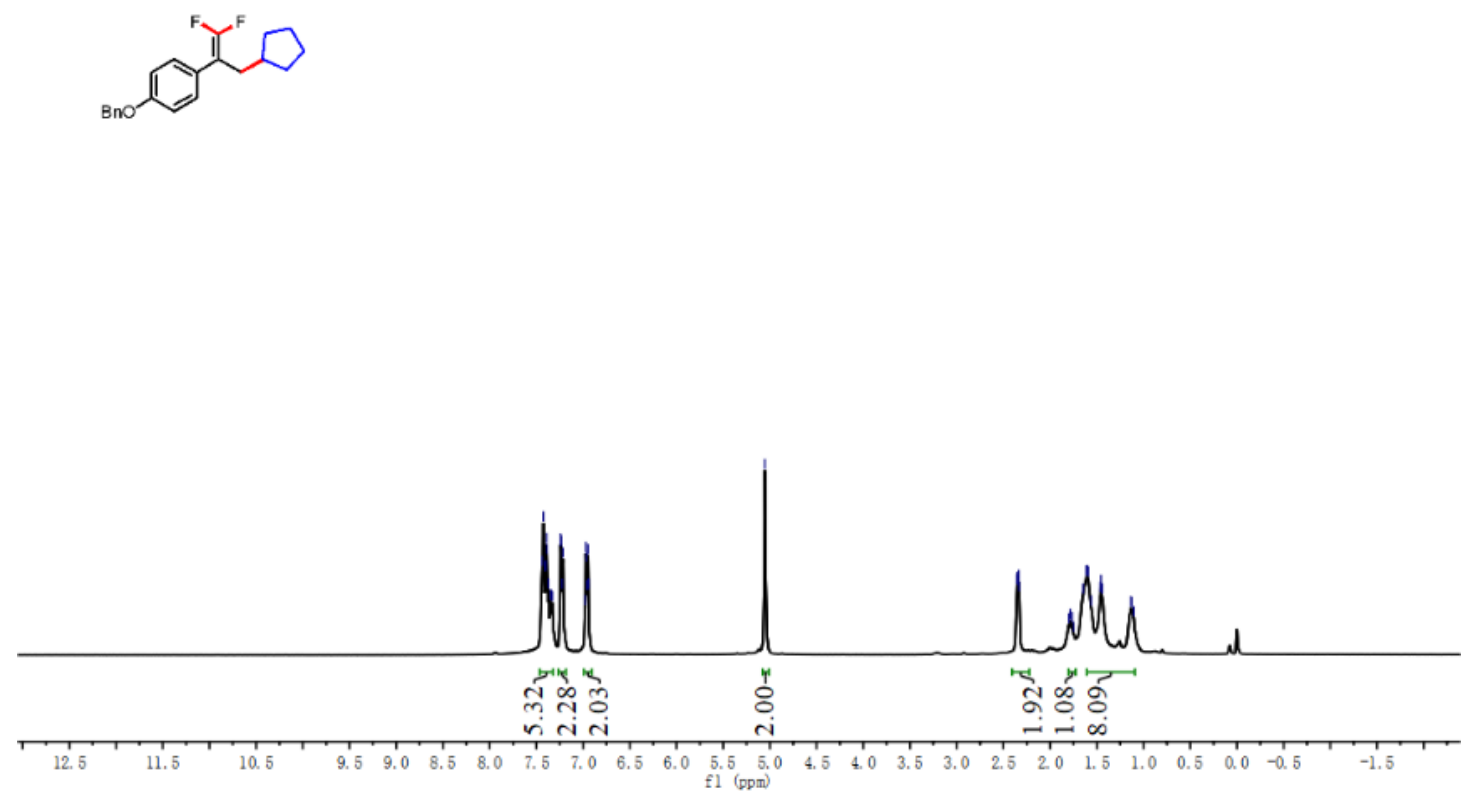

${ }^{13} \mathrm{C}$ NMR (100 MHz, $\mathrm{CDCl}_{3}$ ) spectrum of compound $\mathbf{1 6}$ 

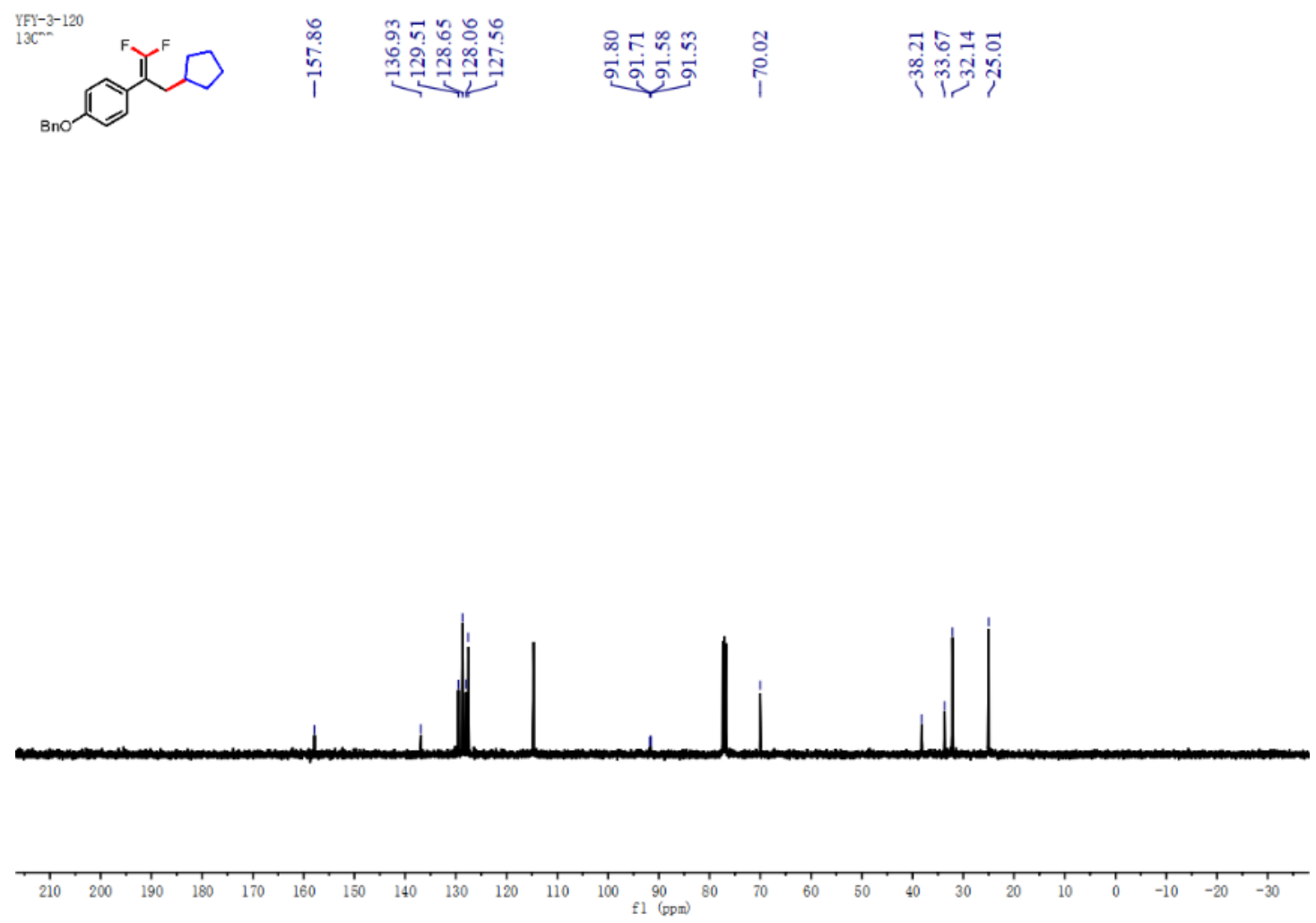

${ }^{19} \mathrm{~F} \mathrm{NMR}\left(376 \mathrm{MHz}, \mathrm{CDCl}_{3}\right.$ ) spectrum of compound 16

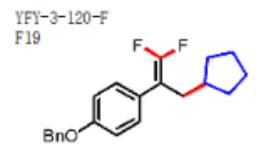

क००ने ले

simi
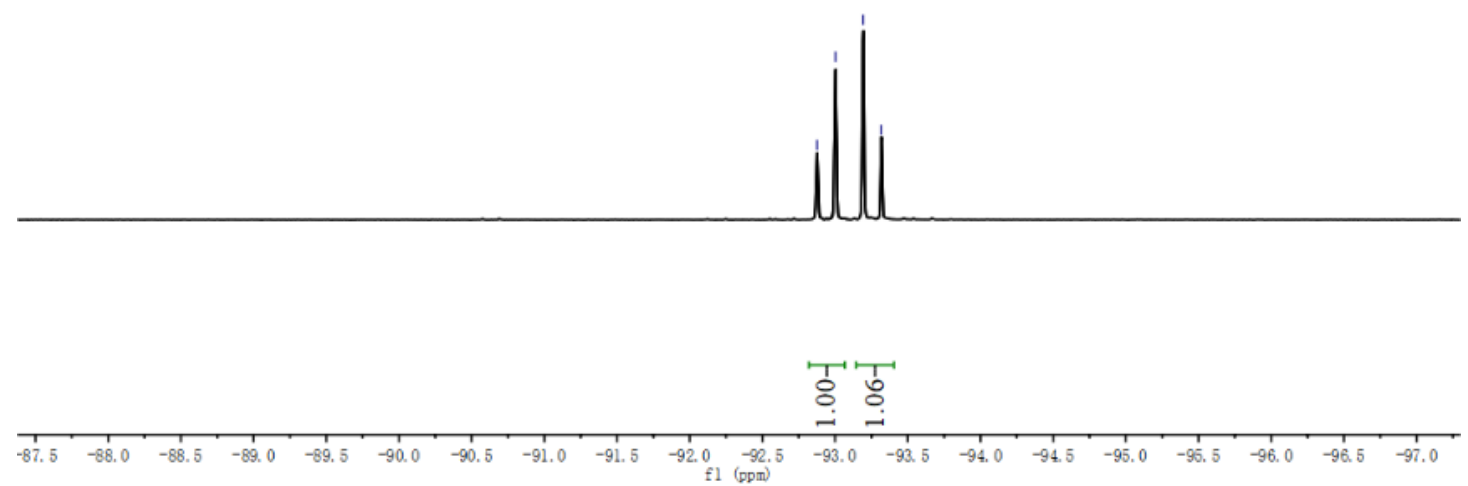

${ }^{1} \mathrm{H}$ NMR (400 MHz, $\mathrm{CDCl}_{3}$ ) spectrum of compound $\mathbf{1 7}$ 

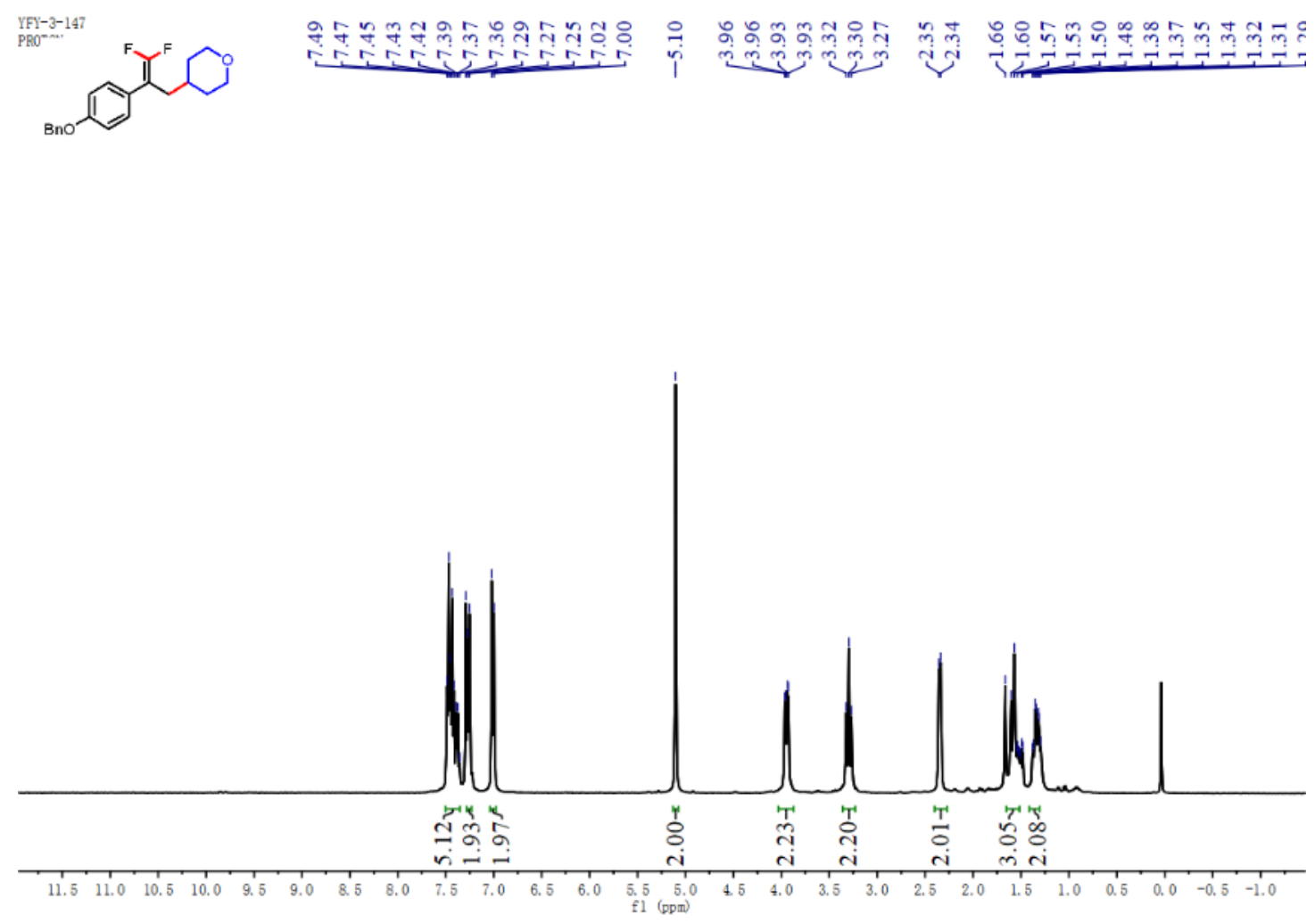

${ }^{13} \mathrm{C}$ NMR (100 MHz, $\mathrm{CDCl}_{3}$ ) spectrum of compound 17

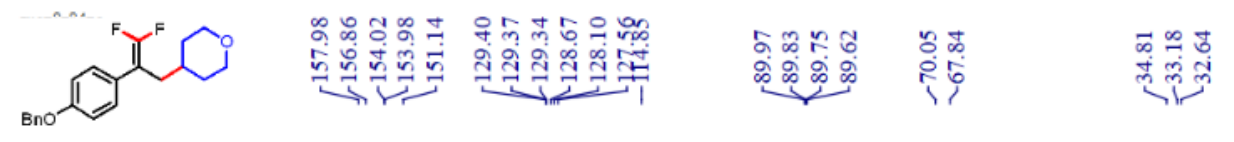
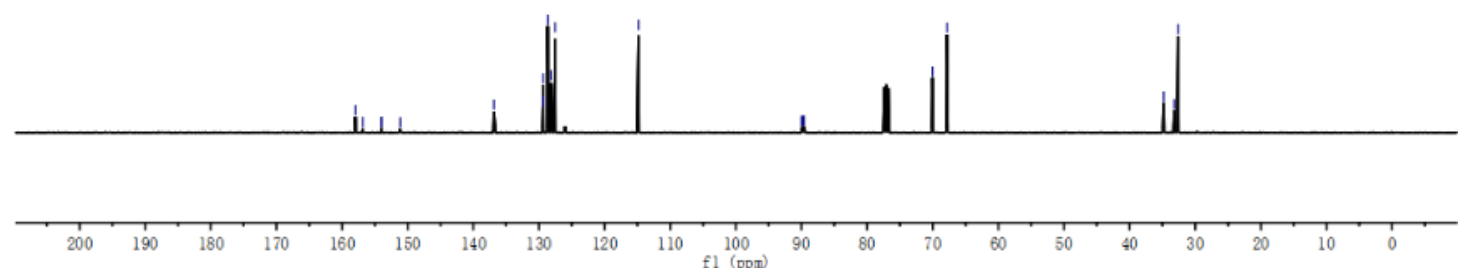

${ }^{19} \mathrm{~F} \mathrm{NMR}\left(376 \mathrm{MHz}, \mathrm{CDCl}_{3}\right.$ ) spectrum of compound 17 

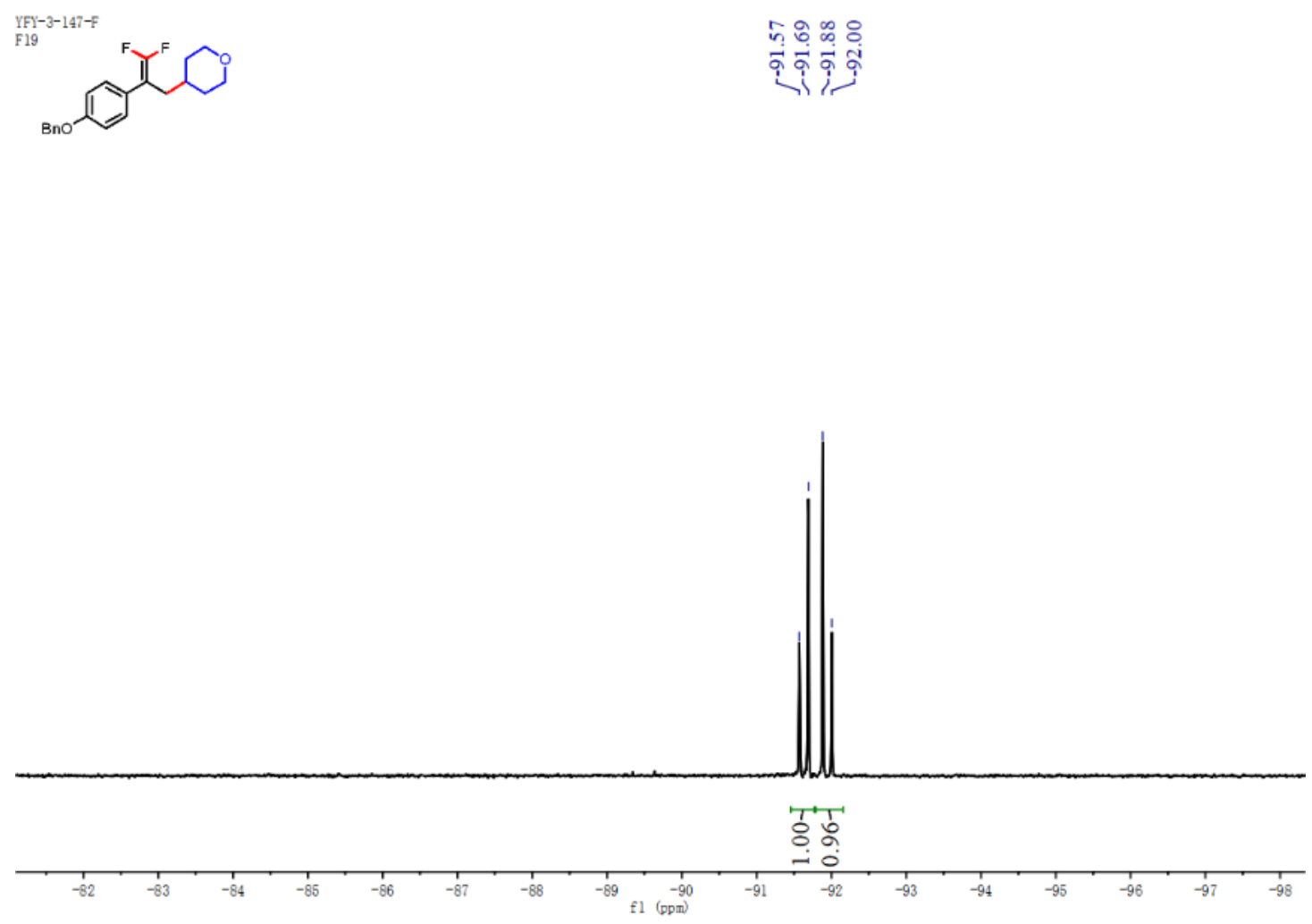

${ }^{1} \mathrm{H}$ NMR (400 MHz, $\mathrm{CDCl}_{3}$ ) spectrum of compound 18
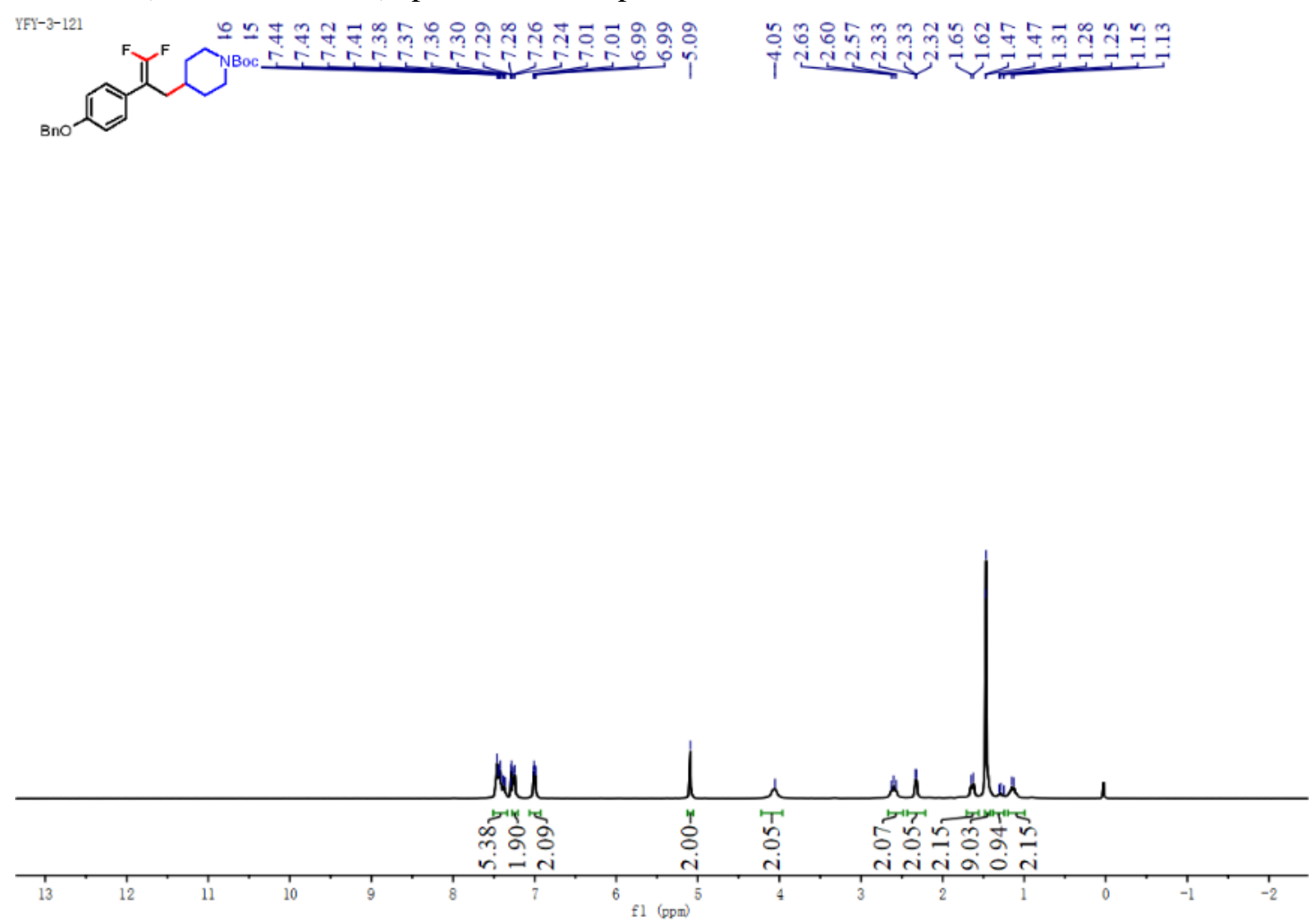

${ }^{13} \mathrm{C}$ NMR (100 MHz, $\mathrm{CDCl}_{3}$ ) spectrum of compound $\mathbf{1 8}$ 

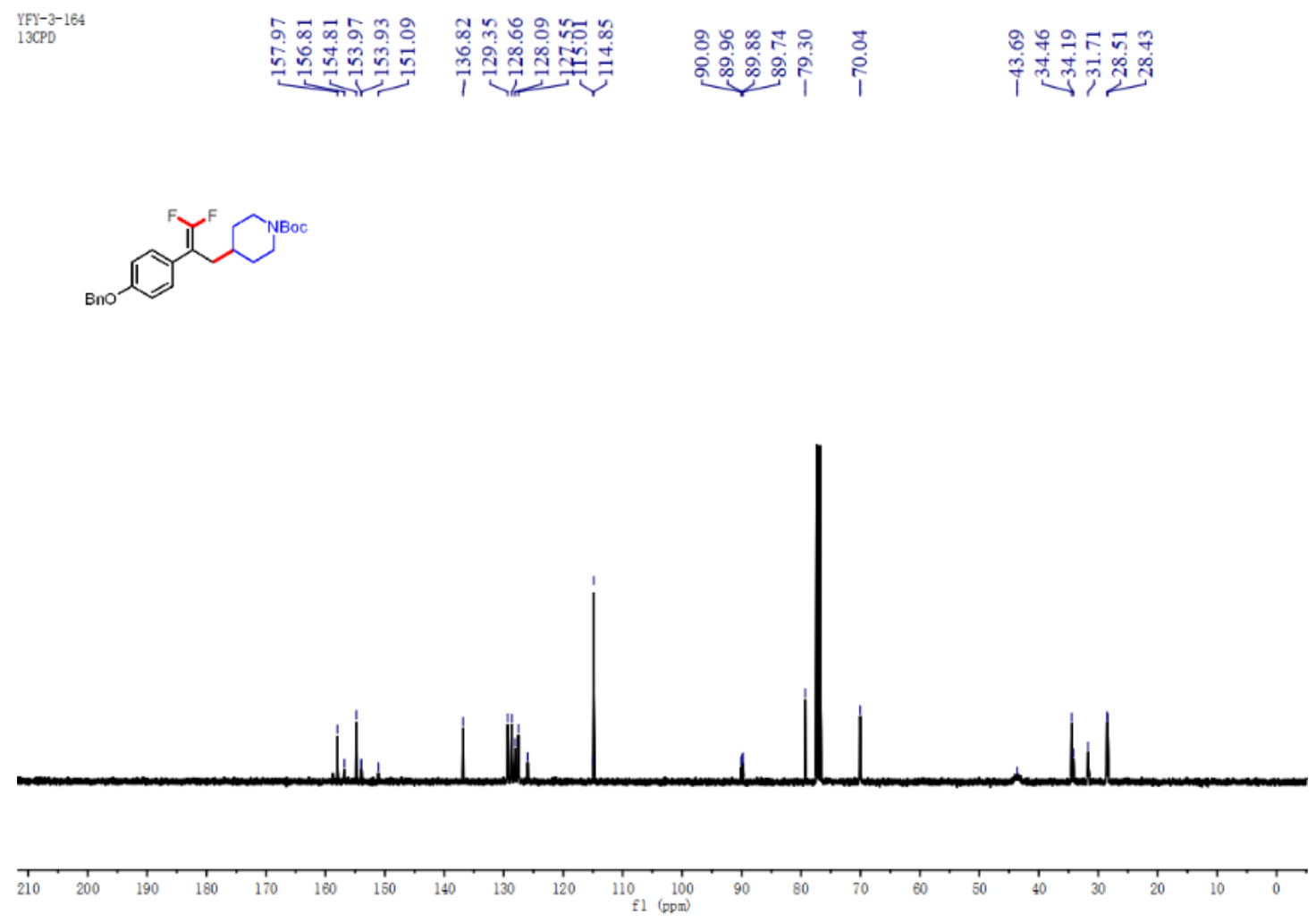

${ }^{19} \mathrm{~F} \mathrm{NMR}\left(376 \mathrm{MHz}, \mathrm{CDCl}_{3}\right)$ spectrum of compound 18

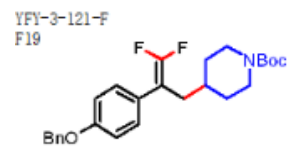

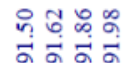

a

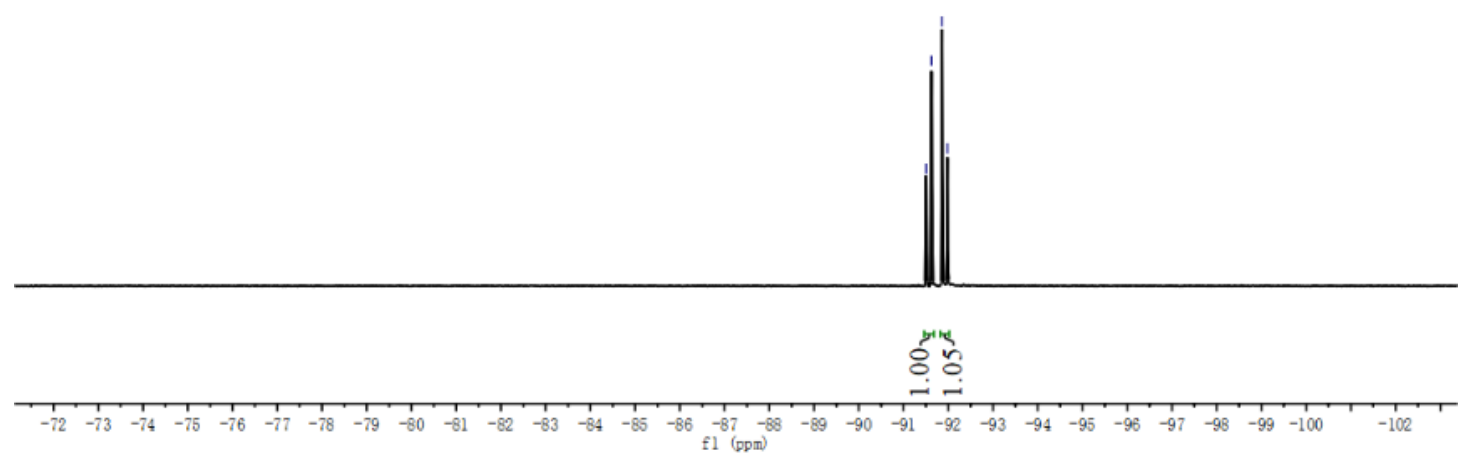

${ }^{1} \mathrm{H}$ NMR (400 MHz, $\mathrm{CDCl}_{3}$ ) spectrum of compound 19 

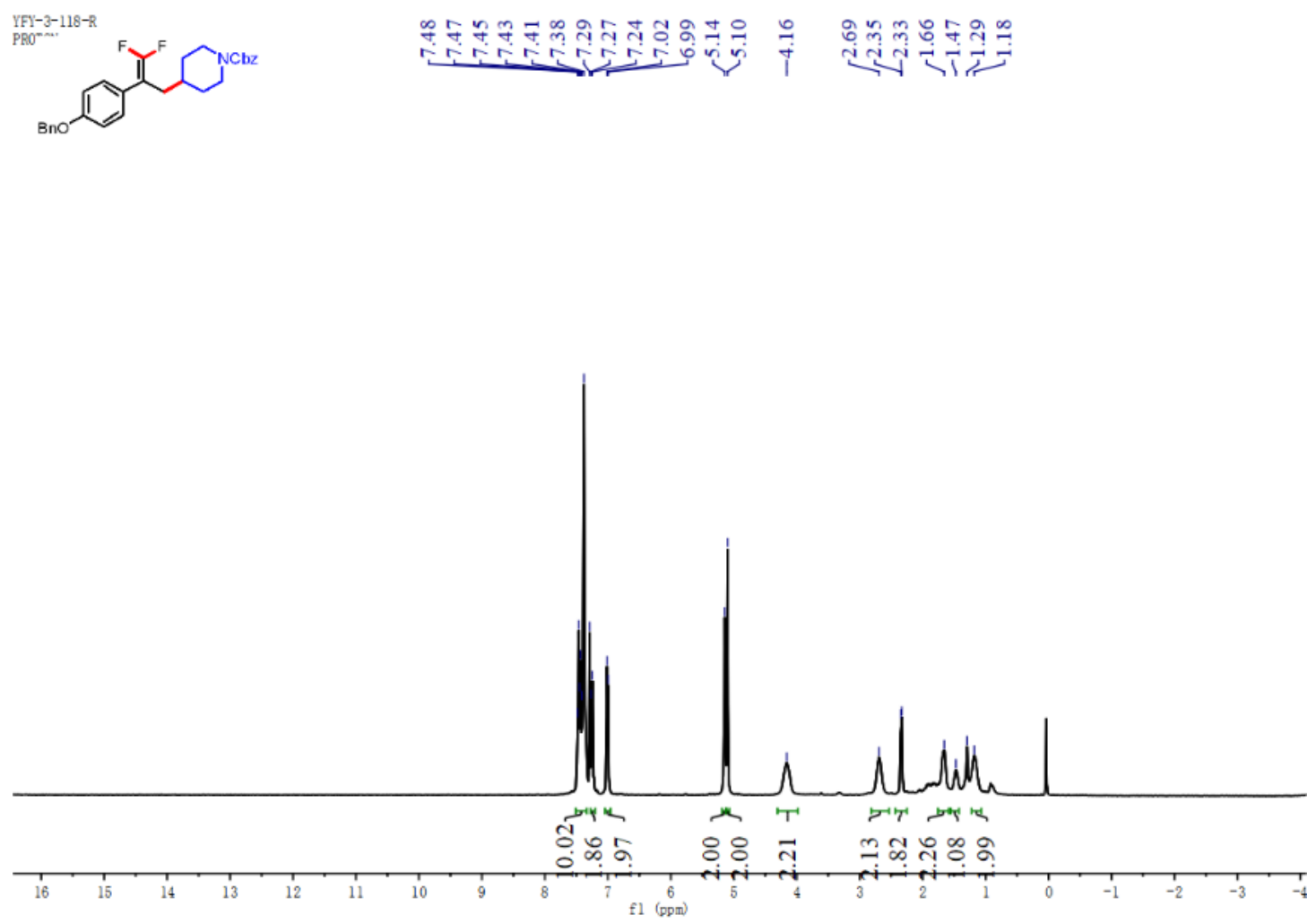

${ }^{13} \mathrm{C}$ NMR (100 MHz, $\mathrm{CDCl}_{3}$ ) spectrum of compound 19

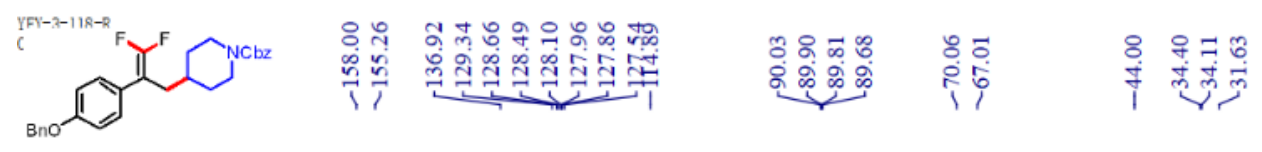
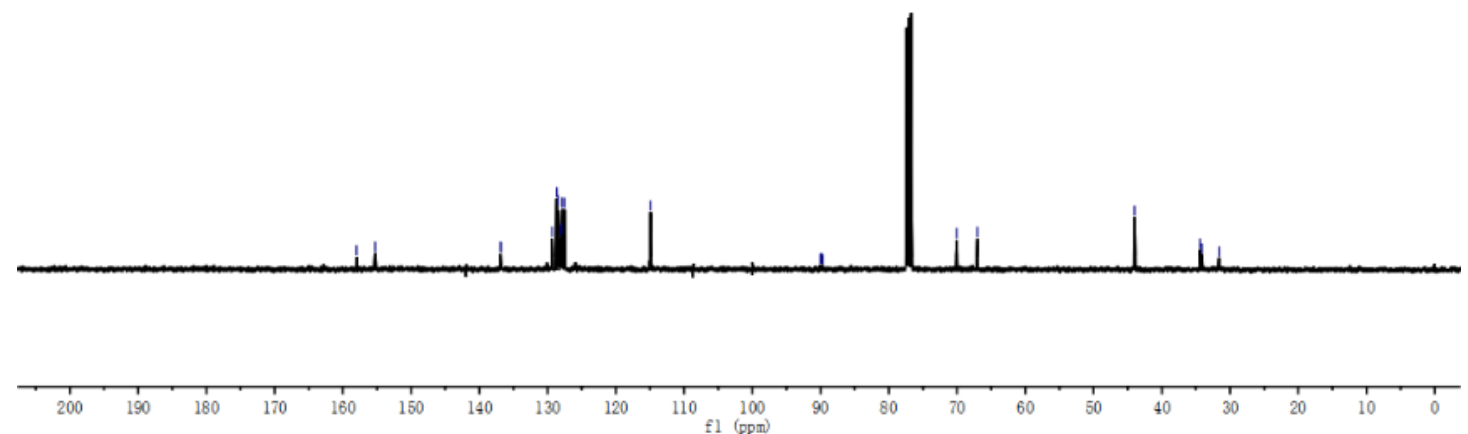

${ }^{19} \mathrm{~F}$ NMR (376 MHz, $\mathrm{CDCl}_{3}$ ) spectrum of compound 19 

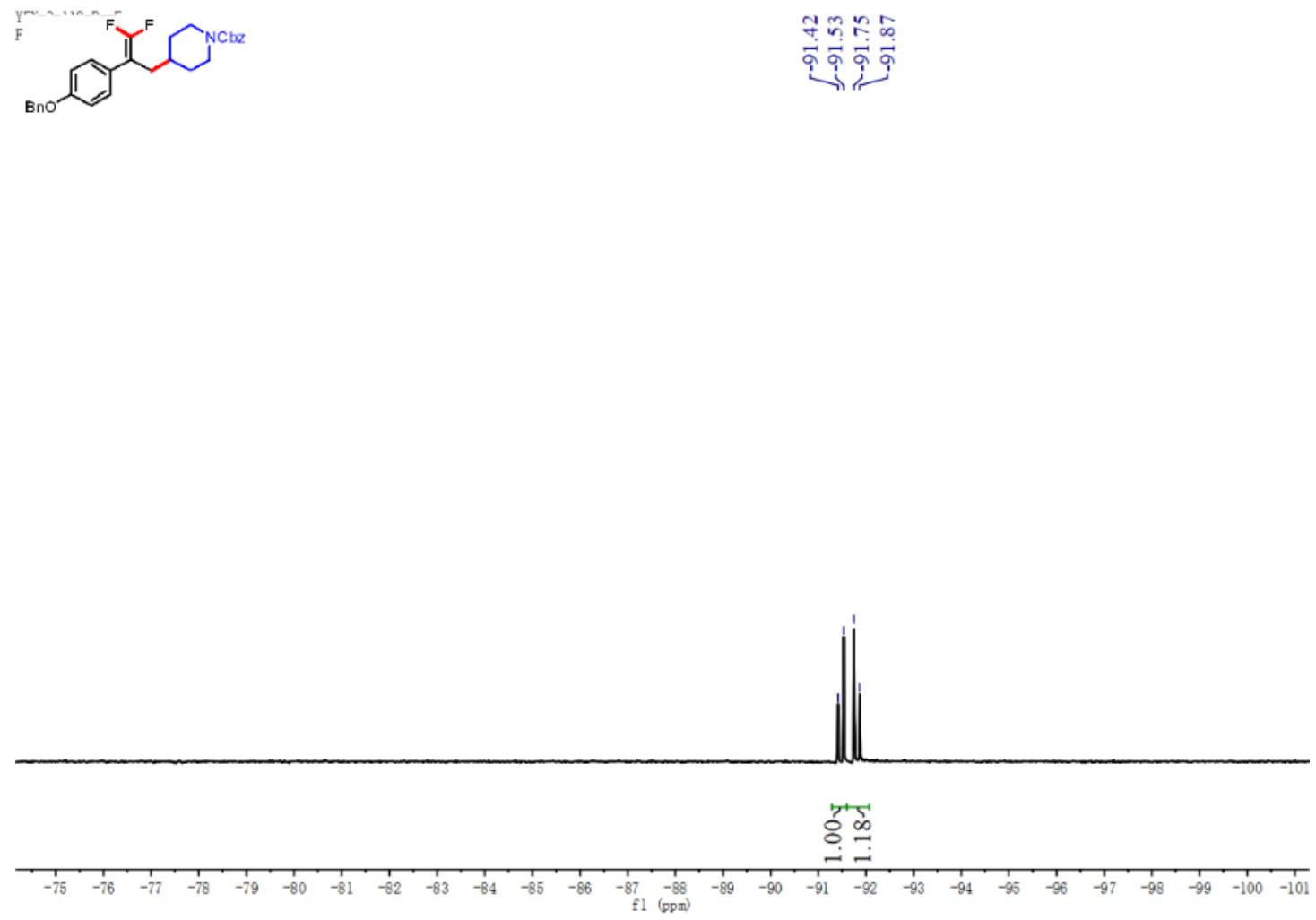

${ }^{1} \mathrm{H}$ NMR (400 MHz, $\mathrm{CDCl}_{3}$ ) spectrum of compound 20
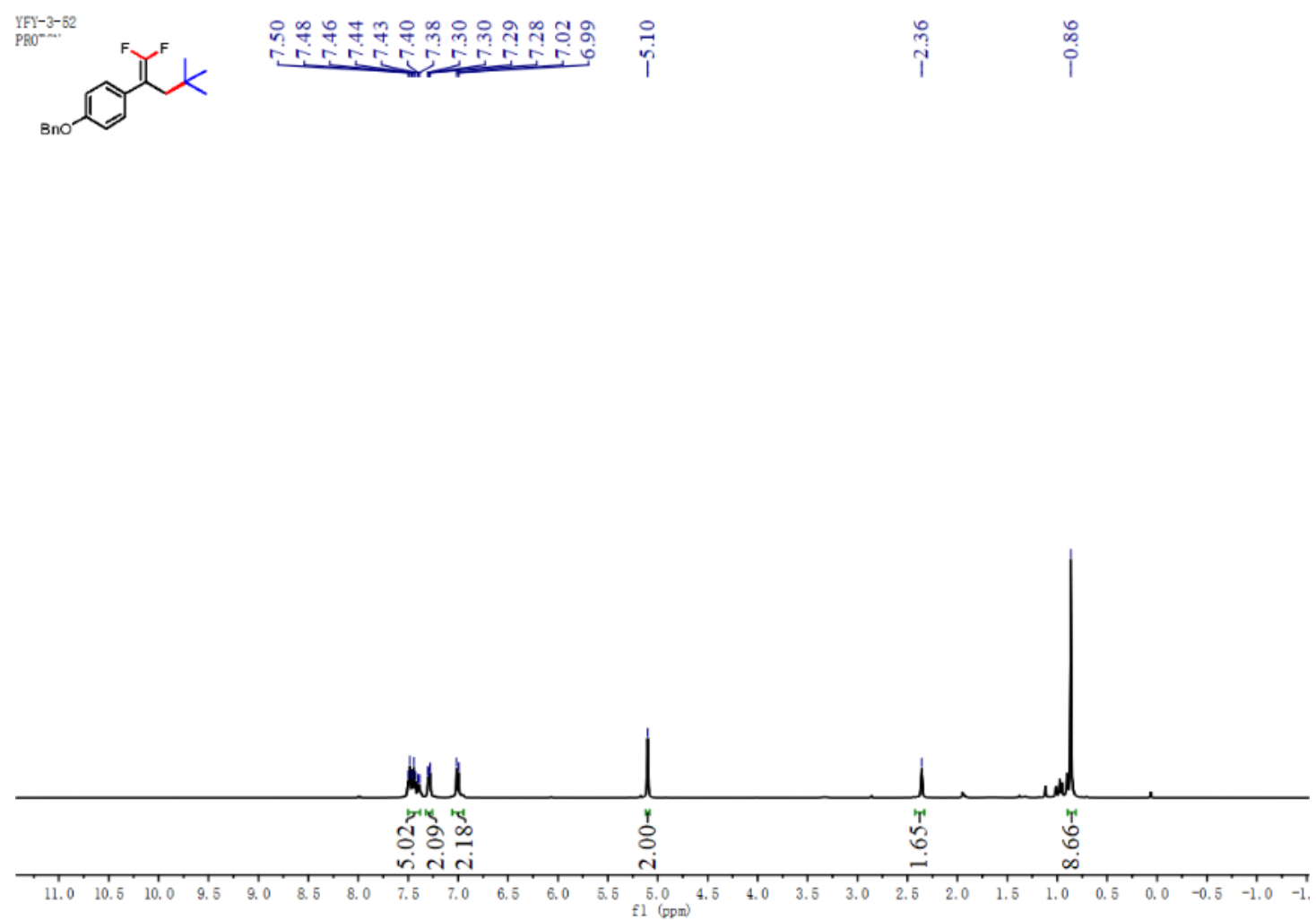

${ }^{13} \mathrm{C}$ NMR (100 MHz, $\mathrm{CDCl}_{3}$ ) spectrum of compound 20 

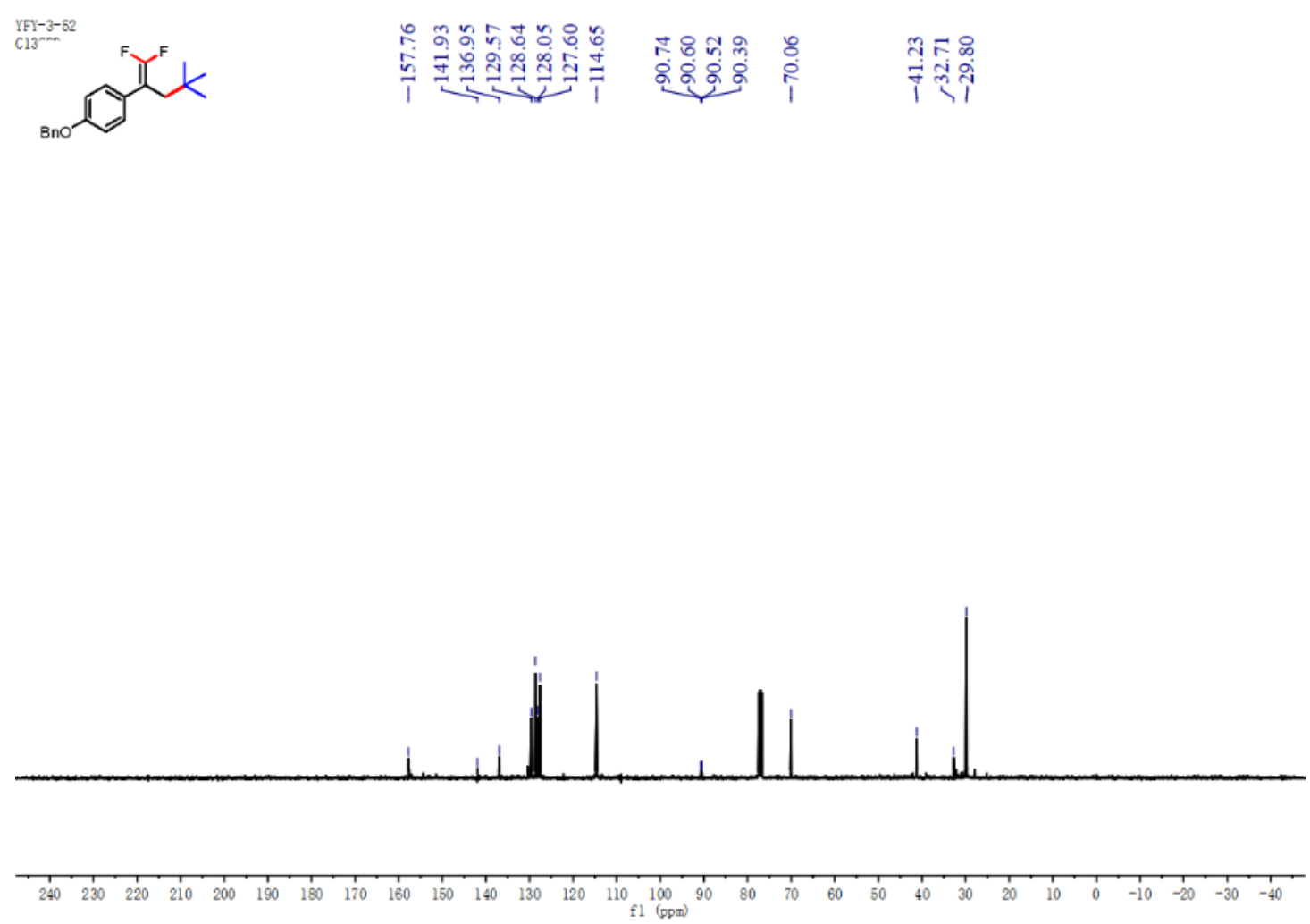

${ }^{19} \mathrm{~F}$ NMR (376 MHz, $\mathrm{CDCl}_{3}$ ) spectrum of compound 20
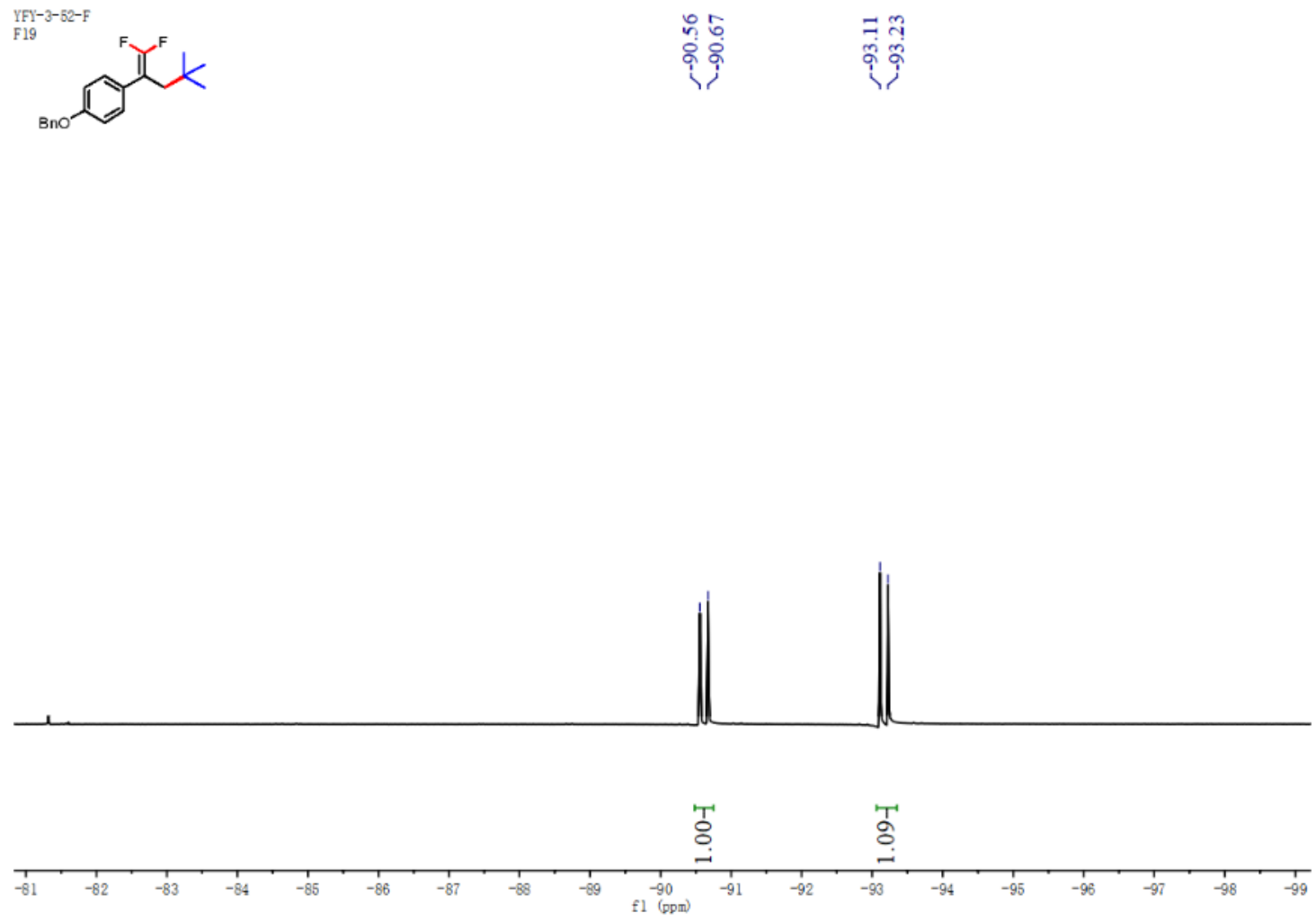

${ }^{1} \mathrm{H}$ NMR (400 MHz, $\mathrm{CDCl}_{3}$ ) spectrum of compound 21 

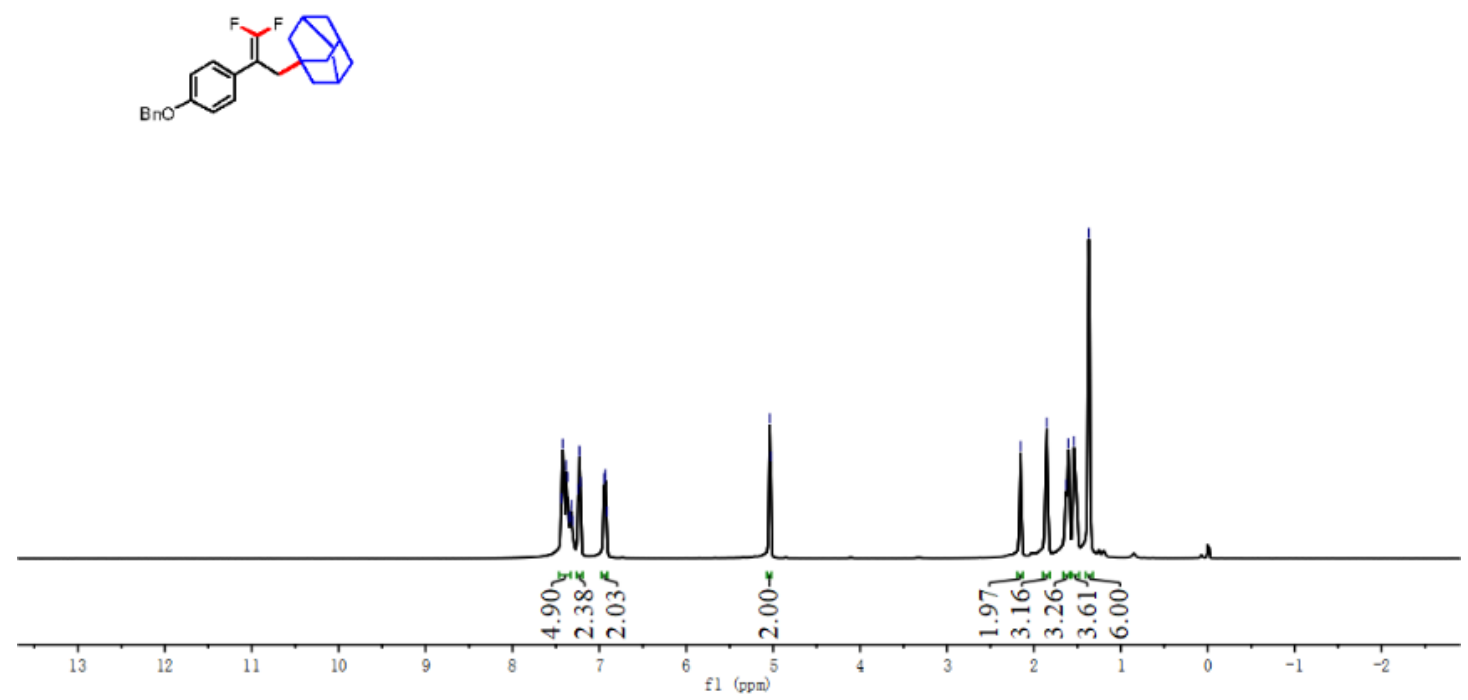

${ }^{13} \mathrm{C}$ NMR (100 MHz, $\mathrm{CDCl}_{3}$ ) spectrum of compound 21

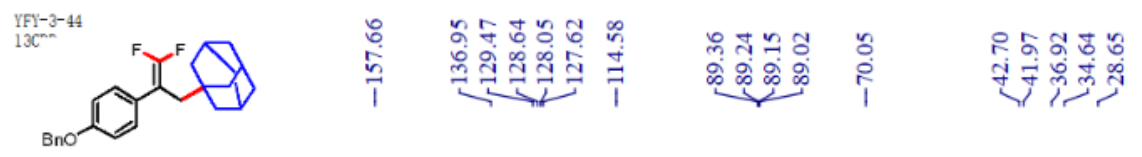

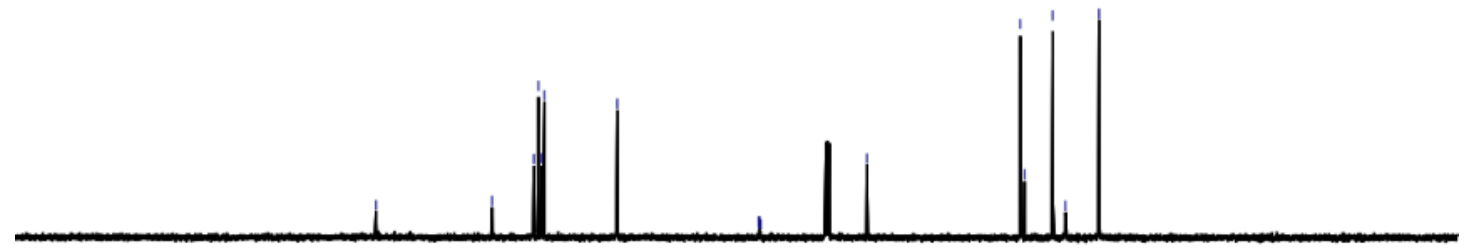

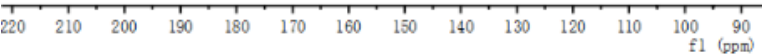

${ }^{19} \mathrm{~F}$ NMR (376 MHz, $\mathrm{CDCl}_{3}$ ) spectrum of compound 21 

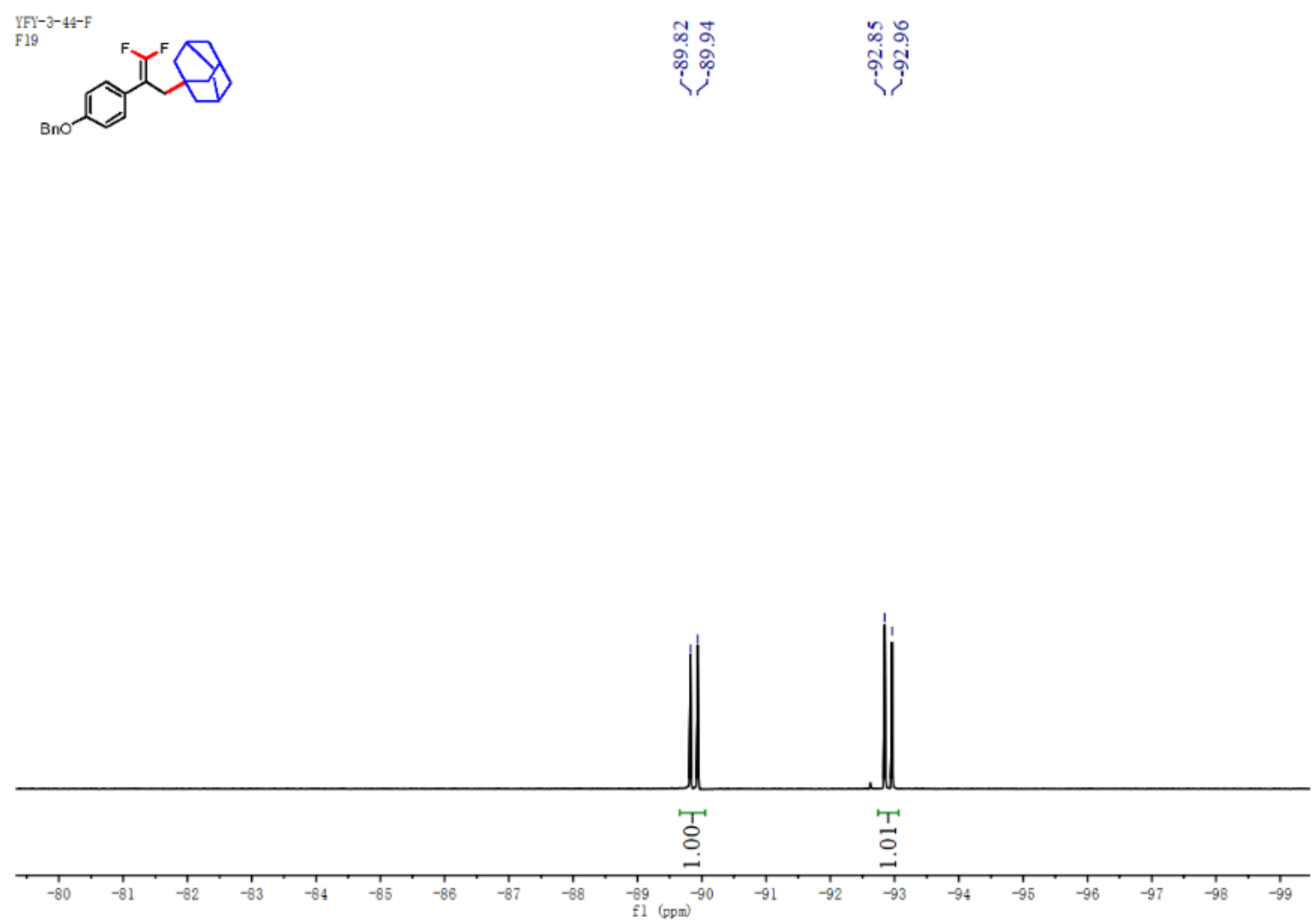

${ }^{1} \mathrm{H}$ NMR (400 MHz, $\mathrm{CDCl}_{3}$ ) spectrum of compound 22
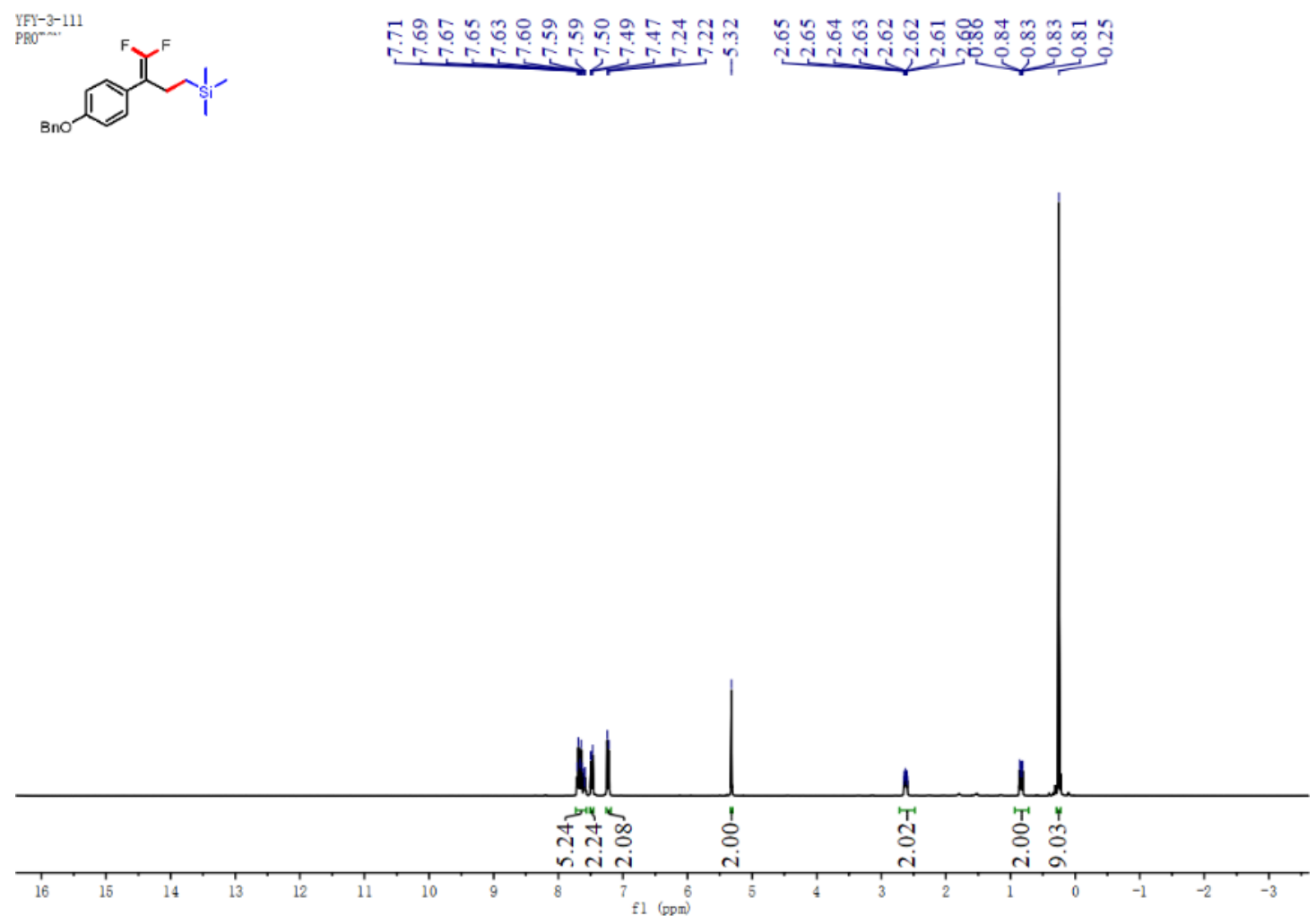

${ }^{13} \mathrm{C}$ NMR (100 MHz, $\mathrm{CDCl}_{3}$ ) spectrum of compound 22 

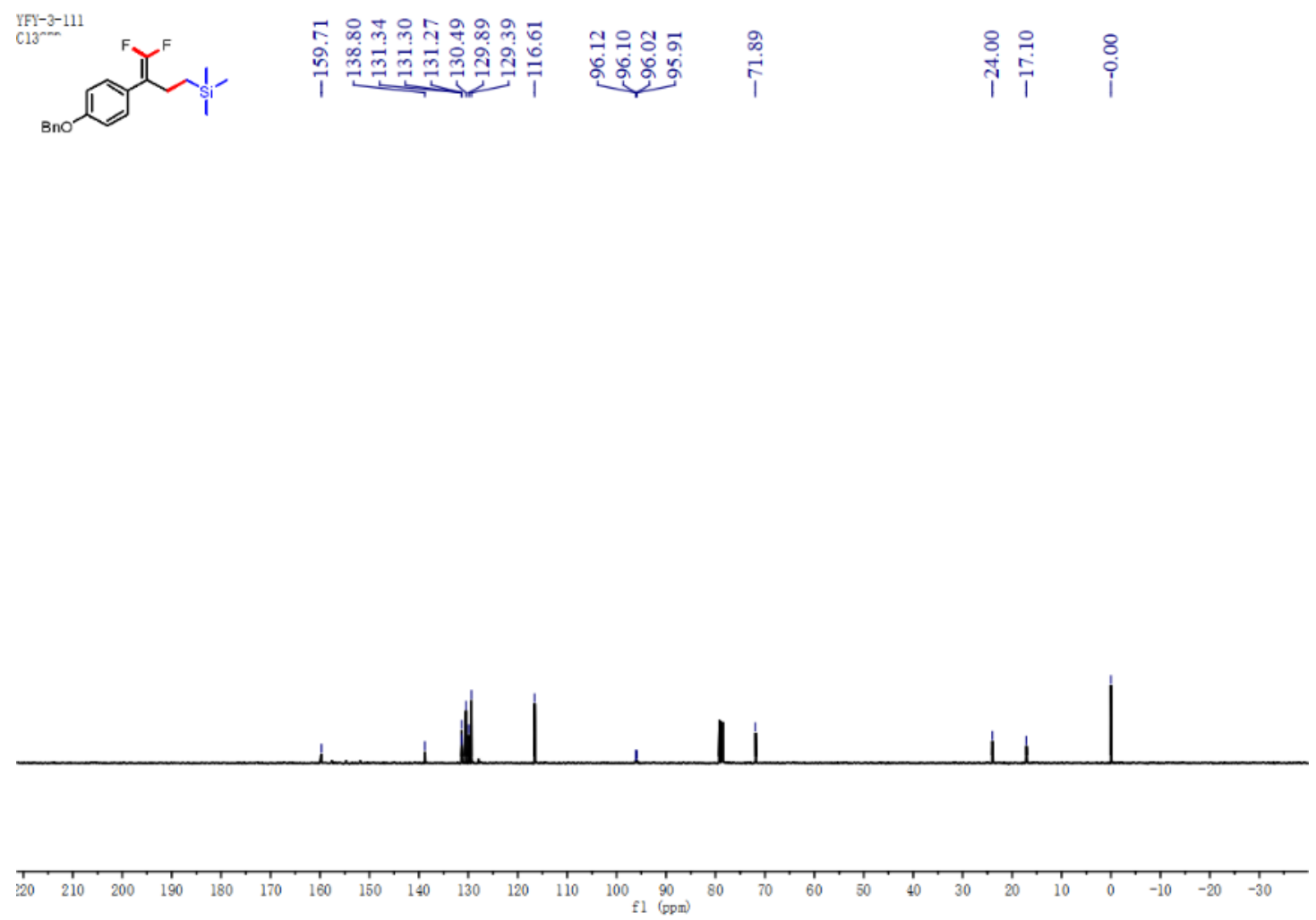

${ }^{19} \mathrm{~F}$ NMR (376 MHz, $\mathrm{CDCl}_{3}$ ) spectrum of compound 22

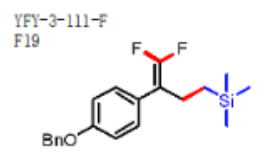

궁ำ

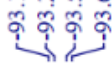

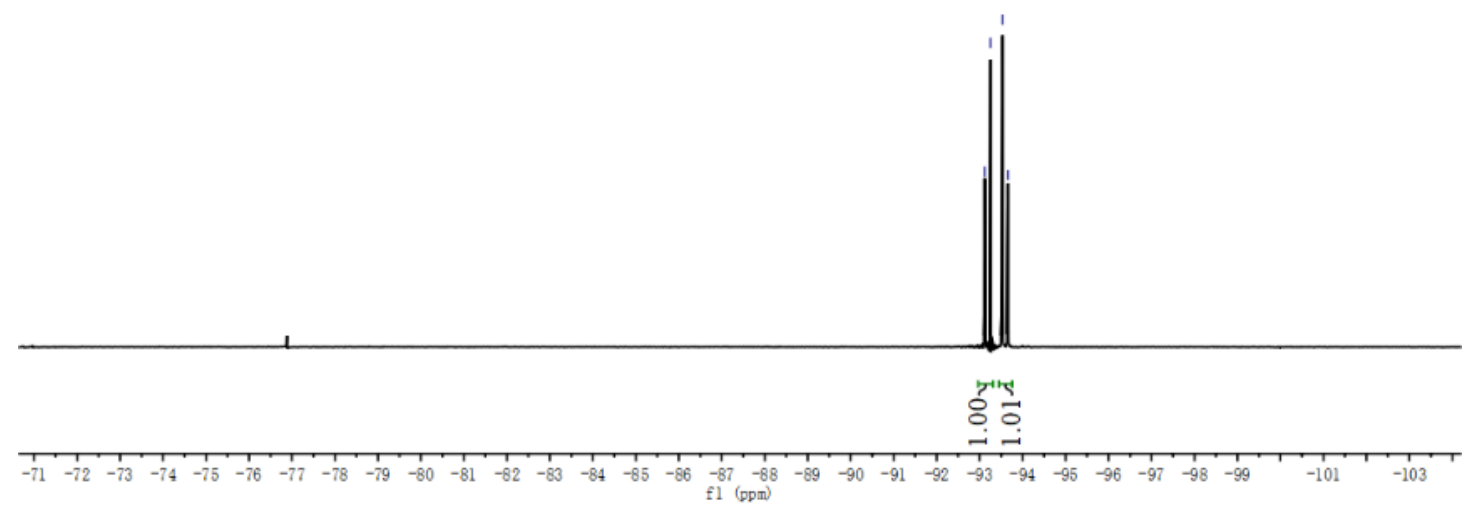

${ }^{1} \mathrm{H}$ NMR (400 MHz, $\mathrm{CDCl}_{3}$ ) spectrum of compound 23 

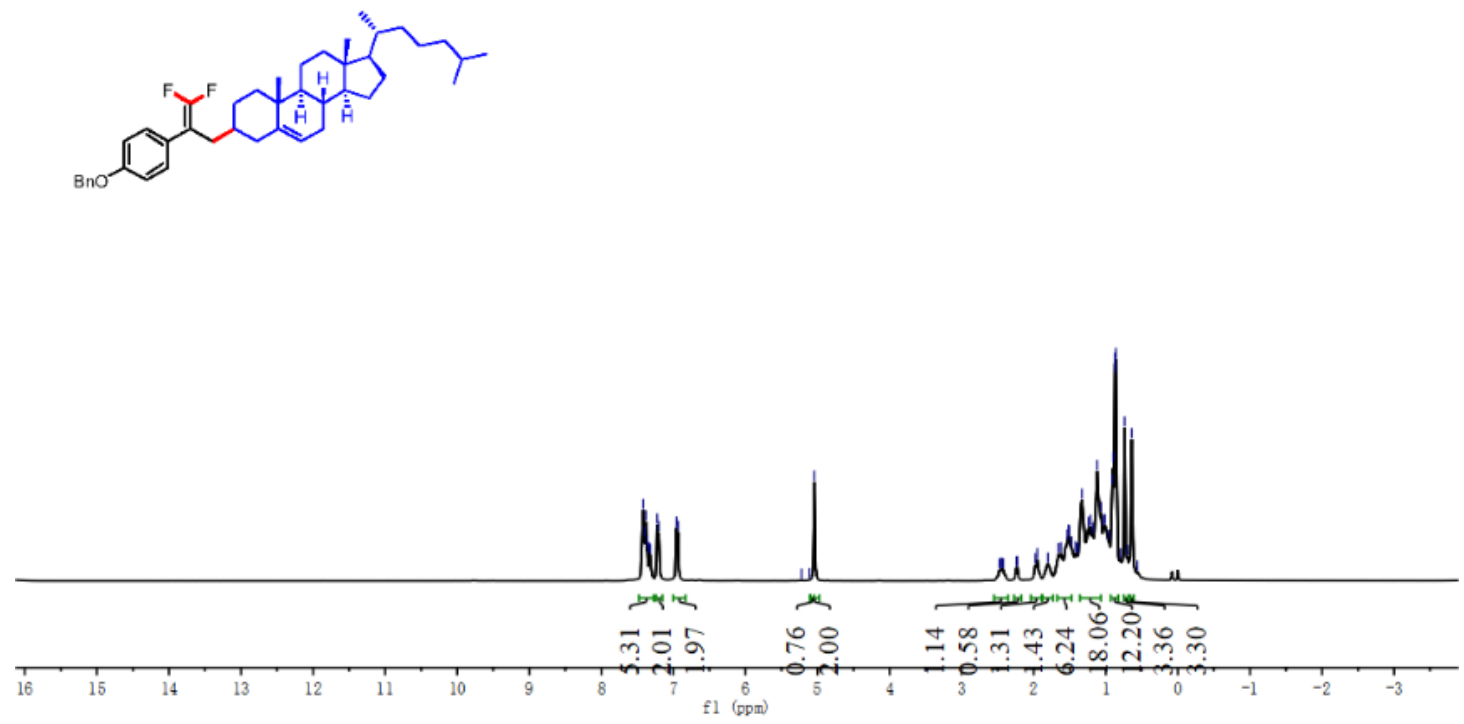

${ }^{13} \mathrm{C}$ NMR (100 MHz, $\mathrm{CDCl}_{3}$ ) spectrum of compound 23

perp

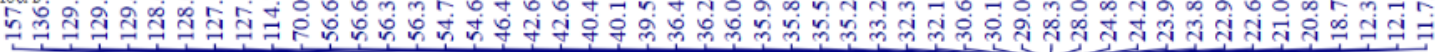
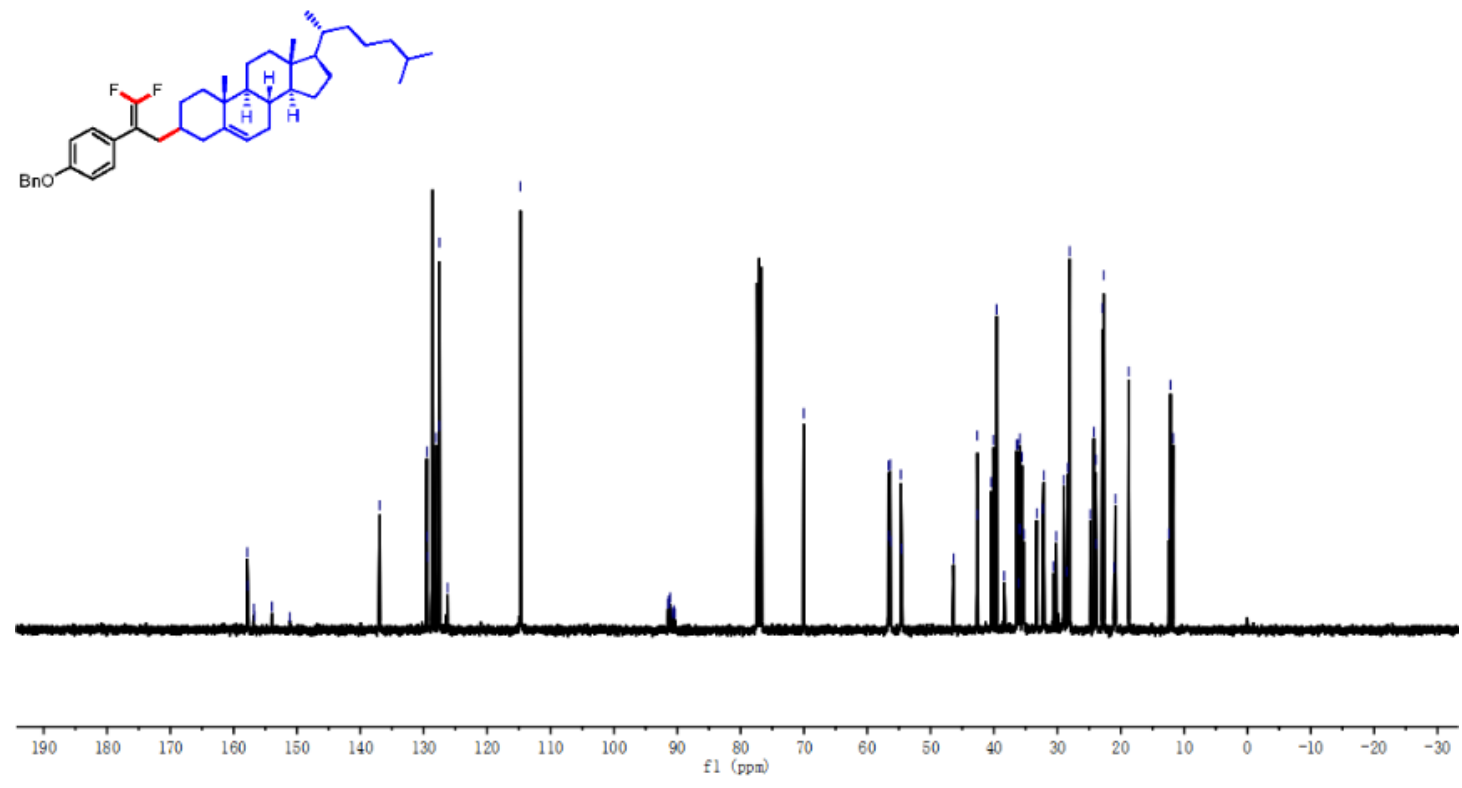

${ }^{19} \mathrm{~F}$ NMR (376 MHz, $\mathrm{CDCl}_{3}$ ) spectrum of compound $\mathbf{2 3}$ 


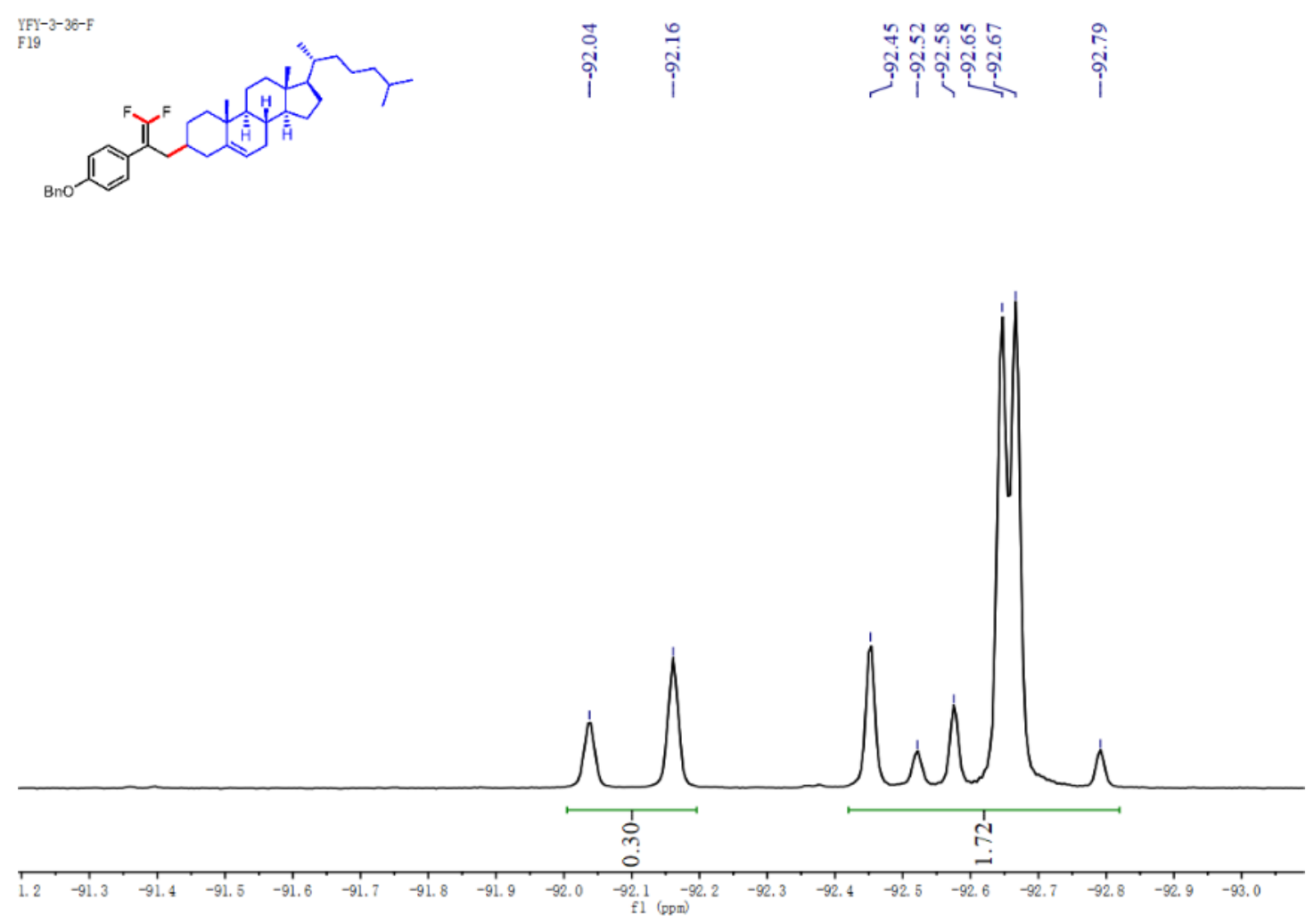

${ }^{1} \mathrm{H}$ NMR (400 MHz, $\mathrm{CDCl}_{3}$ ) spectrum of compound 24

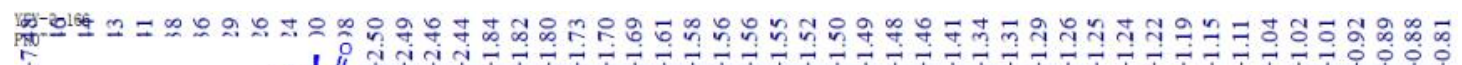

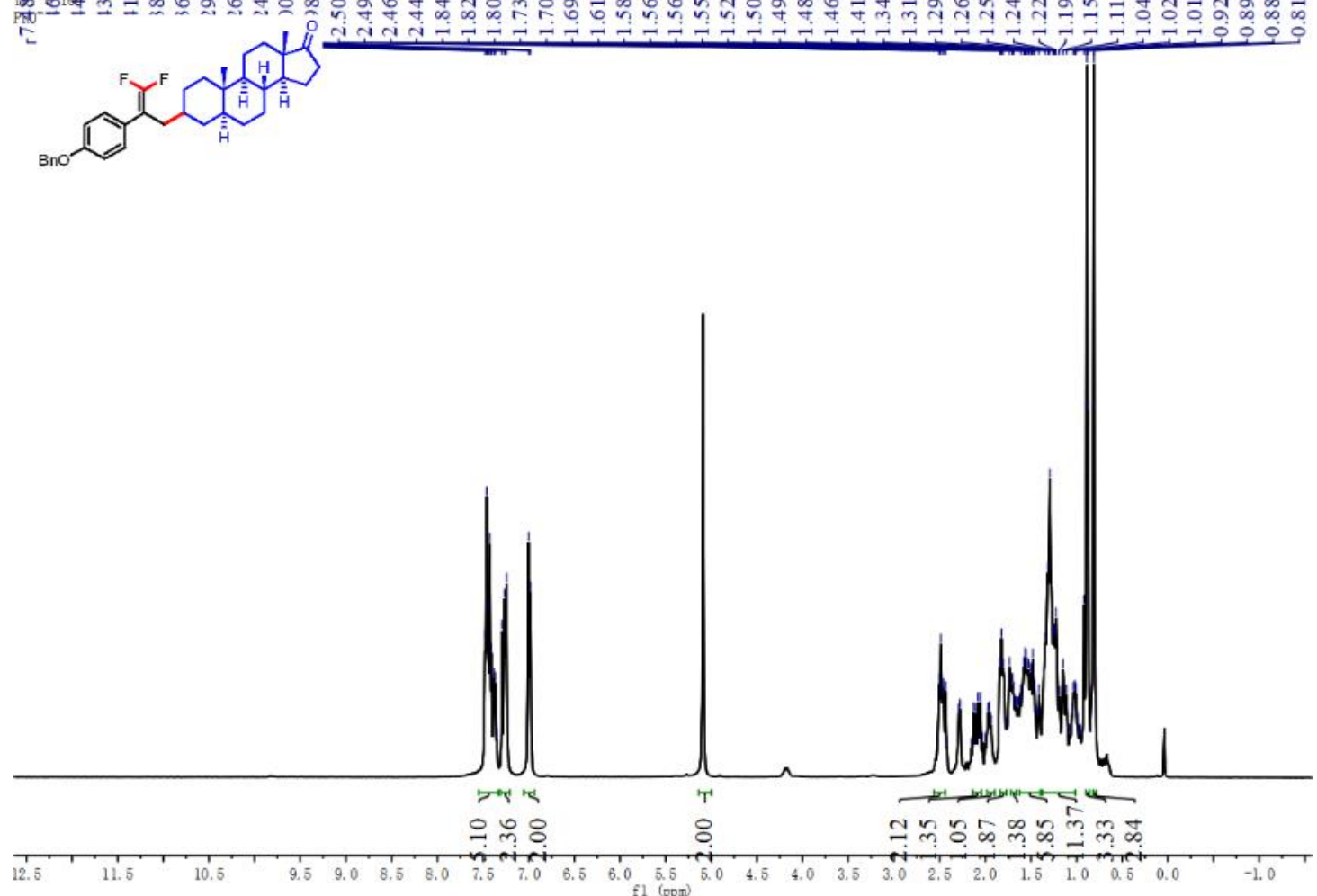

${ }^{13} \mathrm{C}$ NMR (100 MHz, $\left.\mathrm{CDCl}_{3}\right)$ spectrum of compound $\mathbf{2 4}$ 

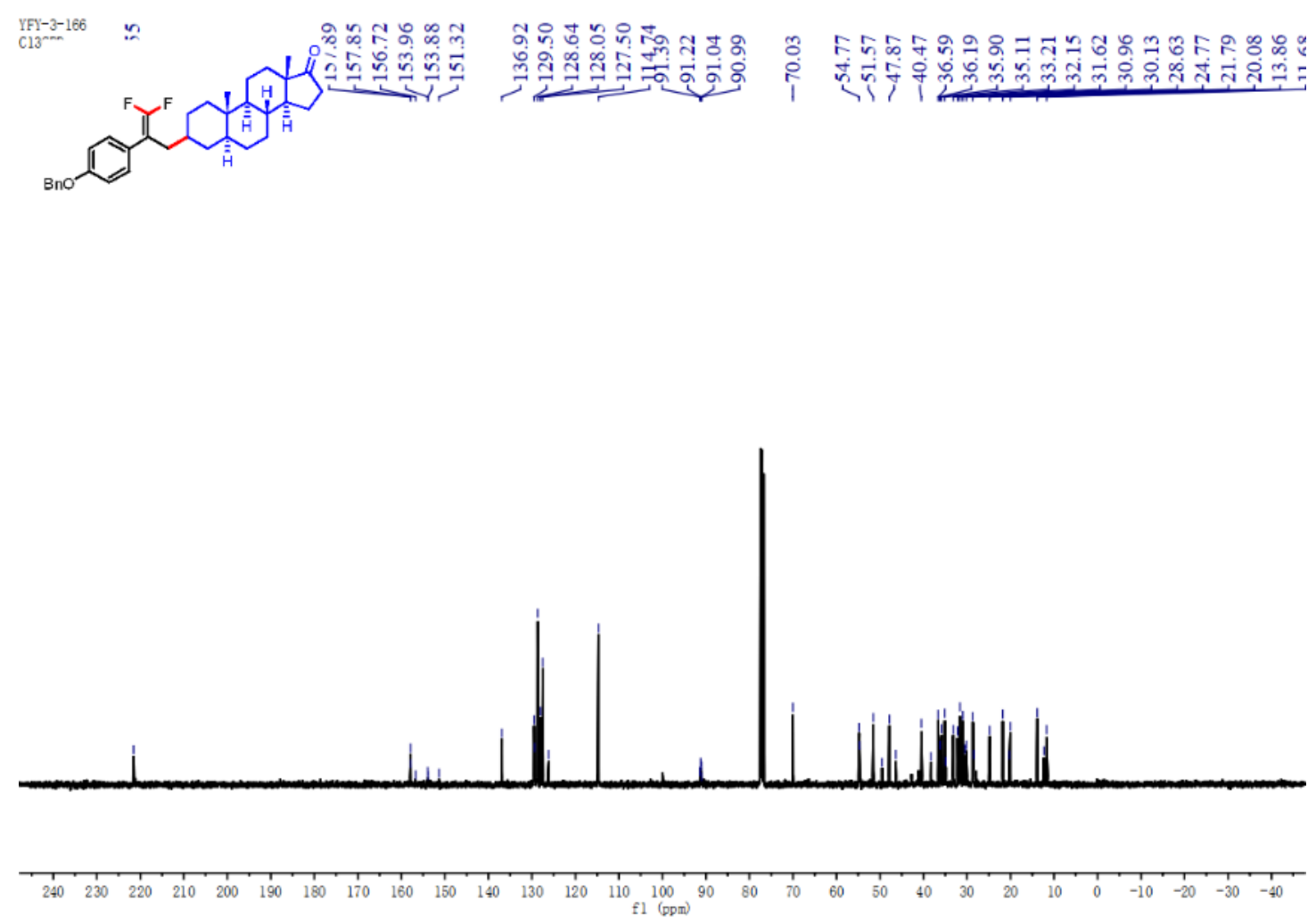

${ }^{19} \mathrm{~F}$ NMR (376 MHz, $\mathrm{CDCl}_{3}$ ) spectrum of compound 24
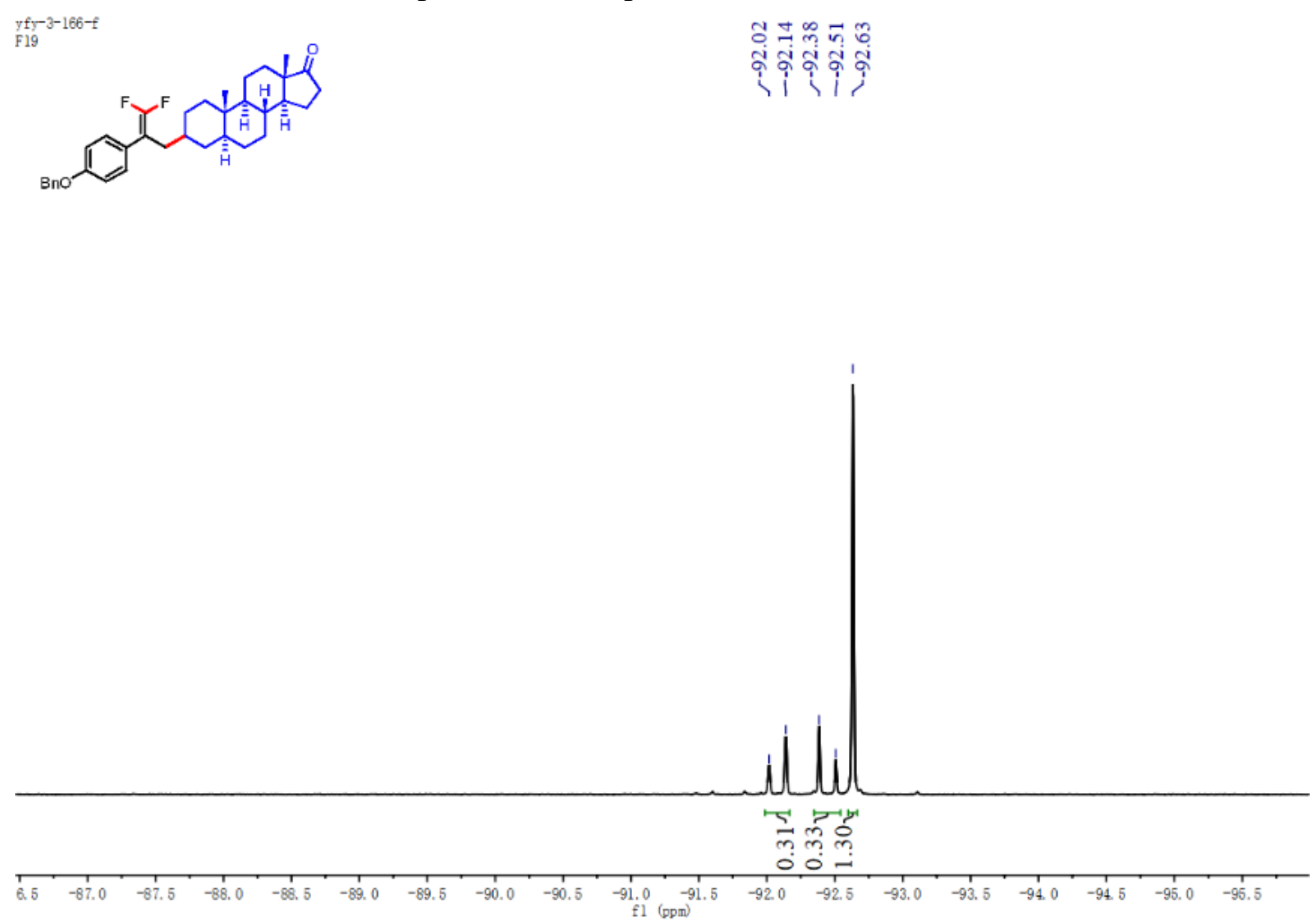

${ }^{1} \mathrm{H}$ NMR (400 MHz, $\mathrm{CDCl}_{3}$ ) spectrum of compound 25 


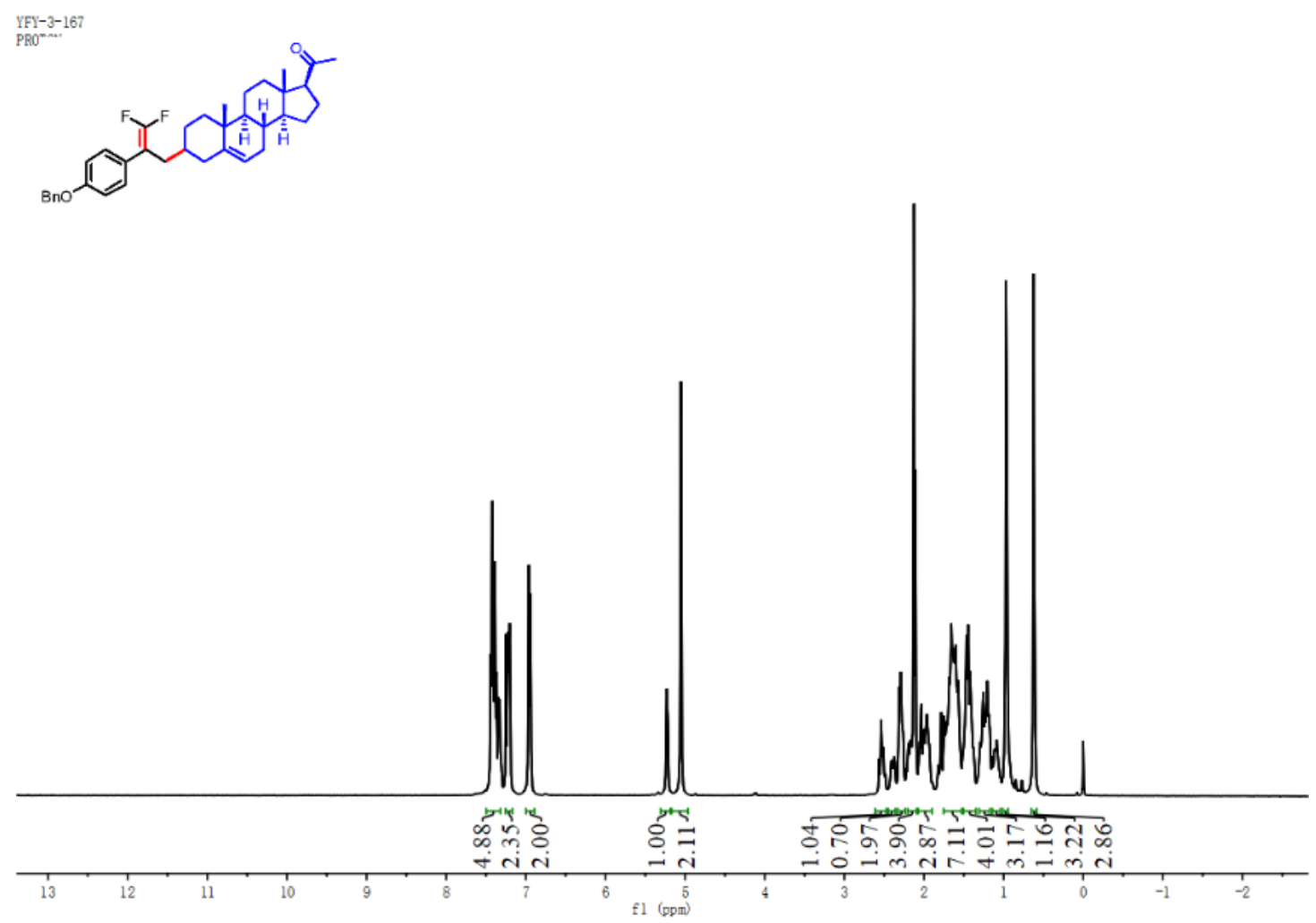

${ }^{13} \mathrm{C}$ NMR (100 MHz, $\mathrm{CDCl}_{3}$ ) spectrum of compound 25
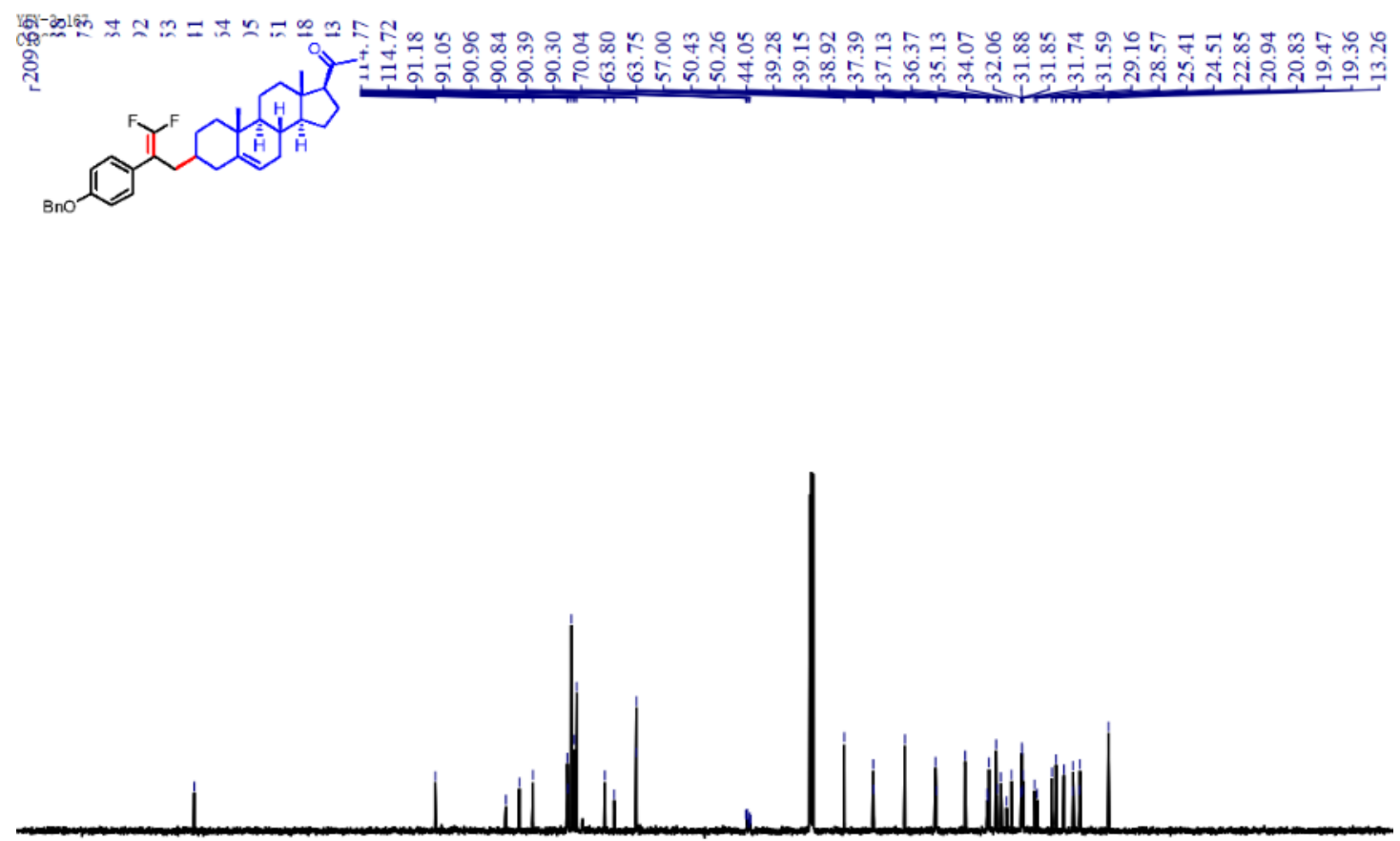

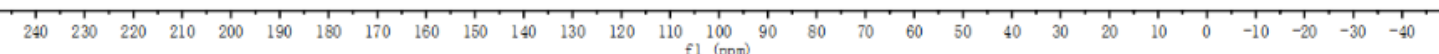

${ }^{19} \mathrm{~F}$ NMR ( $376 \mathrm{MHz}, \mathrm{CDCl}_{3}$ ) spectrum of compound 25 

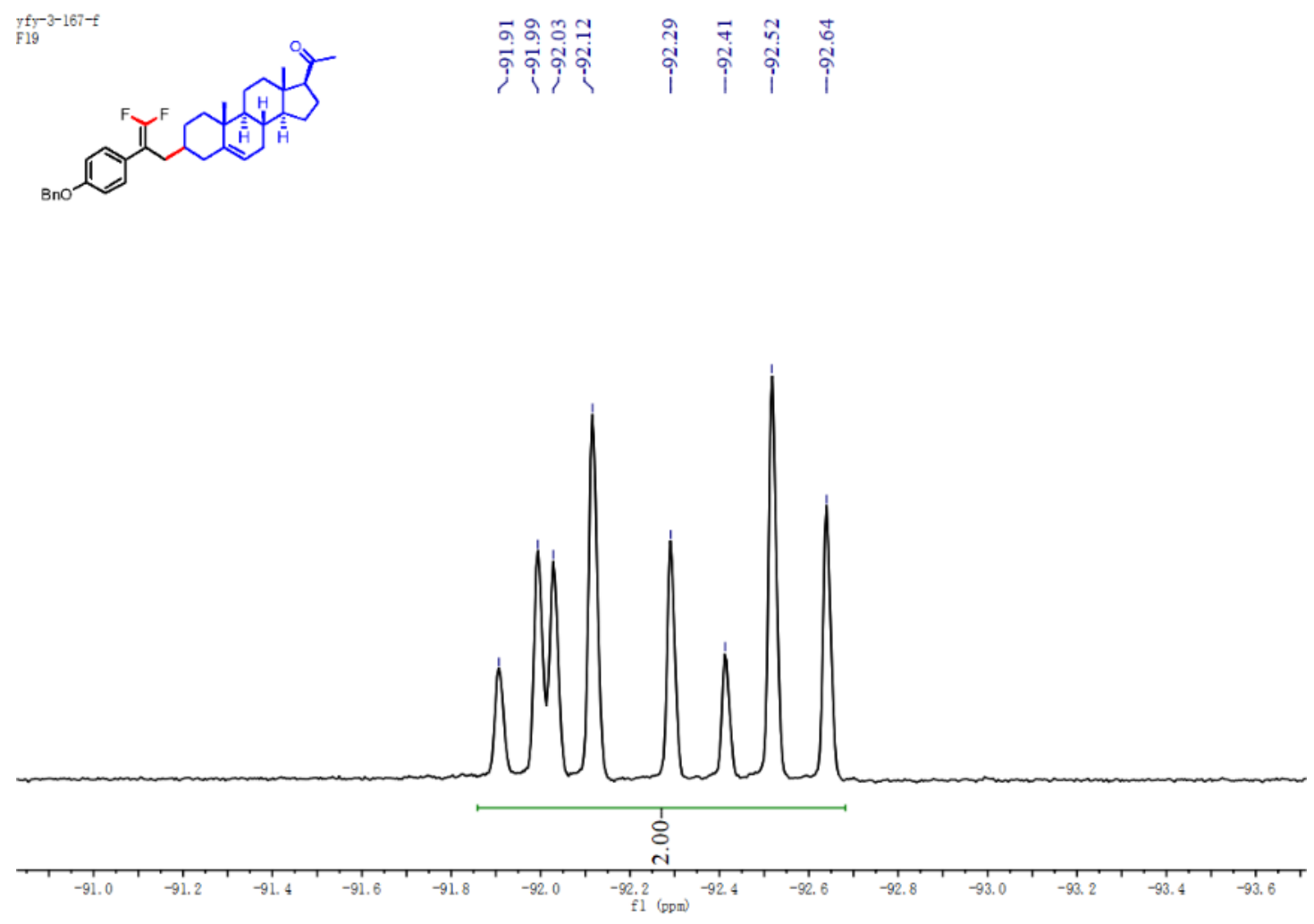

${ }^{1} \mathrm{H}$ NMR (400 MHz, $\mathrm{CDCl}_{3}$ ) spectrum of compound 26
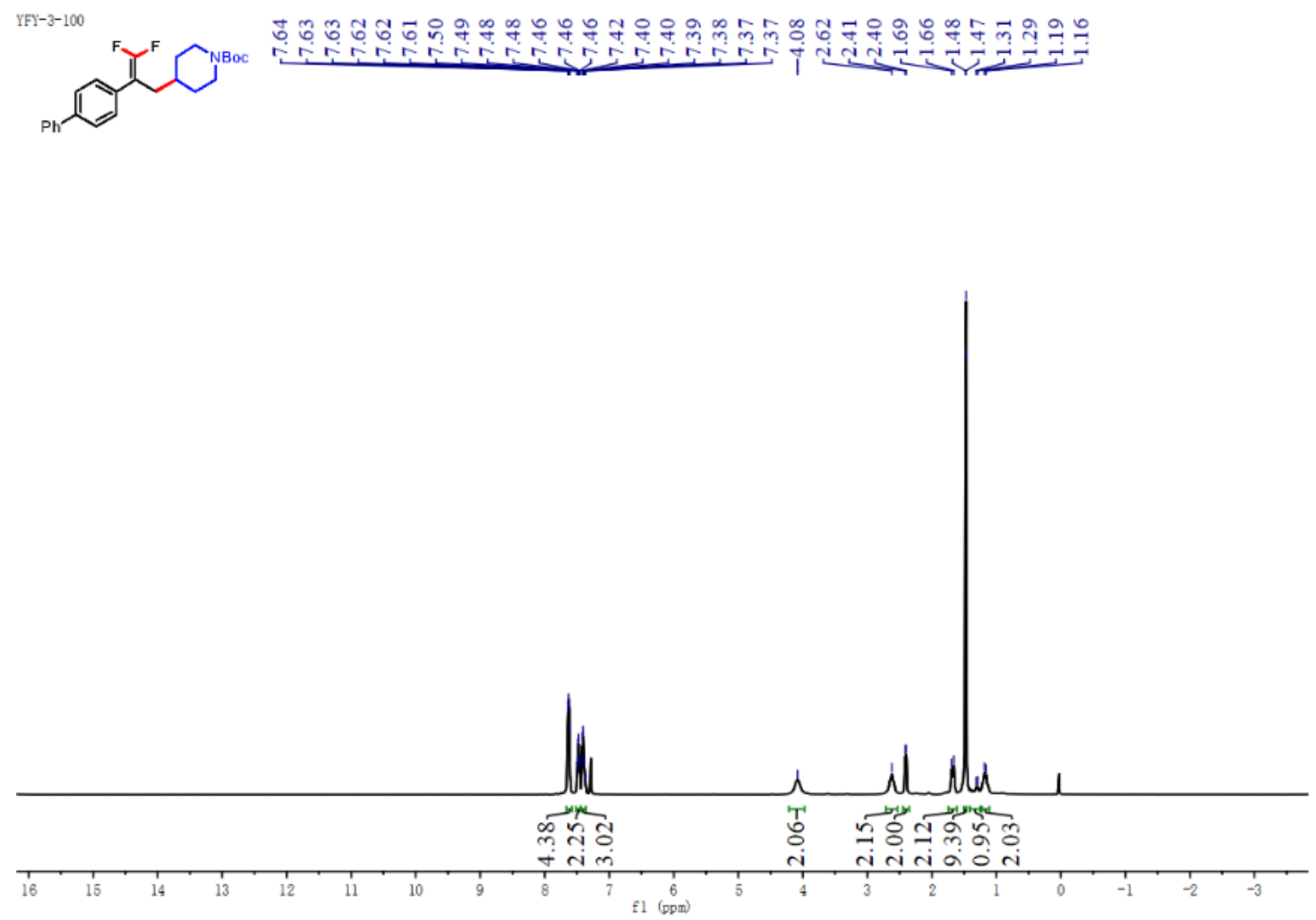

${ }^{13} \mathrm{C}$ NMR (100 MHz, $\mathrm{CDCl}_{3}$ ) spectrum of compound 26 

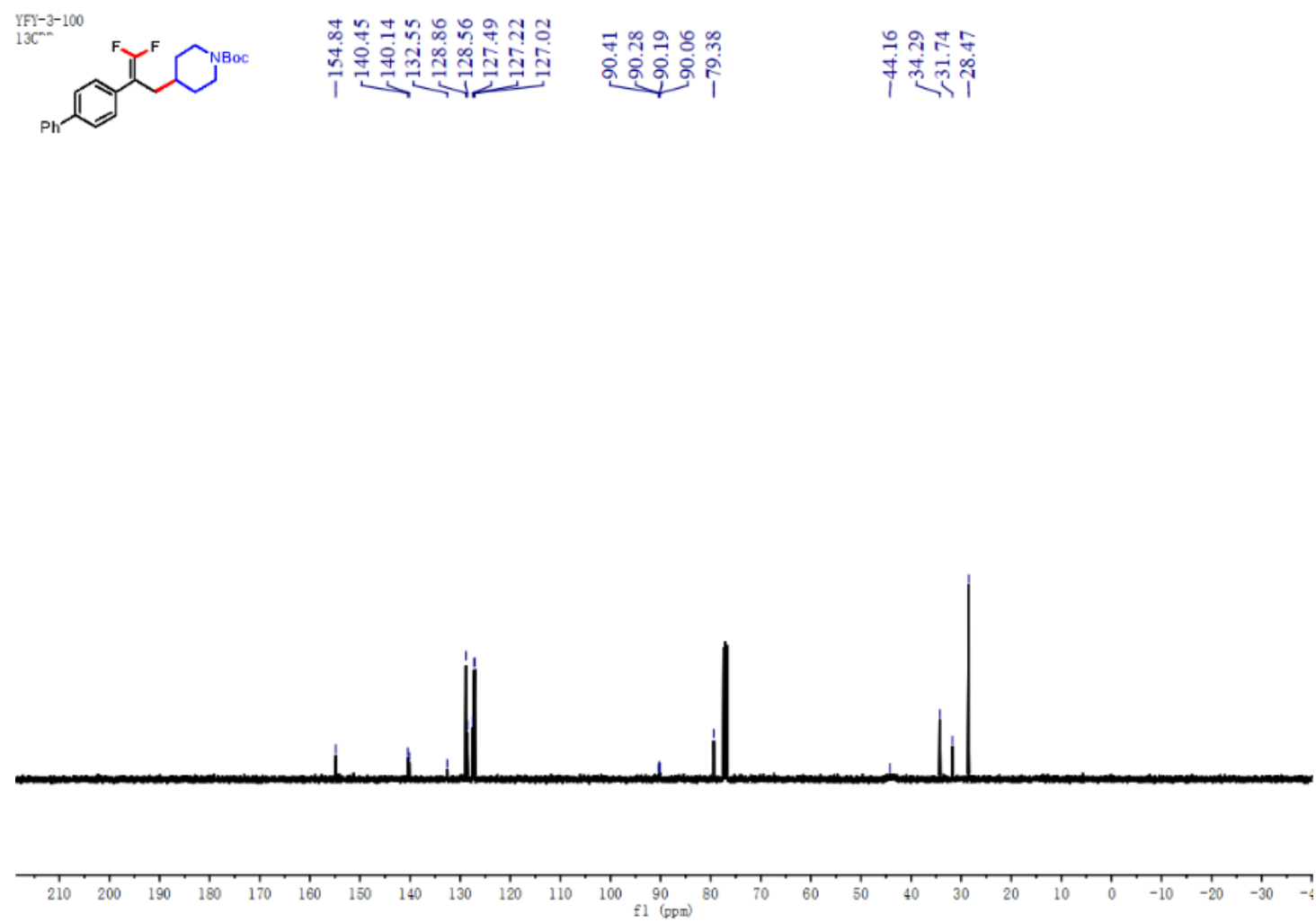

${ }^{19} \mathrm{~F} \mathrm{NMR}\left(376 \mathrm{MHz}, \mathrm{CDCl}_{3}\right.$ ) spectrum of compound 26
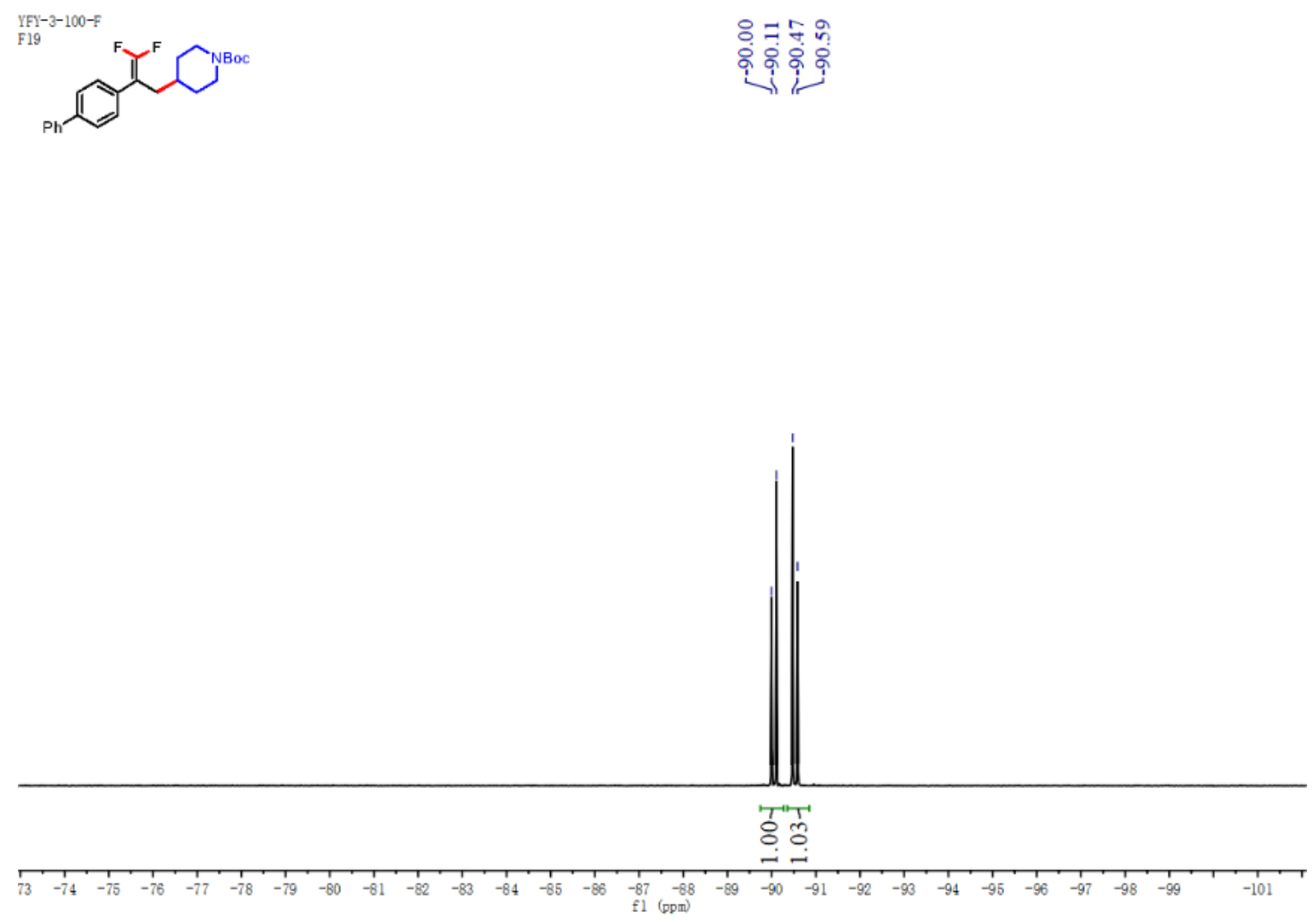

${ }^{1} \mathrm{H}$ NMR (400 MHz, $\mathrm{CDCl}_{3}$ ) spectrum of compound 27 

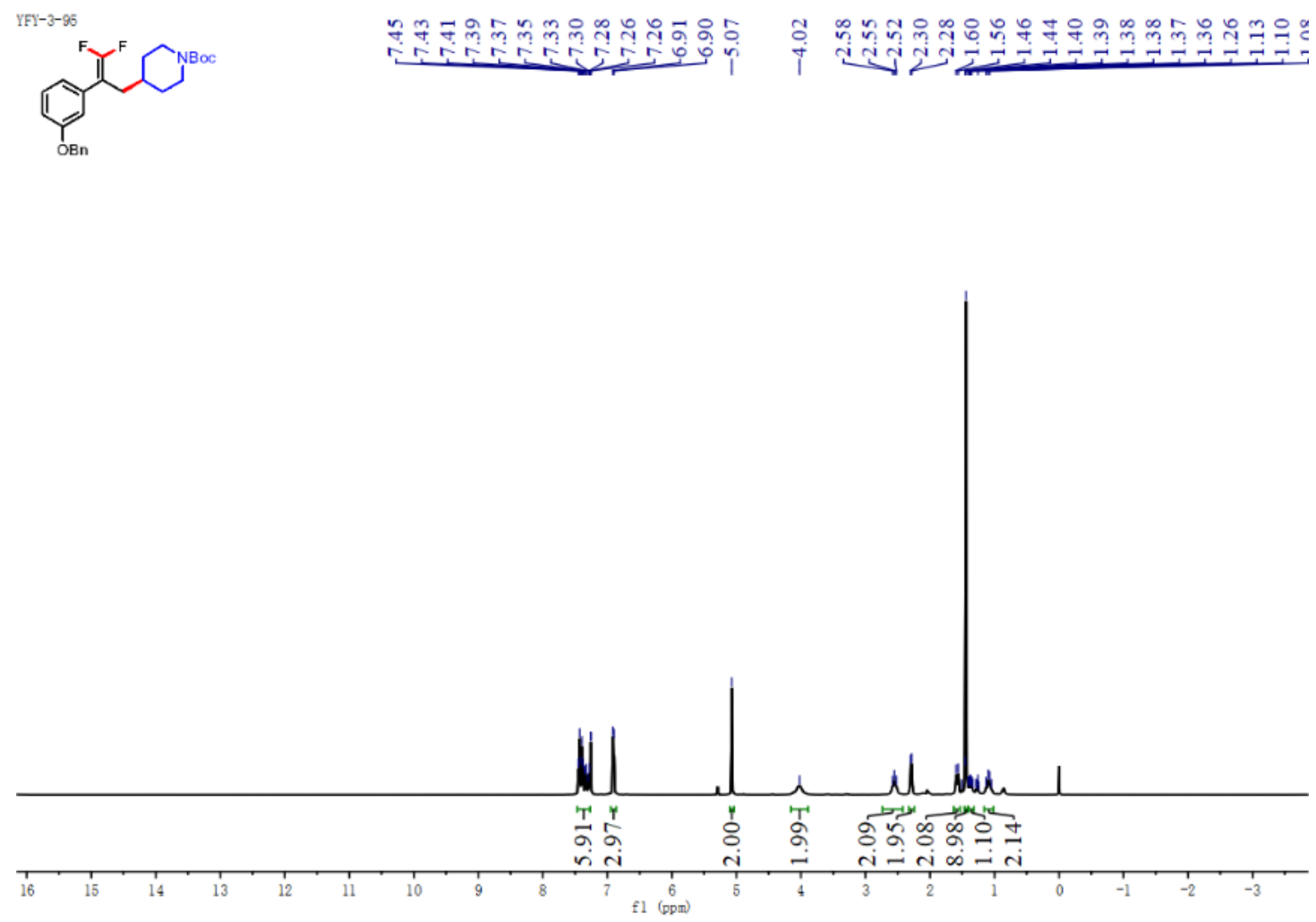

${ }^{13} \mathrm{C}$ NMR $\left(100 \mathrm{MHz}, \mathrm{CDCl}_{3}\right)$ spectrum of compound 27
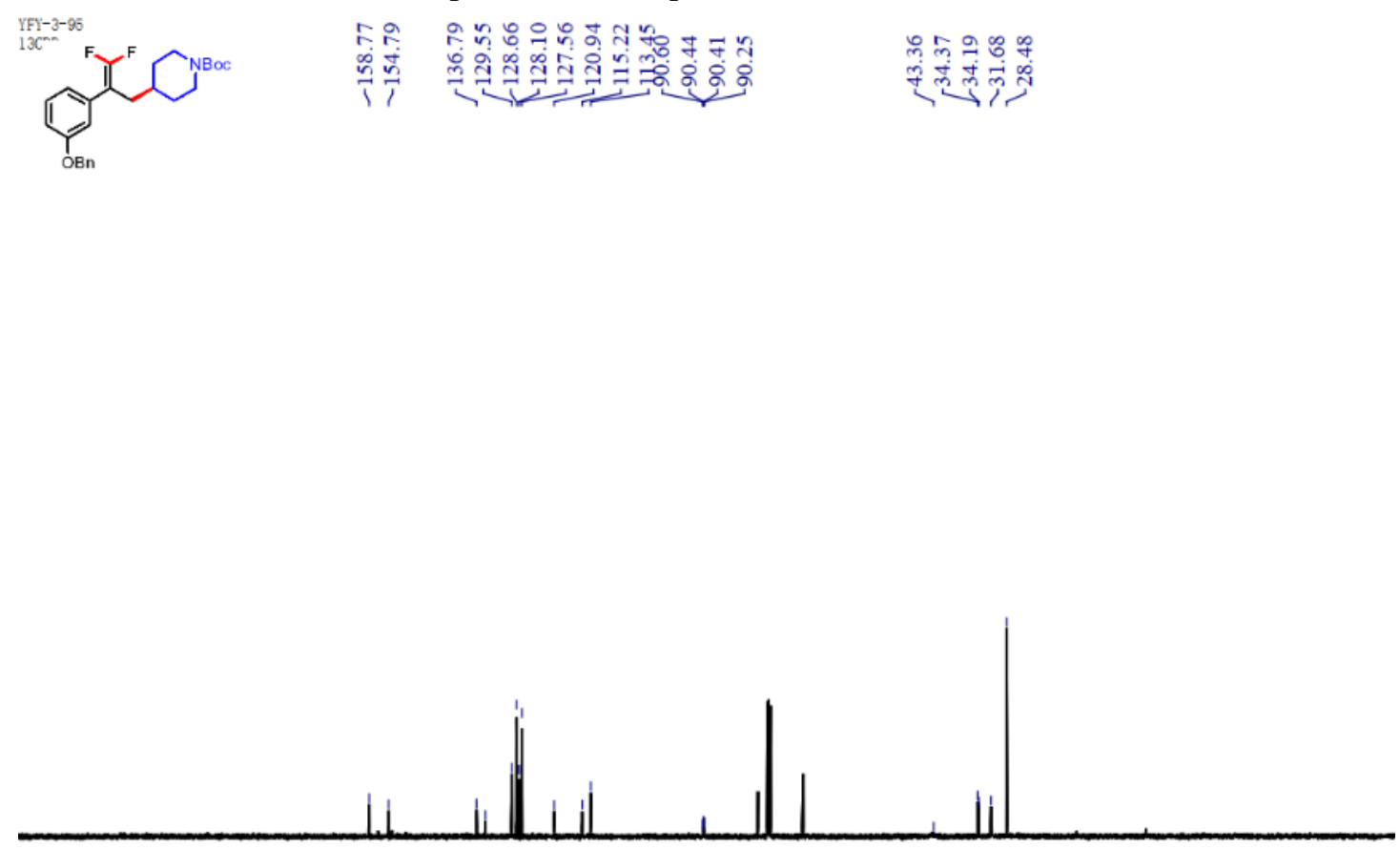

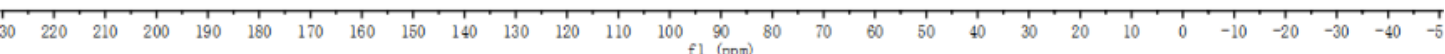

${ }^{19} \mathrm{~F}$ NMR ( $376 \mathrm{MHz}, \mathrm{CDCl}_{3}$ ) spectrum of compound 27 

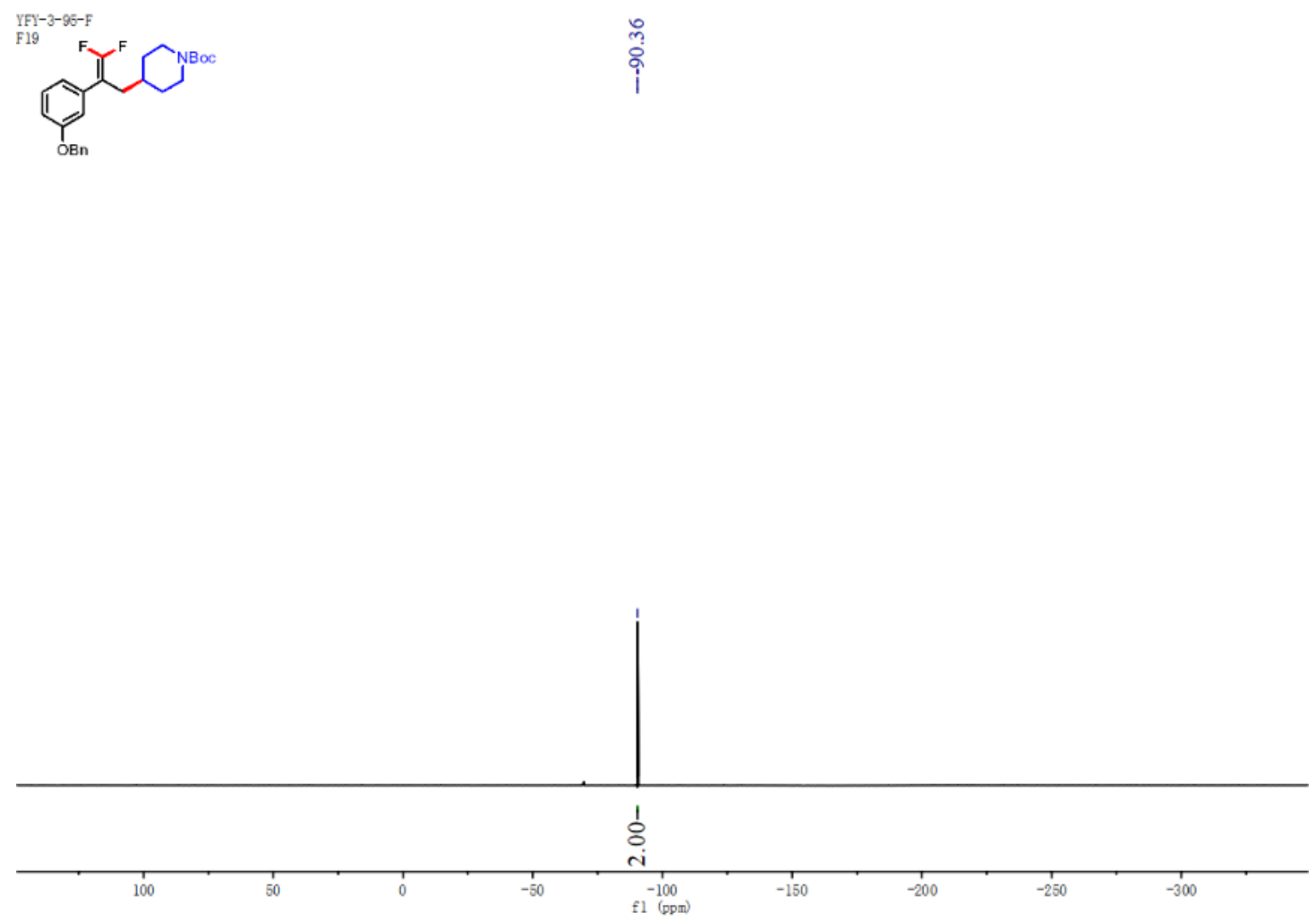

${ }^{1} \mathrm{H}$ NMR (400 MHz, $\mathrm{CDCl}_{3}$ ) spectrum of compound 28
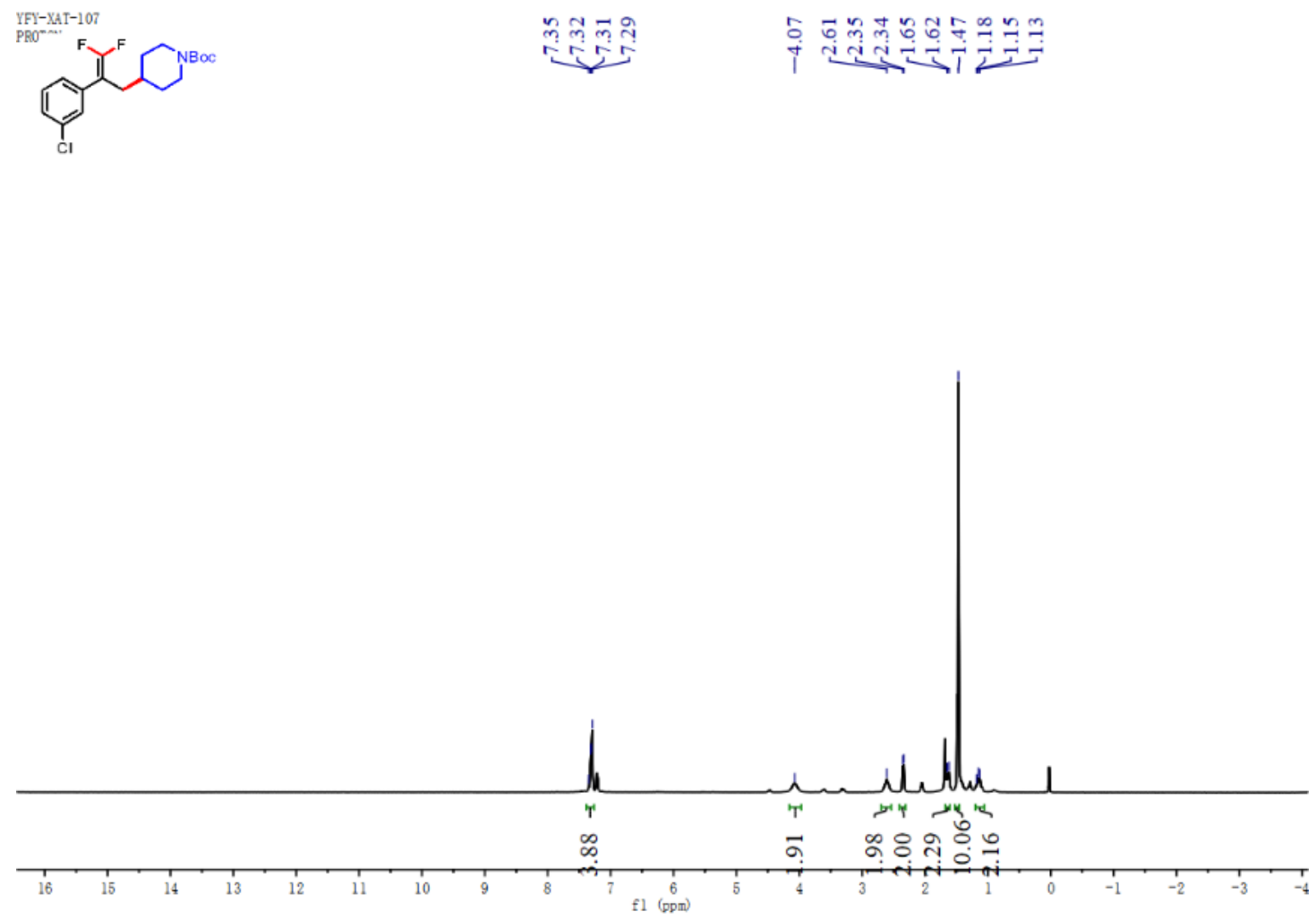

${ }^{13} \mathrm{C}$ NMR (100 MHz, $\mathrm{CDCl}_{3}$ ) spectrum of compound 28 

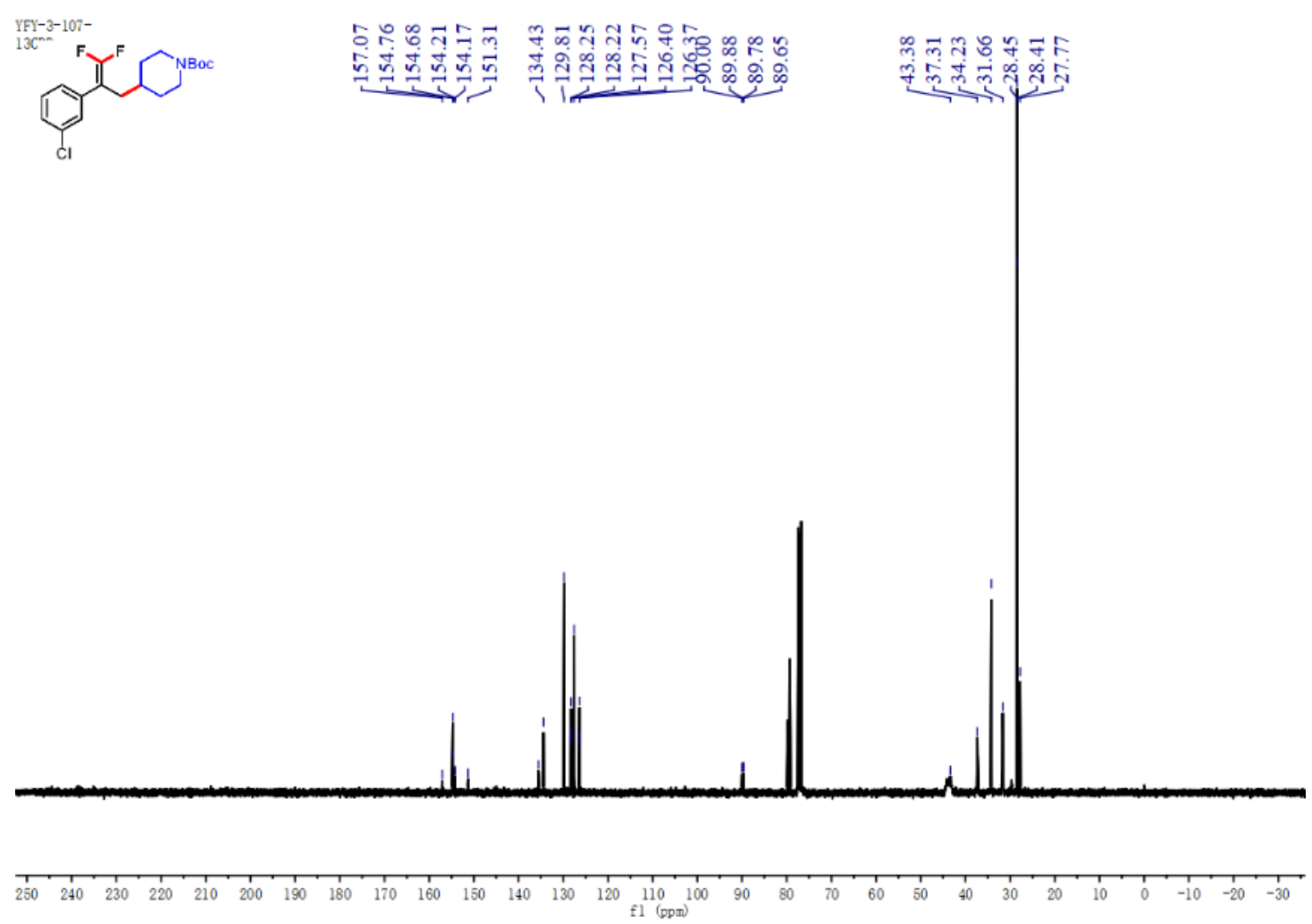

${ }^{19} \mathrm{~F}$ NMR (376 MHz, $\mathrm{CDCl}_{3}$ ) spectrum of compound 28
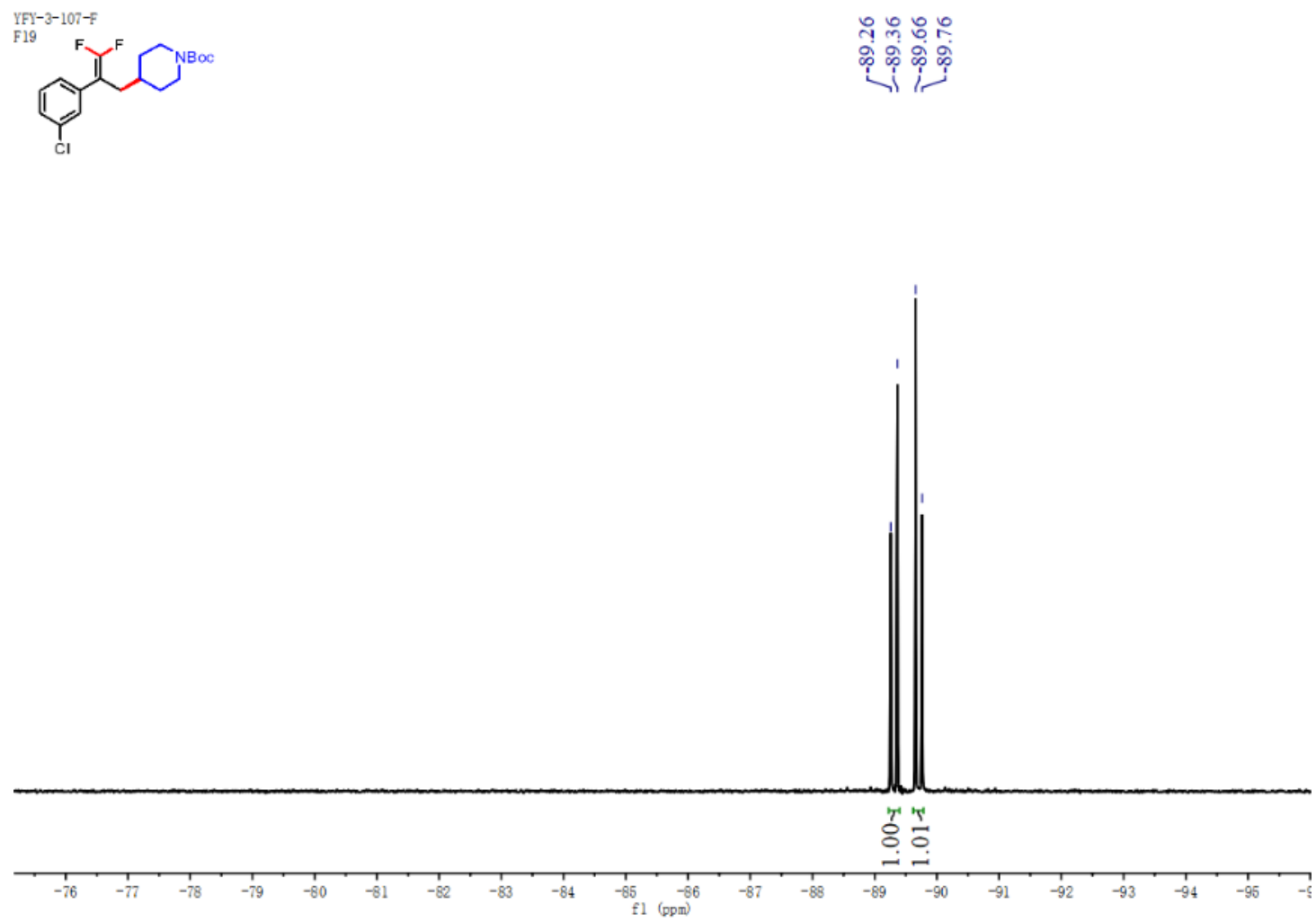

${ }^{1} \mathrm{H}$ NMR (400 MHz, $\mathrm{CDCl}_{3}$ ) spectrum of compound 29 

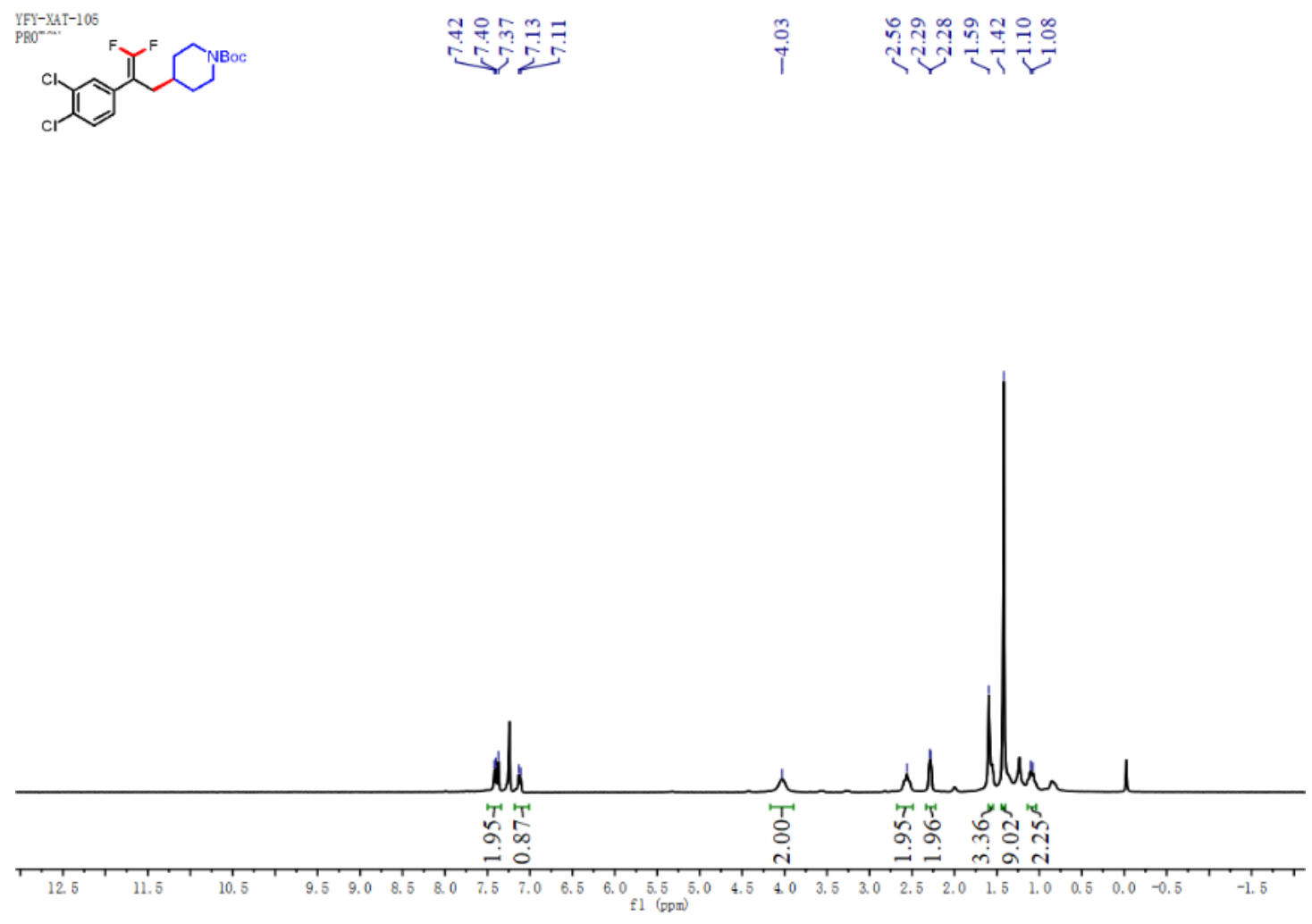

${ }^{13} \mathrm{C}$ NMR (100 MHz, $\mathrm{CDCl}_{3}$ ) spectrum of compound 29
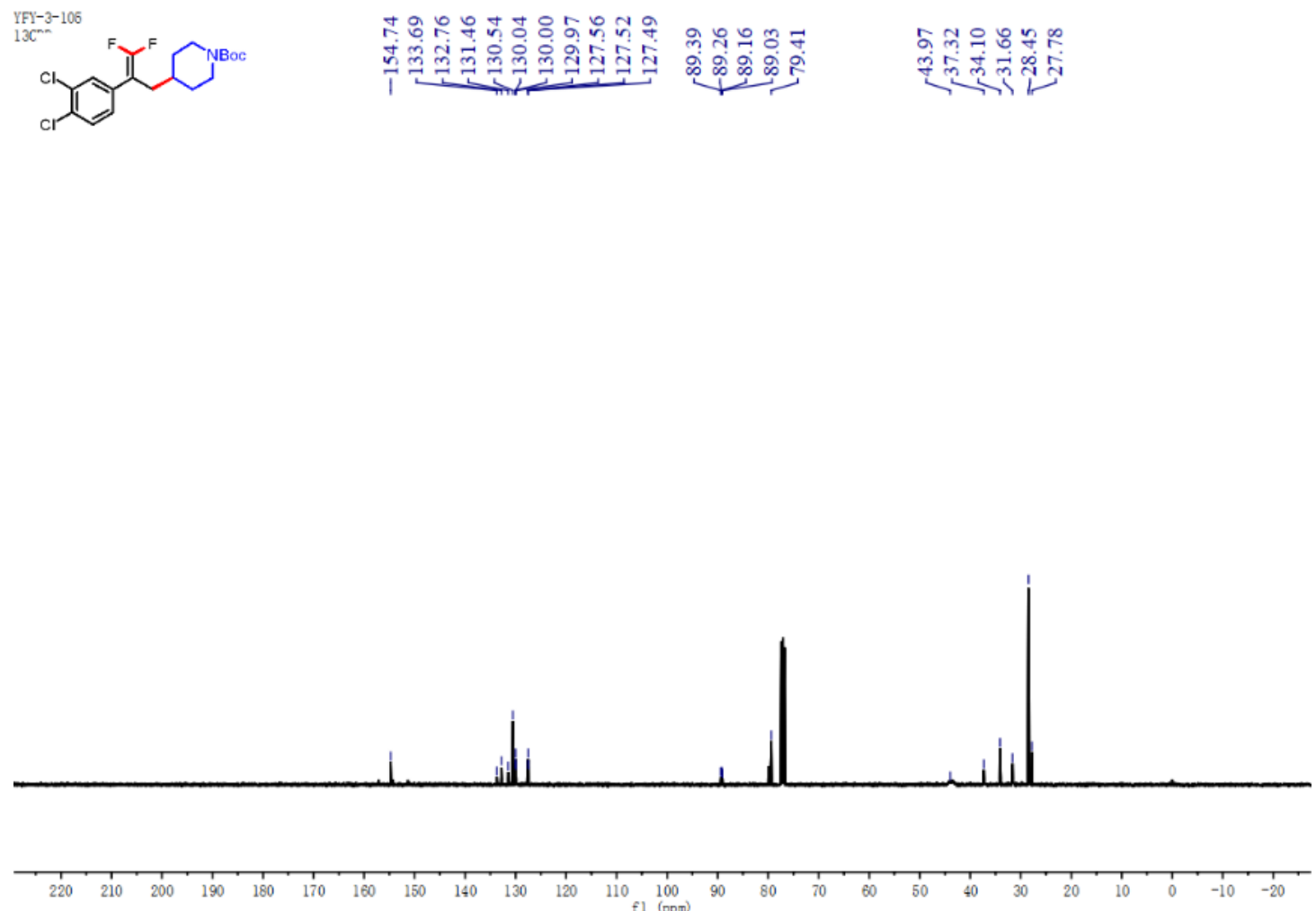

${ }^{19}$ F NMR (376 MHz, $\mathrm{CDCl}_{3}$ ) spectrum of compound 29 

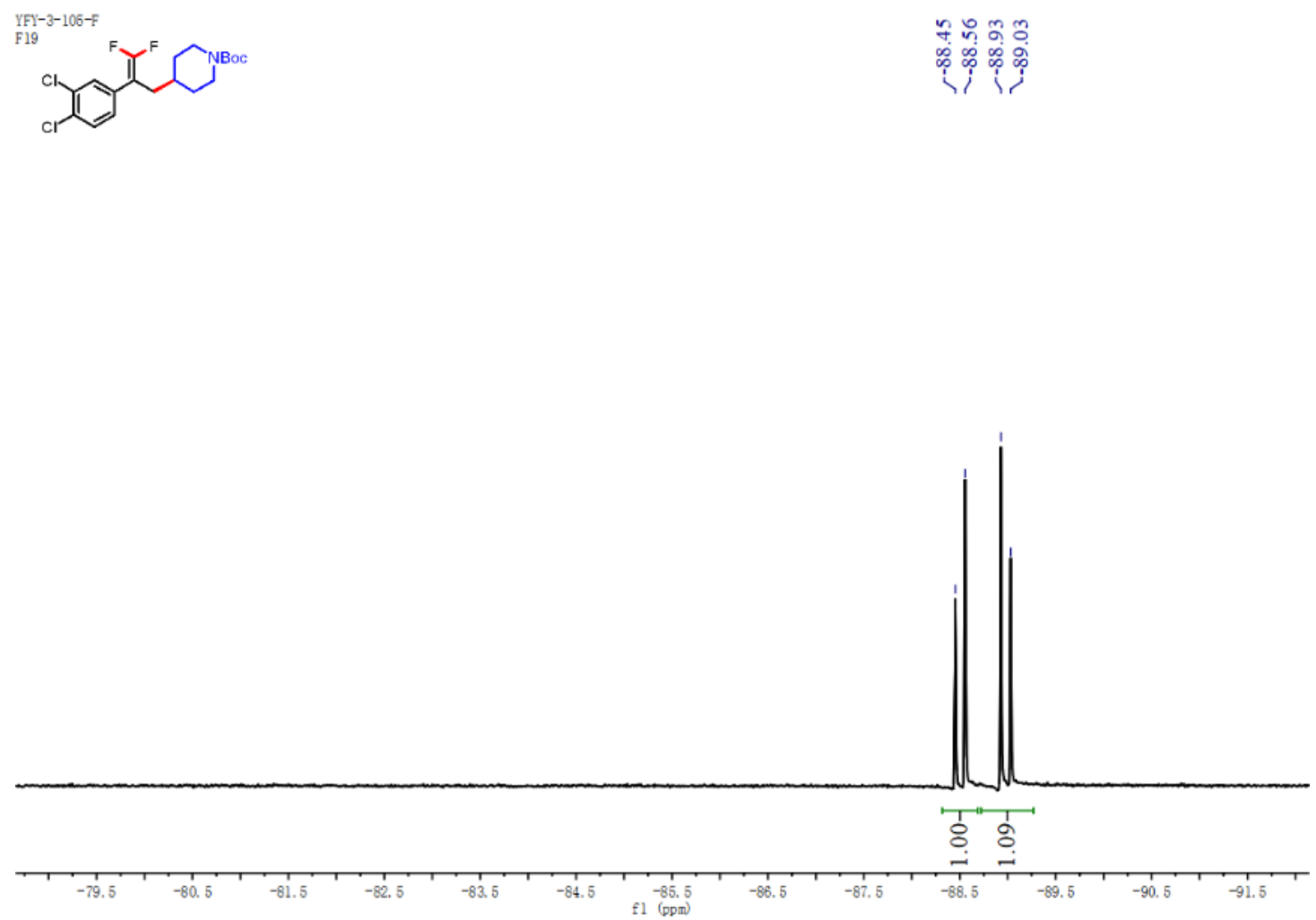

${ }^{1} \mathrm{H}$ NMR (400 MHz, $\mathrm{CDCl}_{3}$ ) spectrum of compound $\mathbf{3 0}$
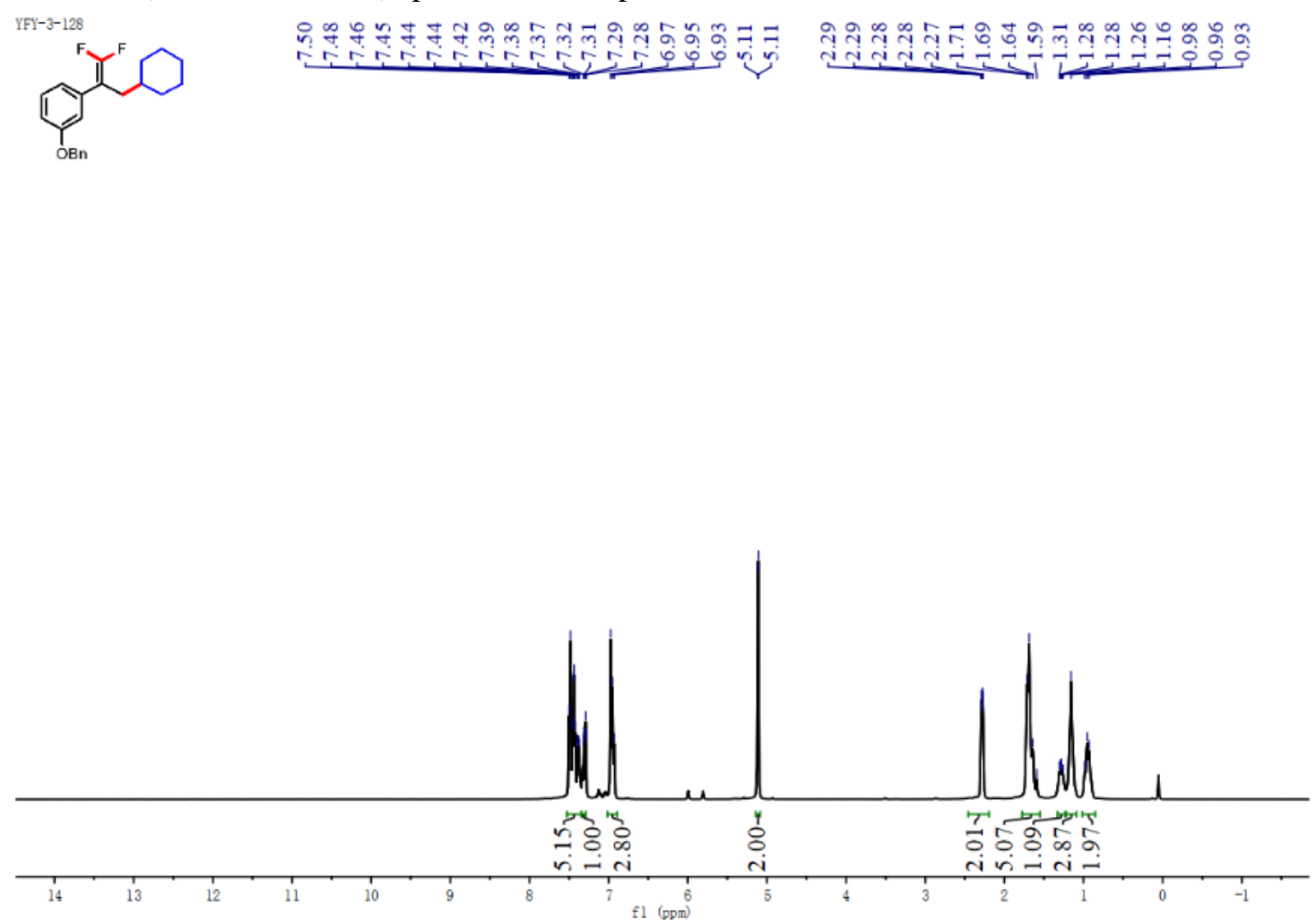

${ }^{13} \mathrm{C}$ NMR (100 MHz, $\mathrm{CDCl}_{3}$ ) spectrum of compound 30 

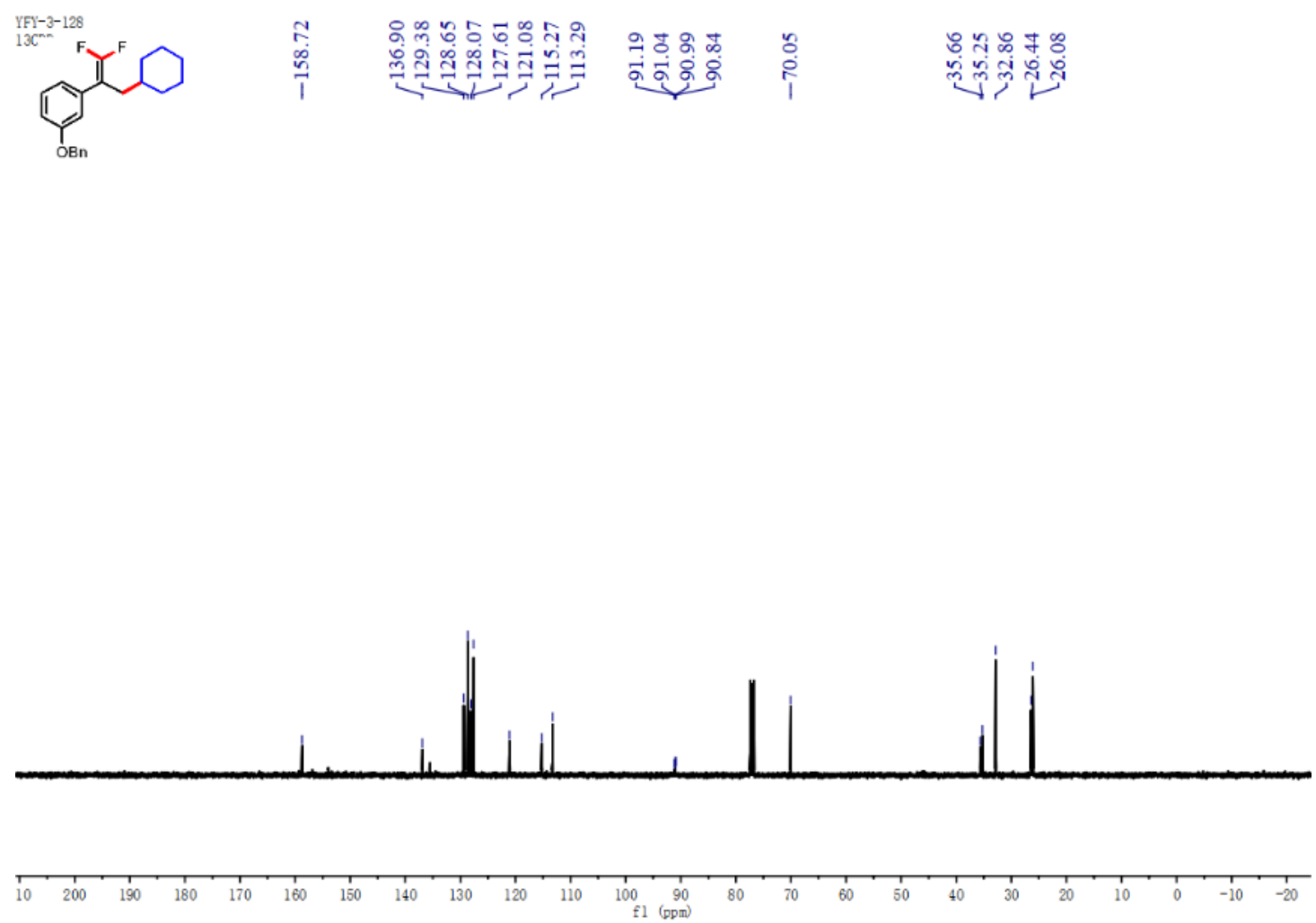

${ }^{19} \mathrm{~F}$ NMR (376 MHz, $\mathrm{CDCl}_{3}$ ) spectrum of compound $\mathbf{3 0}$

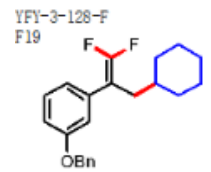

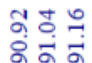

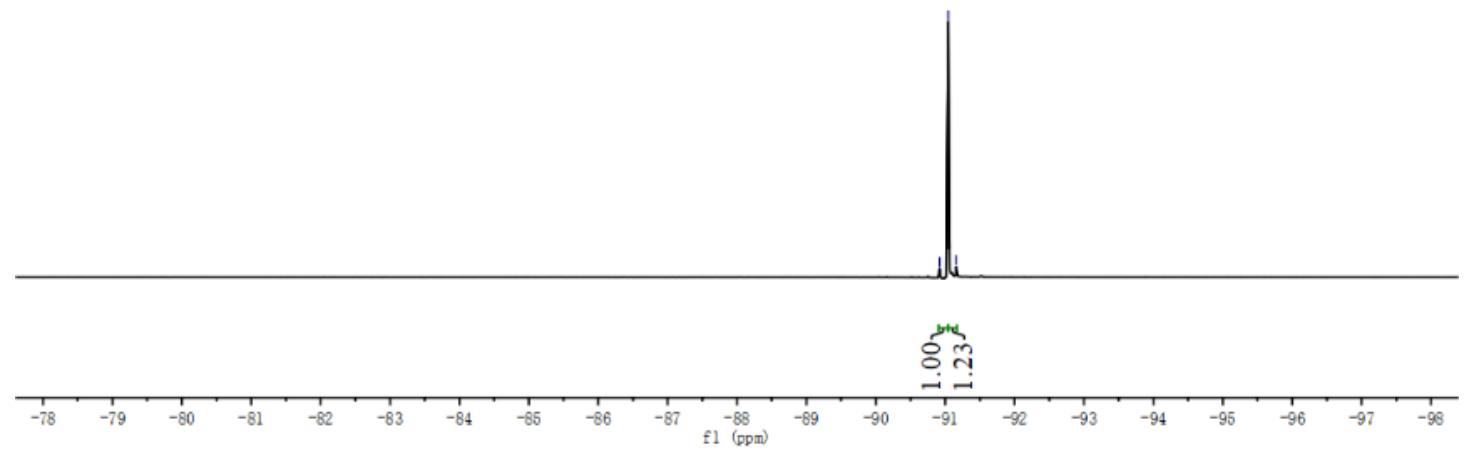

${ }^{1} \mathrm{H}$ NMR (400 MHz, $\mathrm{CDCl}_{3}$ ) spectrum of compound 31 

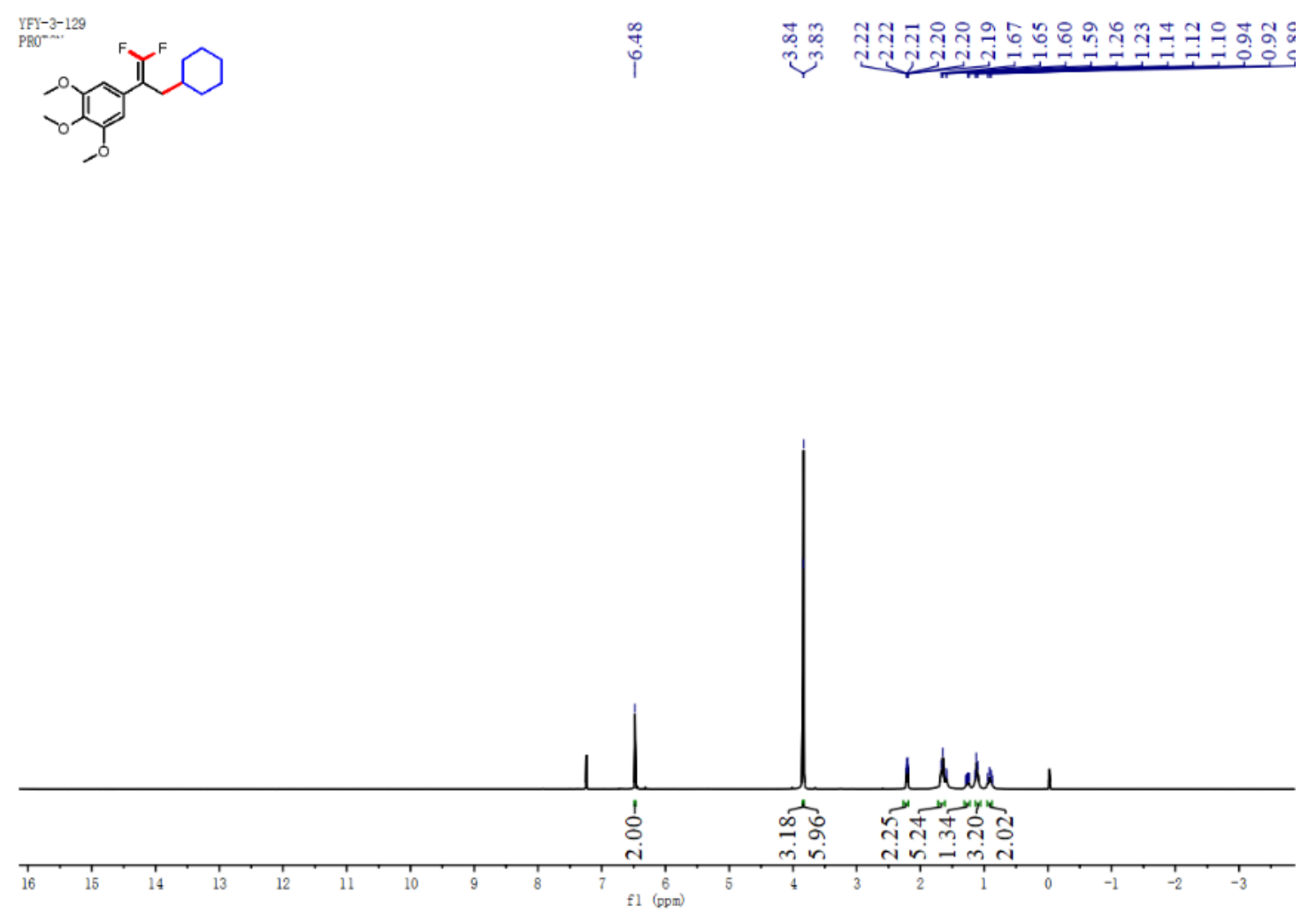

${ }^{13} \mathrm{C}$ NMR (100 MHz, $\mathrm{CDCl}_{3}$ ) spectrum of compound $\mathbf{3 1}$
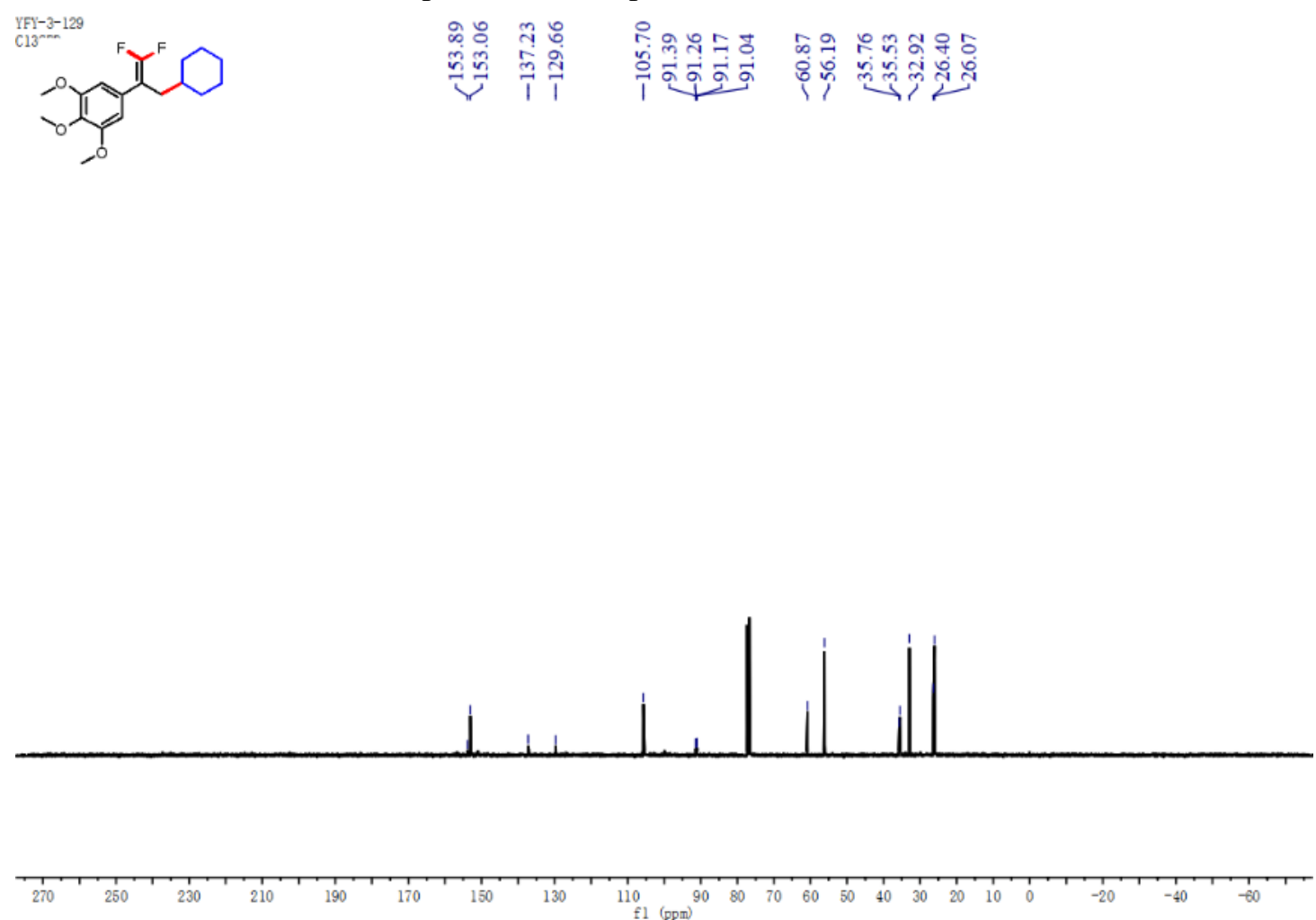

${ }^{19} \mathrm{~F}$ NMR (376 MHz, $\mathrm{CDCl}_{3}$ ) spectrum of compound $\mathbf{3 1}$ 


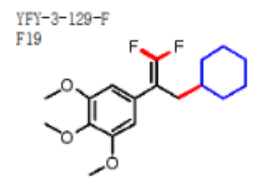

원

휘항

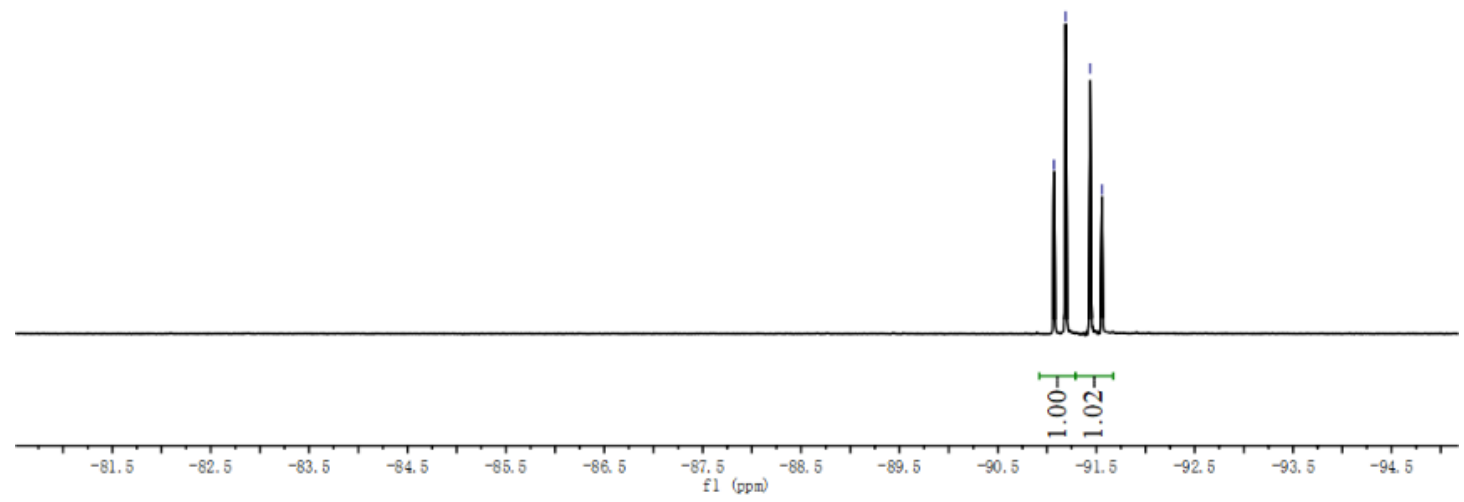

${ }^{1} \mathrm{H}$ NMR (400 MHz, $\mathrm{CDCl}_{3}$ ) spectrum of compound 32
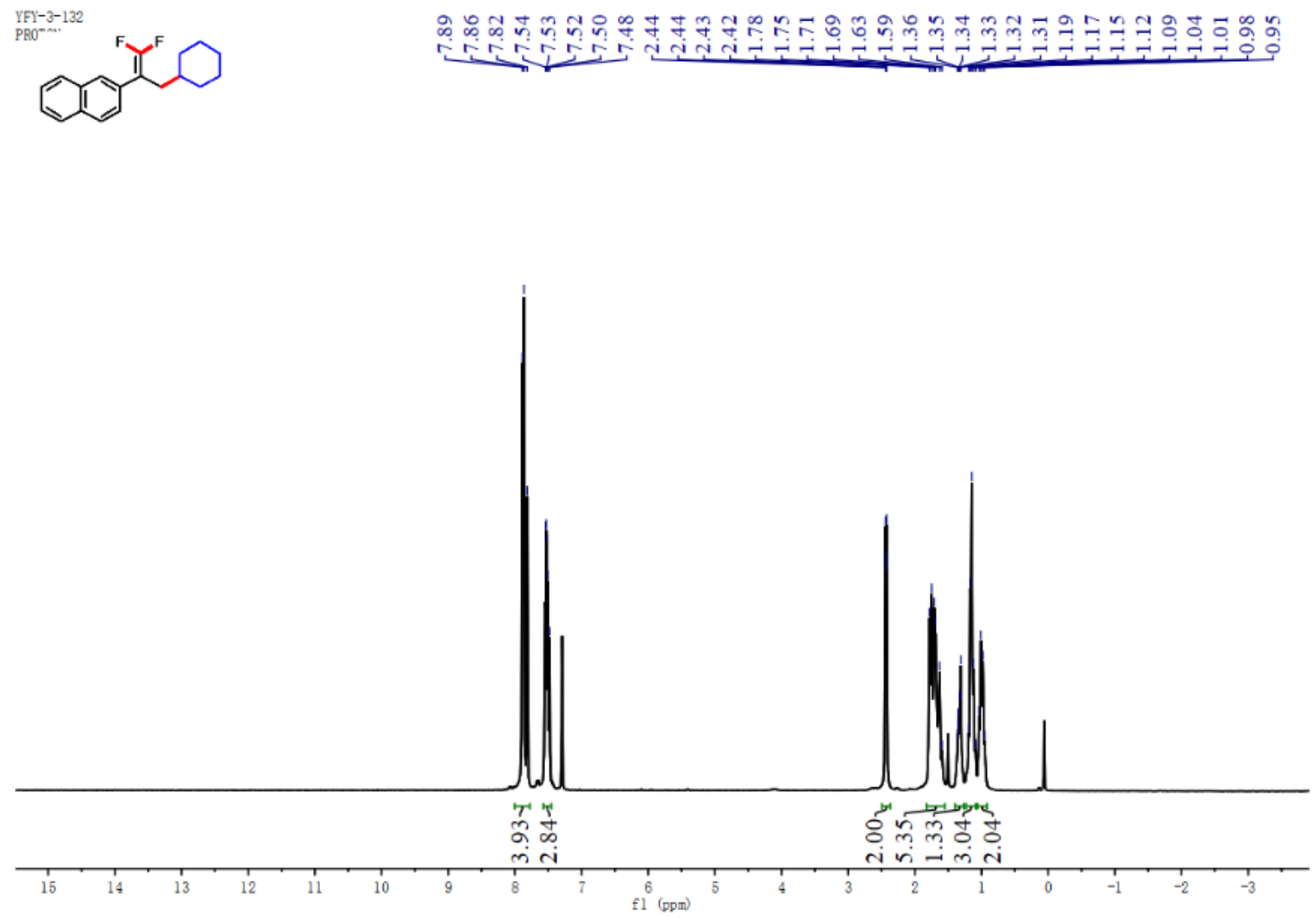

${ }^{13} \mathrm{C}$ NMR (100 MHz, $\mathrm{CDCl}_{3}$ ) spectrum of compound 32 

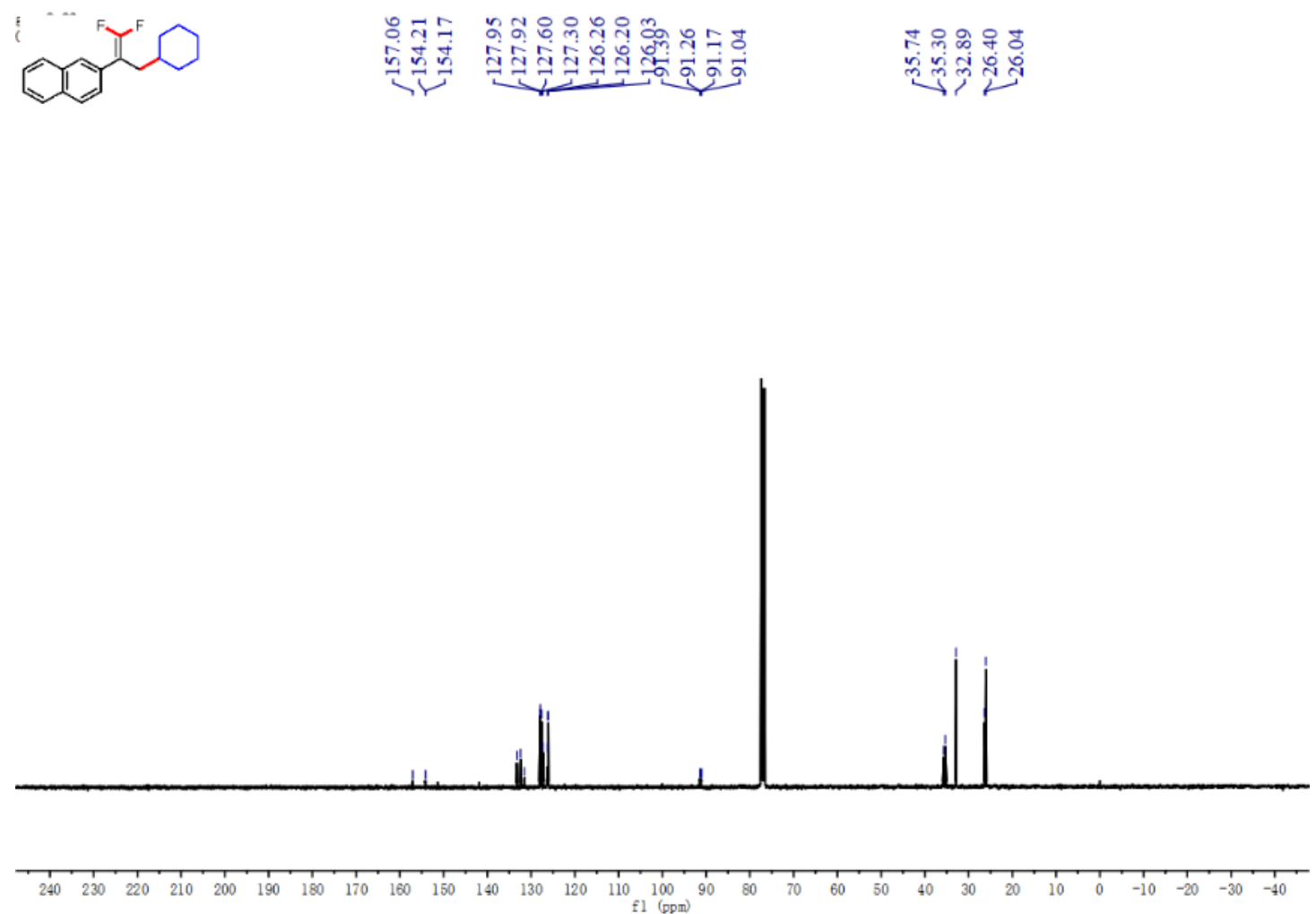

${ }^{19} \mathrm{~F}$ NMR $\left(376 \mathrm{MHz}, \mathrm{CDCl}_{3}\right.$ ) spectrum of compound 32

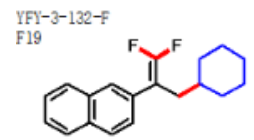

$\infty \vec{a} \overrightarrow{0}$

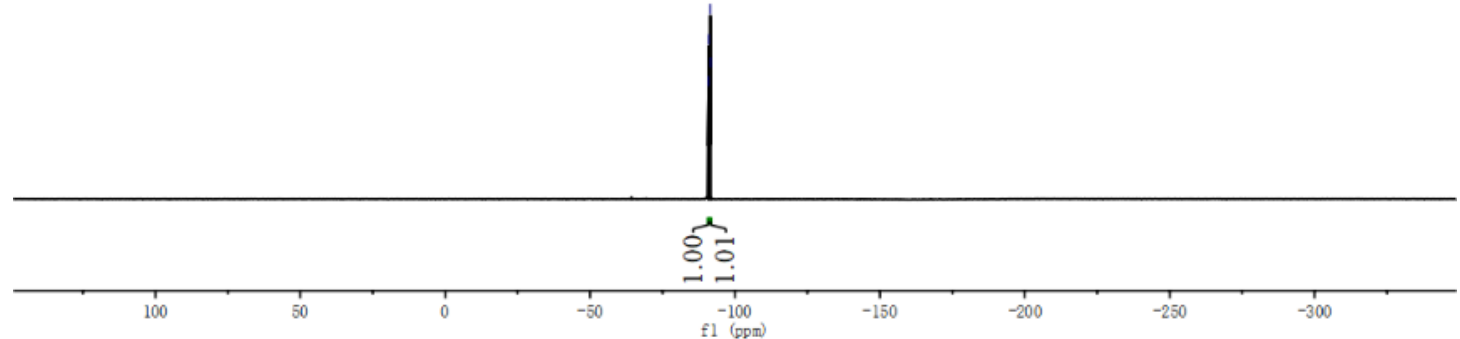

${ }^{1} \mathrm{H}$ NMR $\left(400 \mathrm{MHz}, \mathrm{CDCl}_{3}\right.$ ) spectrum of compound $\mathbf{3 3}$ 

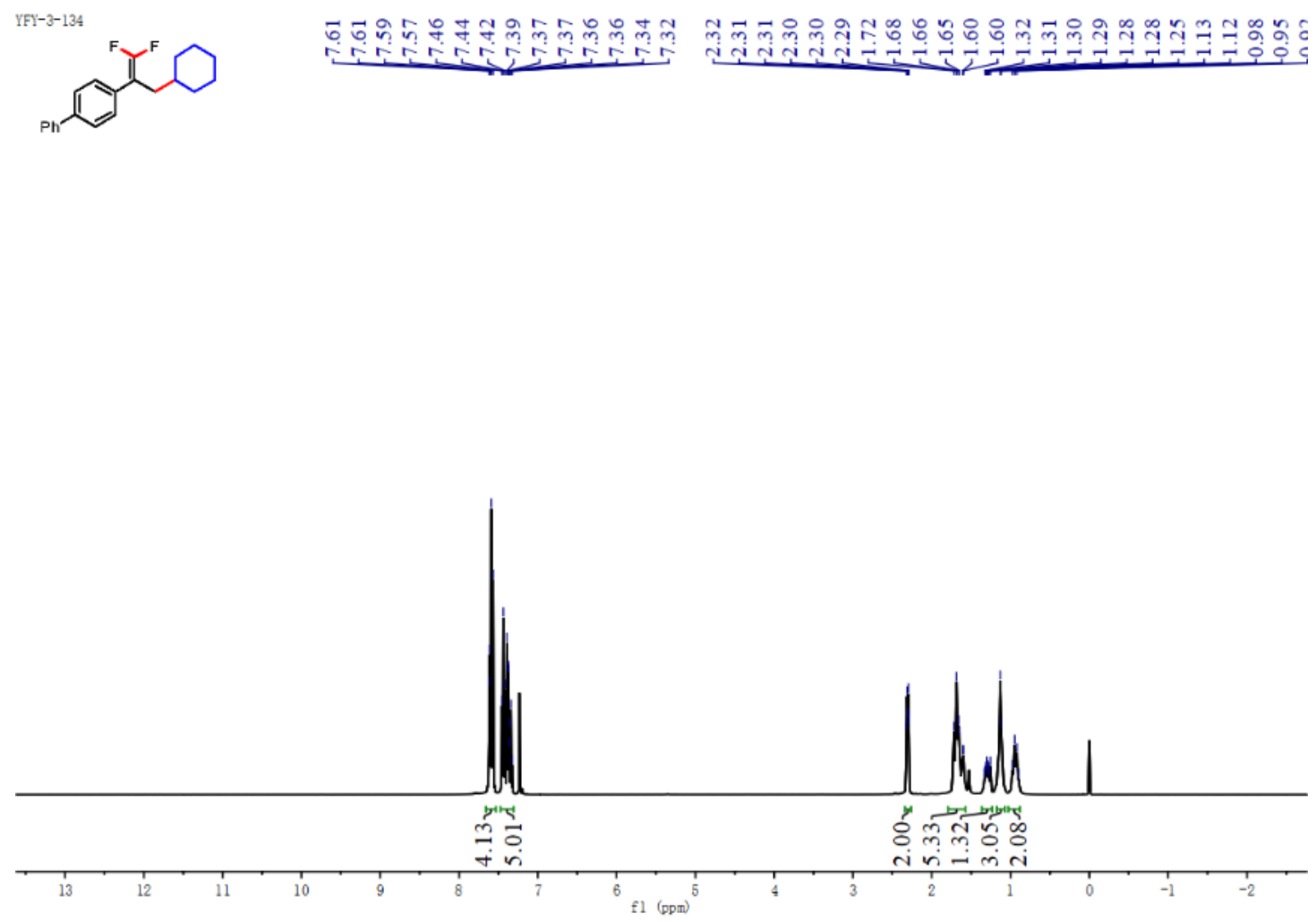

${ }^{13} \mathrm{C}$ NMR (100 MHz, $\mathrm{CDCl}_{3}$ ) spectrum of compound $\mathbf{3 3}$
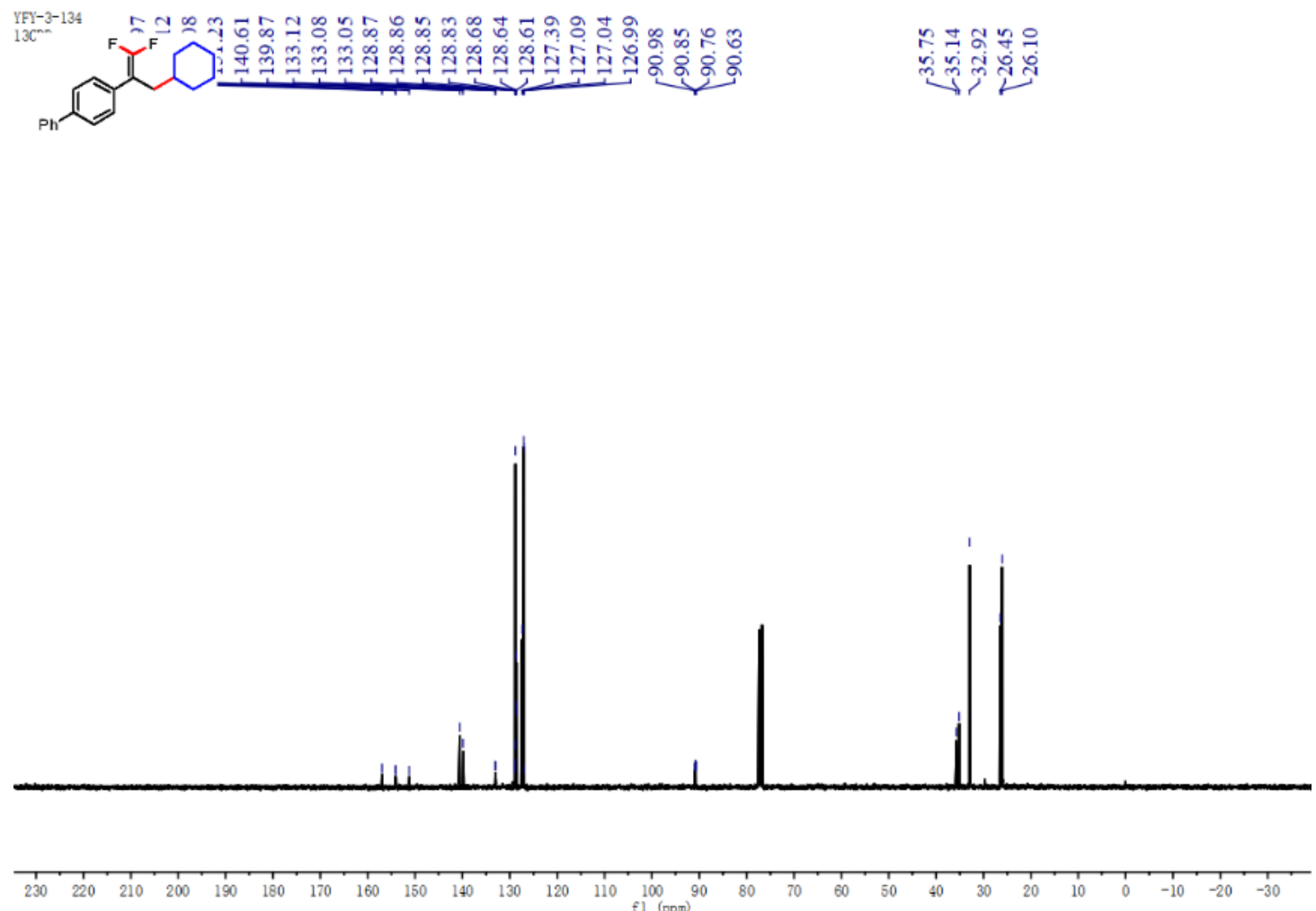

${ }^{19} \mathrm{~F}$ NMR $\left(376 \mathrm{MHz}, \mathrm{CDCl}_{3}\right.$ ) spectrum of compound 33 


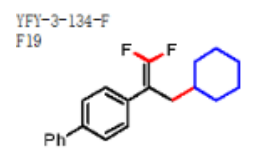

두누도

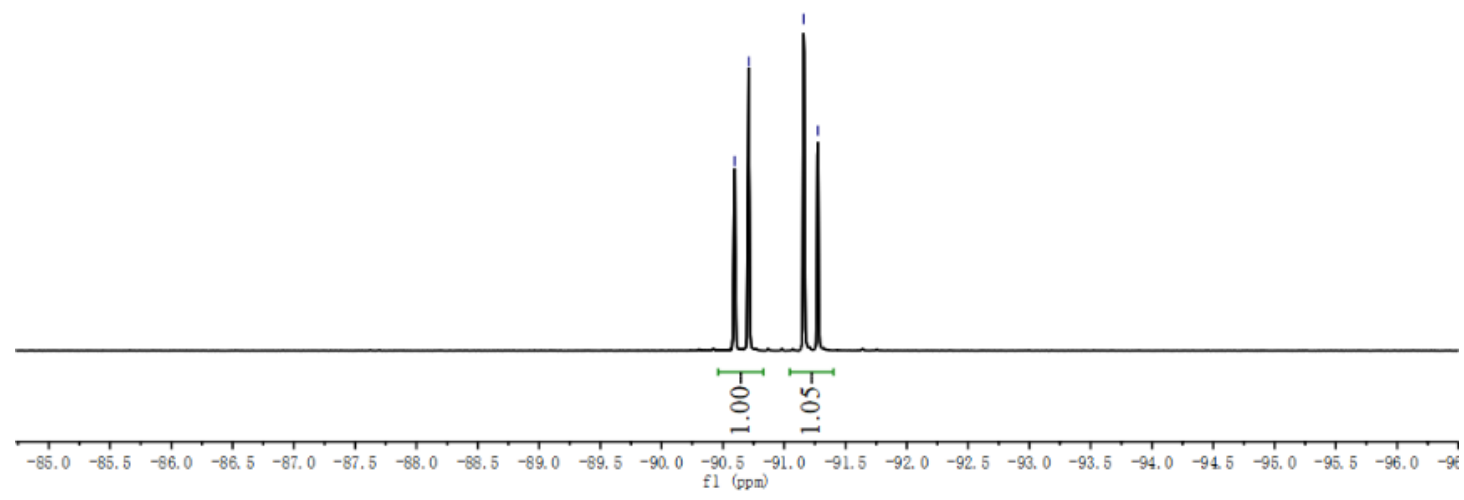

${ }^{1} \mathrm{H}$ NMR (400 MHz, $\mathrm{CDCl}_{3}$ ) spectrum of compound $\mathbf{3 4}$
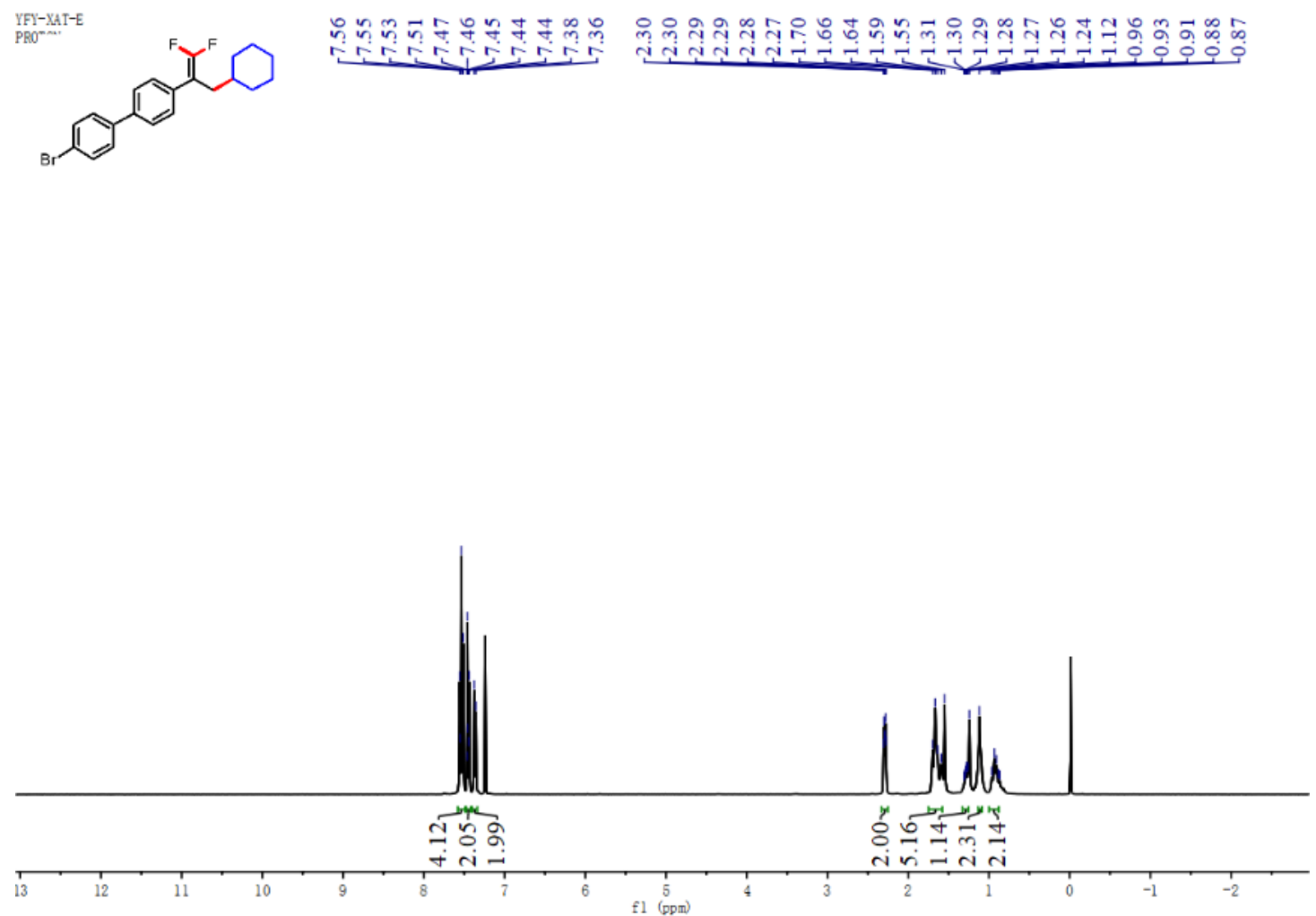

${ }^{13} \mathrm{C}$ NMR (100 MHz, $\mathrm{CDCl}_{3}$ ) spectrum of compound 34 

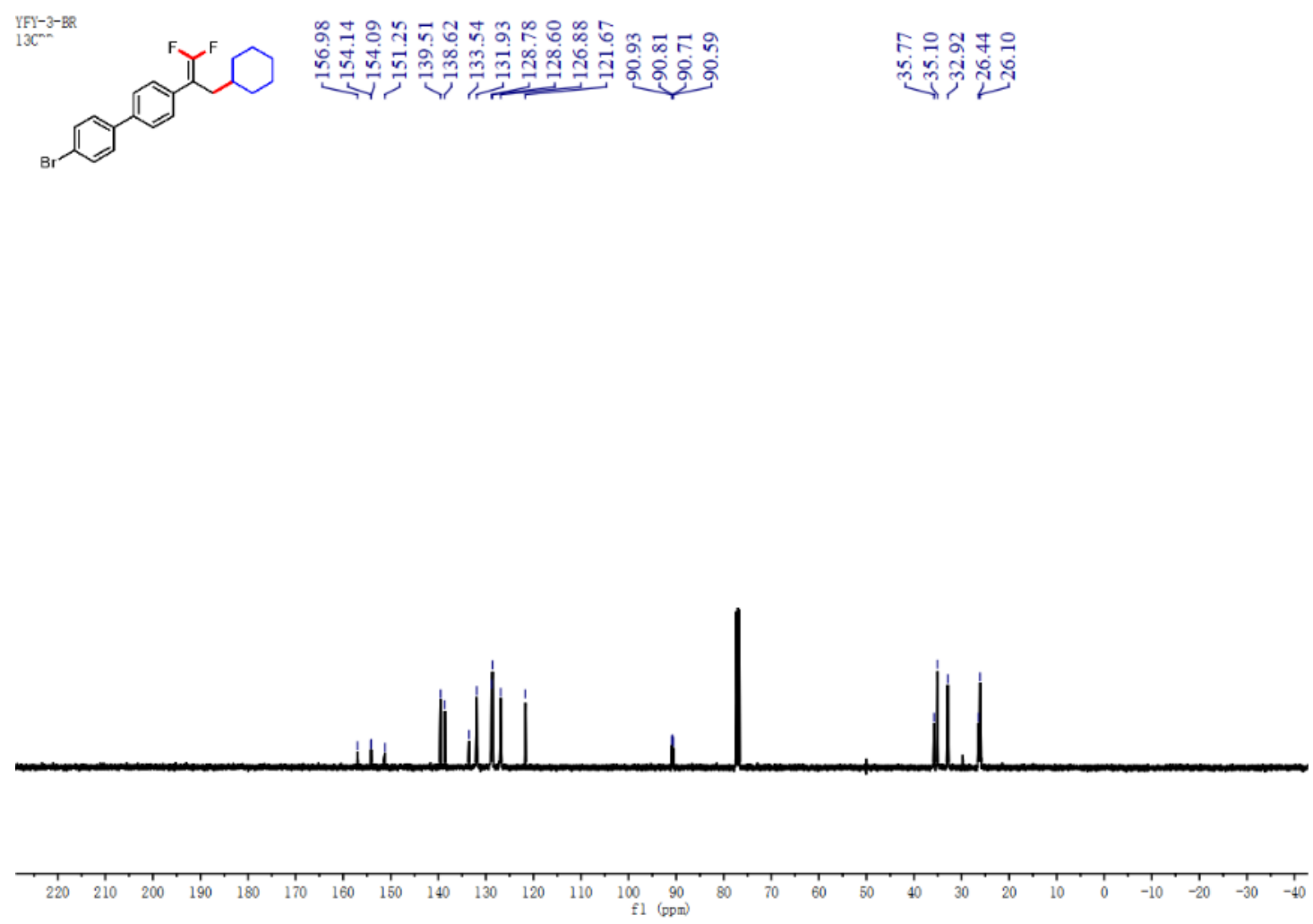

${ }^{19} \mathrm{~F}$ NMR (376 MHz, $\mathrm{CDCl}_{3}$ ) spectrum of compound 34
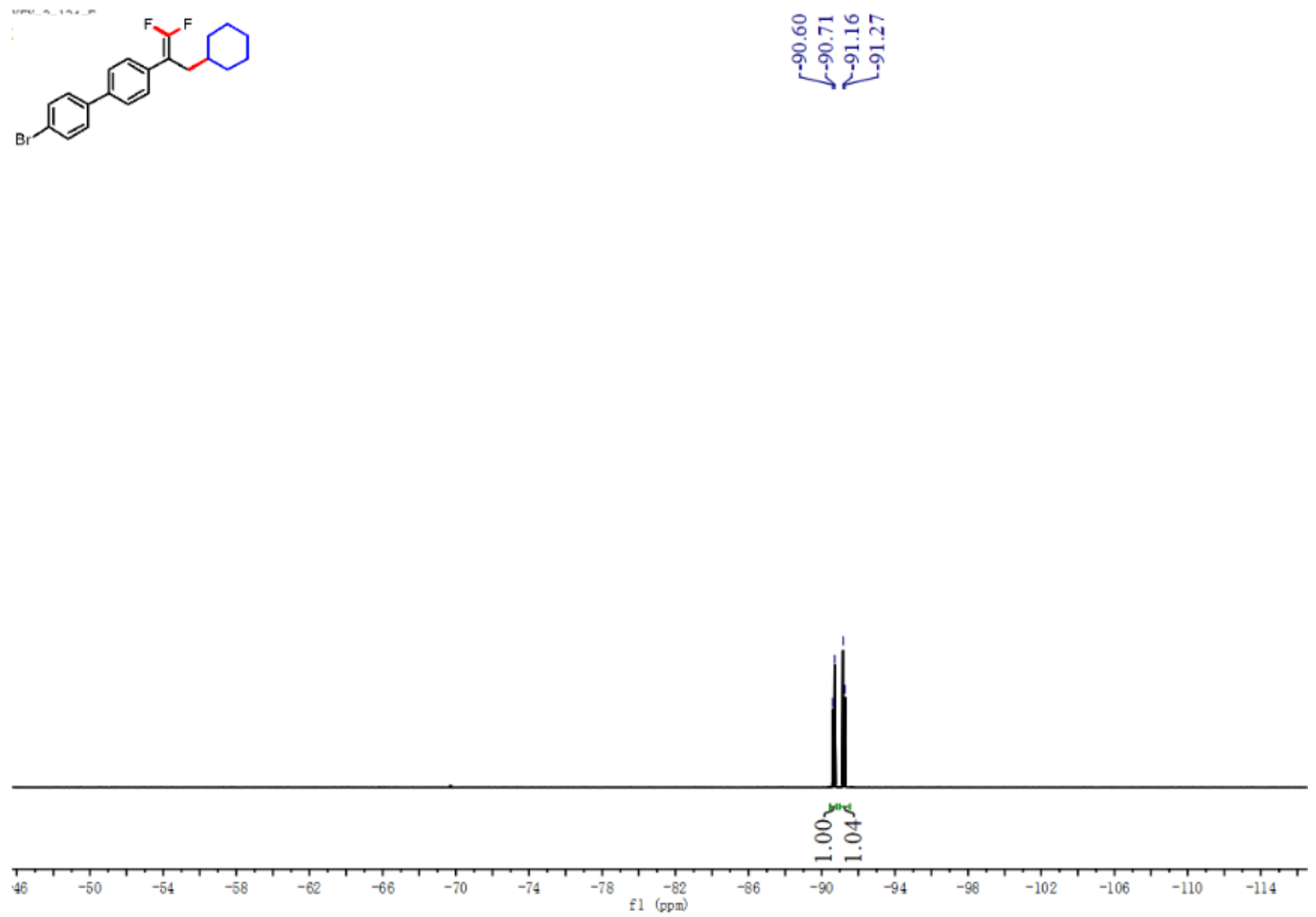

${ }^{1} \mathrm{H}$ NMR (400 MHz, $\mathrm{CDCl}_{3}$ ) spectrum of compound 35 

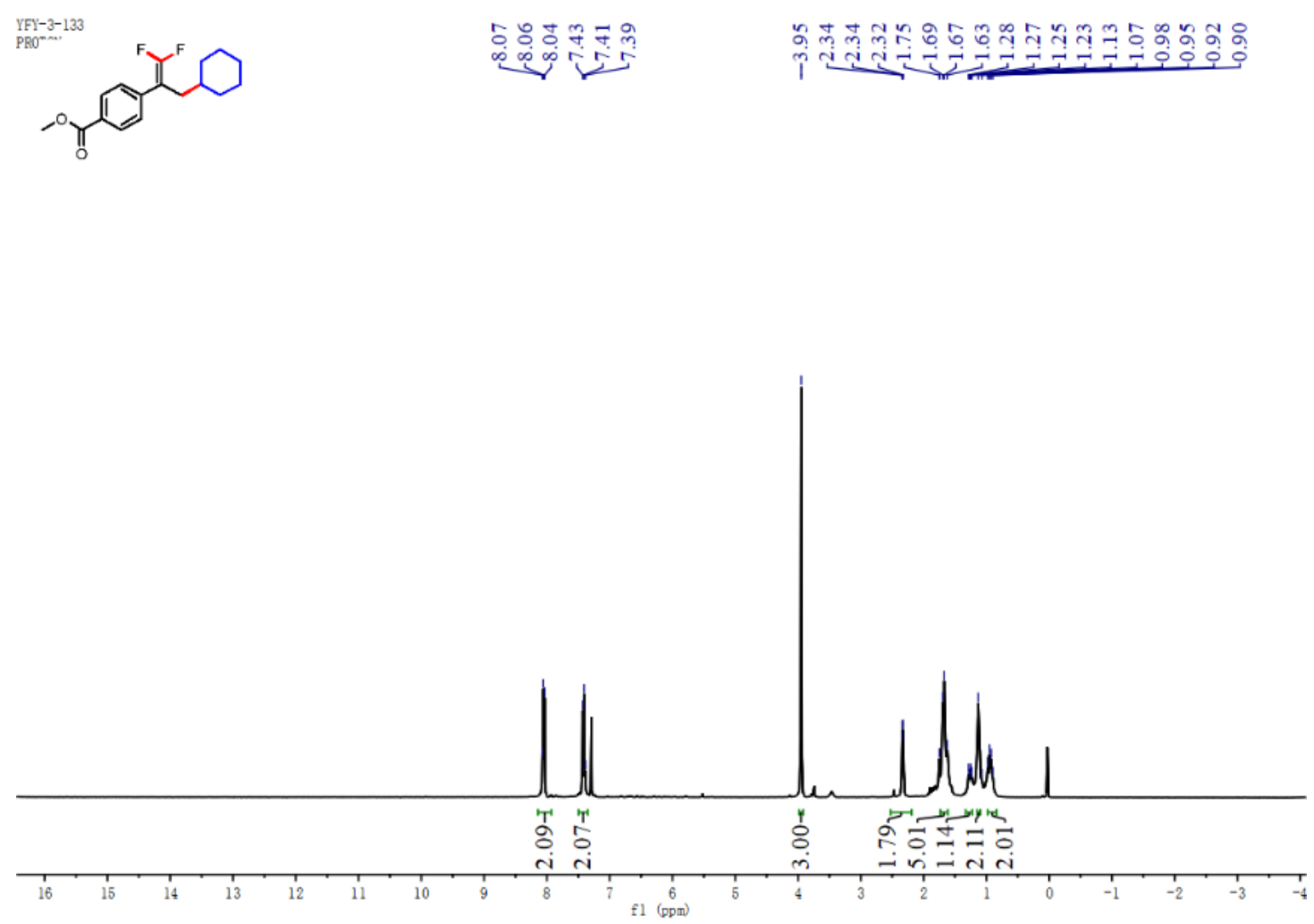

${ }^{13} \mathrm{C}$ NMR (100 MHz, $\mathrm{CDCl}_{3}$ ) spectrum of compound 35
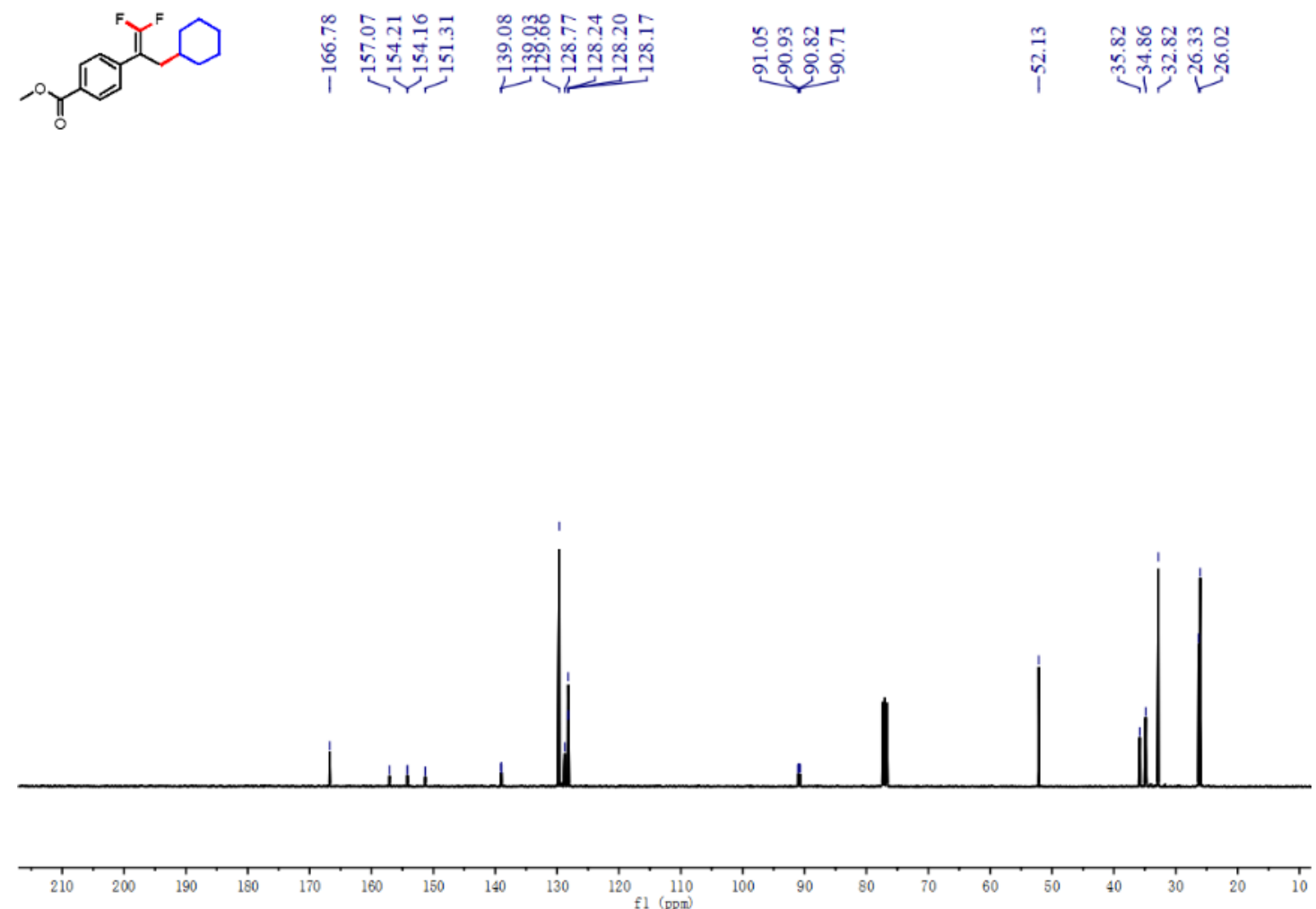

${ }^{19} \mathrm{~F} \mathrm{NMR}\left(376 \mathrm{MHz}, \mathrm{CDCl}_{3}\right.$ ) spectrum of compound 35 

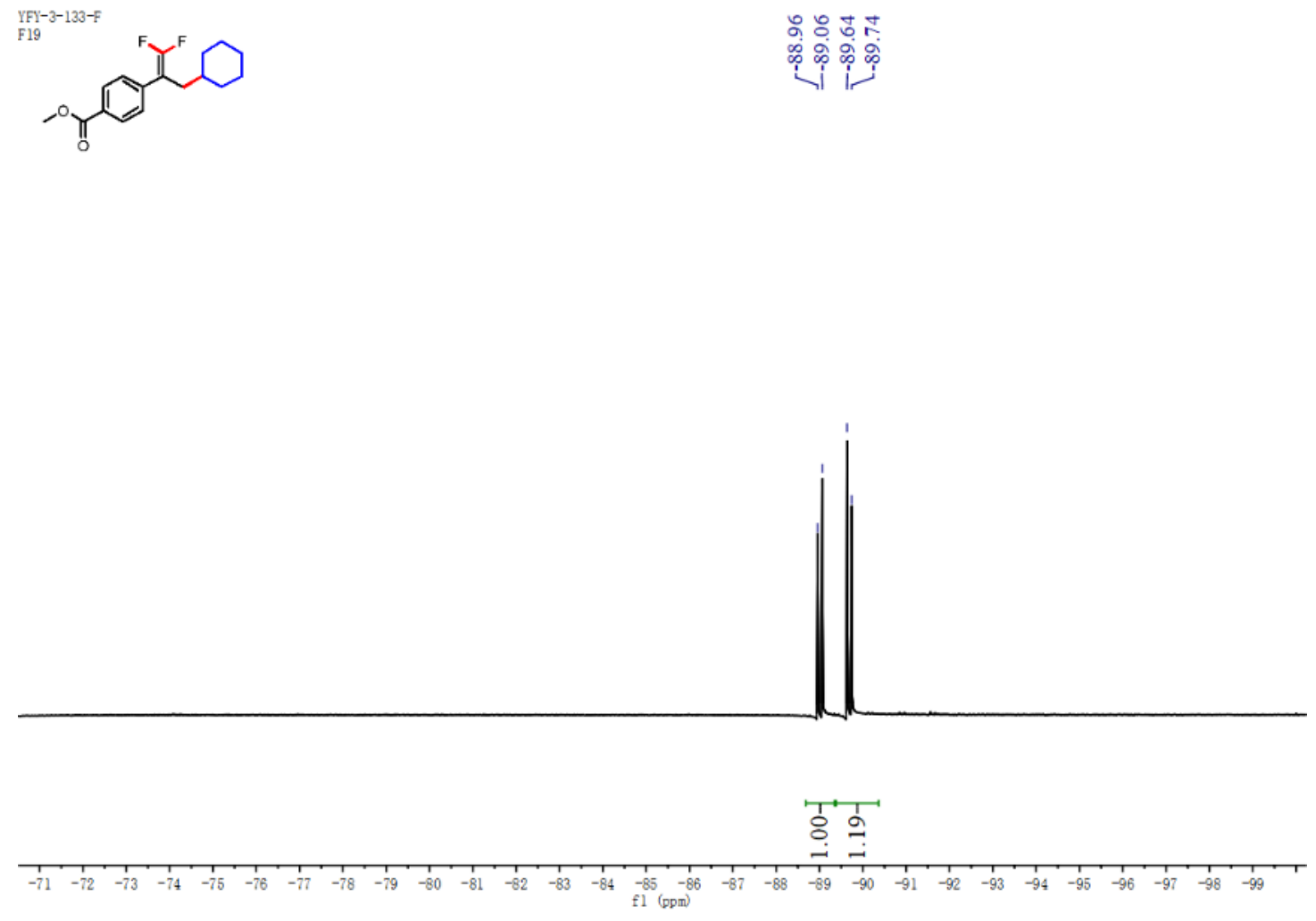

${ }^{1} \mathrm{H}$ NMR (400 MHz, $\mathrm{CDCl}_{3}$ ) spectrum of compound 36
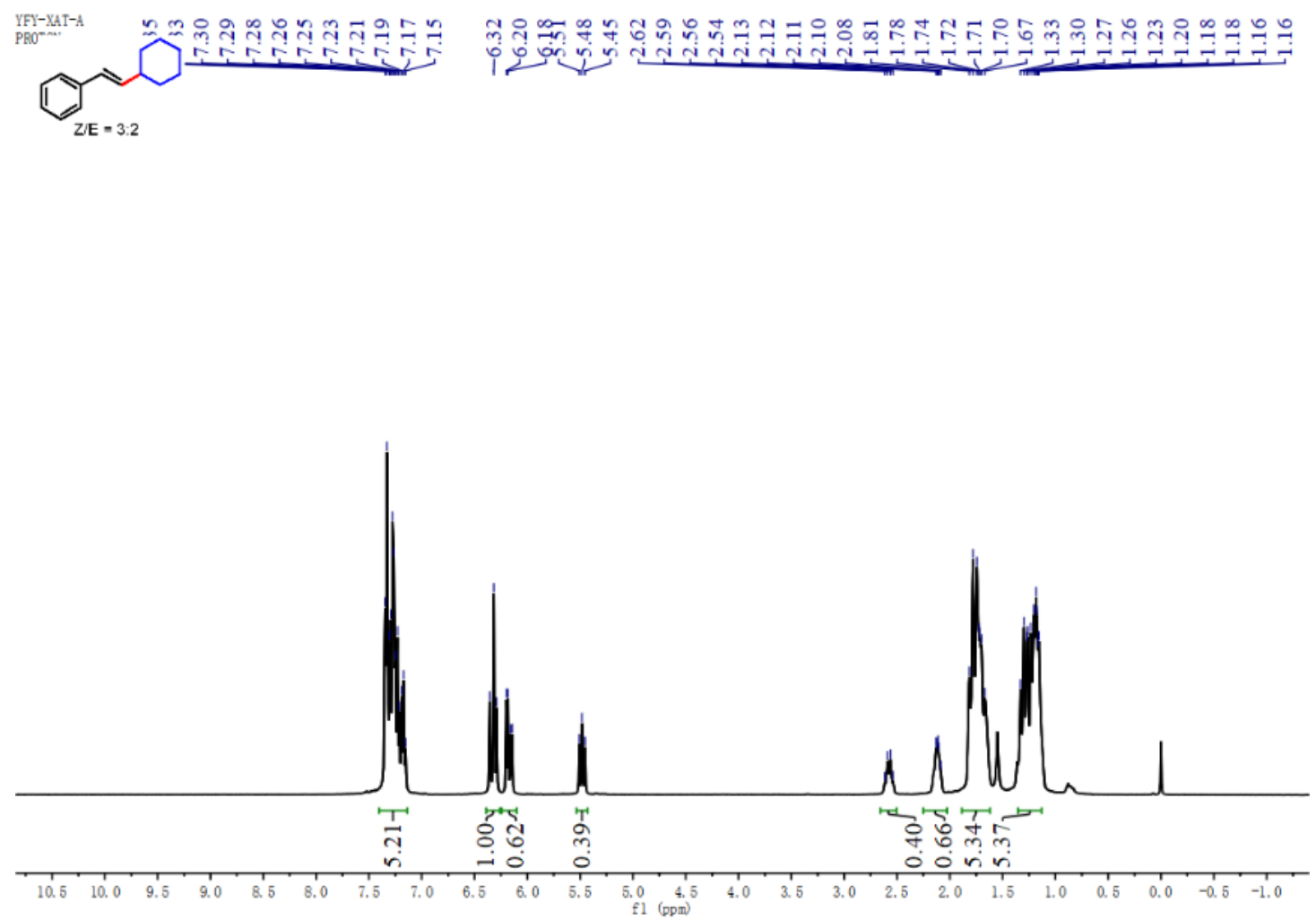

${ }^{13} \mathrm{C}$ NMR (100 MHz, $\mathrm{CDCl}_{3}$ ) spectrum of compound 36 


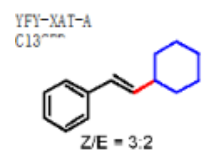

JE $=3.2$
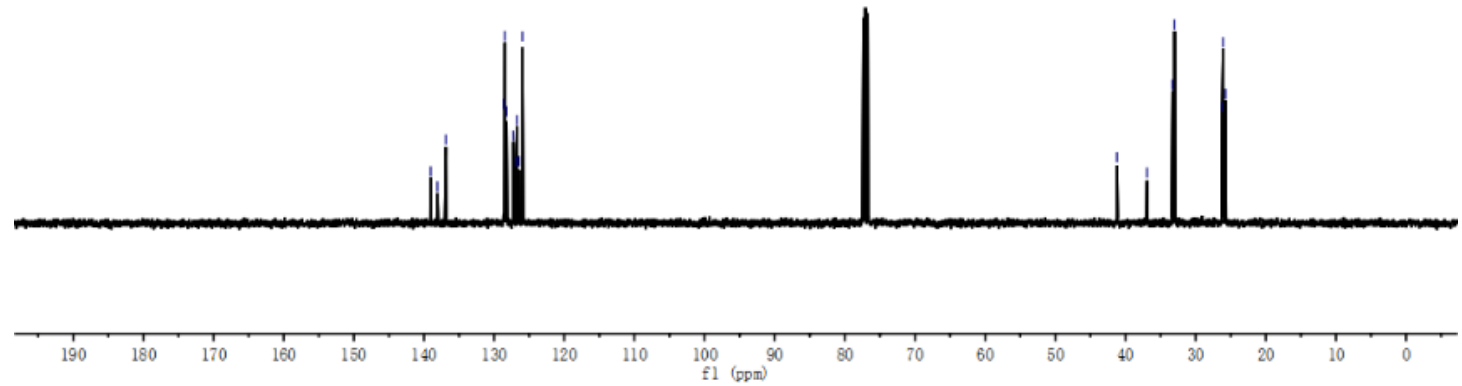

${ }^{1} \mathrm{H}$ NMR (400 MHz, $\mathrm{CDCl}_{3}$ ) spectrum of compound 37
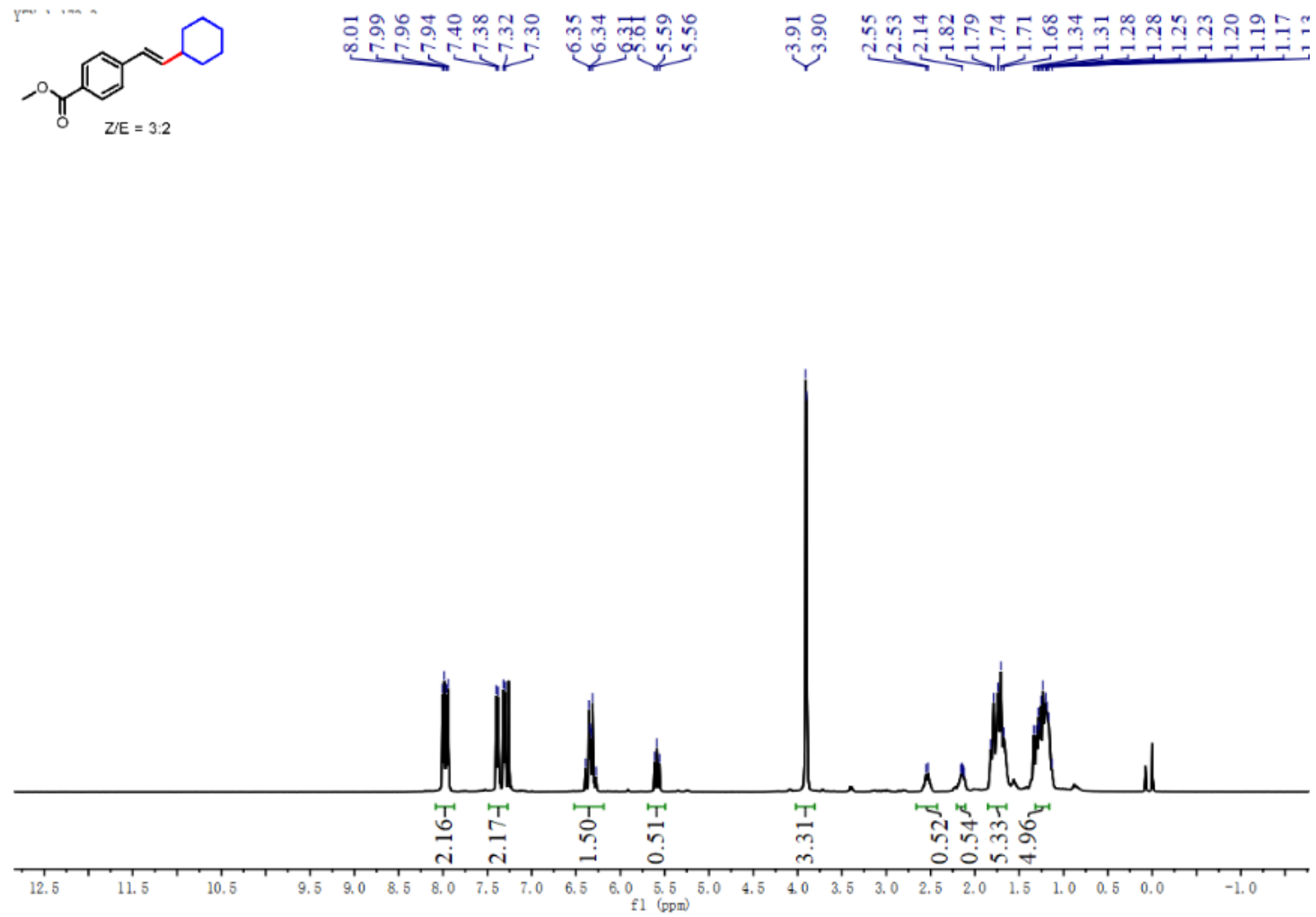

${ }^{13} \mathrm{C}$ NMR (100 MHz, $\mathrm{CDCl}_{3}$ ) spectrum of compound 37 

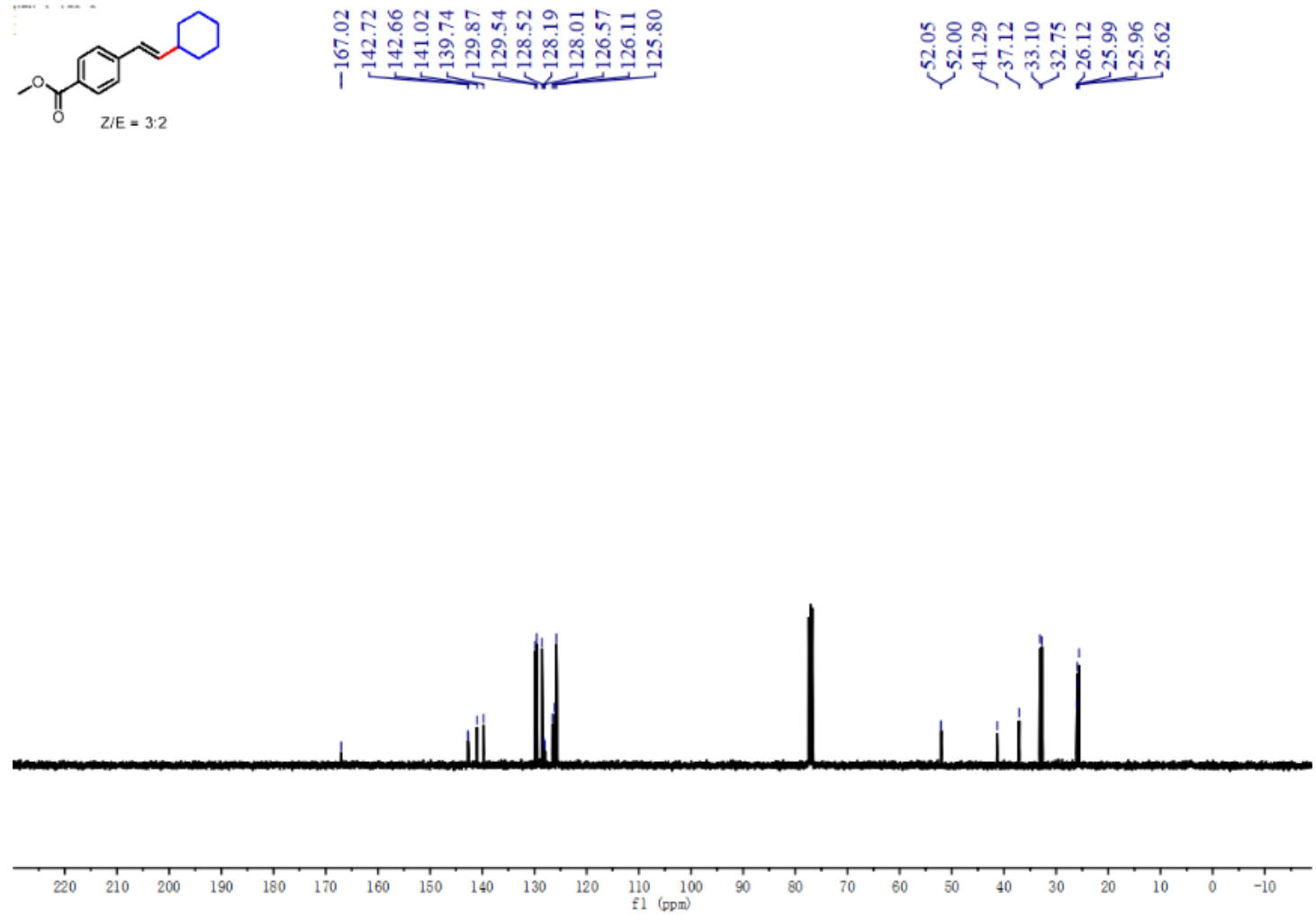

${ }^{1} \mathrm{H}$ NMR (400 MHz, $\mathrm{CDCl}_{3}$ ) spectrum of compound 38
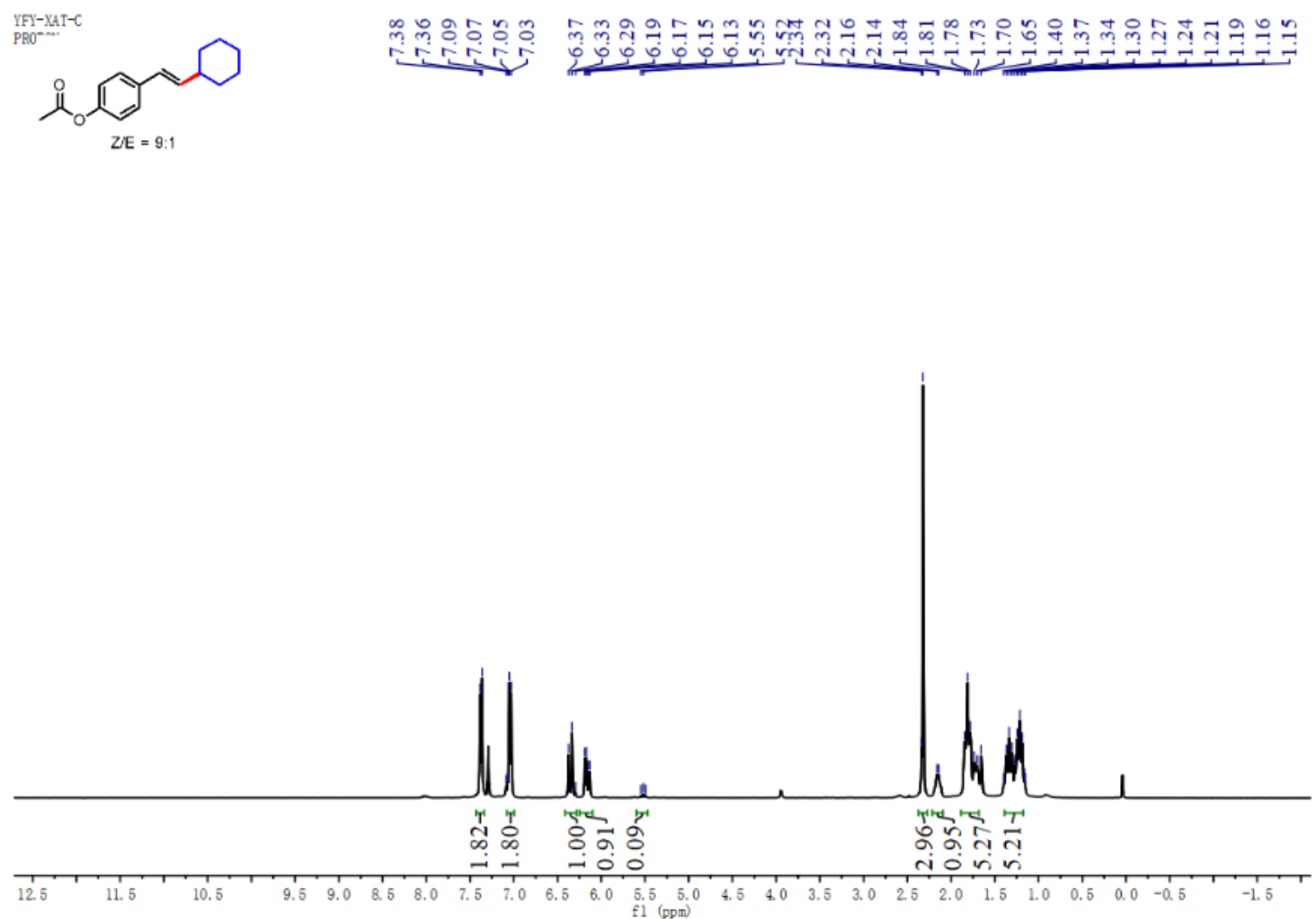

${ }^{13} \mathrm{C}$ NMR (100 MHz, $\mathrm{CDCl}_{3}$ ) spectrum of compound $\mathbf{3 8}$ 

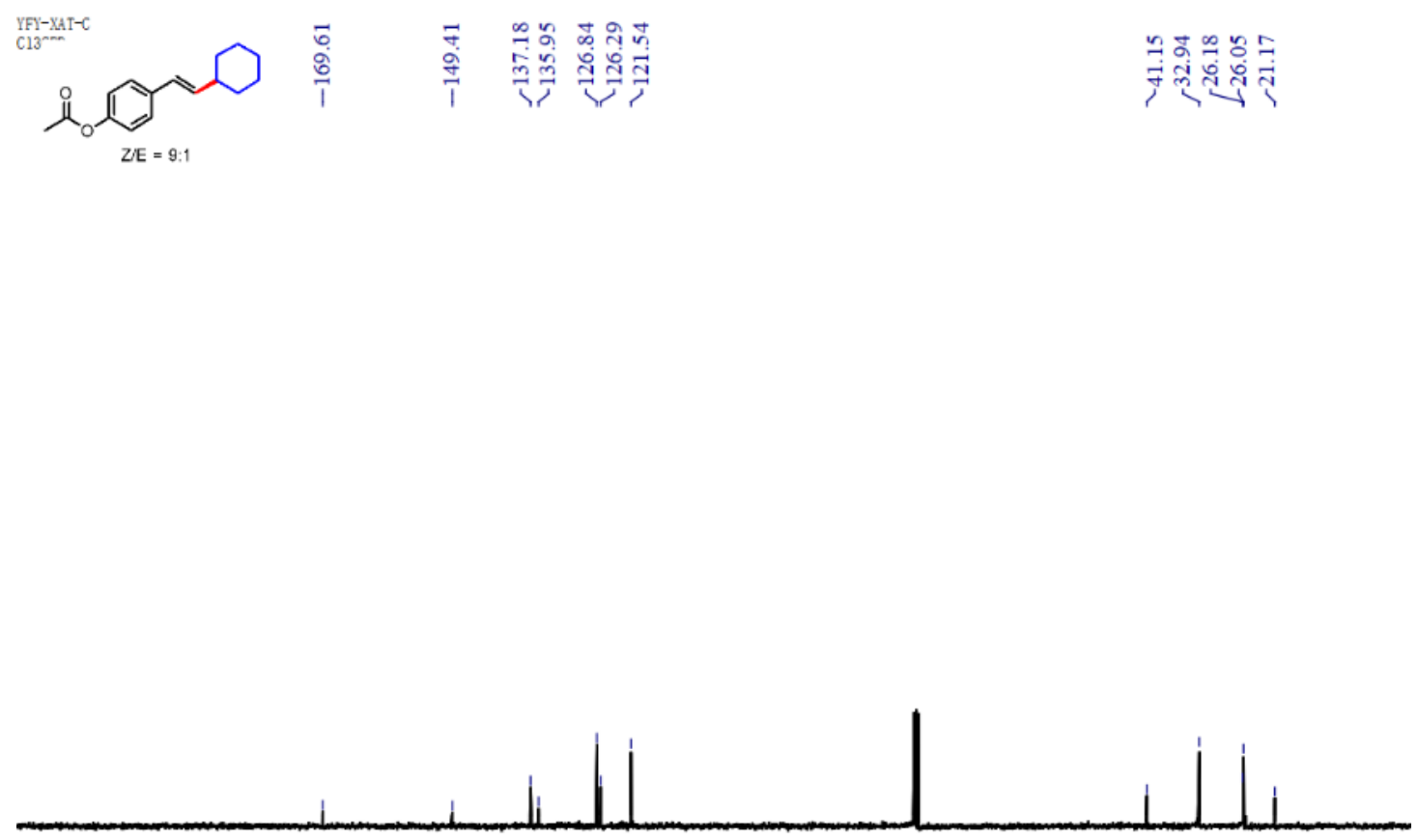

${ }^{1} \mathrm{H}$ NMR (400 MHz, $\mathrm{CDCl}_{3}$ ) spectrum of compound 39
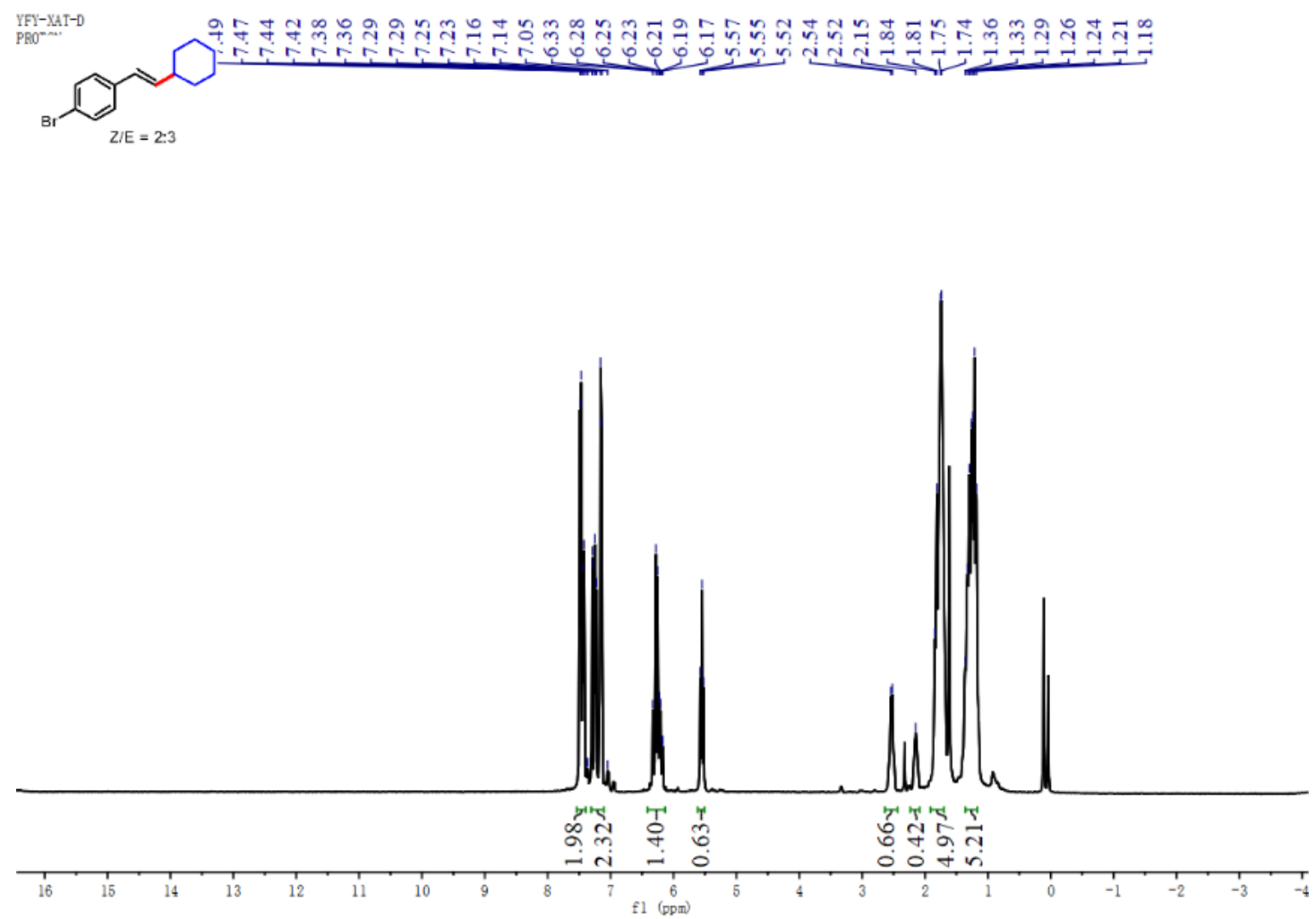

${ }^{13} \mathrm{C}$ NMR (100 MHz, $\mathrm{CDCl}_{3}$ ) spectrum of compound 39 

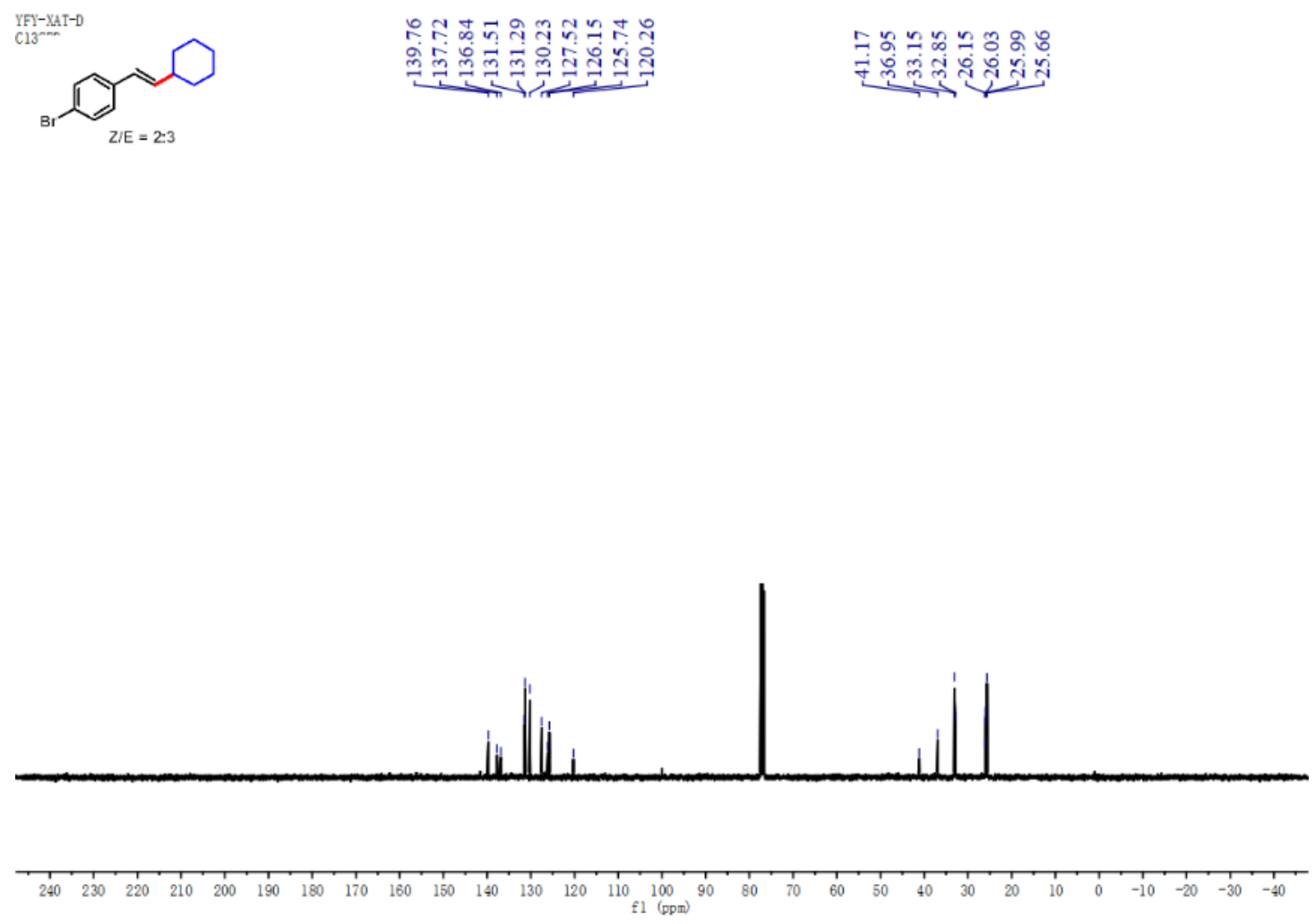

${ }^{1} \mathrm{H}$ NMR (400 MHz, $\mathrm{CDCl}_{3}$ ) spectrum of compound 41
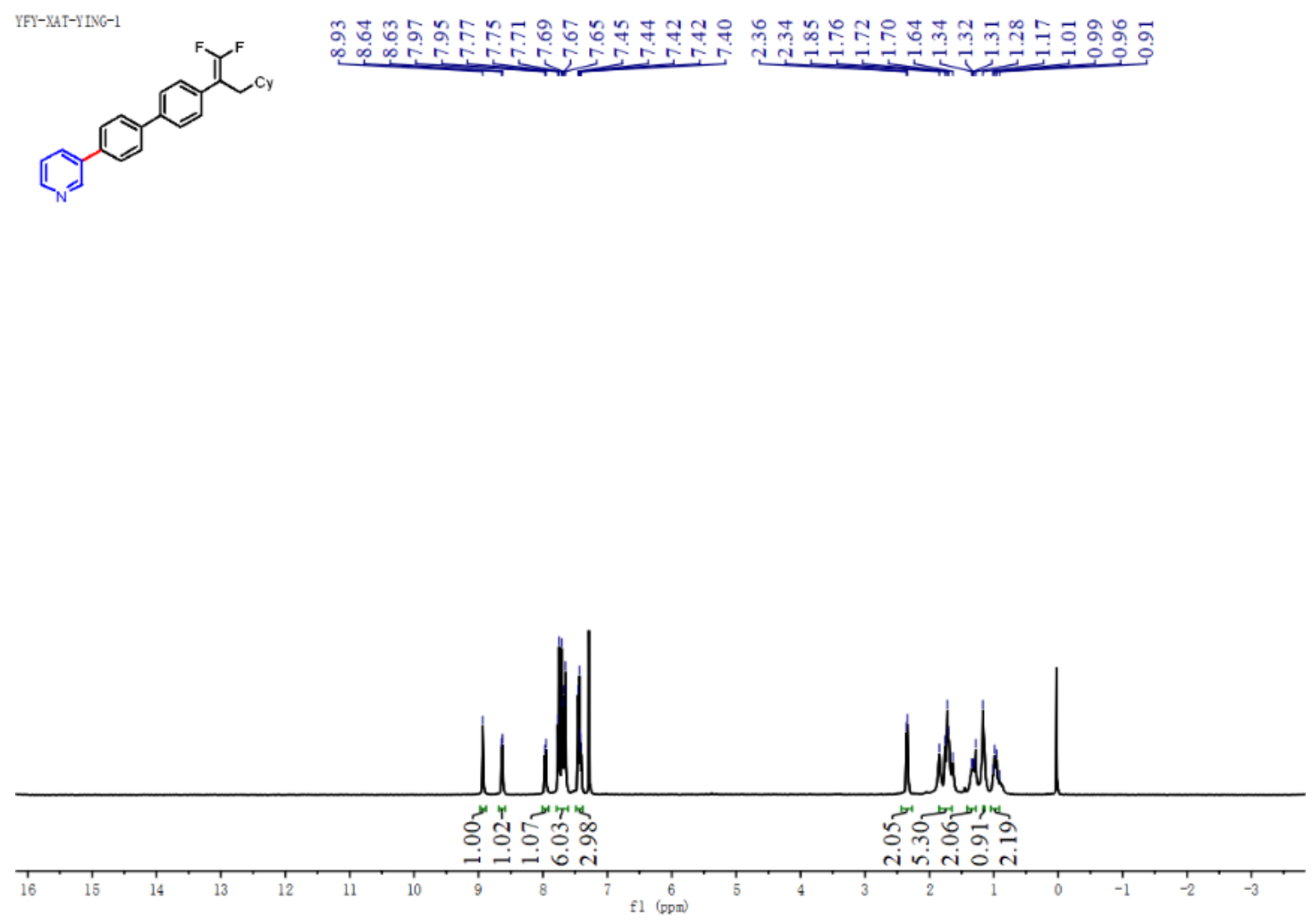

${ }^{13} \mathrm{C}$ NMR (100 MHz, $\left.\mathrm{CDCl}_{3}\right)$ spectrum of compound 41 

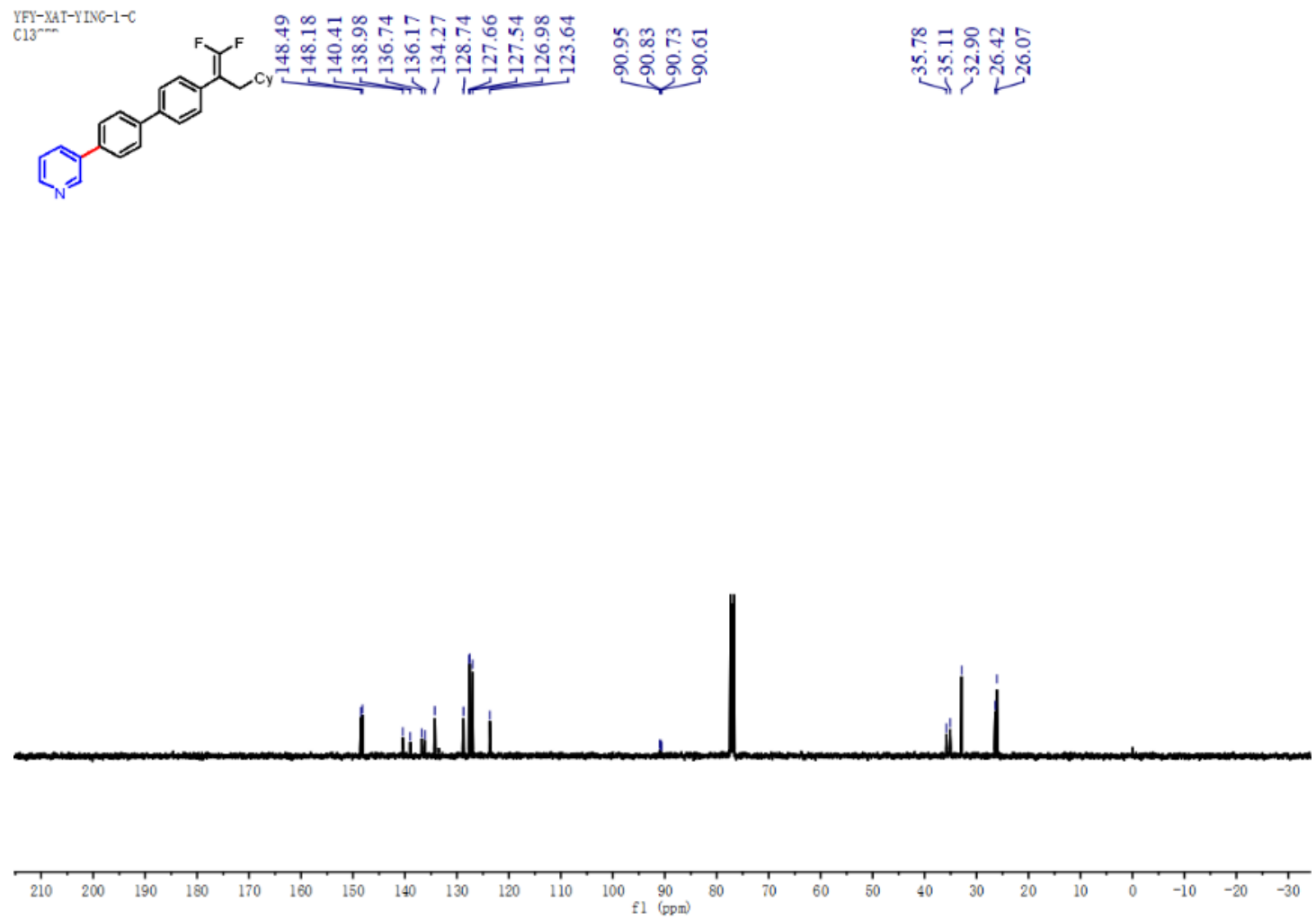

${ }^{19} \mathrm{~F}$ NMR (376 MHz, $\mathrm{CDCl}_{3}$ ) spectrum of compound 41
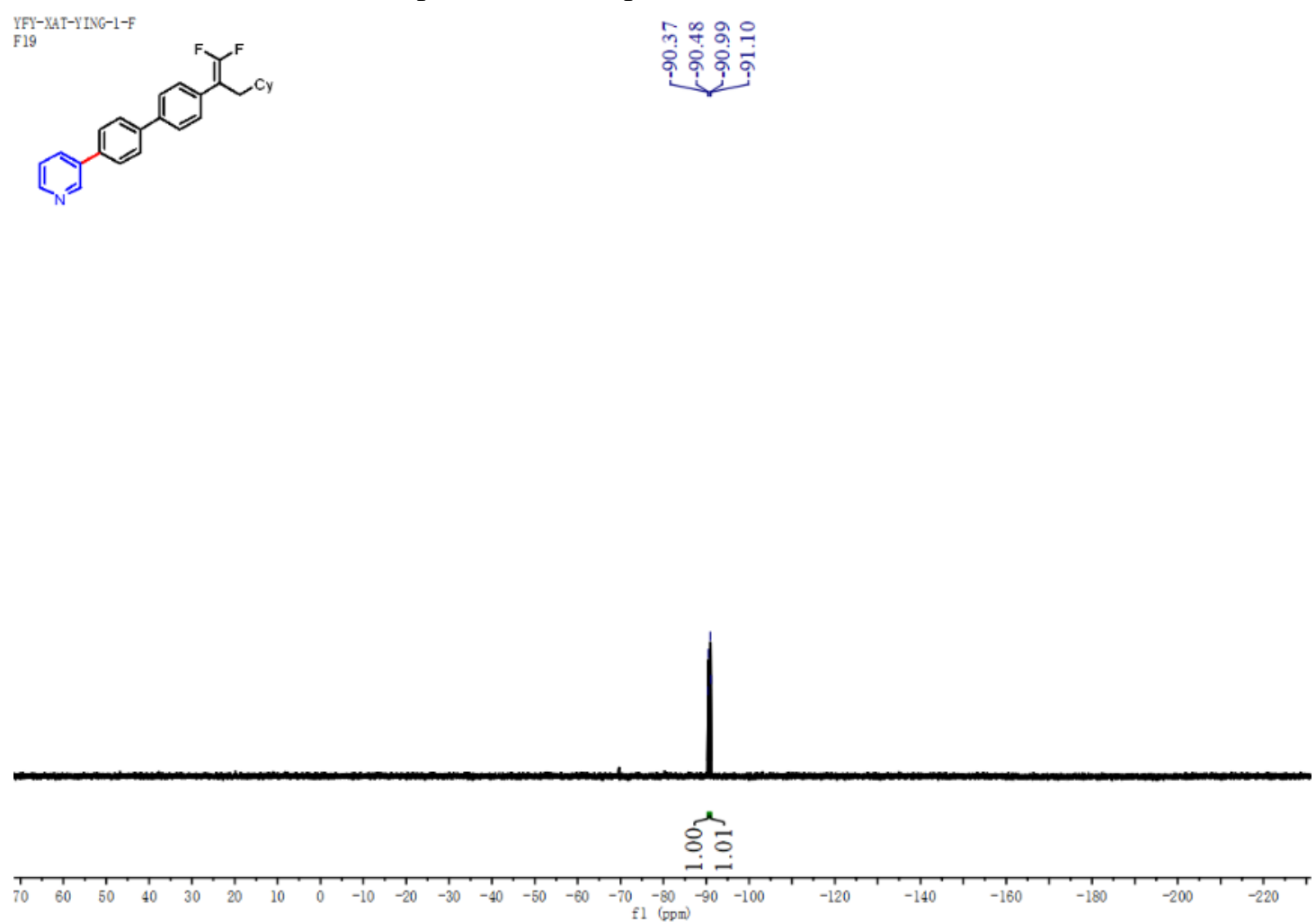